\title{
$\frac{10}{319} 19$ \\ SANDIA REPORT
}

SAND90-1344 $\cdot$ UC-253

Unlimited Release

Printed December 1990

\section{Phase I Drilling Operations at the Magma Energy Exploratory Well (LVF 51-20)}

John T. Finger, Ronald D. Jacobson

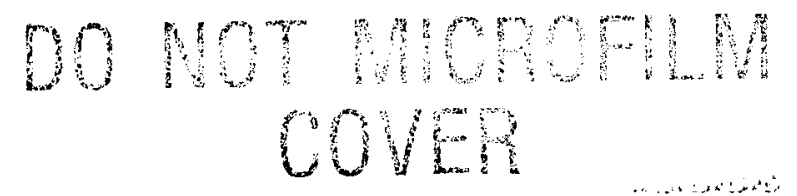

Prepared by

Sandia National Laboratories

Albuquerque, New Mexico 87185 and Livermore, California 94550

for the United States Department of Energy

under Contract DE-AC04-76DP00789

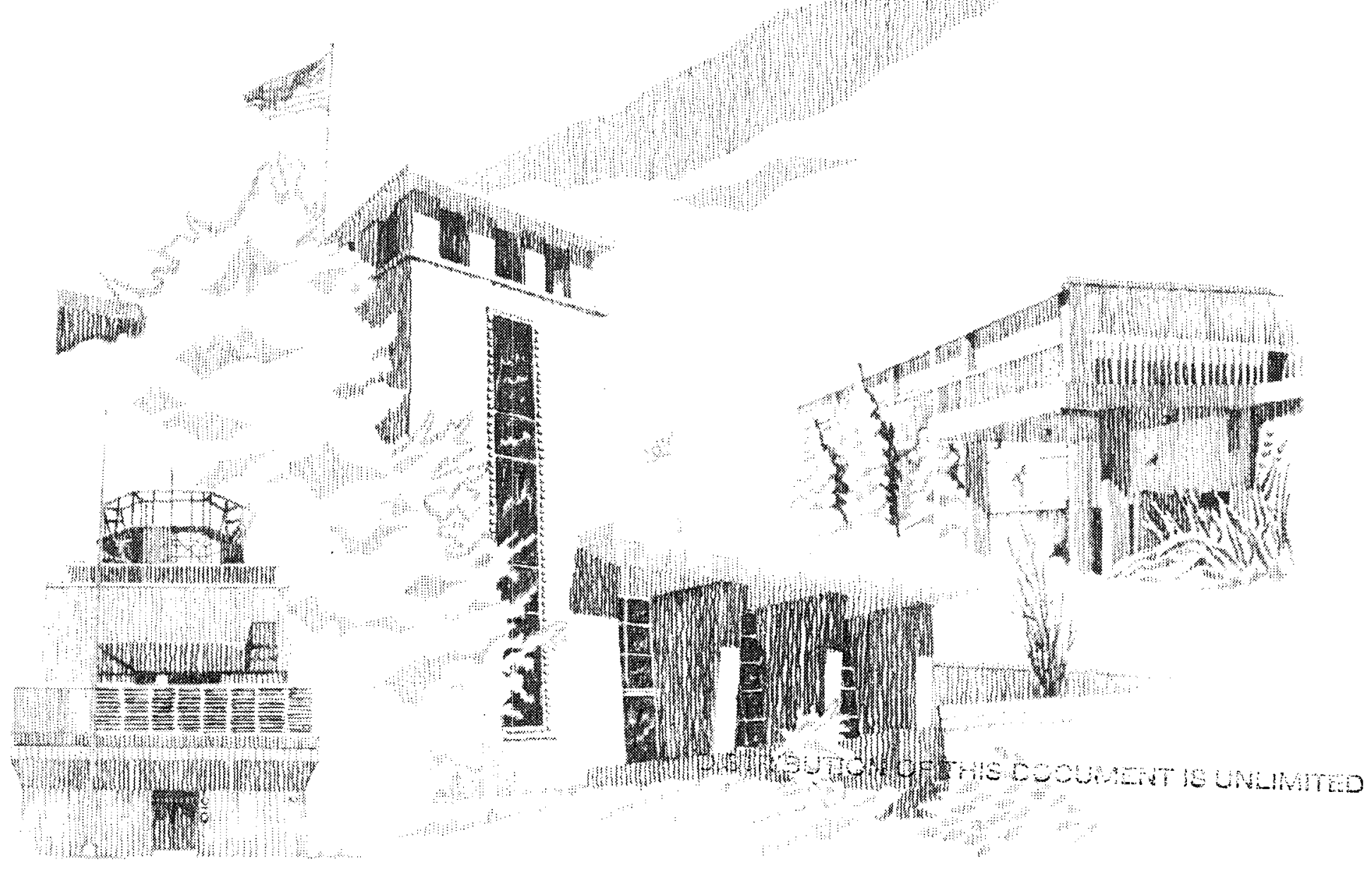


Issued by Sandia National Laboratories, operated for the United States Department of Energy by Sandia Corporation.

NOTICE: This report was prepared as an account of work sponsored by an agency of the United States Government. Neither the United States Government nor any agency thereof, nor any of their employees, nor any of their contractors, subcontractors, or their employees, makes any warranty, express or implied, or assumes any legal liability or responsibility for the accuracy, completeness, or usefulness of any information, apparatus, product, or process disclosed, or represents that its use would not infringe privately owned rights. Reference herein to any specific commercial product, process, or service by trade name, trademark, manufacturer, or otherwise, does not necessarily constitute or imply its endorsement, recommendation, or favoring by the United States Government, any agency thereof or any of their contractors or subcontractors. The views and opinions expressed herein do not necessarily state or reflect those of the United States Government, any agency thereof or any of their contractors.

Printed in the United States of America. This report has been reproduced directly from the best available copy.

Available to DOE and DOE contractors from

Office of Scientific and Technical Information

PO Box 62

Oak Ridge, TN 37831

Prices available from (615) 576-8401, FTS 626-8401

Available to the public from

National Technical Information Service

US Department of Commerce

5285 Port Royal Rd

Springfield, VA 22161

NTIS price codes

Printed copy: A07

Microfiche copy: A01 


\section{DISCLAIMER}

This report was prepared as an account of work sponsored by an agency of the United States Government. Neither the United States Government nor any agency Thereof, nor any of their employees, makes any warranty, express or implied, or assumes any legal liability or responsibility for the accuracy, completeness, or usefulness of any information, apparatus, product, or process disclosed, or represents that its use would not infringe privately owned rights. Reference herein to any specific commercial product, process, or service by trade name, trademark, manufacturer, or otherwise does not necessarily constitute or imply its endorsement, recommendation, or favoring by the United States Government or any agency thereof. The views and opinions of authors expressed herein do not necessarily state or reflect those of the United States Government or any agency thereof. 


\section{DISCLAIMER}

Portions of this document may be illegible in electronic image products. Images are produced from the best available original document. 
SAND90-1344

Unlimited Release

Printed December 1990

PHASE I DRILLING OPERATIONS

MAGMA ENERGY EXPLORATORY WELL

(LVF $51-20$ )

John T. Finger

Ronald D. Jacobson

Geothermal Research Division 6252

ABSTRACT

This report describes the Phase I drilling operations for the Magma Energy Exploratory Well near Mammoth Lakes, California. An important part of the Department of Energy's Magma Energy Program, this well is designed to reach an ultimate depth of 20,000 feet or a bottomhole temperature of $500^{\circ} \mathrm{C}$, whichever comes first. There will be four drilling phases, at least a year apart, with scientific investigations in the borehole between the drilling intervals.

Phase I of this project resulted in a 20 " cased hole to 2558 feet, with 185 feet of coring beyond that. This document comprises a narrative of the daily activities, copies of the daily mud and lithologic reports, time breakdowns of rig activities, inventories of lost circulation materials, temperature logs of the cored hole, and a strip chart mud log.

This work was supported by the U. S. Department of Energy at Sandia National Laboratories unaer contract DE-AC04-76DP00789.

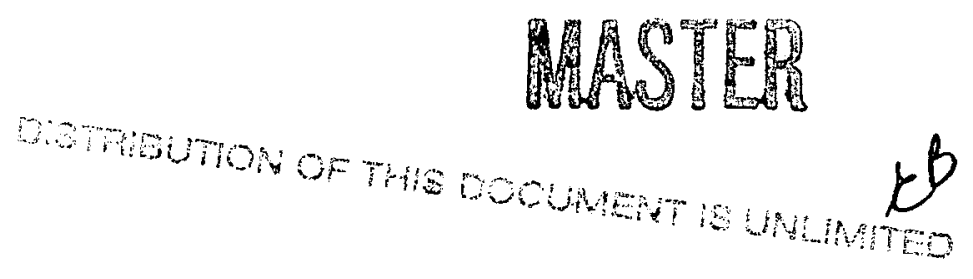


TABLE OF CONTENTS

Introduction 1

Narrative of Daily Operations 2

Appendix A - Time Breakdown for Drilling Activities 24

Appendix B - Bit Records 25

Appendix C - Specifications for Drill and Coring Rigs 26

Appendix D - Listing of Lost Circulation Materials and Drilling Fluid Additives in Phase I 27

Appendix E - Temperature Log 28

Appendix F - Directional Log 30

Appendix G - Daily Mud Logger's Reports 34 


\section{INTRODUCTION}

Molten rock, or magma, beneath the Earth's surface is an enormous energy resource. Estimates by the US Geological Survey suggest that magma less than six miles deep beneath the continental United States contains 50,000 to 500,000 quads of energy (1). For comparison, the total energy consumption in the US last year was about 80 quads.

The US Department of Energy, Geothermal Technology Division, sponsors a program managed by sandia National Laboratories to examine the feasibility of extracting this energy for commercial power production. An earlier study by sandia showed that tapping this magma energy is technically possible, but development engineering to make it a reality will be extremely challenging. This well will allow better evaluation of the continental magma resource and development of a magma energy extraction system.

We are drilling at this location because extensive geophysical evidence indicates the existence of a magma body at a depth of six to seven kilometers (2). Much of the evidence comprises seismic data, but an especially compelling observation is that the drill site has risen two feet in the last ten years. Scientists infer that this rise was caused by fresh magma injected into the underlying chamber. This location, in fact, was originally prepared by a commercial geothermal company for an exploratory well. Their drilling plans did not materialize, and they agreed to let sandia use the site.

The magma energy concept requires the ability to predict the location of large quantities of shallow magma. Volcanologists believe, and geophysical measurements suggest, that regions like Long valley contain these magma bodies, but their existence has not been directly verified by drilling. Resolving the question of a magma body's presence here will be the most important result of this exploratory well.

The ultimate goal of the Magma Energy Program is to drill into magma and insert a heat exchanger for long-term experiments. Although researchers from sandia successfully used a small drill rig to penetrate and perform tests in a still-liquid lava lake in Hawaii (3), no long-term, large-scale experiment in molten rock has ever been done. The Magma Energy Exploratory Well is a precursor to an energy extraction experiment, and we believe that the experience of drilling in this unique environment that approaches, but does not contact, the magma chamber will be invaluable.

1. Muffler, L.J.P., (Editor), "Assessment of Geothermal Resources of the United States", US Geological Survey Circular 790, 1979

2. Rundle, J.B., and D.P. Hill, "The geophysics of a restless caldera - Long Valley", Ann. Rev. Earth Planet. Sci., 16, 1988 3. Hardee, H.C., J.C. Dunn, R.G. Hills, and R.W. Ward, "Probing the melt zone of Kilauea Iki Lava Lake, Kilauea Volcano, Hawaii", Geophysical Review Letters, 8 no. 12, 1211-1214 
NARRATIVE OF DRILLING OPERATIONS

The first of the four planned drilling phases, with a designed target depth of 2500 feet (see Figure 1) was completed in October 1989. The following narrative gives a day-by-day summary of the drilling and coring activities, and the problems encountered.

[A list of abbreviations used in this narrative is on page 23.]

$8 / 1$

Spud continues to approach, but slowly. Loffland is picking up the bottom hole assembly; mud is mixed; most welding is done.

2200 - Loffland ready to spud, but bit breaker does not fit their rotary table (it's for a $271 / 2$ " table, theirs is $371 / 2^{\prime \prime}$ ).

$8 / 2$

Temporary fix for the bit breaker problem is to weld pins on the bottom that will fit the rotary. New breaker will be fabricated in Bakersfield today.

0630 - Spudded, made $12 \mathrm{ft}$

0700 - Lost circulation. Mixing a 50 bbl LCM pill.

0930 - Some problems with rig equipment: there is a hole in one of the shale shakers, leaking mud; also down with one of the mud pumps. on maintenance time at 0930, off at 1300 .

1300 - Lost circulation is extreme, have lost over 1000 bbl mud, including LCM. Drilling to about $90^{\circ}$ (bottom of $40^{\prime \prime}$ mud riser is at 64' KB) with 26" bit and 36" hole opener - no returns; will set 125 sack (202 $\mathrm{ft}^{3}$ ) cement plug. [125 sacks class G cement, $40 \%$ silica flour, $3 \% \mathrm{CaCl}]$

1830 - Cement in place - plug \#1 - at $1830 \mathrm{hrs,} \mathrm{doesn't} \mathrm{look} \mathrm{good}$ because only about $3^{\prime}$ at the bottom of the rods shows cement on the outside.

2130 - Tag bottom, and find that plug extends about 2' up.

2400 - Mix and set another $202 \mathrm{ft}^{3} \mathrm{plug}-\# 2$.

$8 / 3$

0600 - Second cement plug has gone away completely; mixing another 125 sacks cement with 2:1 perlite for a lighter weight, higher yield mixture. Running Flo-Chek and calcium chloride water ahead of cement to gel up the formation.

0730 - Cement in place. Pumped plug in two equal parts -plugs \#3 ana \#4, total volume $310 \mathrm{ft}^{3}$. WOC 
DEPT и

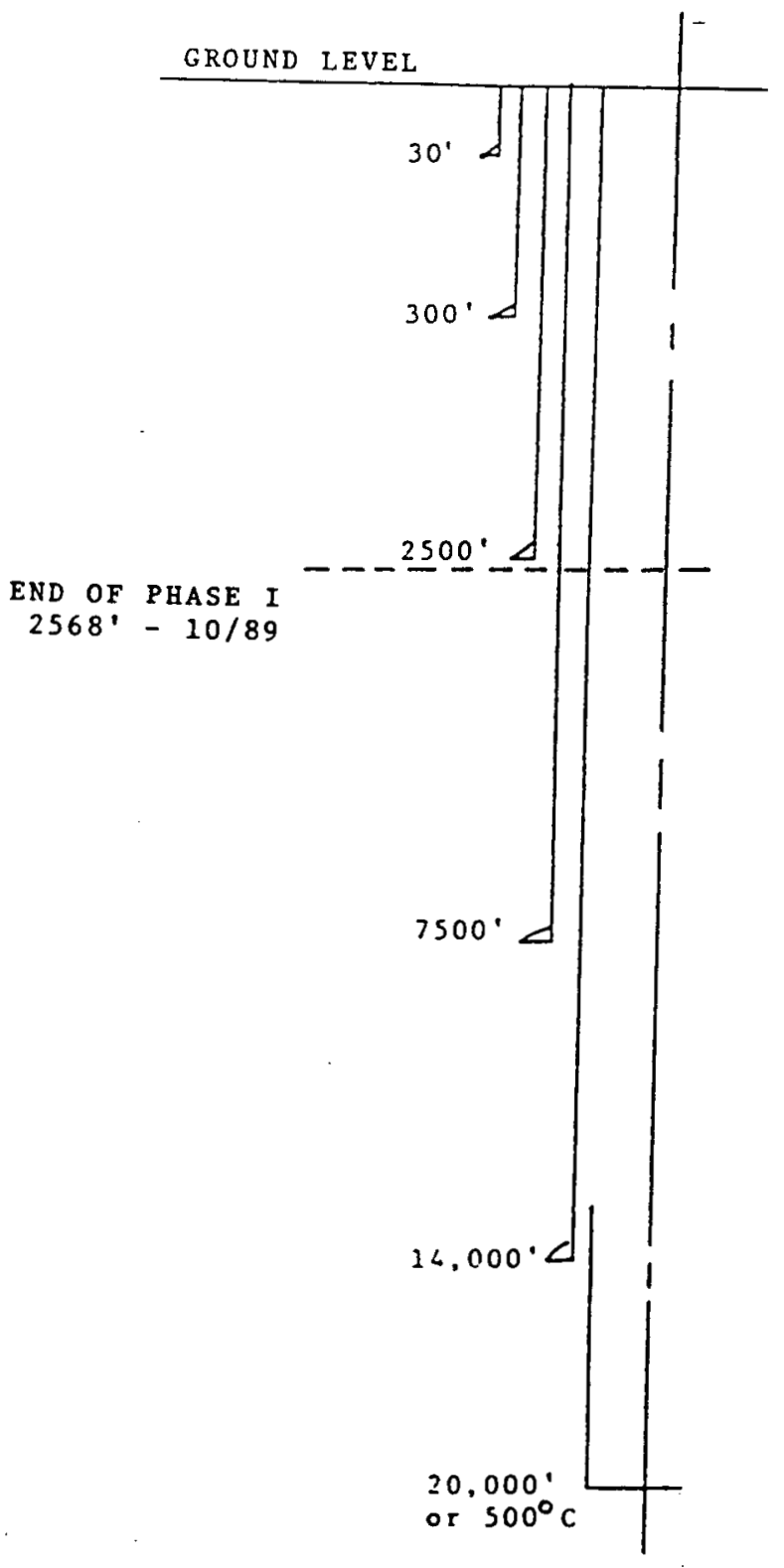

$48^{\prime \prime} \quad 40^{\prime \prime}$

36

$30 "$ .625 wall

$28.75^{\prime \prime}$

$26^{\prime \prime}$

$20 "$

$133 \pi$

$18.73^{\prime \prime}$

$17-1 / 2^{\circ}$

13-1/2"

$12.37 "$

81.40

12-1/4" $\begin{array}{r}9-5 / 80 \\ 53.50\end{array}$

$12-1 / 4^{\circ} \quad 5-5 / 8^{\prime \prime}$
$53.50^{\circ}$

$12-1 / 4^{\circ} \quad 9-5 / 80$
53.50

$6.54^{\circ}$

6.

8-1/2" OPEN HOLE.

Figure 1 -- Diagram of well design, showing hole and casing sizes 
1130 - Threw 10 sacks of bentonite, 6 bags of walnut shells, and approximately 450 empty mud and cement sacks in the hole. Regained circulation and pumped appr $400 \mathrm{bbl}$ water through the hole to clean it up before circulating mud again.

1400 - Laid down the 36" hole opener and will drill ahead with the 26" bit; some concern that the h.o. might be causing deviation in the wellbore.

1730 - Drilled ahead to 96'. Partially lost circulation again (30$40 \%$ returns) and decided to set another plug. Spotted at the bottom of the 40" mud riser: $10 \mathrm{bbl}$ calcium chloride, $10 \mathrm{bbl}$ sodium silicate (Flo-Chek), and approx $310 \mathrm{ft}^{3}$ of 2:1 perlite/cement - \#5.

1930 - Cement in place. Woc

\section{$8 / 4$}

0530 - Tagged top of cement at 57' (inside the $40^{\prime \prime}$ ) and found it still soft; will wait another couple of hours and drill it. Picked up 36" h.o. to center punch the hole.

0830 - Began drilling with good returns, but after getting through the cement into fresh rock started losing about $10-20 \mathrm{bbl}$ per minute. Drilled ahead to $98^{\prime}$ and decided to stop for another cement job

1200 - Pumped another plug - \#6 - with $10 \mathrm{bbl}$ calcium chloride ahead of $300 \mathrm{ft}^{3} 2: 1$ perlite/cement. Will woC until approximately 1900.

1915 - Tagged cement at $67^{\prime}$, cleaned out cement to $78^{\prime}$ with full returns, but the cement cuttings started appearing green, so will woc until midnight.

\section{$8 / 5$}

0003 - started rotating at $71^{\prime}$ and got good cement with full returns.

0230 - Drilled kelly down with the 36" h.0. (96'), laid down the h.o. and picked up the 26" BHA. Drilling ahead with 26", holding back on WOB to limit ROP to about $40 \mathrm{ft} / \mathrm{hr}$.

0730 - Depth is $143^{\prime} ;$ drilling is OK, although losing about 400 $\mathrm{bbl} / \mathrm{hr}$ mud. There is some problem with the shale shakers, they will not take the full flow of 1000 gpm without overflowing.

1030 - Survey at $167^{\prime}$; inclination $=34^{\circ}$.

1300 - Survey at $230^{\prime} ;$ inclination $=1 / 4^{\circ}$.

1730 - Drilled ahead with $26^{\prime \prime}$ bit to $318^{\prime}$, where returns were lost completely. Will set another plug, $310 \mathrm{ft}^{3}$ cement/perlite, as soon 
as Halliburton can get here and driller can lay down BHA and run pipe back in hole for pumping cement.

2130 - Completed cement job - \#7 - spotted at bottom. will woc about 8 hours.

\section{$8 / 6$}

0430 - Tagged top of cement at $264^{\prime}$, but plug will not hold fluid. Pump 80 bbl LCM pill (6 sacks ea nut plug, Mud Seal, and cedar fiber); ineffective. Will set another plug.

0800 - Finished pumping second $310 \mathrm{ft}^{3} \mathrm{plug}$ - \#8. It brought the fluid level back to the flow line and the level seems to be holding. Will WOC until 1400, then tag the top of the second plug and probably begin drilling.

1400 - Begin drilling with the 36" hole opener at $94^{\prime}$ (previous depth of 36" hole). Progress is pretty good, ROP is about 30 feet per hour, with little fluid loss.

1800 - Depth 190' with 36" hole.

2300 - Reached 291' with 36" hole; we will set casing at this point because of the lost circulation problem at $318^{\prime}$. This means that the 30" casing string will be only 7 joints instead of 8 , ie, 280' instead of $320^{\prime}$. Circulating the hole clean, will run LCM pills as the pipe is coming out of the hole, rigged to run casing. Crane operator is on site to lift casing from the racks to the V-door slide.

\section{$8 / 7$}

0230 - Began running 30" casing, using the Dril-Quip stab-in connectors. Casing goes together easily, without the danger of cross-threading. Each joint takes about 25-30 minutes to run. 0530 - After third joint is added, string will not go down any

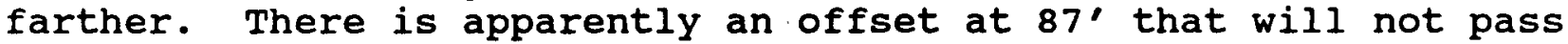
the casing string. Begin calling tool companies to locate $36^{\prime \prime}$ reamers, stabilizers, or hole openers to make a stiffer drill string that we can use to ream the hole.

2030 - Receive 36" stabilizer from Bakersfield and 36" hole opener from Nevada Test Site. Hole opener appears to be very rugged and in good condition. Begin picking up both h.o.'s to run them piggyback.

2230 - Begin running in hole with BHA = $171 / 2$ " bit (already mounted on NTS h.0./36" h.0./36" h.0./26" reamer (for stiffness)/ll" drill collar.

2400 - Reaming ahead at approximately $89^{\prime}$ depth. Drilling is very rough from $8^{\prime}$ to $8^{\prime}$, indicating more irregularities in the wellbore. 


\section{$8 / 8$}

0000-0430 - Reamed 80'-116' interval multiple times to insure smoothness.

0530 - Added another collar and drilled ahead to 113', where approximately $300 \mathrm{bbl}$ circulation loss occurred. Mixed and spotted a $50 \mathrm{bbl}$ LCM pill. Hole would still not hold fluid, so rigged for cementing.

0930 - Another $310 \mathrm{ft}^{3}$ cement/perlite plug - \#9, will woc until 1530 .

1600 - Filled hole with mud to make sure that plug was holding pressure; it did. Will WOC another 2 hours and start to ream.

1730 - Tag cement at 97'. Reaming ahead at 110'.

2400 - Reaming at 270'. Have reamed each joint down twice and lowered once without rotation

\section{$8 / 9$}

0030 - Reamed to bottom at 285'. Began to lay down tools and rig to run casing.

0530 - Began going in hole with first joint of $30^{\prime \prime}$ casing.

0700 - Fifth joint hangs up, rig cables for pull-down and torque; pipe goes in.

0840 - Casing in hole, stab mandrel in float shoe, fill casing with mud for hold-down weight, rig for casing hold-down (buoyancy force) cables, and rig for cementing.

1630 - Cement to surface, wait 5 minutes to see if cement level falls back (it doesn't), and pump 5 more minutes (total of approx $1135 \mathrm{ft}^{3}$ Class $G$ cement with $40 \%$ silica flour $+310 \mathrm{ft}^{3} 2: 1$ perlite). Drop plug and displace cement in drill pipe. Disengage drill pipe from float shoe and put about $8^{\prime}$ of cement in the bottom of the casing. Will WOC approximately 24 hours.

\section{$8 / 10$}

0600 - Tagged top of cement in casing annulus and found it at $62^{\prime}$ $\mathrm{KB} ;$ this was apparently a ring or the high side of a sloped surface, because the volume required to fill the annulus from $62^{\prime}$ is $110 \mathrm{ft}^{3}$, and $272 \mathrm{ft}^{3}$ were actually required. This implies that the top of the original cement level was below the $40 "$ shoe.

0900 - Top job completed.

1500 - Begin cutting off the 40" riser and the 30" casing to install the 30" diverter. 
2100 - Casing and riser cut and removed.

2200 - Weld 30" wellhead and begin nippling up.

$8 / 11$

0700 - Diverter and flow line installed

1200 - Began trying to test the Hydril diverter, but it would not hold pressure. Displaced the mud in the diverter with water and retested at a slightly higher pressure. Test was successful, possibly because higher pressure helped diverter to seal. Test witnessed by BLM representative Frank Dalton.

2000 - Hooked up flow and kill lines, displaced water with mud, and began drilling cement.

\section{$8 / 12$}

0230 - Directional survey at $28^{\prime} ;$ angle was $1 / 4^{\circ}$.

0500 - Lost circulation at $355^{\prime}$. Drilled from 355' to $387^{\prime}$ with partial returns; lost about 270 bbl drilling fluid.

1030 - Survey at $353^{\prime} ;$ angle was $1 / 2^{\circ}$.

1200 - $310 \mathrm{ft}^{3}$ cement plug - \#10 - at 366'.

1830 - WOC and tagged top of cement at 317'; will wOC another 4 hours.

$8 / 13$

0030 - Began drilling out cement at $317^{\prime}$.

0230 - Severe loss at $360^{\prime}$, apparently the same zone as before.

Tried circulating, no returns

0430 - Re-cemented this zone with $300 \mathrm{ft}^{3}$ cement plug - \#11; will woc until 1230.

1300 - Begin drilling out cement at 293'.

1800 - Drilling ahead at $388^{\prime}$ with no returns. Periodic gel and LCM sweeps.

2300 - Drilling ahead at 412' with no returns. Drilling has been very rough from 388' to $412^{\prime}$; estimate 3000 bbl fluid loss in this interval. Will circulate and work pipe. 


\section{$8 / 14$}

0200 - Cement plug (210 $\mathrm{ft}^{3}$ - \#12) in place at 407'. When drill pipe was pulled out of the hole, fluid level was at $230^{\prime}$, implying that the plug had all escaped into the formation.

0500 - Another cement plug (100 $\mathrm{ft}^{3}$ - \#13). Woc until 1200 .

1200 - Tag cement at 357', very soft; pumped 500 bbl fluid with no returns.

1400 - Pumped cement plug - \#14 - (300 ft at 345').

2100 - Tagged cement at 311'; fluid level at $180^{\prime} ;$ filled hole.

\section{$8 / 15$}

0030 - Fluid level still at $180^{\prime}$ (cement seems to be holding). Begin drilling out cement at $311^{\prime}$.

0400 - Directional survey at $379^{\prime}$; inclination was $3 / 4^{\circ}$.

0600 - Drilling ahead at $412^{\prime}$.

0700 - Lost circulation at $425^{\prime}$.

1400 - Drilling ahead at $475^{\prime}$ with no returns. Rough drilling since $425^{\circ}$ and have pumped away about 1550 bbl of fluid. Will set another plug.

1600 - Cement plug - \#15 - in place. $319 \mathrm{ft}^{3}$ at $438^{\prime}$.

1730 - Tag cement at 426', fluid level at 311'. Pump 300 bbl fluid and get no fill.

2000 - Pump another $310 \mathrm{ft}^{3}$ plug - \#16 - at 421'.

$2400-$ WOC

$8 / 16$

0400 - Tag cement at $409^{\prime} ;$ fluid level at $274^{\prime}$

0600 - Drilled to $430^{\prime}$, so that we can set another plug (\#17) in a cleaned out hole.

1100 - Cement - \#17 - in place (319 $\mathrm{ft}^{3}$ at $\left.425^{\prime}\right)$. Will woc until 1430 .

1430 - Tagged cement at $419^{\prime}$, it was still not cured. will woc three more hours.

1730 - Tried to run temperature tool to find out if low temperature is hindering the cement cure, but the tool would not go through the 
tool joints of the drill pipe. Abandoned that idea and began drilling out cement.

2300 - Drilled out cement to $475^{\prime}$ and did a directional survey at $446^{\prime}$; inclination was $11 / 2^{\circ}$.

\section{$8 / 17$}

0500 - Drilled new formation to 518', drilling extremely hard; did directional survey at $472^{\prime}$, inclination was $31 / 4^{\circ}$.

0630 - Circulated the hole and did another directional survey at 503 '. Survey required 3 tries because of the difficulty of reading the picture. Last result showed an inclination of $3^{\circ}$. Have sent to Bakersfield for an Eastman survey tool to confirm these results (it seems unlikely that a very stiff assembly could have built that much angle in only $30 \mathrm{ft.}$ ) Eastman tools, including a motor for possible directional drilling should arrive about 1100.

1230 - Eastman tools arrive; log complete well, repeating previous surveys. Upper angles are repeatable, but new reading at 472' was 2 $1 / 2^{\circ}$, and $503^{\circ}$ was $31 / 4^{\circ}$. That is, instead of building angle and then dropping back, the deviation was continuously building from approximately $350^{\prime}$. Will plug back to approximately $325^{\prime}$ and directionally drill to straighten out the hole.

1700 - First stage of the cement plug (\#18 - $\left.343 \mathrm{ft}^{3}\right)$ ready to go in the hole.

$2400-$ wOC

\section{$8 / 18$}

0400 - Tagged top of first cement plug at 398', polished top of plug to 412', circulated hole clean and laid second stage - \#19 - of plug. Cement for the second plug (500 $\left.\mathrm{ft}^{3}\right)$ is mixed to be harder than the first stage.

1400 - Tagged top of second plug at $315^{\prime} ;$ circulated/drilled about $5^{\prime}$ off top of plug.

1630 - Cement appears hard enough to drill. Began pulling drill pipe out of hole and picking up motor for directional run.

2130 - Started in hole with Dyna-Drill motor; drilling at about 325' with $1.5^{\circ}$ bent sub. Drill string is rotating at about $25 \mathrm{rpm}$; this is an attempt to keep a straight hole without kicking off in a specific direction. BHA is bit/roller reamer/12" Dyna Drill/bent sub/monel DC/stabilizer/shock sub/3-11" DC/2-10" DC.

2400 - Drilling at $355^{\prime}$. 


\section{$8 / 19$}

0530 - Have drilled to 417'; survey at 357' (because of the motor, reamer, and subs the survey tool can only get to within $60^{\prime}$ of the bit) shows no deviation.

0800 - Drilled to $448^{\prime}$, survey at $388^{\prime}$ shows $3 / 4^{\circ}$ deviation. pulling drilling assembly out of the hole to make a survey nearer bottom.

1130 - Very slow coming out of the hole from $448^{\prime} ;$ basic problem is wrong inserts for the slips, making it difficult to handle the large diameter tools (e.g., the 11" drill collars).

1430 - Finally get a survey at $426^{\prime}$, deviation is $3 / 4^{\circ}$. will lay down the Dyna-Drill and bent sub, and pick up a Christensen Navidrill motor and straight hole drilling assembly. Christensen motor turns at slower speed than the DD (less than $200 \mathrm{rpm}$, as opposed to $300 \mathrm{rpm})$, which is desirable.

1500 - Work on rotary inserts, change BHA, and run in hole.

2030 - Ream from $342^{\prime}$ to $448^{\prime}$.

2400 - Drilling at $464^{\prime}$.

$8 / 20$

0730 - Drilling new formation at $518^{\prime} ;$ lost 450 bbl at $510^{\prime}$, no returns. Cuttings from this interval (before returns lost) contain quartz crystals that only form in open caverns, confirming the presence of large voids. Pull motor out of hole to get survey at $489^{\prime}$; inclination is $12^{\circ}$. Rig for cementing. Sandia logging truck arrives on site.

1030 - Cement plug (\#20-200 $\left.\mathrm{ft}^{3}\right)$ in place at 504'. Will try to fill with fluid at 1300 , tag at 1630 .

1300 - Hole will not hold fluid. Use logging truck to find top of cement at $465^{\prime}$ and attempt to find a loss zone by looking at temperature changes. Get several temperature discontinuities, but they appear to be caused by the separated flow of the cold water going into the hole and the warmer mud already there. Fluid level is at 275' and dropping when logging tool comes out of the hole. Conclude that fluid loss is at the bottom.

1700 - Rig for cementing. Second plug (\#21 - $111 \mathrm{ft}^{3}$ at $\left.460^{\prime}\right)$ in place at 1720 . Echometer shows fluid level holding steady at $279^{\prime}$.

2000 - Tag top of second plug at 449', but hole still will not hold fluid. Temperature survey shows loss zone at about $365^{\prime}$. Will spot cement at about $355^{\prime}$ and try to flow it into the loss zone. 


\section{$8 / 21$}

0830 - Spotted $160 \mathrm{ft}^{3}$ cement - \#22 - (class G, $40 \%$ silica flour) at 347'. This will be a slow cure, will do a temperature log at 1400.

1400 - Temperature $\log$ showed water level at $140^{\prime}$ and cement bridge at $337^{\prime}$. Ran in with drill pipe, and apparently knocked loose whatever was at $337^{\prime}$ (it was fragile, weight indicator showed no force). Fluid level sank, and hole would not fill up.

1800 - Ran in hole with drilling assembly to polish the top of the plug at 448'. Found all the uncured cement from this morning's plug at the bottom of the hole. Circulating with little loss of fluid, have circulated out the last plug. Will run in with the motor after polishing the plug (this plug, which should be well-cured, is not very hard).

2400 - Have reamed out cement to 514' and drilled new hole to 528'.

$8 / 22$

0900 - Have drilled from 528' to 638' since midnight. Have lost and regained circulation twice (total loss about $800 \mathrm{bbl}$ ). Lost circulation again at 638'. Spotted LCM pill. Dry drilled to 646' and spotted LCM.

1200 - Drilled with no returns to $674^{\prime}$, built mud volume, and spotted a lost circulation pill. stili not holding fluid.

1330 - Pulling out of hole with mud motor; will run back in with a monel DC and bit with nozzles pulled. When bit is on bottom, will take a directional survey and then pump a pill of the rubber LCM.

1530 - Pumped a $60 \mathrm{bbl}$ LCM pill with $20 \# / \mathrm{bbl}$ of type 095 rubber; hole still will not hold fluid, but keep pumping into it as we run open-end pipe in for cementing. Will do Echometer to check fluid level $\left(170^{\prime}\right)$.

1630 - Cement plug (\#23 - $\left.319 \mathrm{ft}^{3}\right)$ spotted at $629 \mathrm{ft}$.

1900 - Tagged top of cement at $665^{\prime}$ (9' fill); i.e., most of the cement went into the formation.

2200 - Tried to run temperature log, looking for other loss zones, but cable failure in the logging truck prevented this.

$8 / 23$

0200 - Spotted another cement plug (\$24 - $\left.319 \mathrm{ft}^{3}\right)$ at $617^{\prime} ;$ came back and tagged top of cement at $549^{\prime}$. 
0500 - Spotted an LCM pill of $20 \# / \mathrm{bbl} 095$ rubber, $8 \# / \mathrm{bbl}$ nut plug at 545'. Hole will not hold fluid.

0800 - Pumped another LCM pill of (8\# stringy rubber + 8\# chunky rubber + 6\# Kwick-seal medium)/bbl, chased with $60 \mathrm{bbl}$ water. This appeared to hold water for quite a while (30 min) but then fluid level began dropping.

1100 - Closed diverter and began squeezing LCM. After pumping for approximately 1.5 hours, the hole was holding pressure. stopped pumping and opened diverter; fluid level began dropping.

1300 - Pumped another cement plug - \#25-(319 $\mathrm{ft}^{3}$ at 534'); after pumping that plug, fluid dropped drastically, to about $274^{\prime}$.

1800 - Tagged cement at 474'; pumped another LCM plug of 11\#/bbl 020 rubber, $9 \# / \mathrm{bbl}$ sawdust, and $9 \# / \mathrm{bbl} \mathrm{Kwick}-\mathrm{seal}$ coarse. Pumped mud after this pill, and hole held fluid.

2400 - Reaming cement with mud motor (table locked) at 514'.

$8 / 24$

0200 - cleaning out cement at 540', lost circulation. Continued cleaning to $570^{\prime}$ ' with no returns. Pumped $60 \mathrm{bbl}$ LCM pill (5\#/bbl stringy rubber, $10 \# / \mathrm{bbl}$ coarse rubber, $10 \# / \mathrm{bbl} \mathrm{Kwick-Seal} \mathrm{coarse)} \mathrm{at}$ $558^{\prime}$.

0500 - Rig to cement - \#26 - at 560'; $319 \mathrm{ft}^{3}-2: 1$ perlite, $12 \%$ Cal-seal, 3\% CaCl.

1200 - Tagged top of cement at 511..

1500 - Cleaned out cement from 511' to 580'; lost circulation at $580^{\prime}$.

1800 - Continued cleaning out cement to $658^{\circ}$ (no returns). Stopped to rig for cementing.

2100 - Cement - \#27 - with diverter tool at 655' while working dtool from $628^{\prime}$ to $658^{\prime}$; run $60 \mathrm{bbl}$ water, $60 \mathrm{bbl}$ sepiolite, $421 \mathrm{ft}^{3}$ cement (1:1 perlite, $40 \%$ silica flour, 6\% Cal-seal, 3\% CaCl). pulled up out of cement $110^{\prime}$ and laid on $30 \mathrm{bbl}$ mud.

2310 - Cement in place.

$8 / 25$

0400 - Tagged top of cement plug at 590', but hole would not hold fluid (fluid level at $390^{\prime}$ ).

0730 - Set $434 \mathrm{ft}^{3}$ cement plug - \#28 - (2:1 perlite, 12\% Cal-seal) at 590'. Hole is holding fluid. 
1200 - Tagged top of plug at 500', indicating that some of the cement went into the formation. RIH with mud motor.

1600 - Drilled about $7^{\prime}$ of cement and it was still soft; will wait for 2 hours.

1800 - Drilled from $507^{\prime}$ to $563^{\prime} ;$ complete loss of returns. continued drilling to $582^{\prime}$.

1930 - Pumped $60 \mathrm{bbl}$ LCM plug, loaded $60 \mathrm{bbl}$ water on top, waited 20 minutes and resumed circulation with no returns.

2300 - Pulled out of hole, ran in with open-ended drill pipe, and spotted $20 \mathrm{bbl} \mathrm{CaCl}$ water and $20 \mathrm{bbl}$ "Super Plug" in a 10C-10sP-10ClOSP sequence with water spacers between each pill. At the same time, pumped $5 \mathrm{bbl} / \mathrm{min}$ water in the annulus to try to drive the LCM into the formation. Wait 30 minutes and try to fill hole, with no success.

\section{$8 / 26$}

0030 - Rig for cementing; led cement - \#29 - with 2000 gal Flo-chek, then $256 \mathrm{ft}^{3}$ 1:1 perlite, $12 \% \mathrm{Cal}$-seal, $3 \% \mathrm{CaCl}$ at 569'. Pumping 5 $\mathrm{bbl} / \mathrm{min}$ water into annulus at the same time.

0700 - Tagged top of cement at 515', pumped $400 \mathrm{bbl}$ water, and hole would not hold fluid.

0800 - Rigged logging truck to do temperature survey, but hole was full. Rig crew had continued pumping through the fill line to make room in the mud tanks for an LCM mix; this additional fluid had been retained for some unknown reason. (The additional pumping, which we had not known about, would have made the temp log meaningless anyway.)

0900 - Mixing approximately 1000 bbl mud with $35 \%$ medium Kwick-Seal. will lay the motor down, and try to drill ahead with the drilling assembly and circulate the hole clean of the cement and other noncutting materials.

1400 - Have not tried to drill yet, but are doing another cement job - \#30 - just above the previous plug (356 $\mathrm{ft}^{3}$ of class G, 3\% CaCl).

1700 - Run in hole with drilling assembly, tag top of cement at $431^{\prime}$. WOC

2300 - Begin drilling out cement at 431'.

2400 - Drilling cement at $440^{\prime}$. 
0800 - Have drilled out cement from 440' to 497'. Two directional surveys; $411^{\prime}=11^{\circ}, 442^{\prime}=3 / 4^{\circ}$. Circulating mud over shakers to clean up mud system.

1100 - Drill to $534^{\prime}$, directional survey at $506^{\prime}=1 / 2^{\circ}$.

1400 - Drilling cement at $65^{\prime}$ (no cement or fill in the hole from $572^{\prime}$ to $\left.600^{\prime}\right)$; directional survey at $600=1 / 2^{\circ}$

1730 - Drilling new formation at 675'

2100 - Drilling at $698^{\prime} ;$ directional survey at $663^{\prime}=1^{\circ}$. Pulling out of hole to lay down drilling assembly and pick up Eastman motor because of low ROP $=8 \mathrm{ft} / \mathrm{hr}$.

\section{$8 / 28$}

0400 - Drilling at $765^{\prime} ;$ directional survey at $695^{\prime}=3 / 4^{\circ}$.

0800 - Drilling at $828^{\prime}$; directional survey at $758^{\prime}=1 / 2^{\circ}$.

1000 - Drilling at $891^{\prime} ;$ directional survey at $831^{\prime}=1^{\circ}$.

1200 - Drilling at 923'; 235 bbl circulation loss at 929'. Drill ahead, build mud volume, pump LCM pill, regain normal circulation.

1410 - Drilling at $984^{\prime}$; complete loss of returns. Continue drilling and regain returns - steady loss of fluid, because we are constantly building mud volume. Water level is rising in the water well, indicating that the wells are communicating.

1600 - Drilling at $1016^{\prime}$; directional survey at $926^{\prime}=3 / 4^{\circ}$. Still losing fluid at $1048^{\prime}$, will stop drilling and pump cement.

2000 - Spotted cement plug - \#31 - at $1044^{\prime} ;$ led with $20 \mathrm{bbl} \mathrm{CaCl,}$ then 2000 gal Flo-chek, 300 sks Class G, $3 \% \mathrm{CaCl}$, then 135 sks $1: 1$ perlite with $12 \%$ Cal-seal, $3 \% \mathrm{Cacl}$. Total volume $=676 \mathrm{ft}^{3}$.

\section{$8 / 29$}

0030 - Ran temperature log to find top of cement and check for crossflow. Tagged top of cement at $934^{\prime}$, but it appeared to be soft.

0600 - Tagged top of cement at 945' with drill pipe, and it seemed hard.

1300 - Completed drilling cement at 1048'.

1600 - Drilled to $1118^{\prime} ;$ directional survey at $1039^{\prime}=1^{\circ}$. Rig is temporarily down because one of the mud pumps is not working. 
1800 - Mud pump repaired, drilling ahead.

2130 - Drilling at $1207^{\prime}$; directional survey at $1139^{\prime}=3 / 4^{\circ}$. Have lost and regained circulation several times in this interval.

$8 / 30$

0200 - Drilled to $1302^{\prime} ;$ survey at $1233^{\prime}=10$

0600 - Drilled to $1397^{\prime} ;$ survey at $1324^{\prime}=11 / 4^{\circ}$

1200 - Drilled to $1491^{\prime} ;$ survey at $1431^{\prime}=10$

1800 - Drilled to $1573^{\prime} ;$ lost all rig electrical power as both diesel/generator sets failed. Probably caused by a loose wire on one governor, resulting in power surges and overheating. Power surge also apparently killed water well pump.

2000 - Power restored. Drilling assembly was on bottom for at least 20 minutes with no movement or circulation, but came free when power returned. Drill to $1585^{\prime}$, survey at $1525^{\prime}=11 / 8^{\circ}$

2400 - Drilled to 1604 '; rate of penetration is relatively low (around $15 \mathrm{ft} / \mathrm{hr}$ ) because driller cannot put much weight on bit without deviation. With less than 10,000\#, deviation stays under 10 , but weight increases consistently bring deviation increases.

$8 / 31$

0530 - Drill to $1690^{\prime} ;$ survey at $1620^{\prime}=114^{\circ}$

0700 - First load of 20" casing arrives on site, remainder will follow at approximately 1 hour intervals. Eight loads (67 joints total).

1100 - Drill to $1774^{\prime} ;$ survey at $1714^{\prime}=114^{\circ}$

1600 - Drill to 1803'; pull out of hole to run wireline temperature log. Log showed some temperature variation, but the average was around $123^{\circ} \mathrm{F}$. Most of this heat probably came from the mechanical work of the mud pumps and the mud motor. That is confirmed by the fact that the temp probe was cooling as it rested on bottom.

2100 - Drilled to $1835^{\prime}$, lost 400 psi pump pressure and suspected a washout. Pulled drillstring to examine it, found no damage, and located a blockage in the mud pump suction line. Ran back in hole.

$9 / 1$

0530 - Drill to 1840'; circulate thick mud that is building up from the fines of drilling in the ashy formation.

0800 - Drill to $1866^{\prime} ;$ directional survey $=11 / 4^{\circ}$ 
1400 - Drill to 1961'; directional survey $=114^{\circ}$

1600 - Talked with Wayne Jackson (Jackson Equipment Co) and Boyd Green, M-I, about using Jackson's equipment to clean up the solidsladen fluid in \#2 sump. This process stirs up the sludge with a small dredge, chemically flocculates it, centrifuges it to separate the solids, and returns clear water to the sump. Estimated cost to process the waste is $\$ 7 / \mathrm{bbl}$, but there aren't any feasible alternatives.

2400 - Drill to $2044^{\prime}$.

$9 / 2$

0100 - Deviation survey at $1984^{\prime}=114^{\circ}$

0300 - Pressure oscillations and reduced drilling rate indicate that mud motor may be washed out. Lay down Eastman motor and pick up Dyna-Drill. Stage back to bottom, trying to reduce wall cake.

0900 - Back on bottom, but rate of penetration is very low ( 3 $\mathrm{ft} / \mathrm{hr})$. At least two possibilities: we are now in the harder Bishop Tuff, and the bit may be balled from the excessive wall cake. Will try circulating some Nut-plug to clean up the bit.

1200 - Rate of penetration has picked up some, to about $8 \mathrm{ft} / \mathrm{hr}$, and we are definitely in Bishop Tuff at $2060^{\circ}$.

2000 - Drilled to $2125^{\prime} ;$ directional survey $=11 / 2^{\circ}$

2200 - Begin pulling out of hole to lay down mud motor.

\section{$9 / 3$}

0200 - Laid down mud motor and made up new BHA. Will ream back to bottom with "new" bit (previously used, laid down to repair lubricant leak).

0800 - Drilling at $2134^{\prime}$, still at less than $10 \mathrm{ft} / \mathrm{hr}$.

1130 - Drill to $2165^{\prime}$ with $30-35 \mathrm{Klb}$ WOB, survey at $2124^{\prime}=15 / 8^{\circ}$. Appear to be drilling through inhomogeneous formation, drilling rate is fluctuating with constant weight on bit.

1500 - Drilling at $2195^{\prime}$; rate of penetration is down to $5 \mathrm{ft} / \mathrm{hr}$, may be in a glass flow. Survey at $2145^{\prime}=11 / 8^{\circ}$

1700 - Coming out of hole to pick up more drill collars.

1800 - Laid down old three-point reamer (severely worn); picked up new reamer and new shock sub. Picked up 5 ea $10 "$ drill collars.

2400 - Drilled to $2207^{\prime}$. 


\section{$9 / 4$}

0200 - Drill to $2251^{\prime} ;$ survey at $2211^{\prime}=11 / 2^{\circ}$

0600 - Drill to 2314'; survey at $2254^{\prime}=11 / 2^{\circ}$. Rate of penetration has picked up to about $20 \mathrm{ft} / \mathrm{hr}$ with $35-40 \mathrm{KIb}$, rock is showing more quartz crystals (i.e., more like the Bishop Tuff should look).

1000 - Drill to $2377^{\prime} ;$ survey at $233^{\prime}=1^{\circ}$.

1700 - Drill to $2503^{\prime} ;$ survey at $2463^{\prime}=34^{\circ}$.

2100 - Drilling rate is erratic, varying from 6 to $30 \mathrm{ft} / \mathrm{hr}$. Do not want to spend too much time getting deep enough to use all the casing, so will stop at the next even joint.

2345 - TD at 2568'.

\section{$9 / 5$}

0100 - Survey at $2511^{\prime}=3 / 4^{\circ}$.

0300 - Strap out of hole; measurement shows depth indicator 1.5'

off. Run back in hole and drill $2^{\prime}$ to true $2568^{\prime}$.

0600 - Circulate hole and pull out tools. Rig for logging; will do dual induction with gamma, sonic, caliper, and temperature.

1000 - Begin logging runs.

2000 - End logging; sonic logs are doubtful, apparently no returns in some sections of the hole. Could be caused by tool problems, large hole, and/or garbage formation (cement pockets, washouts). Run back in and begin circulating to condition hole.

\section{$9 / 6$}

0500 - Out of hole; begin rigging to run casing.

0800 - Float shoe and float collar screwed and welded in place, two joints apart. Begin running in casing, using a casing stabber yoke to align casing when it is screwed together. Seems to be working well, averaging about 6 minutes per joint.

1430 - Last joint of casing in place. Begin unrigging casing crews and rigging cementing. will run drill pipe into float collar at bottom of casing and begin circulating mud to make sure annulus is clear before cementing.

1815 - Halliburton cementing tree assembled; will stab into float collar and begin circulation. Casing shoe at 2559'. 
2030 - Two hours circulation gives clear returns. Will pull drill pipe, centralize casing, re-run drill pipe with new o-rings on stabin adaptor, and begin pumping cement.

\section{$9 / 7$}

0600 - Cementing complete, $7243 \mathrm{ft}^{3}$ total; lost returns twice, but regained them. Full returns to surface at the end of the job. Will let cement begin curing and wait to see if the top of cement falls back.

1200 - Logged water well; good news is that it shows no change in temperature since baseline log last night (i.e., no cement in water well. Nipple down 30" diverter stack.

1430 - Cement level in annulus has fallen back to approximately 160$180 \mathrm{ft}$. Will do a top job, pumping through fill line. Temperature logging wellbore.

1600 - Top job complete; $277 \mathrm{ft}^{3} 1: 1$ perlite with $40 \%$ silica flour, then pumped $296 \mathrm{ft}^{3}$ class $\mathrm{G}$ with $40 \%$ silica flour. Will woc until 0600 .

\section{$9 / 8$}

0500 - Cut off 20" casing, nipple down diverter and cut off 30" spool and casing.

1000 - Weld on 20" flow nipple and flow line.

1400 - Pick up 17-1/2" bit and run in hole to drill out cement and float collar in preparation for wireline coring.

1800 - Drill cement from $2474^{\prime}$ to $2522^{\prime}$.

2300 - Pull out of hole and lay down drill pipe and collars.

\section{$9 / 9$}

1000 - Pick up 5-7/16" sHR core bit, 17-1/2" nonrotating stabilizer and special ocean Drilling Program 5" drill pipe.

1600 - circulate mud out of hole with water.

1800 - Drill cement with core bit to anchor drill pipe from $2527^{\prime}$ to $2534^{\prime}$.

2200 - Circulate to clean hole.

\section{$9 / 10$}

0000 - Pull drill pipe from hole to remove stabilizer and nipple down flow line. 
0300 - Install and weld 20" wellhead using "Hot-Head" pre-heat and post-heat tools. Test welds with gas to $200 \mathrm{psi}$ at $750^{\circ} \mathrm{F}$ after

post-heat.

1400 - Install 20"x10" companion flange and 10" tubing hanger spool.

1600 - Ran in hole with SHR core bit on 5" ODP drill pipe. Landed drill pipe in the tubing spool with a double box hanger. This leaves the core bit at 2532', 5' into and $2^{\prime}$ off bottom of anchor hole.

1800 - Nipple up 10" 3000 psi master valve.

1900 - Clean mud tanks.

\section{$9 / 11$}

1000 - Pressure test casing and wellhead to 800 psi for 15 minutes, no leaks. Continue cleaning mud tanks and location.

\section{$9 / 12$}

1600 - Finished cleaning tanks and location. Released rig and crew, Jackson equipment still cleaning waste sumps. Will start rigging up for coring on $9 / 25$. Location shut down except for Jackson's sump cleaning.

\section{$9 / 25$}

0800 - Tonto Drilling CP-50 electric/hydraulic core rig on site. This rig will be powered by a $200 \mathrm{hp}$ electric power pack powered by the Loffland rig. It will be mounted on the Loffland rig floor and will use a sheave wheel suspended from the traveling block to allow rod handling with the CR-50 drill hoist. See Figure 2 for a schematic of core rig operation.

0900 - Crane on site to set power pack, core rig, core rods and tools on rig floor. Welder on site to tie core rig down to rotary table and floor. Crane also setting core rig mud pumps and tanks on drill pad below rig floor.

1400 - Check out CP drill and mud system, all check OK. Rig up complete. This went very well.

1600 - Nipple up 4" Hydril, choke and kill lines.

1800 - Run core barrel in hole and test BOP equipment. Will shut down until tomorrow daylight tour. Then will start 24 hour operation. Each tour will consist of a two man crew; one Tonto core driller, with a Loffland driller to operate Loffland equipment and help the core driller. A Loffland rig superintendent will also be on duty. 
0800 - start tripping in coring rods in $10^{\prime}$ lengths.

1000 - Test BOP and rig equipment. Test witnessed and approved by BIM.

2000 - Finish tripping in and start coring and washing cement at $2522^{\prime}$.

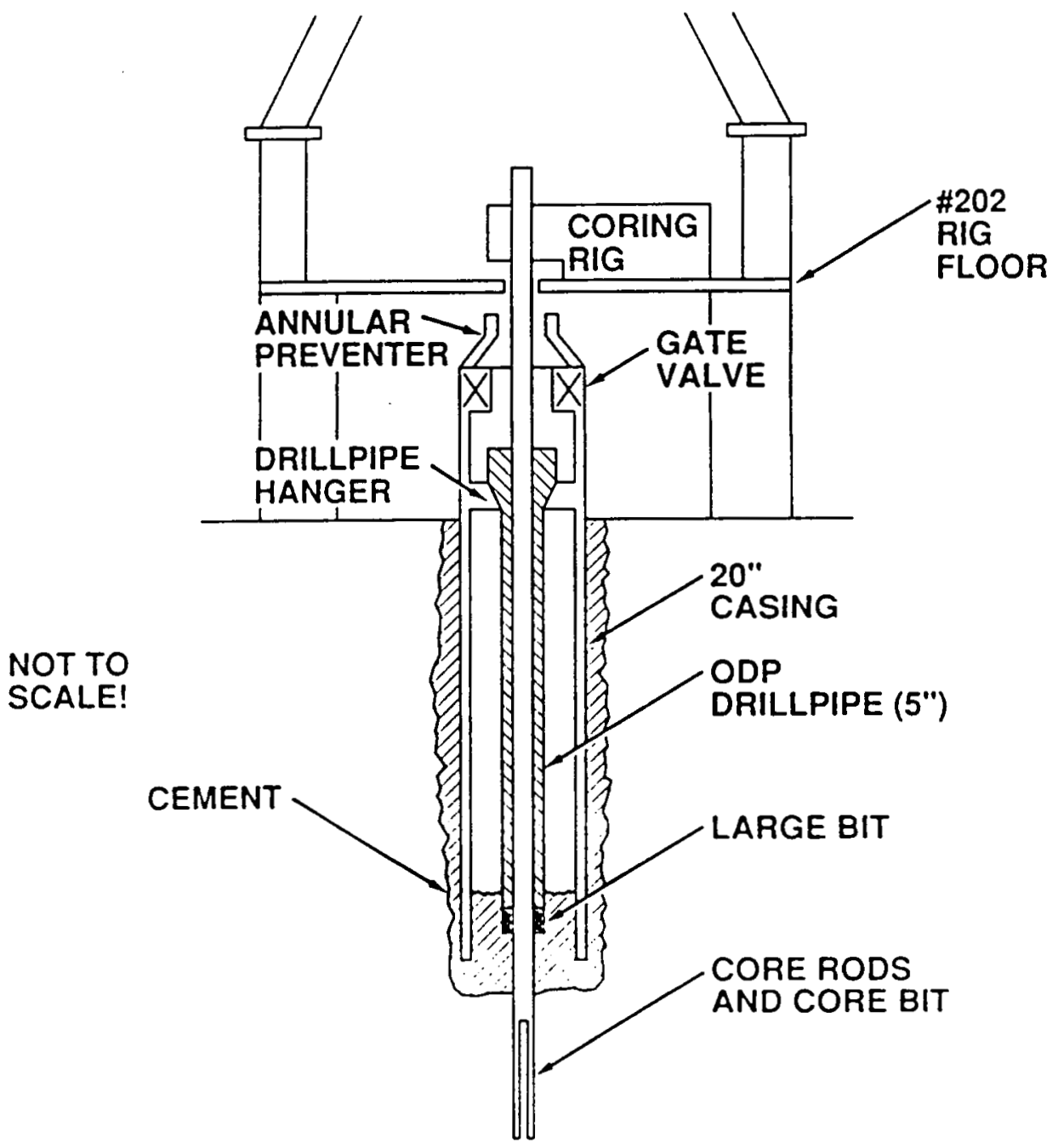

Figure 2 -- Schematic of core rig operation. Core rig is placed on large rig's floor; core rods travel inside ODP drill pipe, which acts as an "artificial wellbore". 
0700 - Cored to 2572', retrieved float shoe valve at $2556^{\prime}$ and new formation at $2568^{\prime}$.

0800 - Bit plugged, cannot break circulation, may have dropped some core. Will pull drill rods to unplug bit.

1430 - Out of hole, bit plugged with heavy coarse sandy material and LCM.

1500 - start tripping in hole.

2100 - at $2550^{\prime}$ start washing hole with fresh mud and wash to $2572^{\prime}$.

2200 - Start coring new formation, core from 2572' to 2576'. Cored $4 "$ and recovered all of it.

$9 / 28$

0800 - Going good, cored to $2624^{\prime}$ with complete recovery. 2000 - Core driller shift change, cored $60^{\prime}$ to $2684^{\prime}$; got it all.

$9 / 29$

0130 - still coring, at $2724^{\prime}$ but losing some returns.

0800 - Now getting full returns. Cored to $2744^{\prime}$ ' with better than $99 \%$ recovery. Change out mud to remove solids that are causing mud rings in pipe.

1100 - After coring to $2754^{\prime}$ and pulling inner barrel the core pipe became stuck. Cannot circulate, rotate, pull up or push down. Core bit is $2^{\prime}$ off bottom.

1800 - Working stuck pipe, will take some fluid at high pressure but no returns. Have tried pulling, pushing, surging and rotating, but cannot move. Will try just leaving them until $2400 \mathrm{hrs}$ and then try again.

\section{$9 / 30$}

0300 - Still working stuck pipe, broke circulation with high pressure and are getting a very small return. Pipe moved down 1/2' but still stuck. Pumping down soap to help free pipe.

0900 - Applied reverse torque to core rod and unscrewed it about 400 'down. Blew air down core rods to unload fluid above break. Made up core rod joint but still no luck. Continued working pipe and called out loggers to run free point log and cut pipe.

2130 - Loggers arrive. Rig for free point log. 
2230 - Log indicates rods stuck at $2644^{\prime}$ and free at $2634^{\prime}$. Will jet cut rods at $2520^{\prime}$. This will leave the top of the core rods in the ODP pipe above the cement level in the 20 " casing.

$10 / 1$

0100 - Rig down loggers. Start tripping out and laying down core rods in $10^{\prime}$ lengths.

1000 - Finished tripping out with core rods. Rod tally indicates top of jet cut at 2511.2' and cut was clean. The hole TD is 2754'.

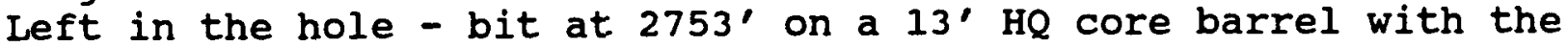
inner tube removed, 22 whole $10^{\prime}$ HMQ core rods and one $8^{\prime} 5^{\prime}$ cut HMQ rod. Cut is at $2511.2^{\prime} \mathrm{KB}$.

1100 - start displacing drilling fluid in hole with water and corrosion inhibitor. Start removing coring equipment from rig floor.

1400 - Corrosion inhibitor circulated through ODP pipe and casing. Air lifted fluid to leave fluid level at $234 \mathrm{ft}$. Shut in well and secure.

1800 - Finished highlining core rig, power pack, rods and tools from rig floor. Shut down operation until daylight shift tomorrow.

\section{$10 / 2$}

1000 - Finished packing coring equipment, cleaning core mud system, nippling down BOP, and securing equipment for the winter.

1100 - Released Loffland and Tonto rigs. Jackson equipment still cleaning sumps but we are not generating any more drilling fluids.

\section{PHASE I COMPLETE}

[See appendices for detailed listing of LCM, drilling fluid additives, rig time breakdown, bit records, and daily reports] 
bbl - barrel; standard unit of fluid measure in drilling practice, equals 42 standard gallons.

BHA - bottom hole assembly; the combination of bit, stabilizers, drill collars, and other tools at the bottom of the drill string, i.e., everything below the drill pipe.

DC - drill collars; the heavy-walled tubular sections of the drill string used just above the bit to apply weight.

h.o. - hole opener; a cutter used above a bit to enlarge the hole diameter.

$\mathrm{KB}$ - kelly bushing; the reference level at the rig floor from which all depths are measured.

LCM - lost circulation material; particulate matter mixed with the drilling fluid to plug loss zones in the formation.

RIH - run in hole; tripping the drill string into the hole.

ROP - rate of penetration; the rate at which the bit is advancing.

wOB - weight on bit; the force applied to the bit by the weight of the drill string components.

WOC - wait on cement; time spent waiting for cement to cure. 
APPENDIX A

DETAILS OF TIME FOR VARIOUS RIG ACTIVITIEs (Coring not included)

ACTIVITY HOURS PERCENT

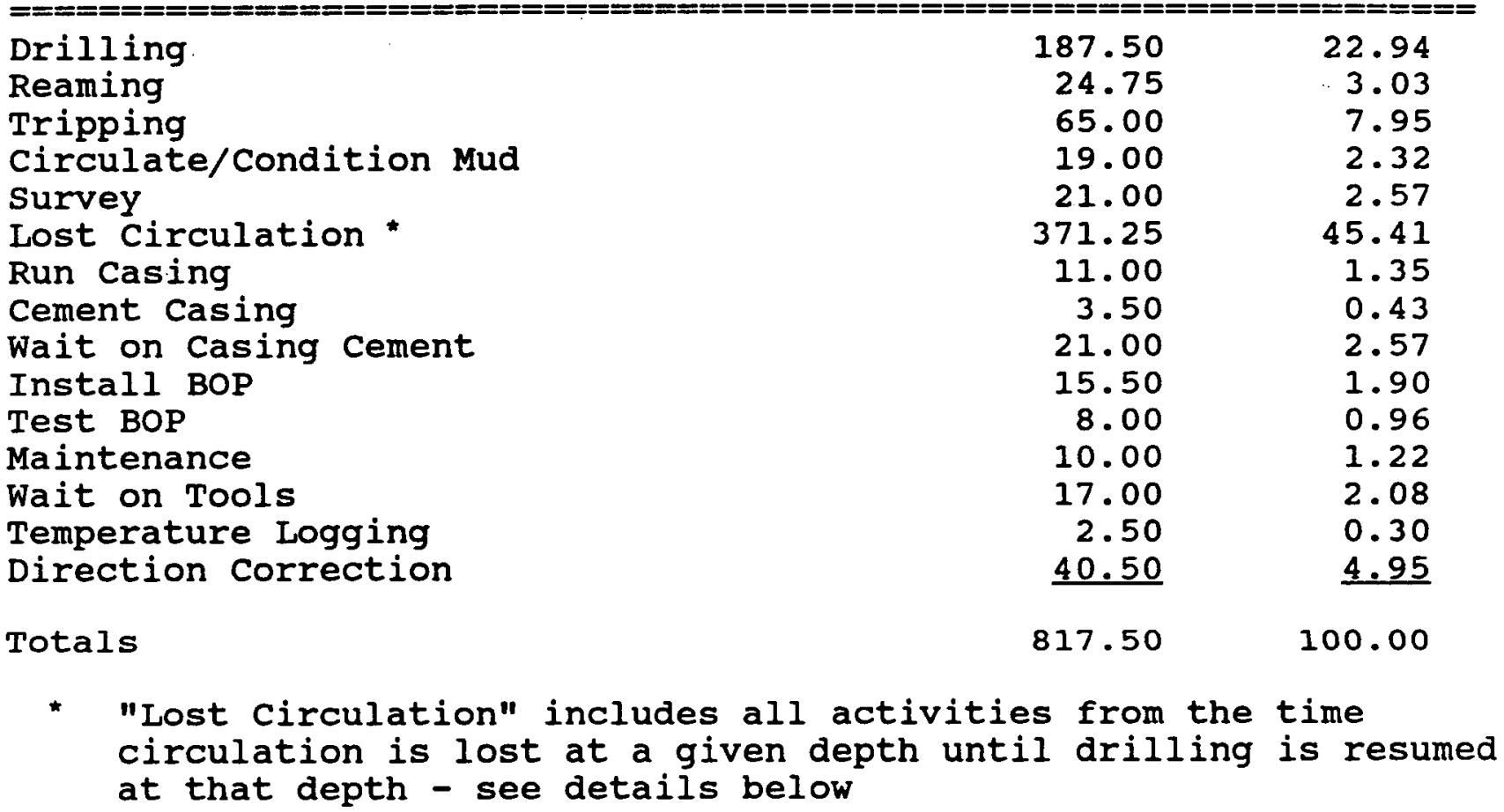

DETAILS OF TIME FOR SPECIFIC LOST CIRCOLATION ACTIVITIES

ACTIVITY

$============$
Build Mud Volume

Circulate/Pump LCM

Trip

Rig Up/Mix Cement/Cement

Wait on Cement

Drill/Ream Cement

Log/Survey

other

Totals
HOURS

6.75

37.00

68.50

28.25

154.25

55.00

12.00

9.50

371.25
100.00

PERCENT

1.82

9.97

18.45

7.61

41.55

14.81

3.23

2.56 


\section{APPENDIX B}

\section{BIT RECORD}

Bit footages are divided into four categories:

$$
\begin{aligned}
& \mathrm{N}=\text { normal rotary, drilling rock } \\
& \mathrm{C}=\text { normal rotary, drilling cement } \\
& \mathrm{M}=\text { mud motor, drilling rock } \\
& \mathrm{CM}=\text { mud motor, drilling cement }
\end{aligned}
$$

\section{BIT TYPE}

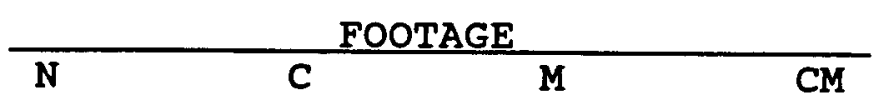

Smith DSJ

Smith 2JS

security s3sJ

Security 584

$\begin{array}{rr}393 & 329 \\ 508 & 243 \\ 44 & 296\end{array}$

507

649

1039

102

Note: In a hole with $T D=2568^{\prime}$, total cement drilled $=1619^{\prime}$. 


\section{APPENDIX C}

\section{BRIEF BPECIFICATIONS FOR LOFFIAND RIG \$202}

The Loffland Brothers rig used for this drilling has drilled the two deepest holes (both below 30,000 feet, both in Oklahoma) in the United states. Some of the rig capacities are listed below.

* Mast capacity: 1000 tons

* Height to crown: 177 feet

* Maximum hook load: 750 tons as rigged $(30,000 \mathrm{ft}$ of 5 " drillpipe): can be rigged for 1000 tons

* Pump capacity: 2 pumps; 736 gpm each at 3260 psi

* Drill pipe: 5-1/2"; 5-1/2" full hole tool joints

* Prime movers: 3 ea Catepillar D-399 $1100 \mathrm{hp}$ diesels

* Mud system: 1400 barrel capacity; shakers, desanders, desilters

\section{BRIEF SPECIFICATIONS FOR THE TONTO CP-50 CORING RIG}

The Tonto $\mathrm{CP}-50$ is built as an underground diamond coring rig. Although it is often truck-mounted and used with a mast for surface drilling, it was mounted for this application on the Loffland rig floor and powered by the Loffland rig generators.

* Manufaturer: Chicago Pneumatic

* Powerpack: $200 \mathrm{hp}, 460$ volt, 3 phase electric motor

* Drive: Hydrostatic

* Maximum single line pull: 30,000 1b

* Depth capacity: $11,000 \mathrm{ft}$

* Drill pipe: HMQ wireline drill rods, 3-1/2" OD x 3-1/16" ID

* Pump capacity: 2 pumps; $35 \mathrm{gpm}$ each at 1000 psi

* Mud system: 900 gallon mud tank, with 300 gallon mixing tank 
APPENDIX D

DRILLING FLUID ADDITIVES AND LOST CIRCULATION MATERIALS

ADDITIVES :

QUANTITIES

VISCOSIFIERS-

M-I GEL

POLY PLUS

$451,5001 \mathrm{~b}$

$75 \mathrm{gal}$

FLUID LOSS ADDITIVE-POLYPAC

$10001 \mathrm{~b}$

THINNER-TANNATHIN

$14,0001 \mathrm{~b}$

SURFACTANTS-

DMS

LUBE-106

SURFAK M

$480 \mathrm{gal}$

$130 \mathrm{gal}$

55 gal

DEFOAMERS-

ALUMINUM STEARATE

DEFOAM-L

DEFOAM-X

$2251 b$

$140 \mathrm{gal}$

25 gal

OTHER COMMERCIAL CHEMICALS-

CAUSTIC SODA

IMCO SAPP

LIME

M-I BAR

SODIUM BICARB

$$
\begin{array}{rr}
2450 & 1 b \\
2600 & 1 b \\
3150 & 1 b \\
800 & 1 b \\
39,200 & 1 b
\end{array}
$$

LOST CIRCULATION MATERIALS:

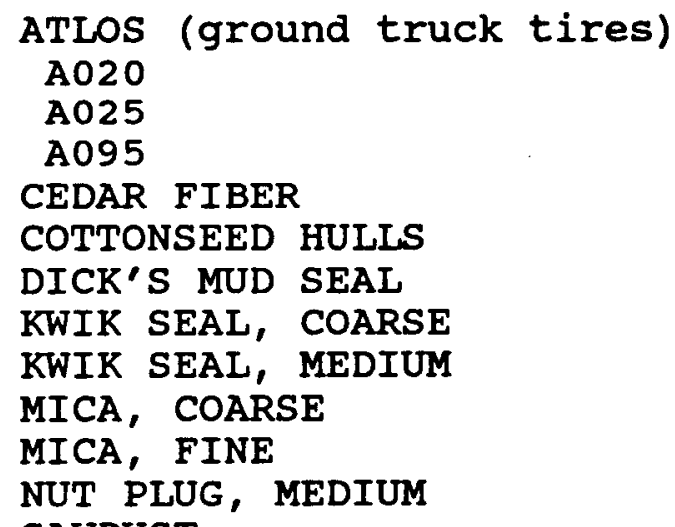

SAWDUST

TOTAL LCM 


\section{APPENDIX E}

TEMPERATURE LOGB

The following temperature logs were made after completion of the coring operation. That is, the temperature sensor was lowered inside the drill pipe and core rods that are still in the hole. 
TEMPEAATUAE IN DEG. C.
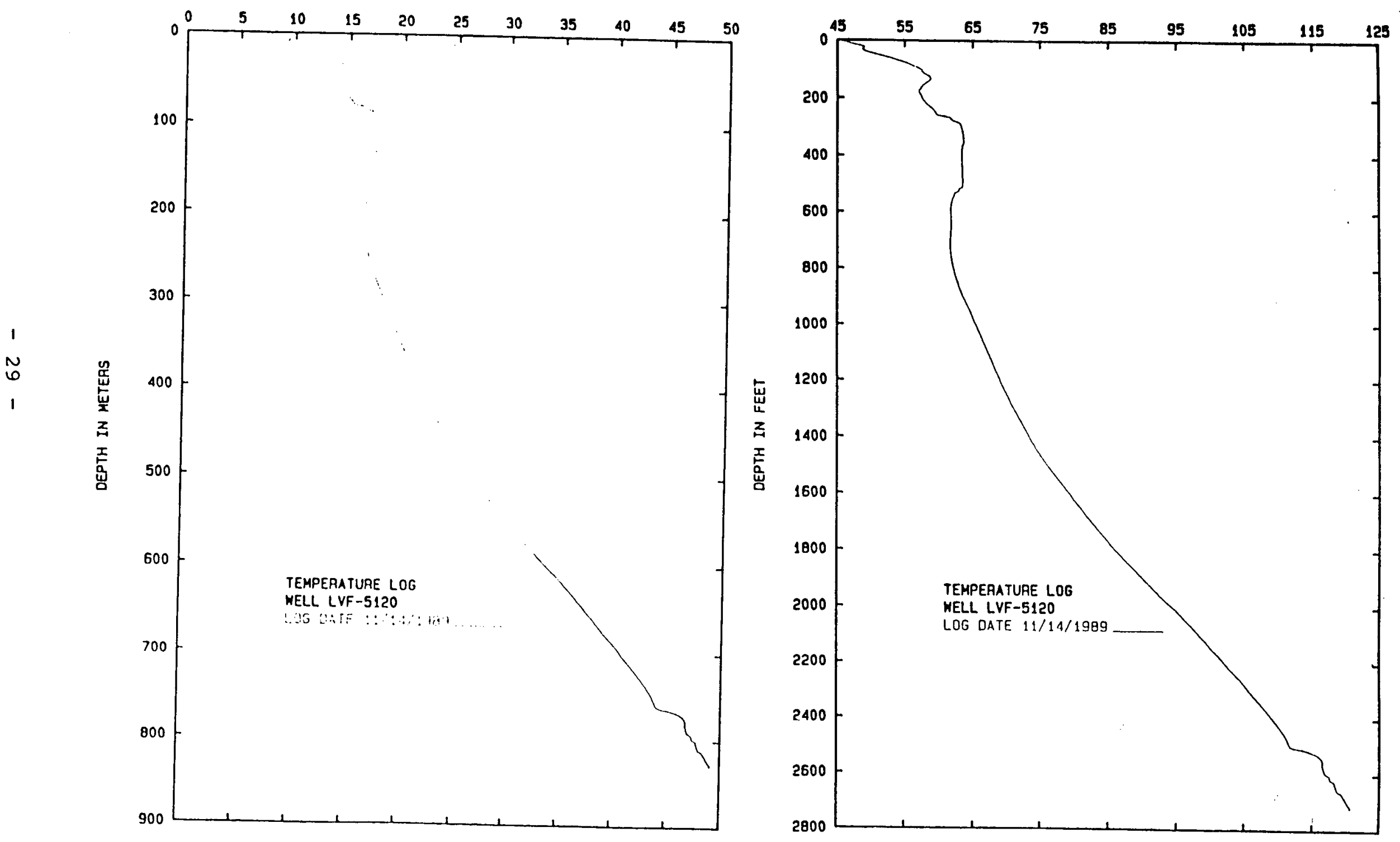


\section{APPENDIX F}

\section{DIRECTIONAL LOGS}

The following directional survey was done after the completion of coring. Survey tool travel was through the drill pipe and core rods. 


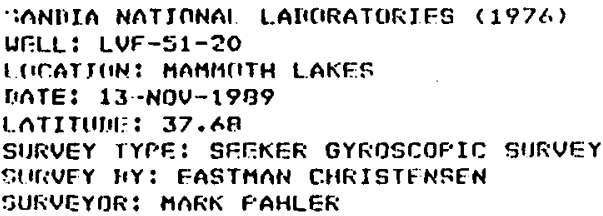

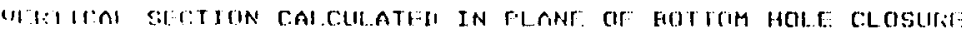

RECDFU DF SUFUEY

FALITUS GI CURUATURE METHOH

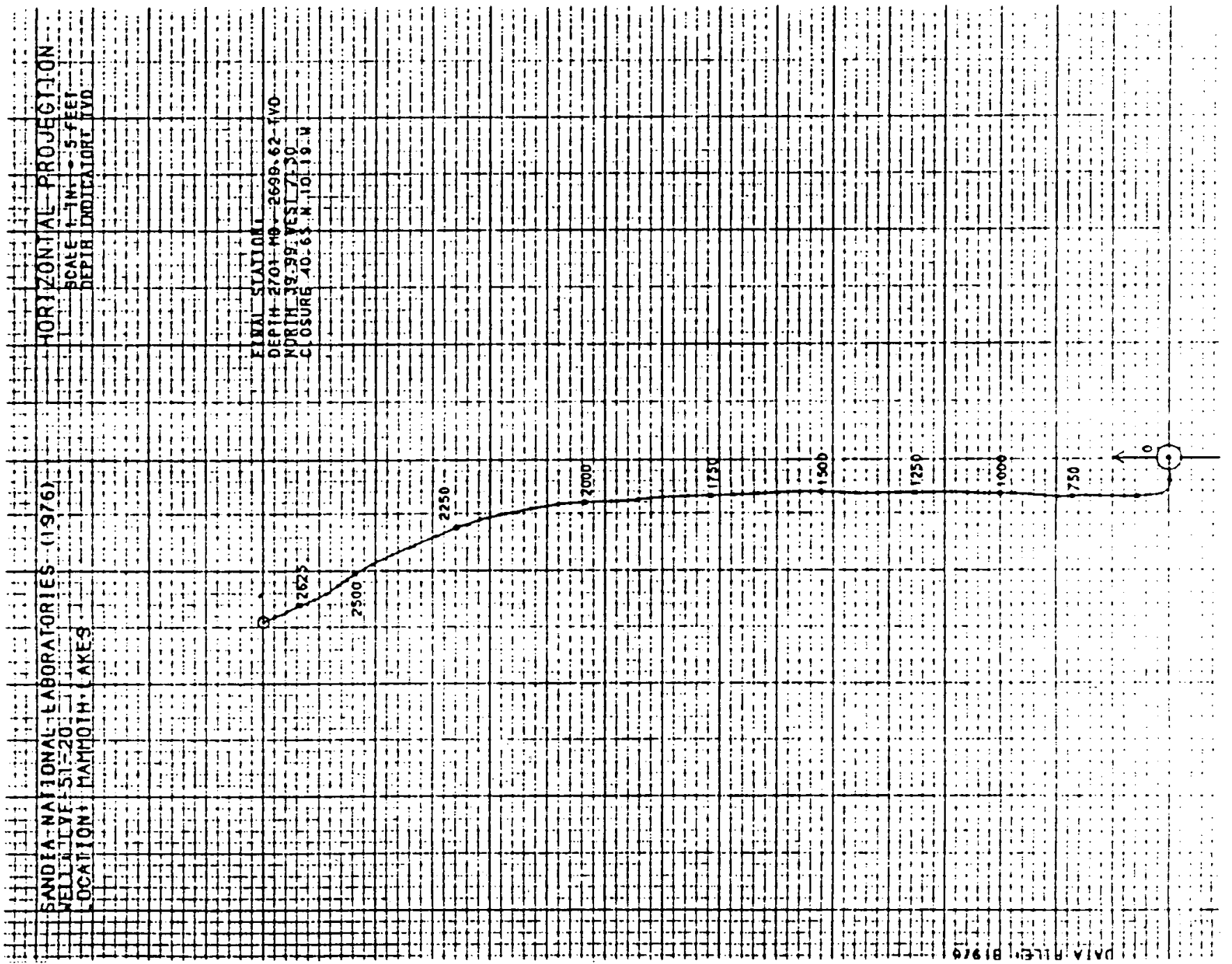




\begin{tabular}{|c|c|c|c|c|c|c|c|c|c|}
\hline$\therefore$ & & & & & & & & TRUE: & \\
\hline MEASUREU & tin & IFT & & DRI & ET & & COURSE & VERT ICAL & VERT I CAL \\
\hline "uF:Tt" & AN & GLE & & KEEC & TTIC & & LENGTH & IEPTH & SECTION \\
\hline FFET & , II & M & & $\mathbf{n}$ & M & & & FEET & FEET \\
\hline o. & 0 & 0 & & 0 & 0 & & 0. & 0.00 & 0.00 \\
\hline 50 & 0 & 13 & $\mathbf{s}$ & 76 & 51 & $\omega$ & 5o. & 50.00 & -0.00 \\
\hline 100. & 0 & 13 & $N$ & 76 & 11 & $W$ & 50. & 100.00 & 0.03 \\
\hline 150. & 0 & 20 & 5 & 78 & 47 & $W$ & $\begin{array}{l}50 . \\
50\end{array}$ & $\begin{array}{l}150.00 \\
200.00\end{array}$ & 0.08 \\
\hline 200. & 0 & 20 & $\mathbf{S}$ & BA & & W & & & 0.09 \\
\hline 250. & 0 & 18 & $N$ & 80 & 58 & $\omega$ & 50. & 250.00 & 0.14 \\
\hline 2000 . & 0 & 15 & $N$ & e7 & 12 & W & $\mathrm{so}$ & 300.00 & 0.21 \\
\hline 350. & 0 & 16 & N & 60 & 24 & $\mathbf{W}$ & 50 & 350.00 & 0.32 \\
\hline 100. & 0 & 31 & $N$ & 3 & 29 & $W$ & 50 & 400.00 & $0.4:$ \\
\hline $4 \div 0$. & 0 & 38 & $N$ & 9 & 18 & $w$ & 50 & 449.99 & 1.12 \\
\hline 500. & 0 & 40 & $N$ & 0 & 2 & $E$ & 50. & 499.99 & 1.68 \\
\hline 550. & 0 & 3.5 & N & 0 & 1 & $\mathbf{W}$ & so. & 549.99 & 2.22 \\
\hline 1.000 & 0 & 10 & $N$ & 3 & 5 & E- & 50. & 595.90 & 2.76 \\
\hline 650. & 0 & 41 & $N$ & 0 & 40 & E & so. & 647.98 & 3.34 \\
\hline 7010. & 0 & 40 & $N$ & 0 & 24 & $E$ & 50. & $0.9 \% .98$ & 3.92 \\
\hline 750. & 0 & $1: 3$ & H & 5 & 57 & W & 50. & 749.37 & 4.52 \\
\hline$:: 10$. & 0 & 40 & $N$ & 1 & 25 & $E$ & 50. & 799.97 & 5.11 \\
\hline B5\%. & 0 & 16 & N & 8 & 14 & $E$ & so. & 849.97 & 5.72 \\
\hline 900. & 0 & 47 & $\mathbf{N}$ & 2 & 29 & $E$ & 50. & 899.96 & 6.37 \\
\hline 950. & 0 & 14 & $H$ & 3 & 47 & $E$ & so. & 919.96 & 7.02 \\
\hline son, & 0 & 18 & $N$ & 2 & 24 & $w$ & 50 & 999.95 & 7.67 \\
\hline 1050 & 0 & : & $\mathbf{N}$ & 1 & 34 & E & 50 & 1049.95 & 0.39 \\
\hline 1100. & 0 & $1 \%$ & $\mathbf{N}$ & 1 & 50 & f: & 50. & 1049.94 & 9.12 \\
\hline 1850. & 0 & $\therefore .3$ & $N$ & 0 & 59 & $\omega$ & So. & 1149.94 & 9.84 \\
\hline $1: 200$. & 0 & 5 & $N$ & 1 & 29 & $w$ & so. & 1199.93 & 10.62 \\
\hline 1250, & 0 & 59 & $\mathbf{N}$ & 1 & 36 & $\mathbf{E}$ & 50. & 1249.92 & 11.44 \\
\hline $1: 00$. & 0 & 57 & $\mathbf{N}$ & 1 & 43 & $\mathbf{W}$ & 50. & 1299.92 & 12.28 \\
\hline 1.350. & $\begin{array}{l}0 \\
n\end{array}$ & 56 & $N$ & 1 & 32 & $\omega$ & $\begin{array}{l}50 . \\
\text { s.n. }\end{array}$ & 1349.91 & 13.09 \\
\hline
\end{tabular}

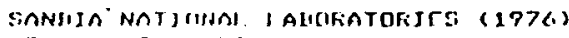

\begin{tabular}{|c|c|c|c|c|c|c|}
\hline 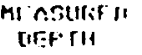 & $\begin{array}{l}\text { Iur: } \\
\text { nint }\end{array}$ & $\begin{array}{l}1 \% \\
115\end{array}$ & & & $\begin{array}{l}\text { ITt } \\
\text { Sto }\end{array}$ & \\
\hline IFT.T & II & $M$ & & I! & $M$ & \\
\hline 1500. & 1 & 1 & $N$ & 0 & 57 & E \\
\hline $1 \leq: 1: 0$. & 1 & ! & $\mathbf{N}$ & 1 & 43 & $\omega$ \\
\hline 1600 & 1 & 1 & $n$ & 3 & 2 & 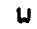 \\
\hline $16: 1 ; 0$. & 1 & 9 & $N$ & 3 & 15 & 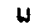 \\
\hline 1200. & 1 & 11 & $N$ & 2 & 5 & $\omega$ \\
\hline $1 \%$. & 1 & 7 & $\mathbf{N}$ & 2 & 5 & 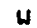 \\
\hline 1000. & 1 & 13 & $\mathbf{N}$ & 0 & 25 & E \\
\hline $111: 0$. & 1 & $1:$ & $\mathbf{N}$ & 6 & 17 & $\omega$ \\
\hline 1900. & 1 & $\because 1$ & $N$ & 4 & 25 & W \\
\hline $1 \because 0$. & 1 & 10 & $\mathbf{N}$ & 3 & 41 & $\omega$ \\
\hline 2000. & 1 & 18 & $\mathbf{N}$ & 0 & 21 & $\omega$ \\
\hline m:o. & 1 & $\because$ & $\mathbf{N}$ & 9 & 22 & $\omega$ \\
\hline 2100 & 1 & $\because 2$ & N & 9 & 9 & $w$ \\
\hline$\because 80$. & 1 & $1:$ & it & 13 & 25 & $\omega$ \\
\hline יoo. & 1 & 19 & N & 11 & 13 & $w$ \\
\hline$\because \cdots, 0$, & I & 11 & II & $\because 3$ & 11 & u. \\
\hline$\because 100$. & 1 & 6 & N & $? ?$ & :5 & $\omega$ \\
\hline$\therefore \therefore 0$, & 1 & 10 & $\mathbf{N}$ & 33 & $\varphi$ & t. \\
\hline :1non, & 1 & 17 & $N$ & 23 & 31 & $\mathbf{w}$ \\
\hline$\because n \because 0$. & 0 & $\therefore 11$ & I & .7 & 3 & 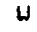 \\
\hline$\because, \infty 0$. & 1 & 0 & $N$ & 90 & 43 & $\mathbf{W}$ \\
\hline 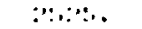 & 1 & () & $N$ & 41 & 31 & W \\
\hline$\because \cdot \because n$, & 1 & 21 & $\mathbf{N}$ & 27 & 4 & $\omega$ \\
\hline$\because . /$. & 1 & $2 \%$ & N & 4.3 & 35 & $\omega$ \\
\hline $2 \therefore 00$. & 1 & $\because 2$ & t & 0 & 30 & \\
\hline 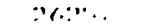 & 1 & 40 & $n$ & 32 & 10 & u \\
\hline 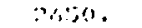 & 1 & $1: 1$ & H & 20 & 13 & 1 \\
\hline 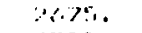 & 1 & 181 & N & $\because 1$ & 3) & 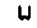 \\
\hline 2700. & 1 & 20 & $M$ & 24 & 1 & \\
\hline
\end{tabular}

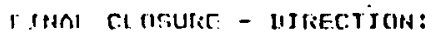
IISTANCE:
W 20 IITG:; 20 MIHS W 10,63 FFET
$\begin{array}{lllllllllll}R & E & C & T & A & N & G & U & L & A & R \\ C & 0 & D & R & D & I & N & A & T & E & S\end{array}$ FEET

\begin{tabular}{|c|c|c|c|c|}
\hline \multicolumn{2}{|l|}{0.00} & \multicolumn{2}{|l|}{0.00} & \multirow{2}{*}{0.00} \\
\hline 0.02 & $\mathbf{S}$ & 0.09 & $\omega$ & \\
\hline 0.02 & $\mathbf{S}$ & 0.20 & $\omega$ & 0.20 \\
\hline $\begin{array}{l}0.01 \\
0.04\end{array}$ & $\begin{array}{l}5 \\
\mathbf{5}\end{array}$ & $\begin{array}{l}0.52 \\
0.81\end{array}$ & $\underset{\omega}{\omega}$ & 0.33 \\
\hline 0.05 & $\mathbf{S}$ & 1.01 & $\boldsymbol{\omega}$ & 0.10 \\
\hline 0.03 & $\mathbf{5}$ & 1.32 & $\vec{\omega}$ & 0.12 \\
\hline 0.04 & $N$ & 1.51 & $\omega$ & 0.21 \\
\hline 0.32 & $\mathrm{~N}$ & 1.72 & $\omega$ & 0.07 \\
\hline 0.82 & $N$ & 1.78 & $\omega$ & 0.27 \\
\hline 1.34 & $\mathbf{N}$ & 1.62 & $\omega$ & 0.23 \\
\hline 1.9 .3 & $N$ & $1.0 ?$ & $\omega$ & 0.1 .5 \\
\hline 2.10 & N & 1.01 & $山$ & 0.1 .5 \\
\hline 3.07 & $N$ & 1.72 & $\omega$ & 0.07 \\
\hline 3.0 .1 & $N$ & 1.78 & $\omega$ & 0.03 \\
\hline 1.26 & $N$ & $1 . H 1$ & IN & 0.18 \\
\hline 4.146 & $N$ & 1.114 & $\omega$ & $0 . \because 0$ \\
\hline 5.48 & $N$ & $1.7 \%$ & $\omega$ & 0.28 \\
\hline 6.17 & $N$ & $1 . \%$ & $\omega$ & 0.14 \\
\hline 6.63 .3 & $N$ & $1.6 ?$ & $\omega$ & 0.11 \\
\hline 7.49 & $N$ & $1 .<0 t$ & $\omega$ & 0.21 \\
\hline B.?2 & $N$ & 1.64 & is & 0.110 \\
\hline 0.97 & $N$ & 1.1 .1 & $\omega$ & 0.14 \\
\hline 9.71 & $\mathbf{N}$ & $1.6: 2$ & $\omega$ & 0.29 \\
\hline 10.50 & $N$ & 1.1 .3 & u & 0.07 \\
\hline $11 \cdot 3.3$ & $\mathbf{N}$ & 1.63 & $\omega$ & 0.17 \\
\hline $12 \cdot 19$ & $\mathbf{N}$ & 1.63 & $\omega$ & 0.13 \\
\hline 13.00 & $\mathbf{N}$ & 1.66 & $w$ & 0.0 .7 \\
\hline דים & 21 & , & ." & $n \cdots$ \\
\hline
\end{tabular}

$$
\begin{aligned}
& \text { CUMFUTATION } \\
& \text { TIME } \\
& \text { TIAIE } \\
& 09: 36: 09 \text { 20-NOU-BY }
\end{aligned}
$$

FAGR: NI. :

TFUE

COIIKSE VIKTICAL. IIEFTH FER T

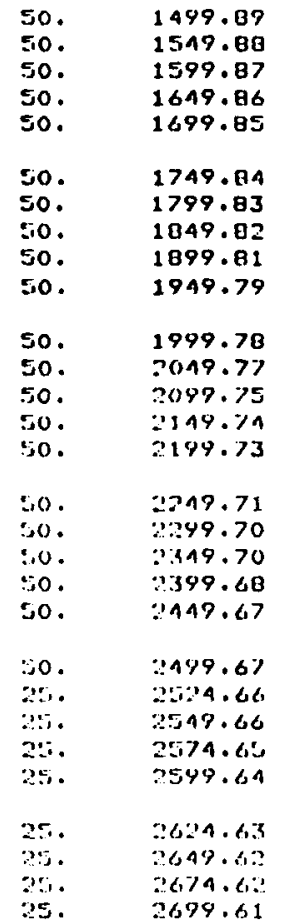

VERTI TCAL FE:T:T

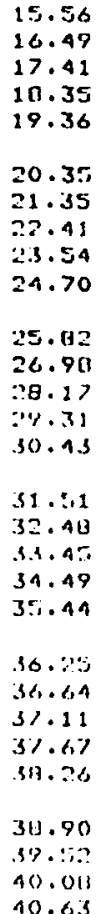

Fi C: T T N G U L A li C D O F II I N A T

\begin{tabular}{|c|c|c|}
\hline 15.53 & $\mathbf{N}$ & 1.37 \\
\hline 16.47 & $\mathbf{N}$ & $1 . t+t$ \\
\hline 17.41 & $N$ & 1.6 .2 \\
\hline 18.35 & N & 1.67 \\
\hline 19.36 & $N$ & 1.72 \\
\hline 20.36 & $\mathbf{N}$ & 1.75 \\
\hline $21.3 B$ & $\mathbf{N}$ & 1.77 \\
\hline 22.45 & H & 1.82 \\
\hline 23.58 & $\mathbf{N}$ & 1.73 \\
\hline 24.74 & $N$ & 2.01 \\
\hline $2 \therefore .67$ & N & $2.0 \%$ \\
\hline$\because 1.03$ & N & $2.1 \mathrm{~S}$ \\
\hline 24,21 & H & 2.31 \\
\hline 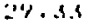 & N & $\because \therefore<$ \\
\hline 30.42 & H & 2.110 \\
\hline 31.96 & $n$ & 3.13 \\
\hline $.32,\{1\}$ & $\mathbf{N}$ & $3.5: ?$ \\
\hline 3.3 .57 & $\mathbf{H}$ & $3 . \%$ \\
\hline $31 .: 27$ & H & 1.34 \\
\hline $3: .16$ & $\mathbf{N}$ & $4.1:$ \\
\hline 45.91 & n & $\therefore .1 \%$ \\
\hline $36 . \therefore<$ & $N$ & $\therefore .10$ \\
\hline $36.6+3$ & in & 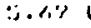 \\
\hline 31.19 & $\mathbf{N}$ & $(1.0) 4$ \\
\hline .57 .24 & $N$ & 6.31 \\
\hline 34.35 & $N$ & 1..5.4 \\
\hline 311.3 .3 & $n$ & 6.03 \\
\hline $34 \cdot 4: 1$ & $N$ & 7.05 \\
\hline
\end{tabular}
FEI:T fillialecis STEVEKITY ris./1001 1

$$
\begin{aligned}
& 0.18 \\
& 0 . \because 4 \\
& 0.21 \\
& \begin{array}{l}
0.27 \\
0.011
\end{array} \\
& 0.13 \\
& 0.22 \\
& 0.30 \\
& 0 . . \\
& 0.15 \\
& 0.41 \\
& 0.01 \\
& \begin{array}{l}
0.210 \\
0.11
\end{array} \\
& 0.80 \\
& 0.1 \text {; } \\
& 0.1 .1 \\
& \text { (1) } 1.5 \\
& 0.0 \% \\
& 0.11 \% \\
& 1.11: \\
& 1.71 \\
& \because \% \\
& \text { 1.117 } \\
& 0.34
\end{aligned}
$$

0.14 


\section{APPENDIX G}

\section{DAIIY REPORTS}

The following pages comprise the daily reports submitted by the mud logger and the reports submitted by sandia field personnel to a distribution list of approximately 75 persons and agencies, including DOE, universities, local government, BLM, and interested scientific investigators. 
Date: 8-02-89 T Time of Feport 08:00 I Days Eince Epud: 0

Dater.-. Heil No. LUF 51-20 i Location: NW 1/4, NE 1/4, Ser.20, T35, R28E, Mone County Co

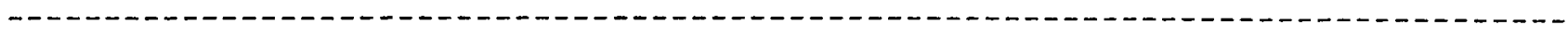
Depth Today 74 : Defth rest. b4: Progrese $10 \mathrm{ft}$ : fotating Hre $1 / 2$

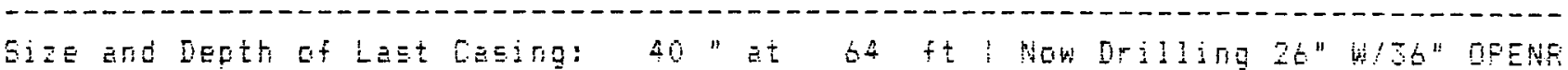

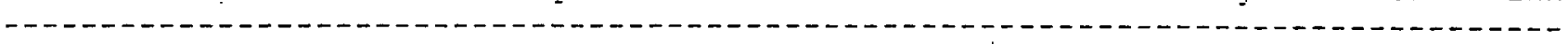
Eit Data:

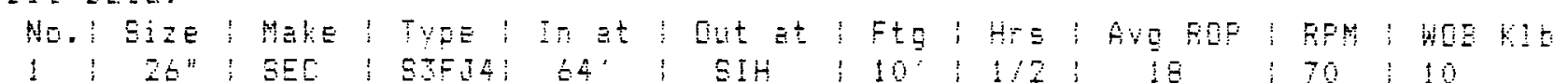

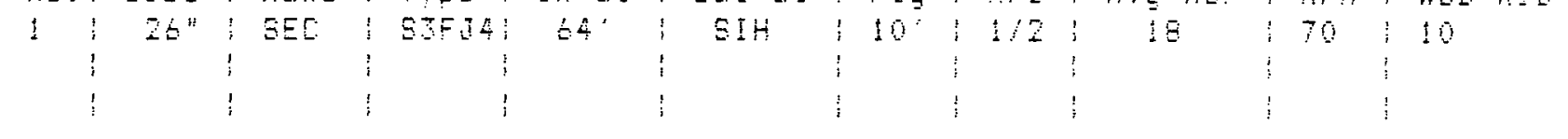

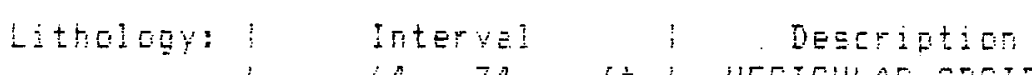

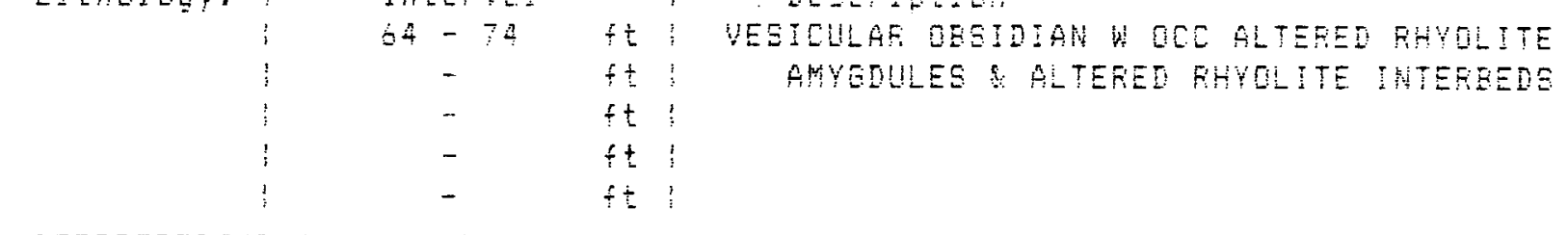

Driling Find:

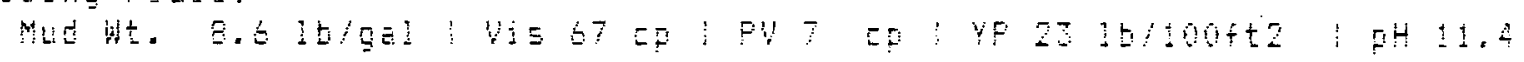

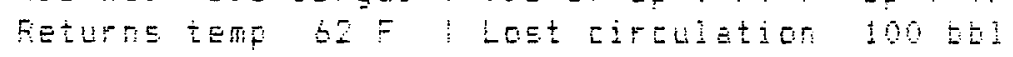

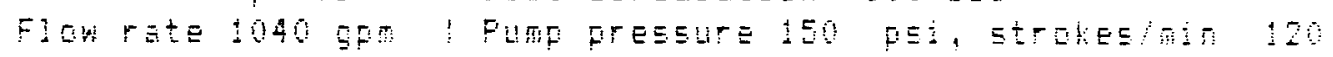

campeition? tentonite, lignite Ottier:

Dril string:

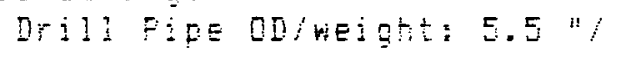

Mumer ef D.F. jeinte:

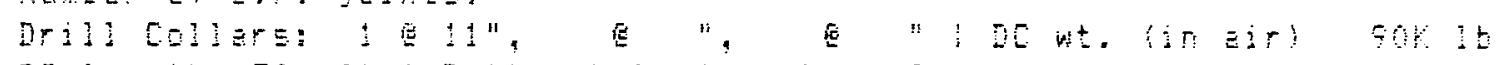

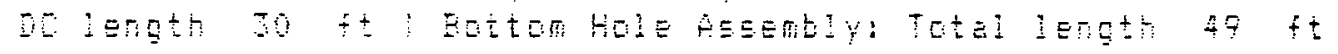

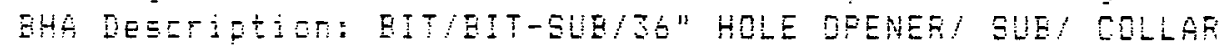

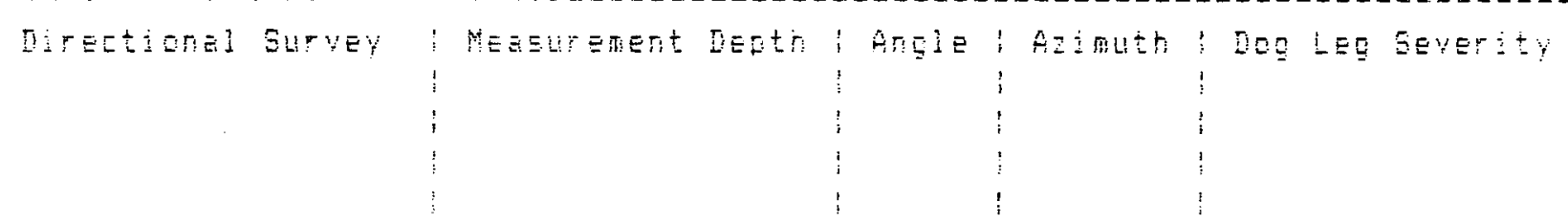

\section{SUAMARY OF YESTERDAYE OFERATIOHS}

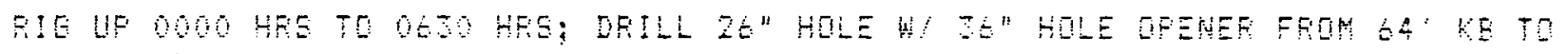
74 ; LOST CIREULATIOH, BUIL MUD VOLUE 


\section{EPOCH WELL LOGGING}

DOE/SANDIA MAGMA ENERGY WELL LVF 51-20

DAILY MUD LOG REPORT

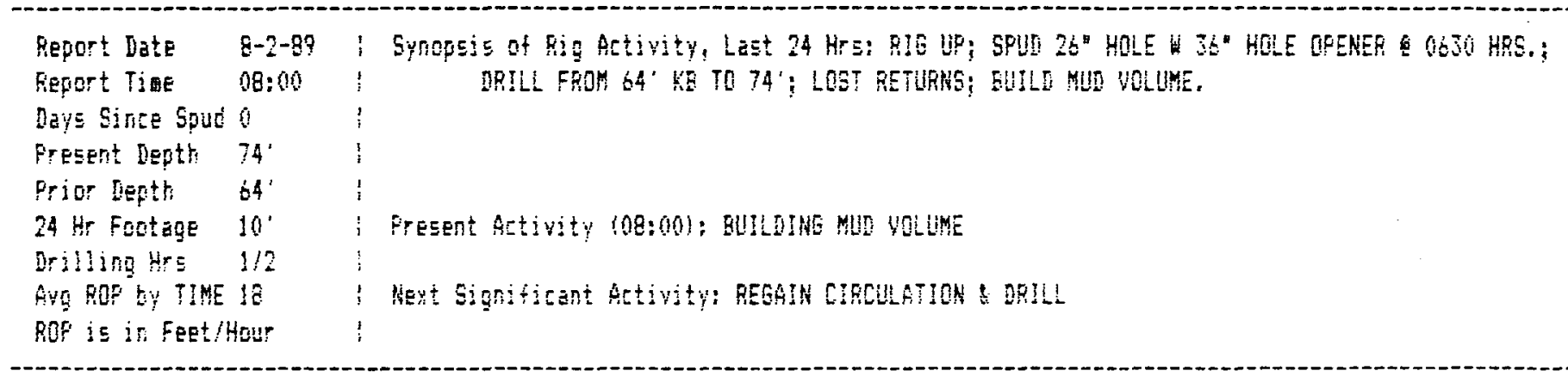

ORILLIME FARAMETERS AHALYSIS

\begin{tabular}{|c|c|c|}
\hline MAU GEFTH & MIN O DEFTH & AUEFAGE \\
\hline 73 & $6 b^{\prime}$ & tY BATE \\
\hline 10 & 10 & $1-1 b=$ \\
\hline 70 & 70 & 70 \\
\hline
\end{tabular}

780 ZAFE

$90 \quad 150 \quad 650 \quad 125 \quad 74^{\circ} \quad 145 \quad p 31$

WUD + GAS PARAMETERE AKALYSIS

\begin{tabular}{|c|c|c|c|c|c|c|}
\hline \multirow[b]{2}{*}{$\mathrm{mo}$} & \multicolumn{2}{|c|}{ WAX I DEPTH } & \multicolumn{2}{|c|}{ HN DEPTH } & \multicolumn{2}{|c|}{ AUESAEE } \\
\hline & 77 & $6 E^{\prime}$ & 26 & $66^{\circ}$ & 5 & pom \\
\hline COND IN & 1010 & $54^{\prime}$ & 992 & 72 & 1000 & pFE \\
\hline Cont & 872 & $84^{\prime}$ & 9077 & $74^{\prime}$ & 894 & PNm $\mathrm{NaCl}$ \\
\hline$H_{2 S}$ & 0 & & 0 & & 0 & pos \\
\hline HDC GAS & 0 & & 0 & & 0 & units \\
\hline$M i$ & 8.8 & & 8.6 & & 8.6 & $1 \mathrm{tet}$ \\
\hline NOWT & 8.6 & & 8,6 & & a.t & $1 \mathrm{bin}$ \\
\hline IN & 11.9 & $70^{\prime}$ & 11.8 & 74 & 11.9 & \\
\hline $01 T$ & 11.8 & $70^{\circ}$ & 11.7 & $74^{\circ}$ & 11.77 & \\
\hline TEMP IN & 65 & $65^{\prime}$ & 84.2 & $74^{\circ}$ & 64.7 & $\operatorname{deg} F$ \\
\hline IEYF OIT & 63.3 & 73 & 63 & 54 & 53.1 & $d \in g^{\circ}$ \\
\hline
\end{tabular}

Con Gas / Trip Gas/ Methane : No Hydrocarbons

CAREIDE LAE Min 6 , unitE, SFH $=$

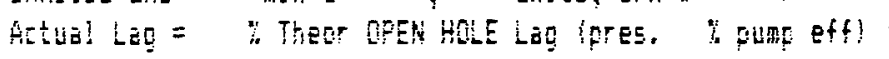
Mu US out = , S00 grase Cartide, Minutes Duration
Theoretical Lag
تiriutes
HOM \#

EIT RECORO

\begin{tabular}{|c|c|c|c|c|c|}
\hline \multicolumn{6}{|c|}{ BIT AECORO } \\
\hline & & & Cin & $\mathrm{CJH}$ & \\
\hline HD. GIZE & MAYETTYFE & IN & FTE & HRS & ConUIIIOH \\
\hline $1 \quad 2 t^{\prime \prime}$ & SEC/5STI4 & $64^{\prime}$ & $10^{\circ}$ & $1 / 2$ & $3 I H$ \\
\hline
\end{tabular}

ITHOSEY

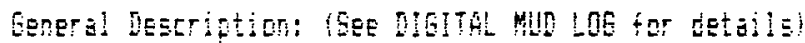

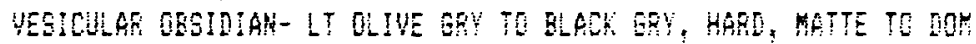
WTHEQUS USTER, COM PERLITIS \& DEVITRIFIED, TFALSUCET TO

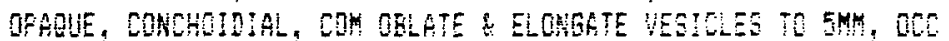
\# WHITE ALTERE RHYOITIC LIUIHGS AND LESE OOH AMYGOULS OF GAME.

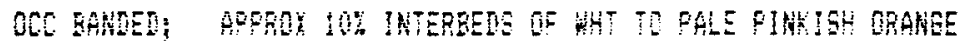
VARIAEL ALIEAED FHVDLITE
Samele Lagong Conditions, other remarks: GOON lOST RETURS $74^{\circ}$, SMMLES FEOM $71:-74$ GTILL IN HOLE.
Sande Shater Stren SIzE: $\frac{H}{\|}=40 / 60$ \& $\# 2=40 / 60 \mathrm{MESh}$ Latest Srreen Size Charge on: 6-2-g9 (Start of well) 


\section{DOE / GANDIA MAEMA ENEREY EXPLORATORY WELL}

Date: 8-03-89 i, Time of Report 08:00: Days since spud: 1

Wel1 No. LVF $51-20$ : Location: NW 1/4, NE 1/4, Sec.20, T5S, R2BE, Mono County CA

(Depth Today

Depth Today $91^{\prime}$ ' Depth Yest. 74 ' Progress $17 \mathrm{ft}$ I Rotating Hrs 3.5

Size and Depth of Last Casing: 40 "at bj ft i Now Drilling 26"/36" Hole

gize and Depth of Last Casing:

Bit Date:

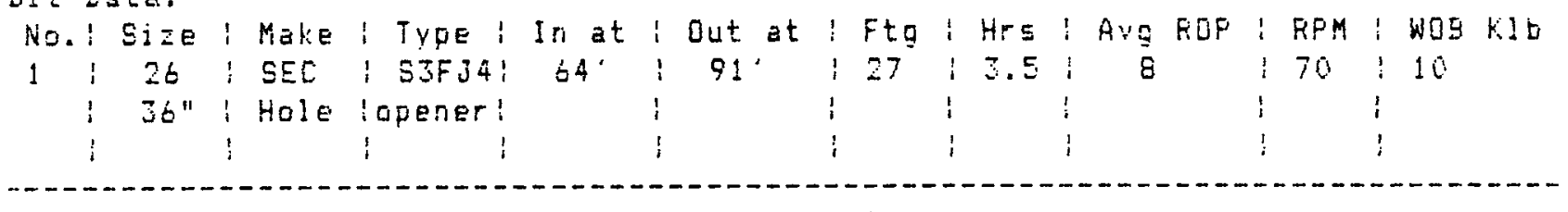

Sithology: : Interval ft: cost CIRCULATION, NO RETURNS TO SUFFACE

- $\quad$ ft: LOST CIRCULATION. NO RETURNS TO SURFACE

- ft i

$-\quad+t \quad$ i

- $\quad f t \quad i$

Jrilijine Filuid:

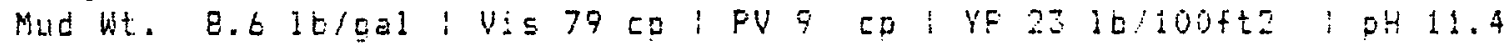

Feturne temo t4 F l Lost circulation 1200 tb:

Flow rate 1040 gom : Fump pressure 145 osi, strokes/miri 120

Complestion: bentonite, ígnite

ottier: Iine, caustic soda

jo il string:

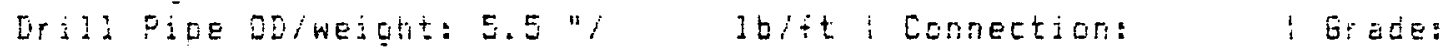

Number of D.F. joints:

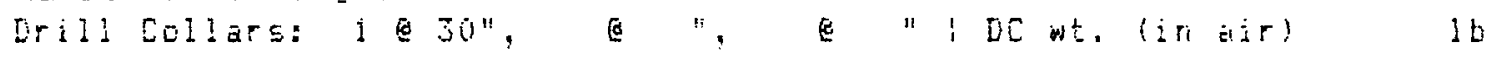

DC iength $30 \mathrm{ft}$ : Bottom Hole Assemily: Total lenoth $49 \mathrm{ft}$

BHA DESCriotion: BIT/EIT SUE/30" HOLE OFENER/SUE/COLLAF

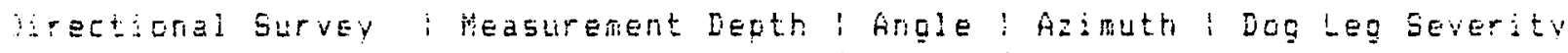

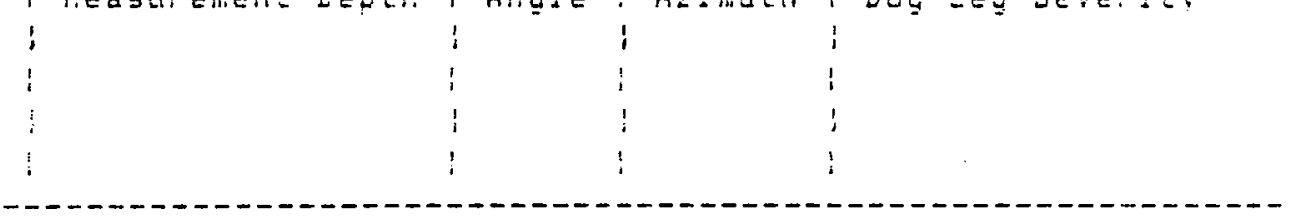

SUMMARY QF YESTERDAY'E OFERATIONE

EUILO MUD VOLUME, J.5 HFS REFAIFING SHAKEFS. DFILL FFON 74'-9!' BUILD MUD VOLUME, LAY DOWH BHA. RIG LIF \& CEMENT 202 FT3 CEMENT FLUG, HAIT DH CEMENT: CEMENT FLUS 12 202 FTS, WOC, CEMENT FLUG \#3 310 FT3. CEMENT W FEFLITE. WOC

EEFOR LY: JOHN FINEEF 


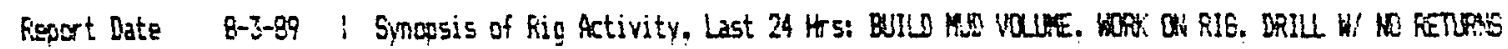

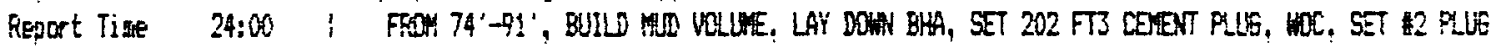

- Days Since Spido : Fresent leath $9:$. Price Depti $74^{\circ}$ 24 Hootage 17 Mrilling trs 3 Avg Gite by TIE 6 ROF is in Feet/Hour

\author{
1 QF 202 FTS, WX, SET 33 PLUG OF 310 FT 2-1 FEFLITE, WXS
}

$i$

Fresent Activity 108:00): HA!TING ON CEXERT

Ment Significant Rativity: TEST WUE

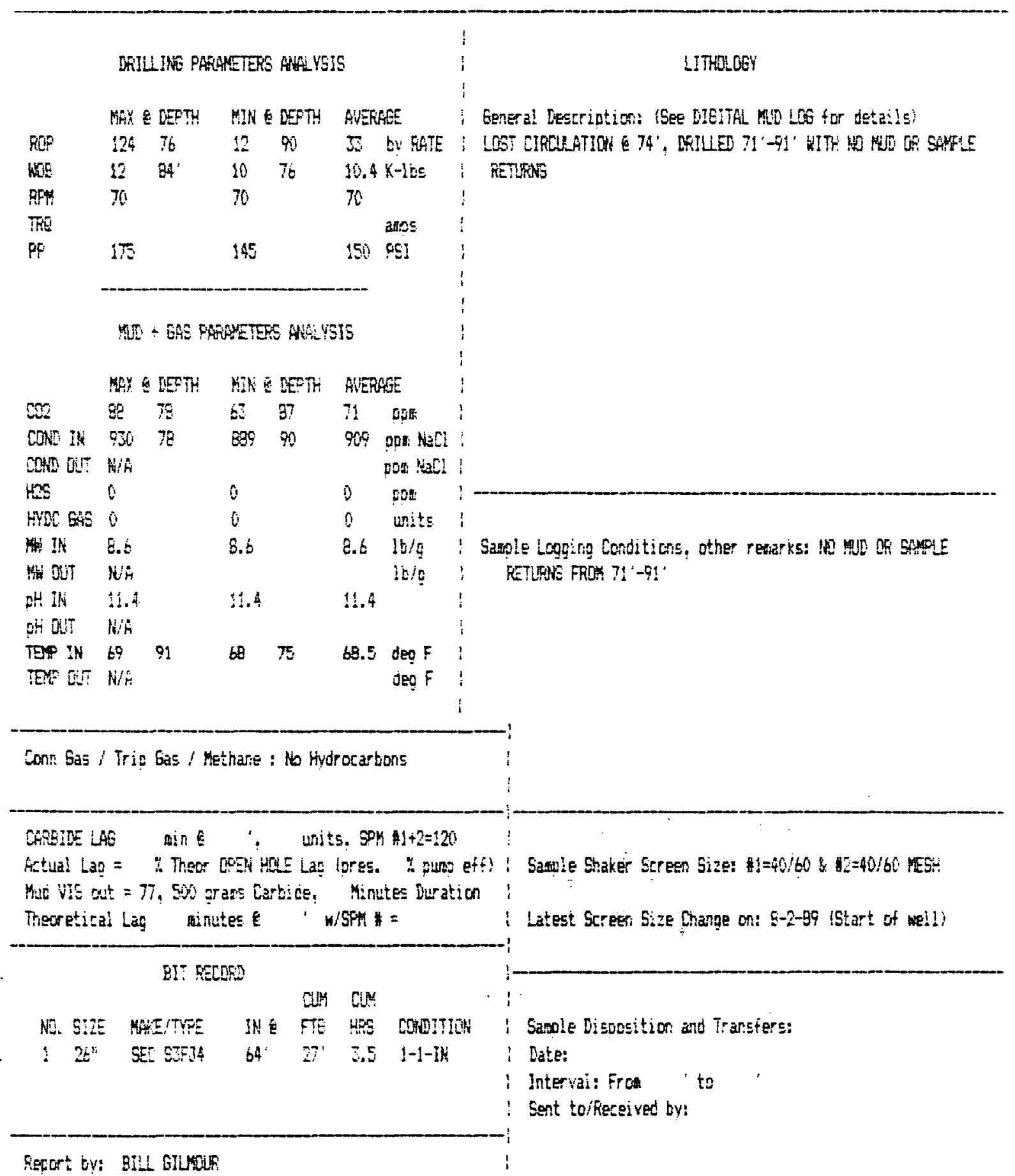


Date: 8-04-89; Time of Report 08:00; Days since 5pud: 2

Wel1 No. LVF $51-20$ i Location: NW 1/4, NE 1/4, Sec.20, TJS, F28E, Mono County CA -

Depth Today 96'' 'Depth Yest., 9!'' 'Progrese 5 ft ' Rotating Hrs 2.5

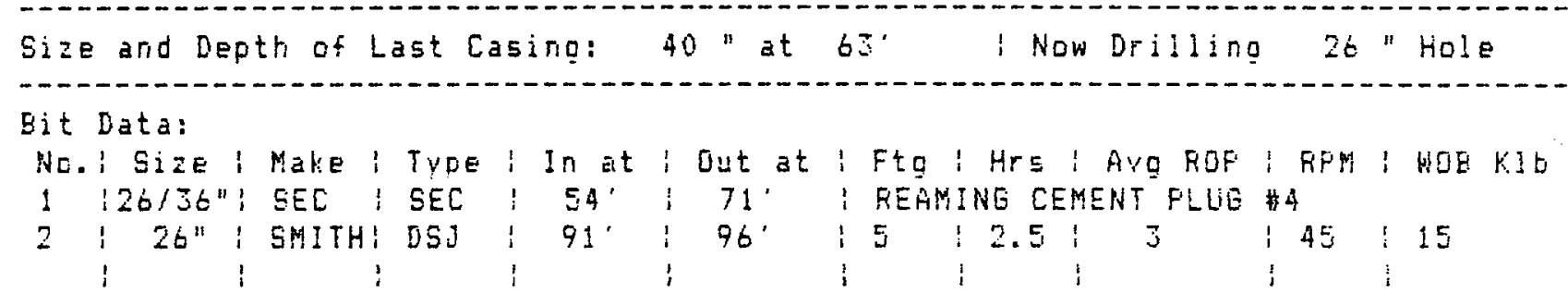

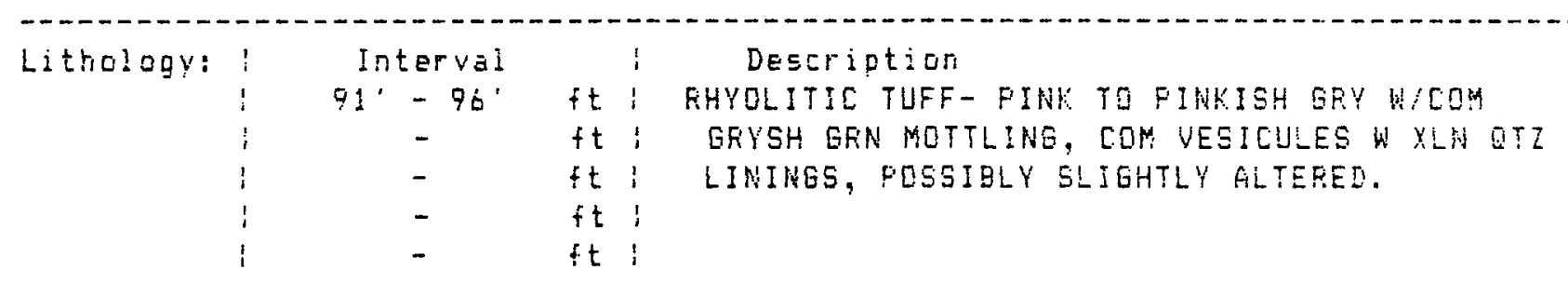

Drilling Fluid:

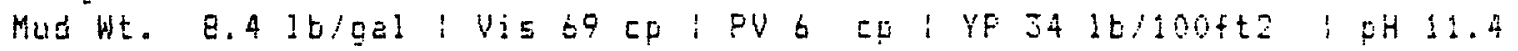

Feturns temp bj F I Lost circulation 900 bbl

Flon rate 507 gpa i Fumperessure 55 pei, strolesidir 59

Composition: bentonite. LIME, CAUETIL SODA

nttier:

Drili string:

Drij Fipe obivejetit: $5.51 /$

lblft i Conrection: i Grede:

Number of D.F. iojints:

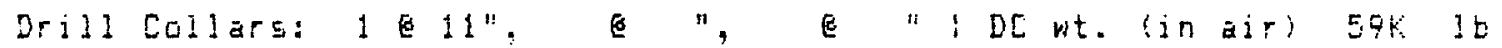

DE length $30 \mathrm{ft}$ : Eottom Hole Asembly: Total length $49 \mathrm{ft}$

BHA DEEETIDEIOR: BITIEIT SUEIHOLE OPENER/SUS/COLLAR

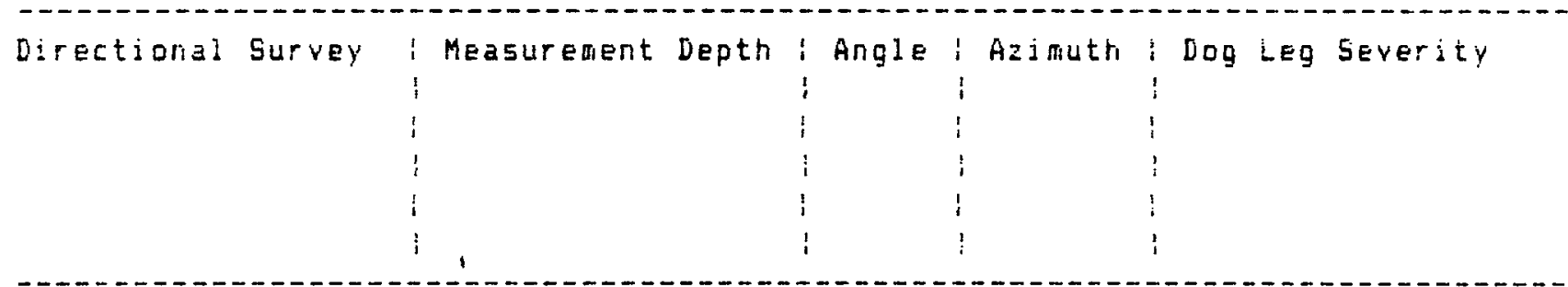

SUMMAFY OF YESTEFDAY'S DFERATIDNS

WAIT ON CEMENT, PUMF 10 SKS EEL \& EMPTY MUO SACKS AS LCM, CIFE HOLE CLEAN. FIU SO" HOLE OFENER. DRILL FROM 9!'-95' LOST FETUFNS. BU!LD MUD VOLUME. OFILL TO 9E, LOET CIRCULATION, CIRCULATE, POOH FOR CENENT FLUG \#4, CEMENT W JIO FTJ 2-1 PERLITE, WAIT ON CEMENT. FIH TAG CEMENT E $54^{\circ}$ :P/U $36^{\prime \prime}$ HOLE DFENES. DRILL SOFT CEMENT TO 71\%. CIRSULATE.

REport by: JOHN FINGEF 
EPOCH LOESING

DOE/SANDIA MAGMA ENERGY WELL LUF 51-20

DAILY MUD LOG REPORT

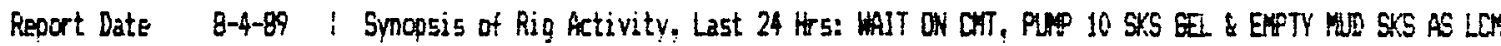

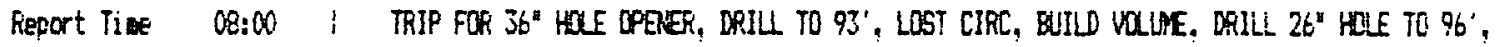

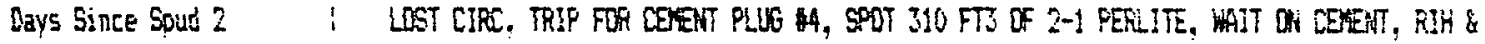

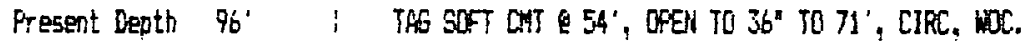

Friar Depth 9.'

24 Hrotage 5

Drilling Hrs 2.5

AvQ ROF by TINE 3

(:Tesent Activity (08:00): CiRC/WCC

Prof is in Feet/hour

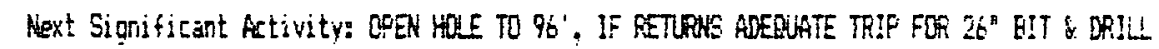

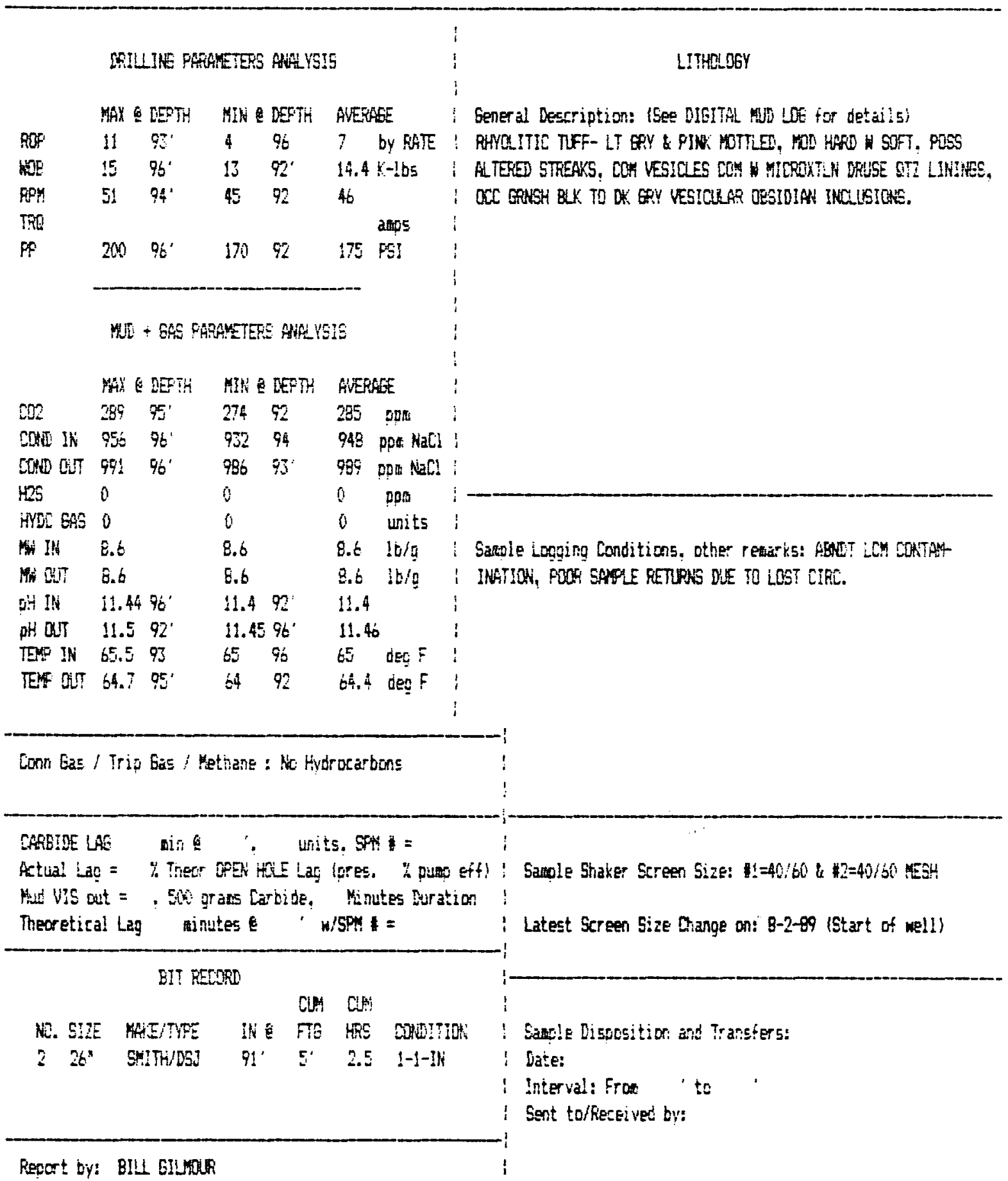


ite: 8-05-89: Time of fieport 00:00: Days since spud: $:$

I1 No. LVF $51-20$ i Location: NW 1/4, NE 1/4, SEC.20, T3S, F28E, Mono County CA epth Today 143 ', Depth Yest. 96, i Progrese 47 ft i Fotating Hrs 4.5 ze and Depth of Last Casing: 40" at bJ'Ke I Now briling 26 "Hie $t$ Data:

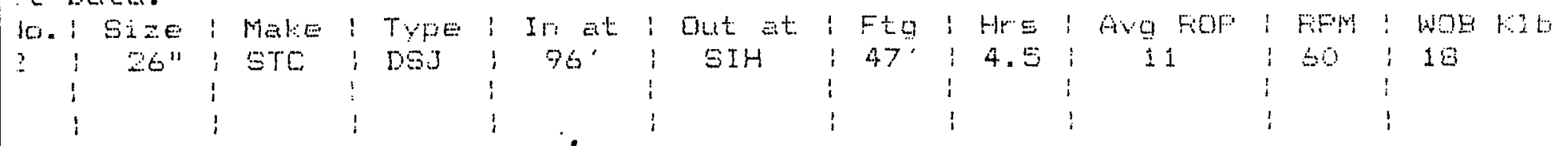
thoiogy: Interval i Description

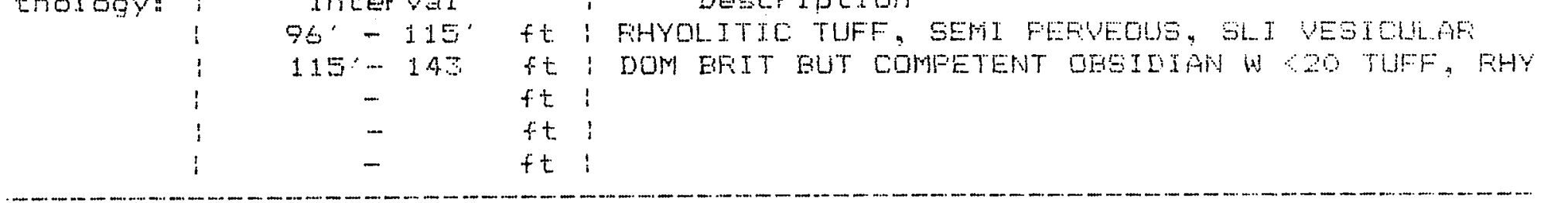

ili ing Fluida

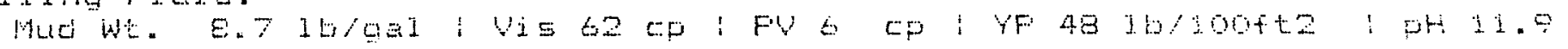

Feturne temp $72 \mathrm{~F}$ i Lost circulation gen be?

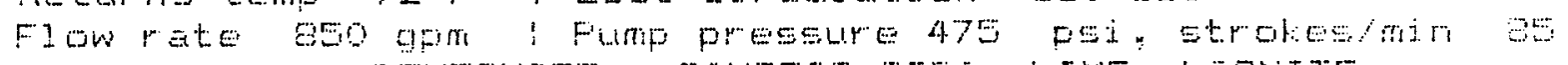

Compostion: EENTONITE * CAUSTIE SODA. LIME, LIGNTTE Detrer:

iil $\operatorname{string:}$

Drj.j. Fipe oD/weight: . $_{5}$ "'

le/ft : Eorrection:

Srace:

Number of D.F. joirits:

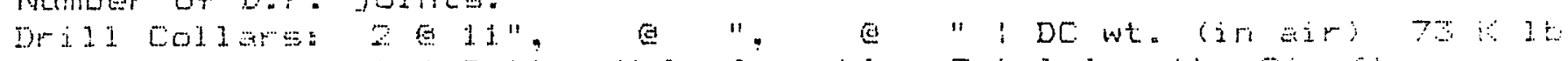

Do length bo ft $\quad$ Eottom Hole Assembly Total lencth 90 ft

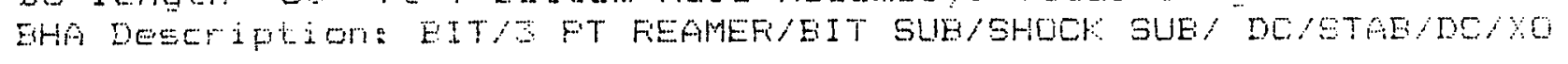

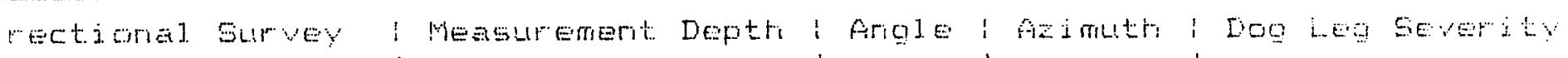

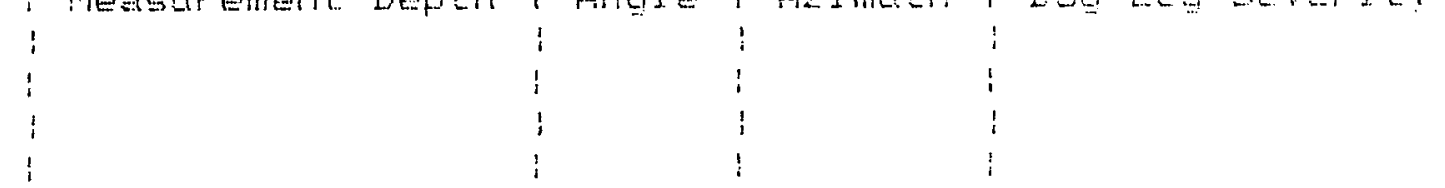

SUMMAFY OF YESTEFIDAY'S DFEFATIQNS

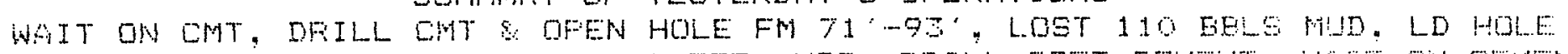
OFENEF, FU S ITE DF, CEMENT 310 FTE, WOC, DFILL SOFT CEMENT, WAIT ON CEMENT, DEILL HAFD CEMENT $79^{\circ}-94^{\circ}$, CHANGE BHA, DFILL FFOM $96^{\circ}-14 \mathrm{~S}^{\circ}$ 
EPOCH MELL LOSGING

\begin{tabular}{|c|c|c|c|}
\hline Report Date & $8-5-89$ & 1 & Synopsis of Rig Activity, Lest $24 \mathrm{Hr}$ : HA!T ON CEMENT, DRL CHT \& OFEH HULE TO $93 .$, LOST 110 \\
\hline Feport Tine & $08: 00$ & $i$ & BBLE, LAY DOHN HOLE DPENER, F/J 3 JTE DP, PUMF CEKENT PLUE E5- 310 FT? 2-1 PERLITE, WOC, \\
\hline DEys Sirice Spud & 3 & ! & DRILL SOFT OMT TO 78', EIRC CMT, WOC 4 HRE, DEILL CEMENT TO 94', PQOH LAY DOHWH HDLE OPENEE \\
\hline Fresent Depth & $143^{\circ}$ & $i$ & RIH \& DEILL $26 "$ HOLE TO $143^{\circ}$ \\
\hline Prior Deth & $96^{\circ}$ & i & \\
\hline 24 Hr Footage & 47 & $i$ & Present Activity $008:(0)$ : DRILLIHS \\
\hline Drilling Hrs & 4.5 & 3 & \\
\hline Avg BOE by TIME & $\dddot{1}$ & $i$ & Ment Significant Activitu! DRILL \\
\hline ROP is in Feet & & i & \\
\hline
\end{tabular}

DRILLING PARAKETERS ANHLYSIS

\begin{tabular}{|c|c|c|c|c|c|c|}
\hline & HAX & DEPTH & MIN & DEFTH & \multicolumn{2}{|c|}{ AVERTAGE } \\
\hline ROP & 102 & 122 & 1 & $114^{\circ}$ & 17 & by RATE \\
\hline HeE & 20 & $115^{\prime}$ & 10 & $109^{\circ}$ & $: 5$ & $k-135$ \\
\hline RPH & $b i$ & 116 & 47 & $98^{\circ}$ & 55 & \\
\hline TRQ & & & & & & ands \\
\hline$p ?$ & 475 & $116^{\circ}$ & 370 & $\$ 14^{\circ}$ & 455 & FS: \\
\hline
\end{tabular}

MUD + GAS FARANETERS HWHLYEIS

\begin{tabular}{|c|c|c|c|c|c|c|}
\hline & MAY & DEFTH & E:N & DEFTH & AVER & $A G E$ \\
\hline $\mathrm{Cog}$ & $46 !$ & $110^{\circ}$ & $339 !$ & 97 & 389 & ppes \\
\hline cono IN & 3291 & $108^{\circ}$ & 2555 & $142^{\prime}$ & 2667 & pos: $\mathrm{KaC}]$ \\
\hline COHE EUT: & 295 & 122 & 2690 & $107^{\circ}$ & 2754 & Deta MaS \\
\hline 425 & 0 & & 0 & & 0 & pos: \\
\hline WYAS SAS & 0 & & 0 & & 0 & units \\
\hline 制 & 8.7 & & 8.7 & & 8.7 & $15 / 9$ \\
\hline Mis OUT & 9.7 & & 8.7 & & 8.7 & $16 / 0$ \\
\hline $\mathrm{FH}$ IN & 12.0 & $98^{\circ}$ & 11.9 & $143^{\circ}$ & 11.9 & \\
\hline Oن & II.: & $57^{\circ}$ & 11.7 & 145 & 11.9 & \\
\hline TEMF IN & 77 & $142^{\circ}$ & 65 & $97^{\circ}$ & 72 & deg F \\
\hline TEHE OUT & 78 & $143^{\circ}$ & 70 & $97^{\circ}$ & 73 & $\operatorname{deg} F$ \\
\hline
\end{tabular}

\section{LITHOLGY}

Genera! Descriation: (See Misitai MUD lob for detej!s) RHYOLITE-GENSH GRY ORHESH FNK TUFFACEOUS AKYGDULES AMO LAKS,

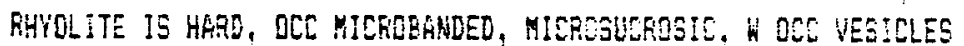
LINES \& OCC FILLED TUFF, OCC CARBOHACEOUE JWCLUSIOHS, DVEFGL HOD COMPETELT EXCEPT FOR TUFFACEOUS MATERIAL WHICH IIOPLGYS PAPTIAL OISSOLUTIOH, ABNOT CEDER FISER AONER AE LUET CIRCUSATIOH MATERIAL.

\section{Conn Eas / Trip Gas / Methane : No Hudrocarbons}

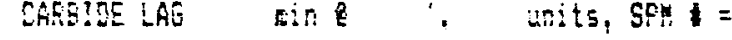

Actual Laq = "Theor OPEN Héc Lag (pres. I ouap eff) Hud Vis out $=$, 500 crats Carbide! Minutes Duration

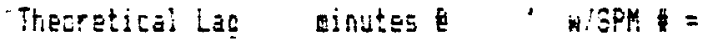
B!T FECORS

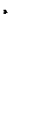


Date: 8-06-89 I Time of Feport 08noo! Days since spud: 4

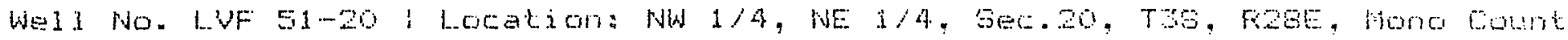

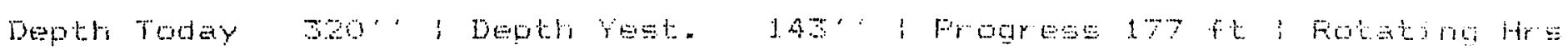

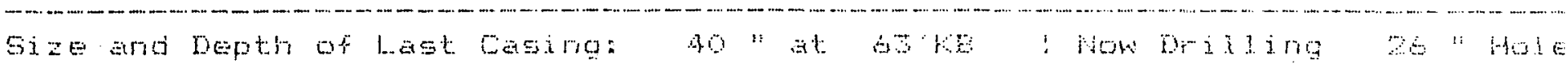
G

Eit Datan

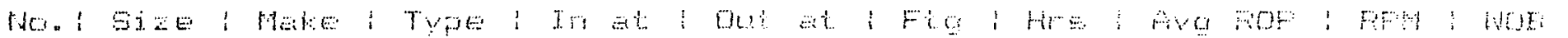

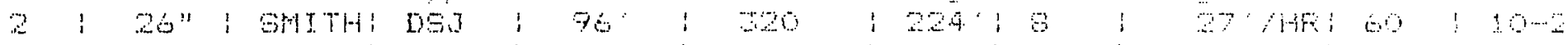
$\begin{array}{ccccc}1 & \vdots & \vdots & \vdots & \vdots \\ \vdots & \vdots & \vdots & \vdots & \vdots\end{array}$

\begin{tabular}{|c|c|c|c|c|c|}
\hline \multirow[t]{6}{*}{ L ithology: } & ! & $\operatorname{Int} E r+1$ & & : & 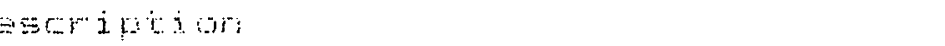 \\
\hline & $\mathfrak{i}$ & 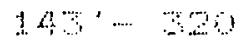 & $r t$ & 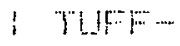 & 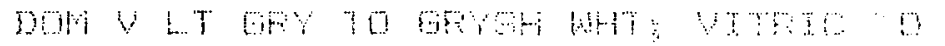 \\
\hline & ; & $\ldots$ & $\because+$ & 1 & 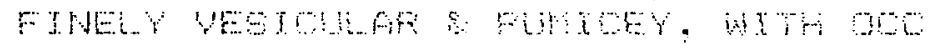 \\
\hline & $i$ & - & $+t$ & 1 & 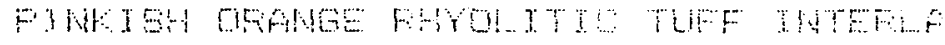 \\
\hline & 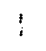 & $\cdots$ & $r$ & $i$ & 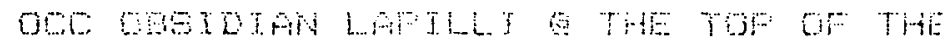 \\
\hline & i & $\ldots$ & $\because \mathrm{t}$ & $i$ & M1TEFVमL. \\
\hline
\end{tabular}

Drilling Fluid:

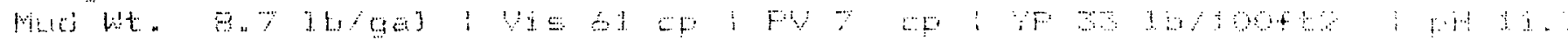

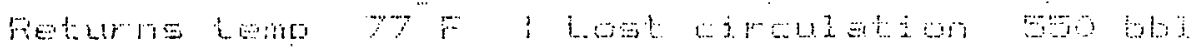

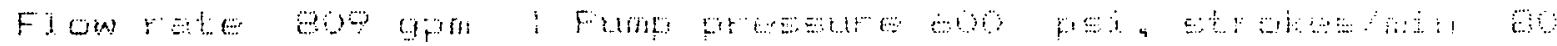

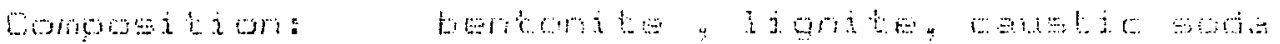

thin:

Dria etring:

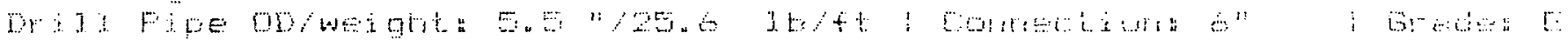

Wumber of D.F. joint: 4

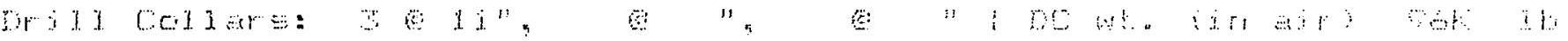

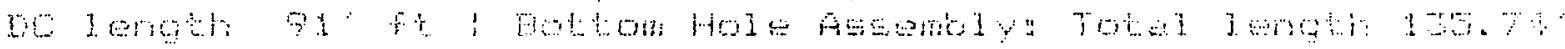

MHF besm

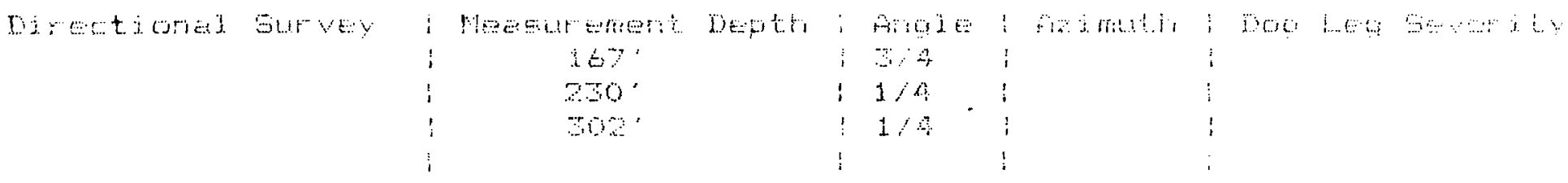

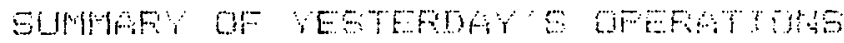

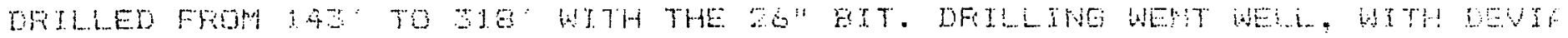

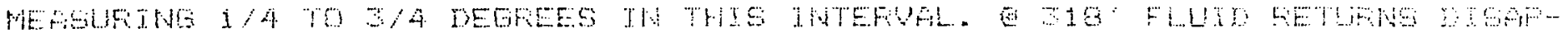
FEAFED COMNLETELY, SO ANOTHEF CEMENT PLUG WAS SET AT THE EOTTOH OF THE HOL

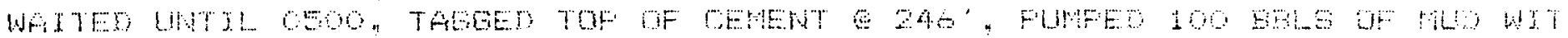

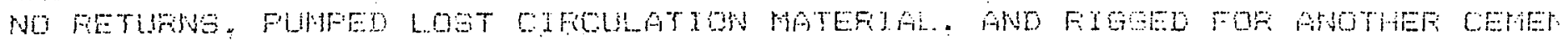
Fing....

Fieport by: JOHN FINGEF: 


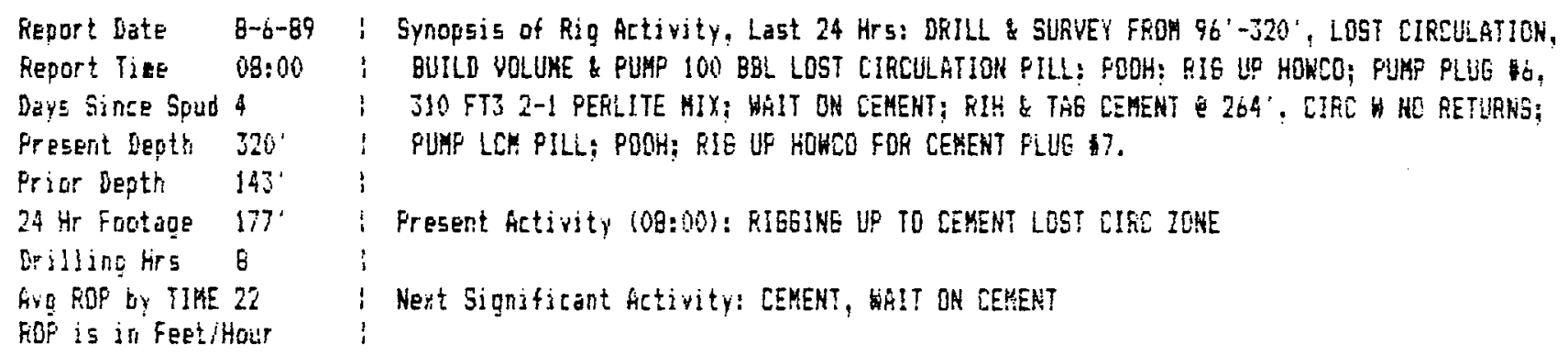

DRILLING PARAMETERS ANALYSIS

\begin{tabular}{|c|c|c|c|c|c|}
\hline MÁ & DEFTH & MIN & IEPTH & \multicolumn{2}{|c|}{ ANVEAGE } \\
\hline 80 & $167^{\prime}$ & 4 & 310 & 34 & by RATE \\
\hline 28 & $245^{\circ}$ & 7 & $174^{\circ}$ & 13 & $k-1 b 5$ \\
\hline 75 & 275 & 50 & $289^{\circ}$ & 56 & \\
\hline 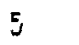 & $244^{\circ}$ & 3 & $289^{\circ}$ & 3 & asps \\
\hline 1000 & 215 & 438 & 278 & 719 & PS \\
\hline
\end{tabular}

MUO + GAS PARANETERE RNGLUSTS

\begin{tabular}{|c|c|c|c|c|c|c|}
\hline \multirow[b]{2}{*}{$C 02$} & \multicolumn{2}{|c|}{ HAX E DEFTH } & \multicolumn{2}{|c|}{ MIN E DETTH } & \multicolumn{2}{|c|}{ AVERAGE } \\
\hline & 65.4 & $258^{\prime}$ & 355 & 180 & 457 & 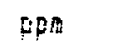 \\
\hline Wi is & 2837 & 143 & 1375 & 310 & 2330 & pos HaC! \\
\hline CUAS OLIT & 2772 & $182^{\prime}$ & 1268 & $304^{\circ}$ & 1889 & pto: $\mathrm{KaCl}$ \\
\hline 925 & 0 & & 0 & & 0 & ppar \\
\hline HWC SAE & 0 & & a & & 0 & units \\
\hline WiN & 8.8 & $200^{\circ}$ & 0.7 & $309^{\prime}$ & 8.8 & $15 / 0$ \\
\hline OLST & 6.9 & 187 & $8 . ?$ & $313^{\prime}$ & 8.8 & $\mathrm{lb} / \mathrm{g}$ \\
\hline Ili & 11.9 & $144^{\prime}$ & 11.6 & $320^{\prime}$ & 11.7 & \\
\hline $00 \mathrm{TT}$ & 11.9 & $144^{\circ}$ & 11.6 & $311^{\circ}$ & 11.8 & \\
\hline TEHE IH & $8^{5}$ & $320^{\circ}$ & 77 & $145^{\prime}$ & 81 & deg F \\
\hline EHF 036 & 87 & 318 & 78 & $145^{\prime}$ & 22 & dec $F$ \\
\hline
\end{tabular}

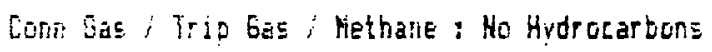

\section{LTHOLS6!}

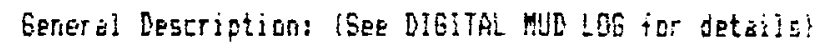
DOS VITRIC TUFF- UT TO IT GRY, OCL NED EPY! PE TO MOD WELRED; FIFW TO HOD KARD: HATTE TO SUEVITREOUS; OCE OEUTTHIFIEL; COM TO $\checkmark$ ABNDT ELONGATE TRARSLUCERT GLAES SHARDS \& FRAES: COF VESICLES GRADINS TO FLYICE: TF TO DCE PIHES RYHILITIC TUFF. TRACES LAPILLI TUFF $150^{\circ}$ WITH OCE SEkNO OH GRY GESIDIAK LAFILL1.

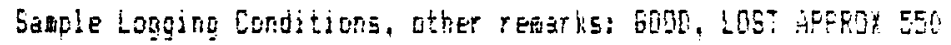

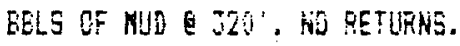

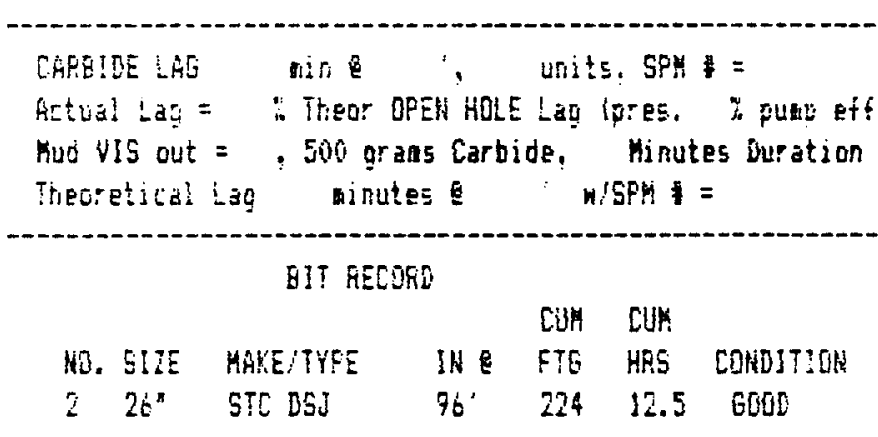

Saple Shaker Sereen Size: $1=40160$ \& $2=40 / 60$ MESH Latest Screen Size Ellange orta 8-2-89 (Start of well) $-1$ 
Date: 8-07-67 : Time of Feport 08:00 i Days since spud: 5

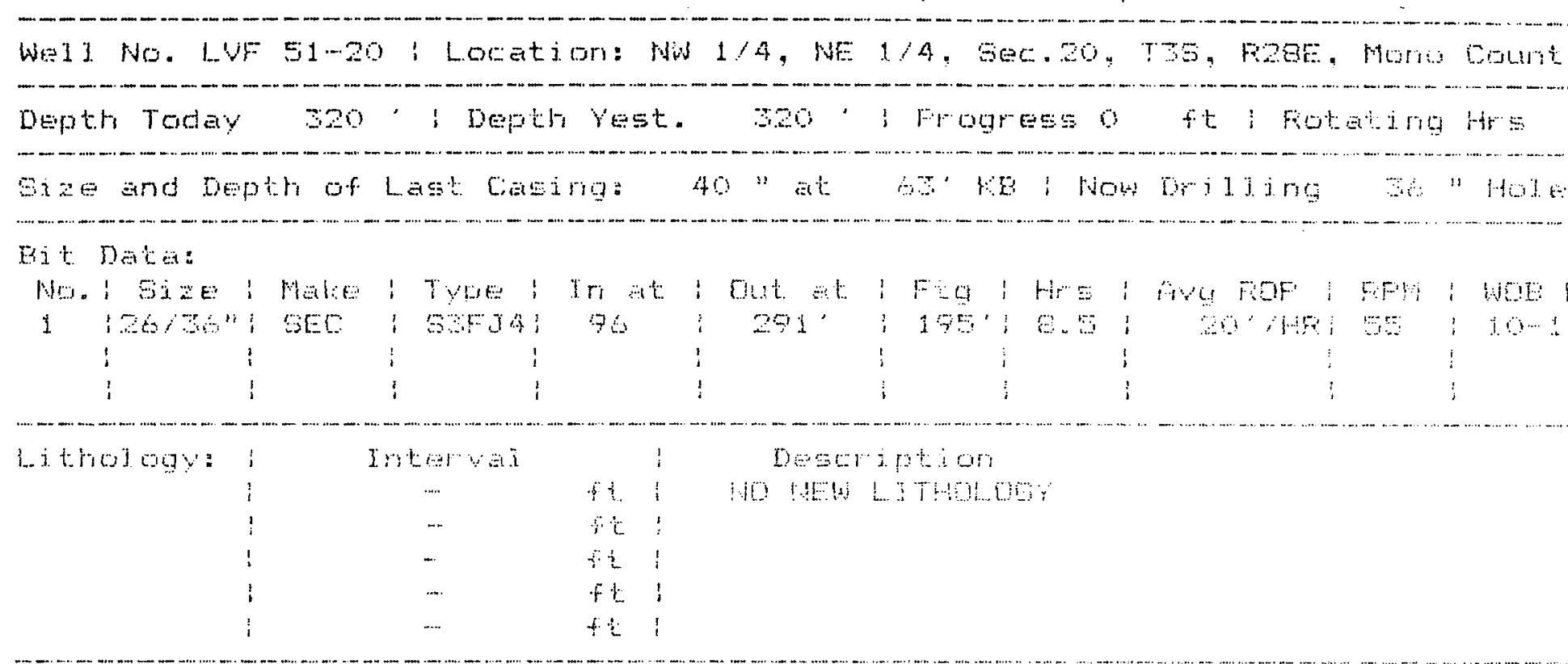

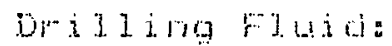

wath. 6.

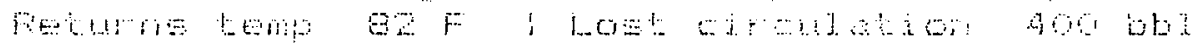

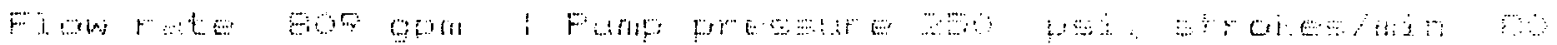

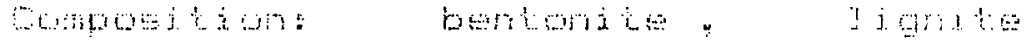

ation:

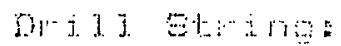

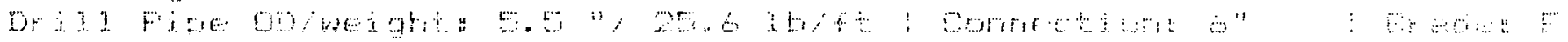

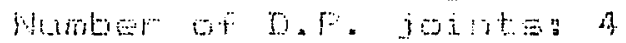

Wil

Th

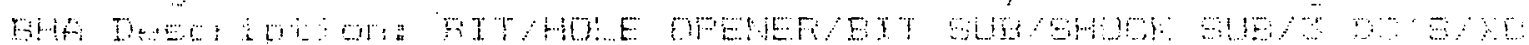

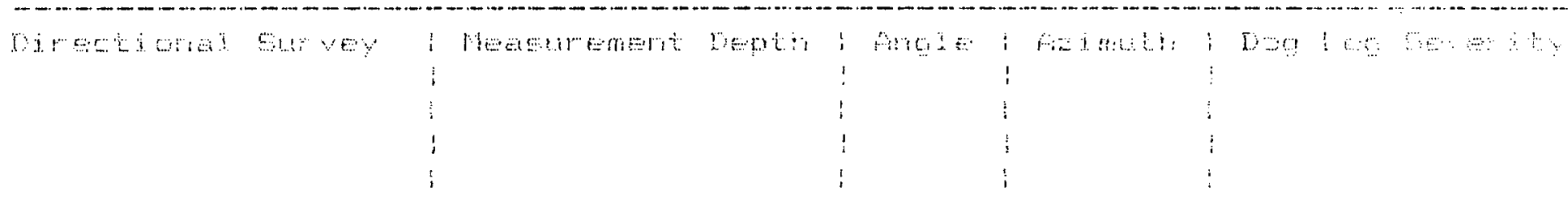

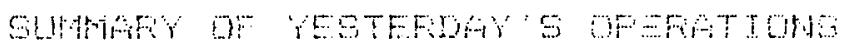

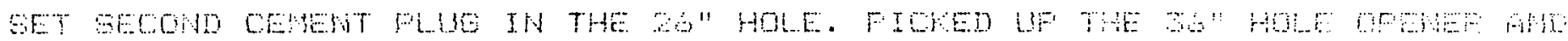
YFTLED TO

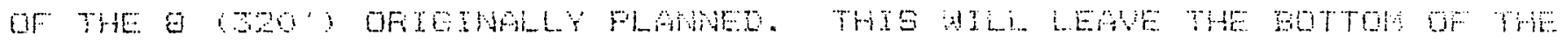

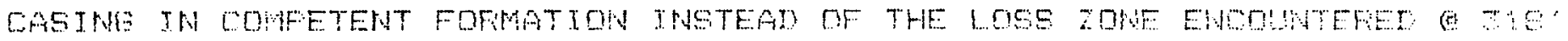

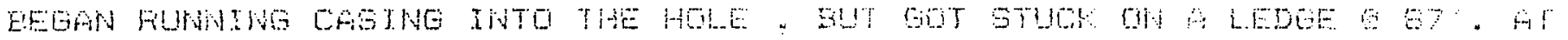

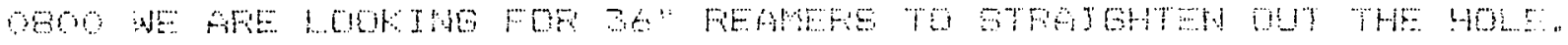




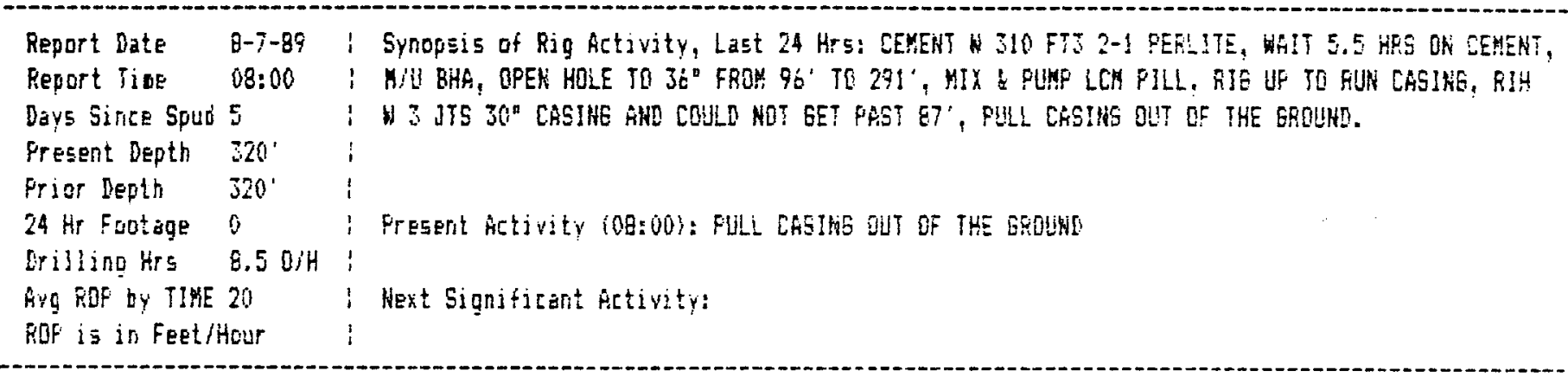

DRILLING PAEAMETERS ANRLYSIS

FOF

HDB

FFM

ing

PF

\section{ANT GEFTH}

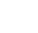




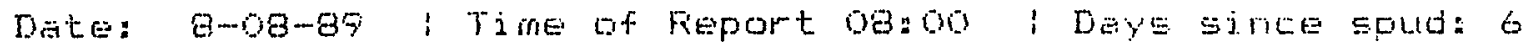

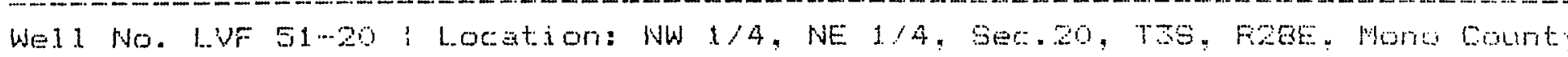

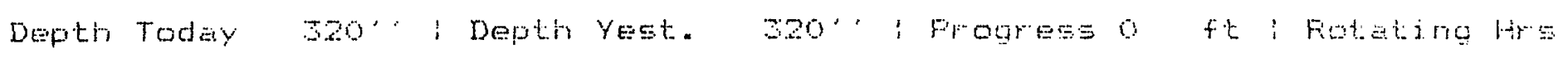
Size and Depth of tast Casing: 40 " at b.

Bit Date:

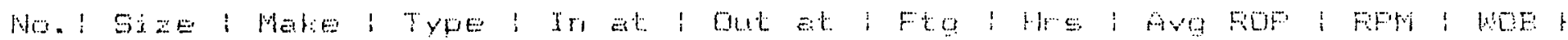

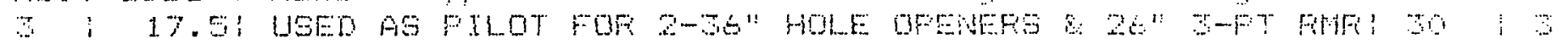
$\begin{array}{cccc}1 & 1 & \vdots & \vdots \\ 1 & i & i & \vdots\end{array}$

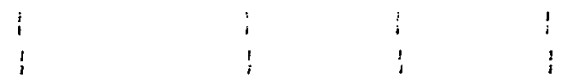

\begin{tabular}{|c|c|c|c|c|c|c|}
\hline \multirow{6}{*}{ Litrodogy } & $i$ & Intersal & & $i$ & & Descor jot \\
\hline & $i$ & $\cdots$ & $\vec{t} t$ & $\vdots$ & & \\
\hline & $!$ & $\ldots$ & $f i$ & $i$ & $\mathrm{NO}$ & NEW L T THOLGO' \\
\hline & 1 & $\ldots$. & $f t$ & 1 & & \\
\hline & i & $\cdots$ & $r t$ & : & & \\
\hline & i & $\cdots$ & $f t$ & 1 & & \\
\hline
\end{tabular}

Dilizing Fluds

Whe we E.

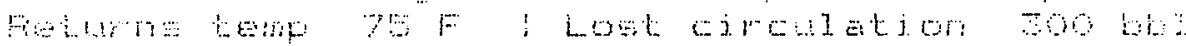

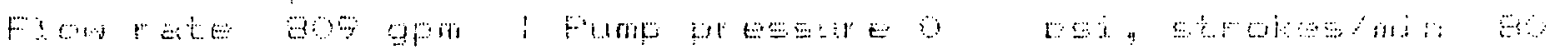

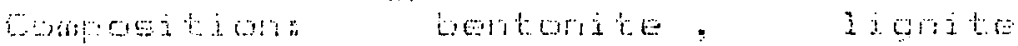

a.t: : : :

Wri.

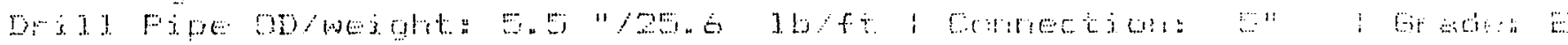
number of D. P. jonts: o

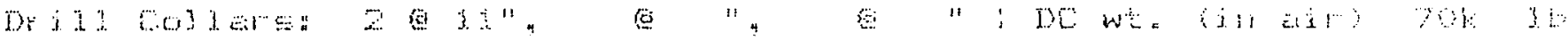

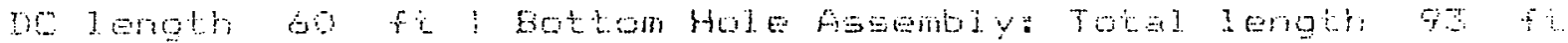

DHA WE

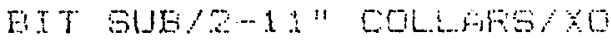

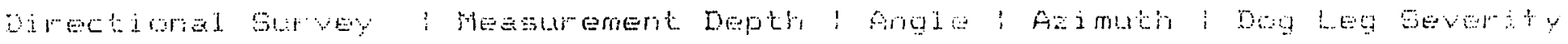

$\begin{array}{cccc}\vdots & \vdots & \vdots \\ \vdots & \vdots & \vdots \\ & \vdots & \vdots & \vdots\end{array}$

SUNATAFY OF YESTEROAY S OFEFATIONO

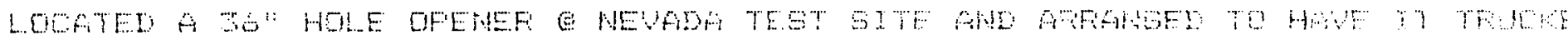

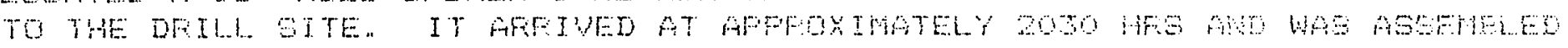

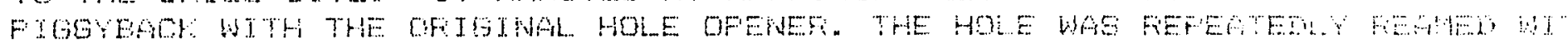

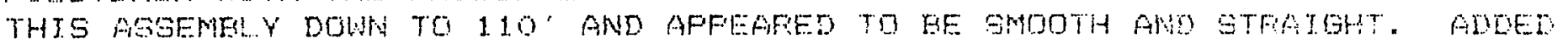

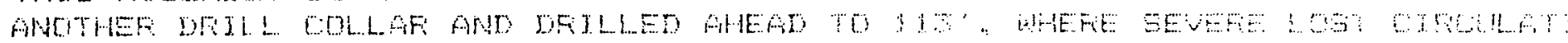

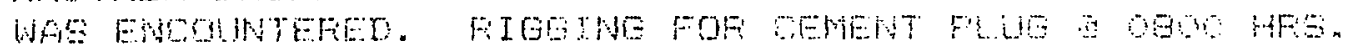

Feport by: JoH FTMEEF 


EPOCH MELL LOSGING
DOE/SANDIA MAGMA ENERGY WELL LVF $51-20$

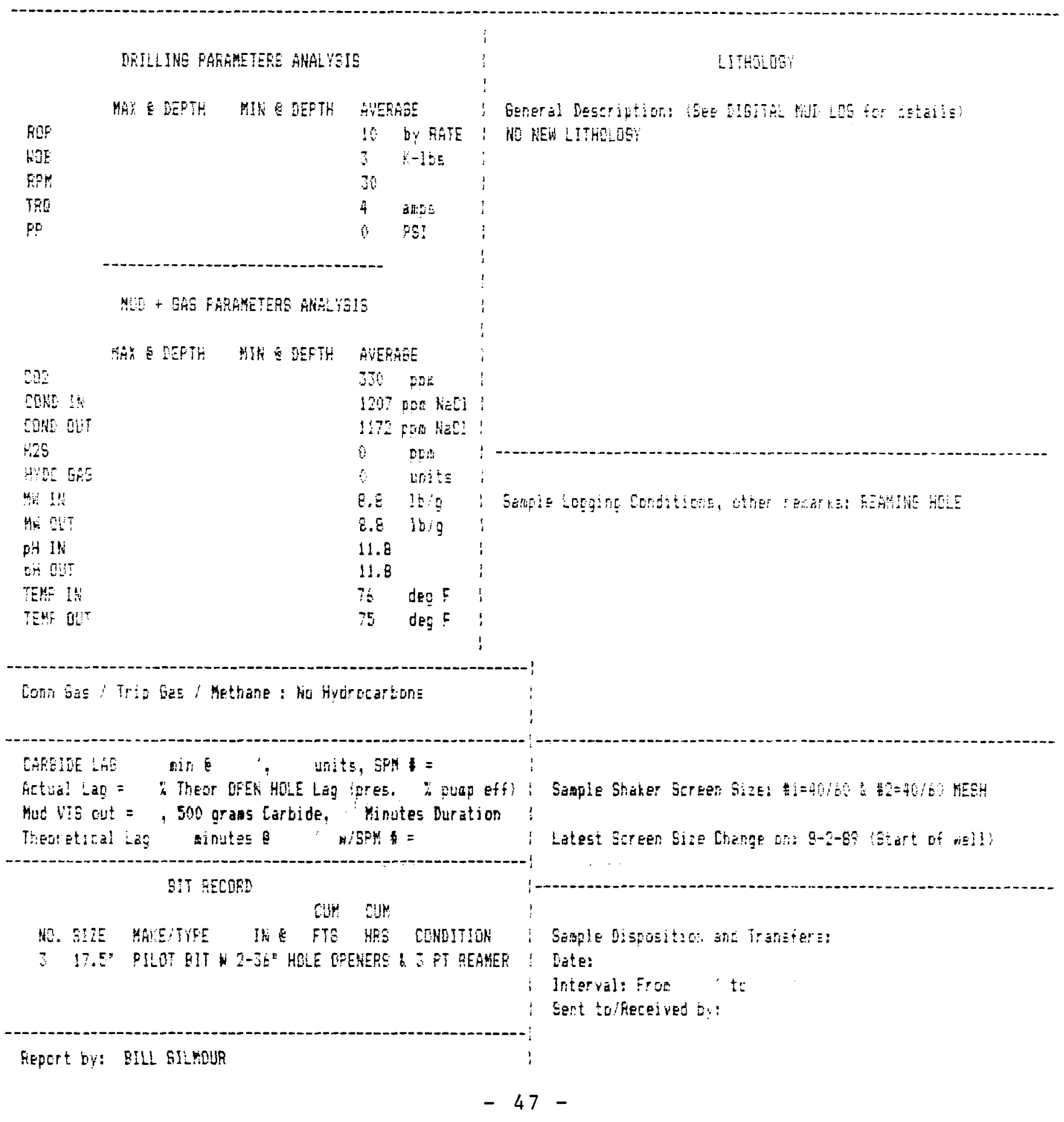




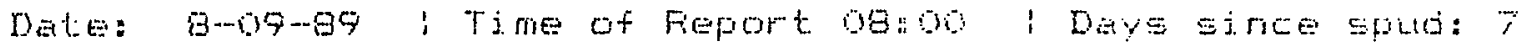

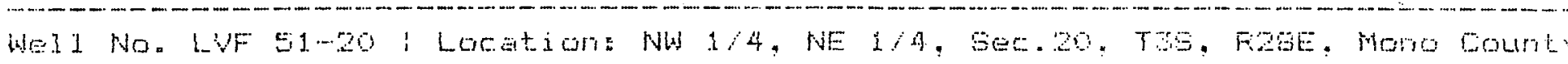

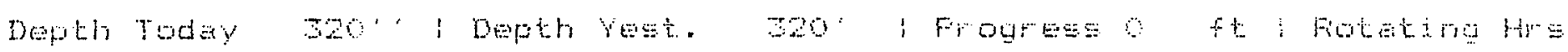

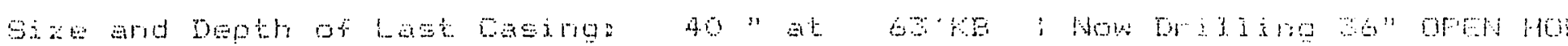

Ei:

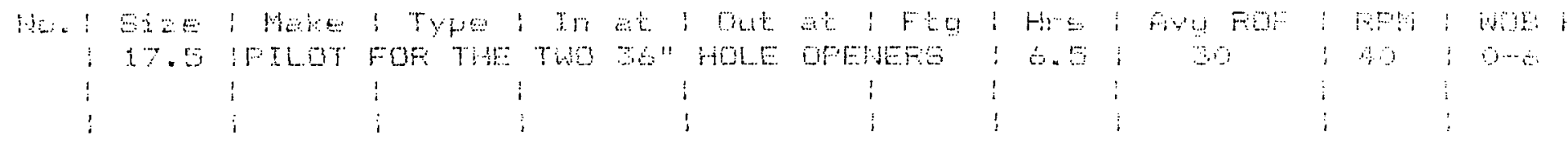

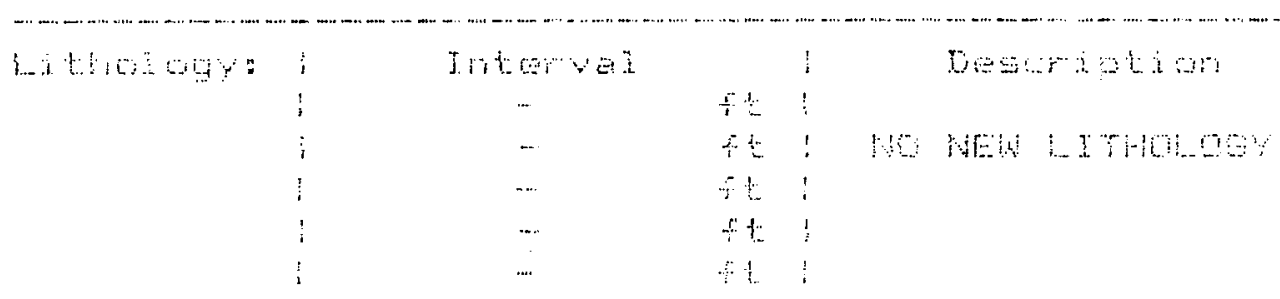

in

Mus

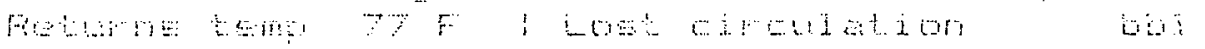

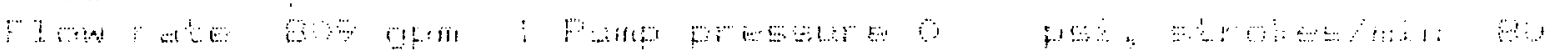

कom

政:

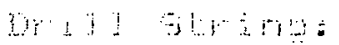

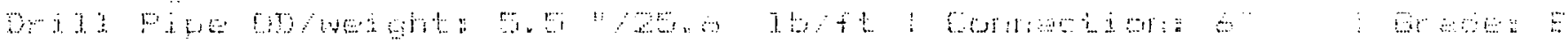

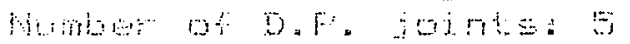

ॠ.

m

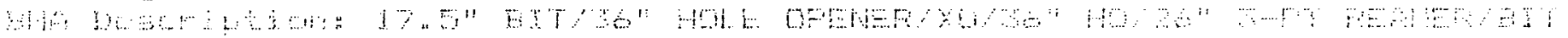

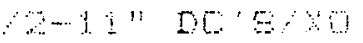

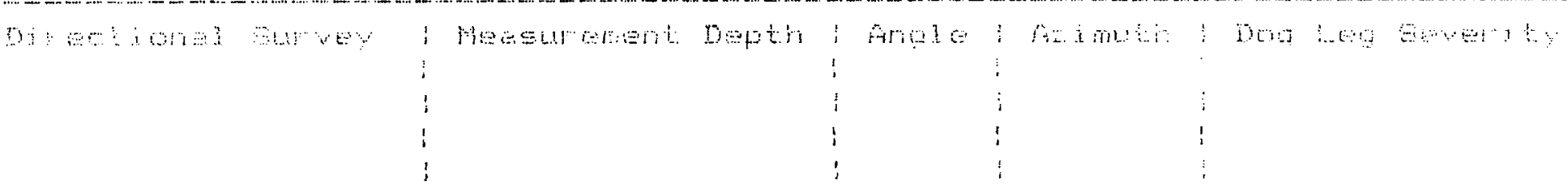

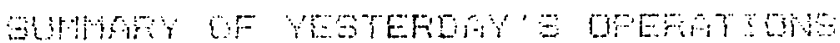

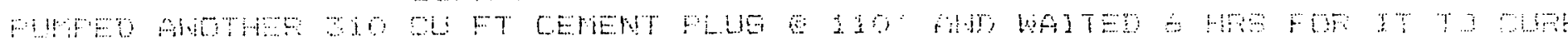

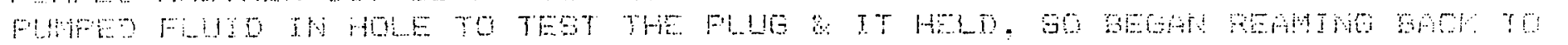

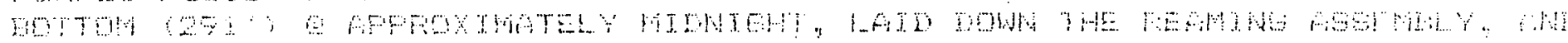

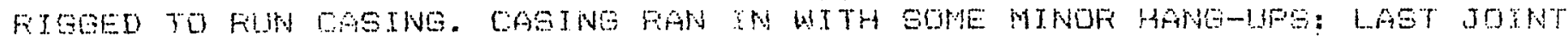

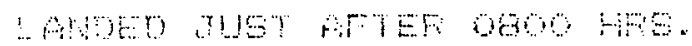

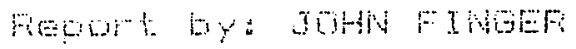




\begin{tabular}{|c|c|c|}
\hline $\begin{array}{l}\text { Report Date } \\
\text { Report Tine } \\
\text { Days Since Spud } \\
\text { Present Depth } \\
\text { Prior Depth } \\
21 \text { Hr Footage } \\
\text { Orilling Hrs } \\
\text { Aug ROP by TIME } \\
\text { ROP is in Feet/H }\end{array}$ & $\begin{array}{l}8-9-89 \\
08: 00 \\
7 \\
320 \\
320 \\
0 \\
0 \\
\text { Hour }\end{array}$ & 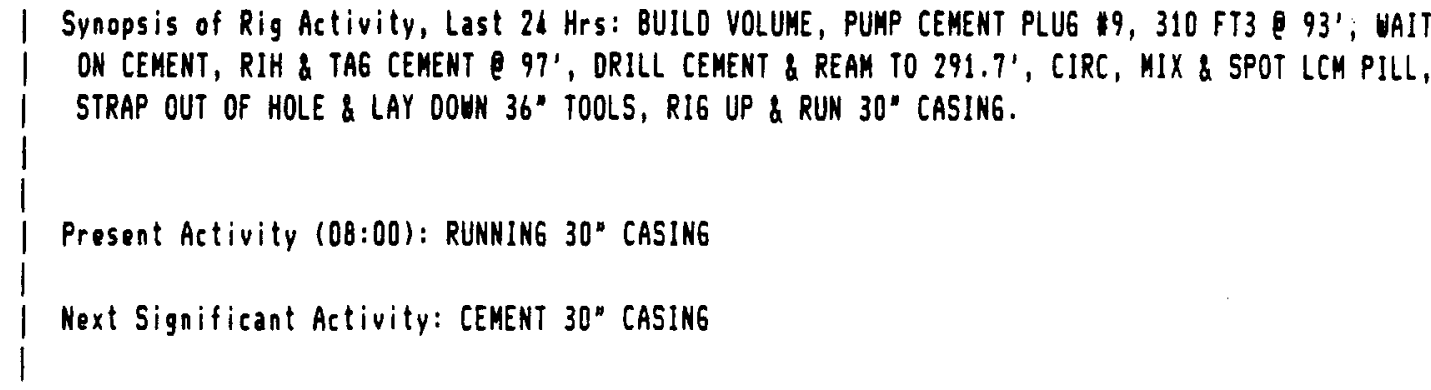 \\
\hline
\end{tabular}

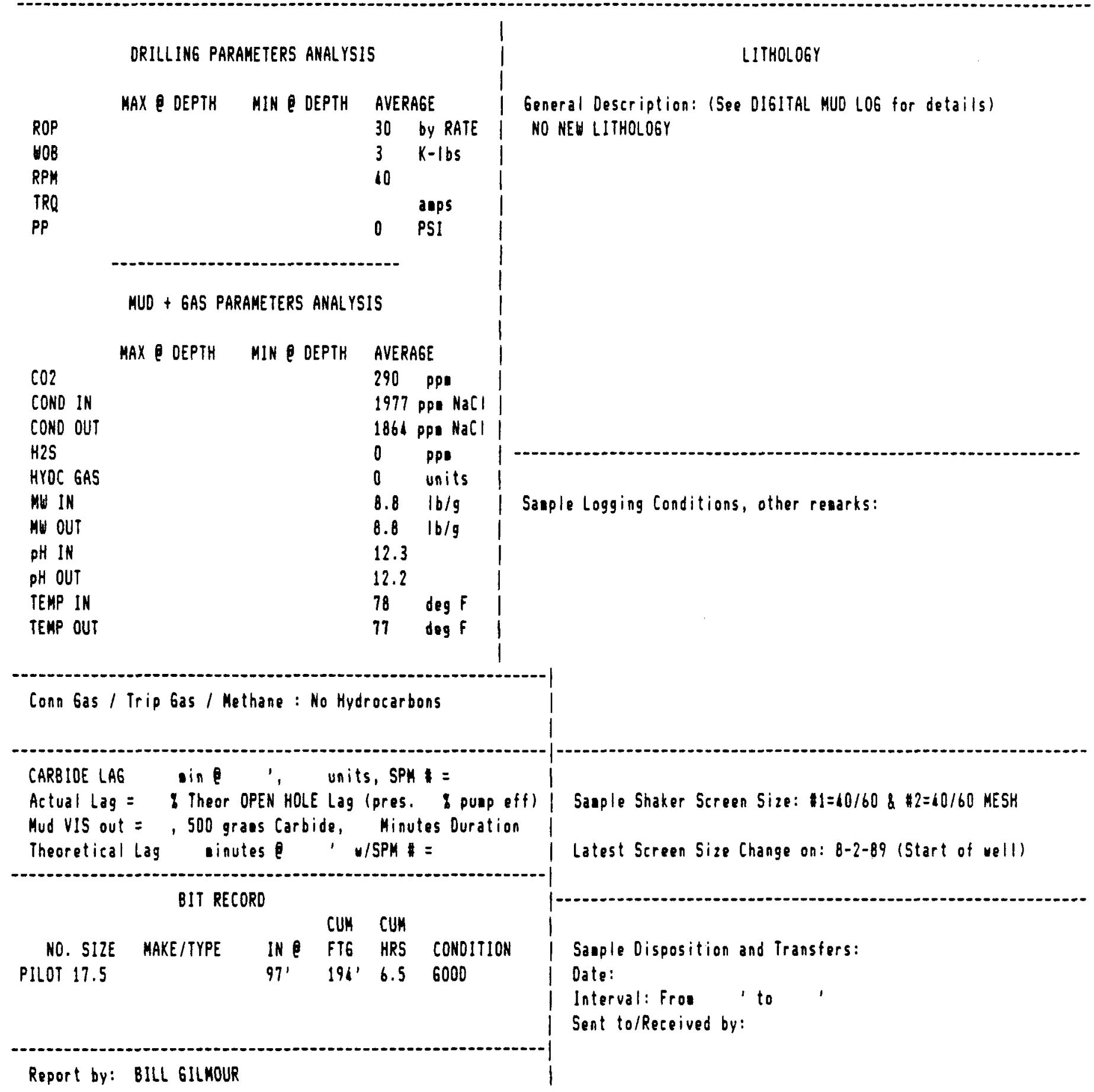


Date: 3-11-89: Time of Feport 08:00 i Diys Eince spud: 7

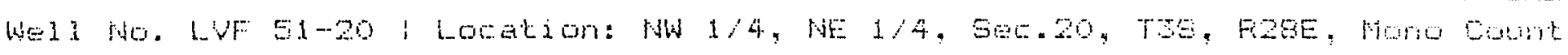

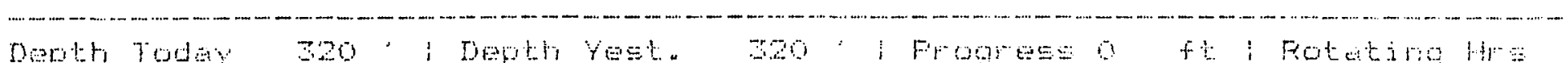

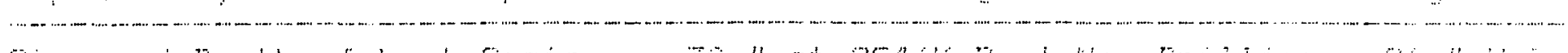

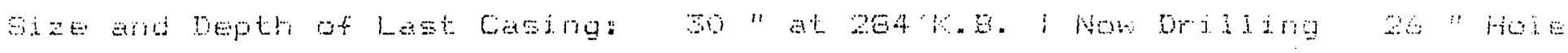
bit Desto

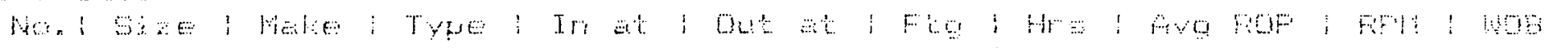

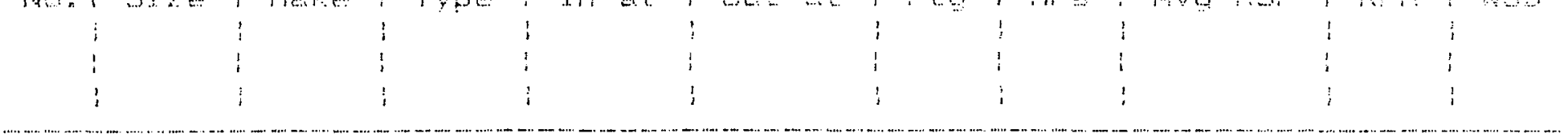

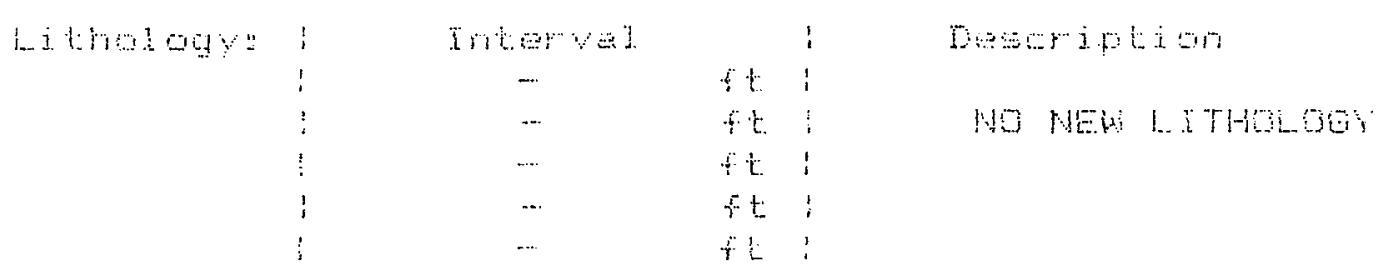

Minising Fludi

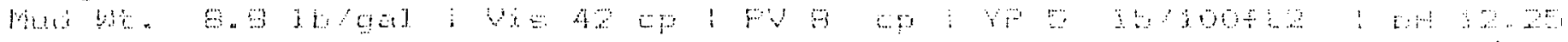

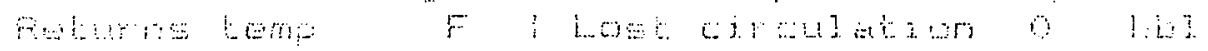

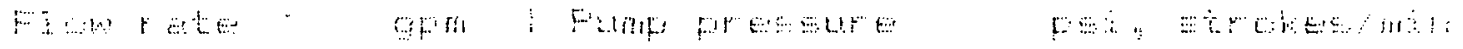

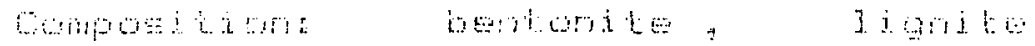

16:

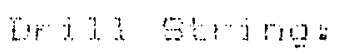

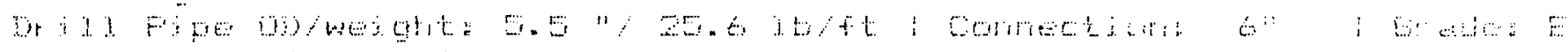

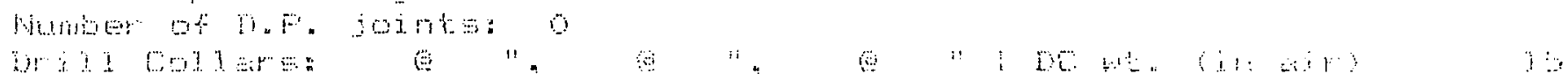

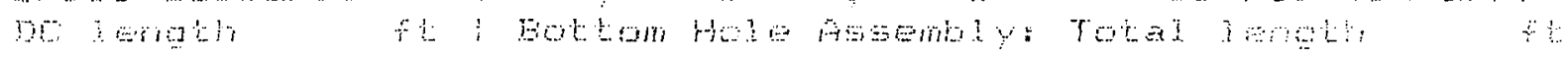

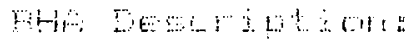

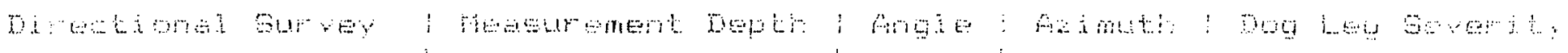

$\begin{array}{ccc}1 & \vdots & \vdots \\ \vdots & \vdots & \vdots \\ \vdots & \vdots & \vdots\end{array}$

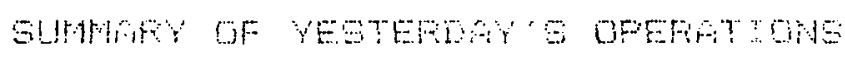

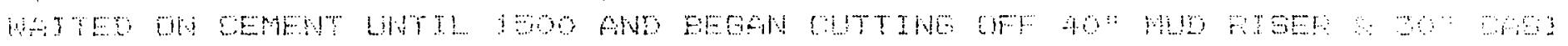

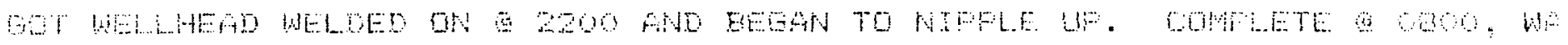
ZWG ON BLH FEFFESENTATIVE TO WTHESS EOF FFESBUFE TEST.

Feport Hy JOHW FIHEEF 
EPOCH HELL LO86ING

DOE/SANDIA MAGMA ENERGY WELL LUF 51-20

DAILY MUD LOG REPORT

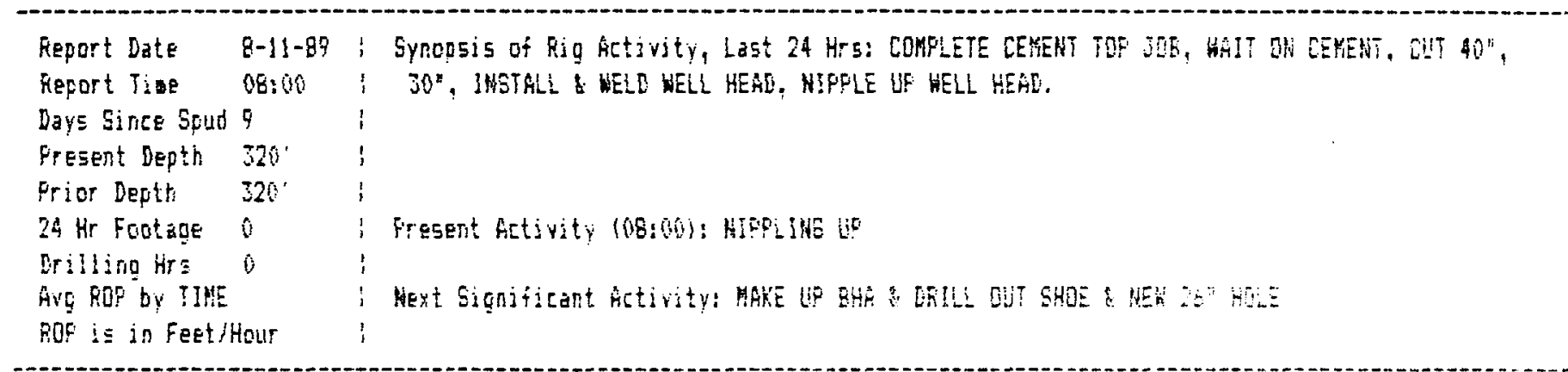

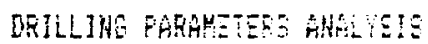

HiH E ISPT

MII S SFFTH

HEFAE

IY RATE

$x-1 b 5$

Wide

Fipt.

T5ti

FF

CQ:

coin is

CONE DUT

H.S

Hin bis

ming it

mivit

titin in

Qin Dis

法? In

TEHF OUT

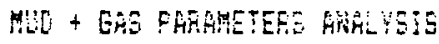

MAR E DEFTH MTH E DEFTH HURABE

anfs

Fs?

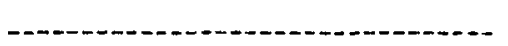

\section{: LThes:}

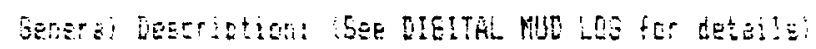

WE NEW LITHOLOS:

!

Cemn bas i Trjo Gas ; Methene : Mo Hydrocartons

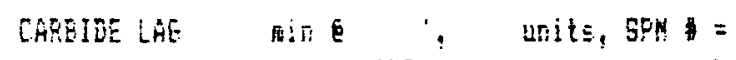

Actua) LaQ $=$ " Theor DPEN HDLE Lab (pres. \& pupp eff) Hai v!s out $=$, sot arage Cartiog, Minutes ouration

Theoretical lag wisutes wiSPM

- Thegretical La

BIT BELORE

W. SIIE MANE:TYPE IHE FTE HRS CONDITIDN
Sampie Shake: Screen Size: $\mathrm{Al}=40100 ; \# 2=40160 \mathrm{MESH}$

Latest Sereen Size Ctionẹe onil Q-2-39 (Start of weil!) (1)

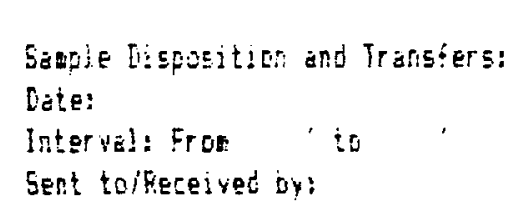




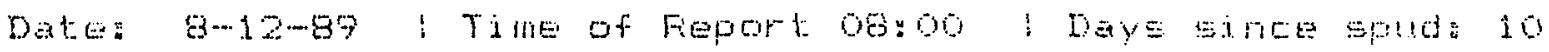

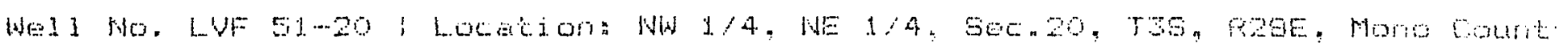

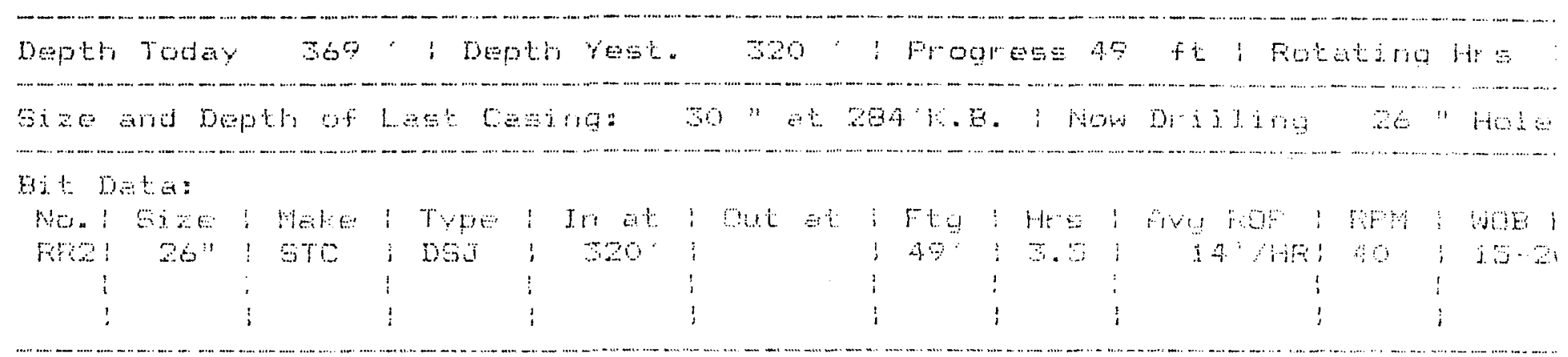

L thenogy

$\operatorname{lnt} s \sin$

अ०

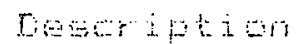

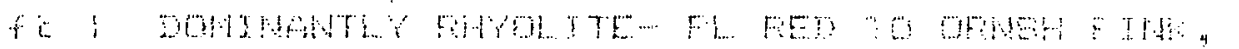

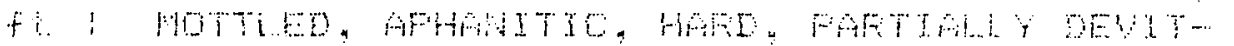

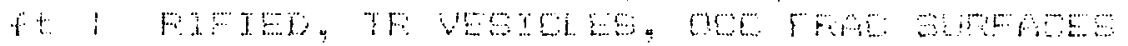

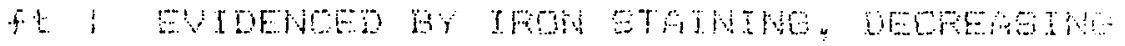

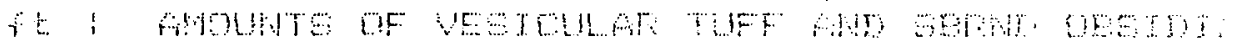

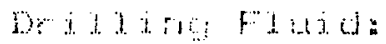

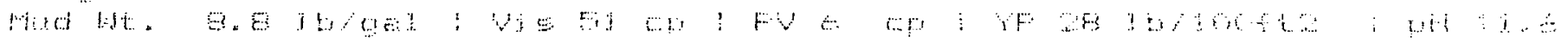

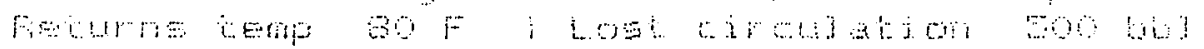

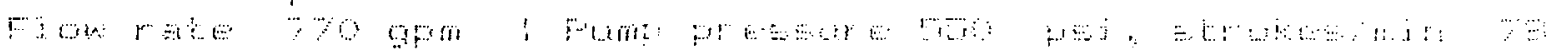

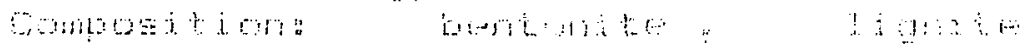

otin:?:

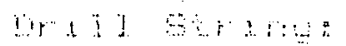

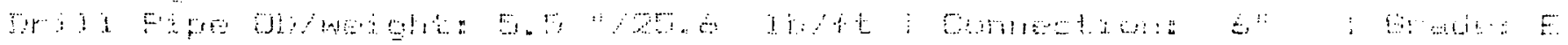

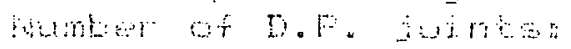

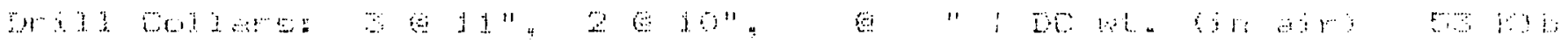

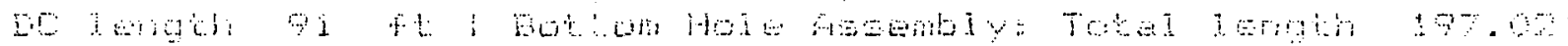

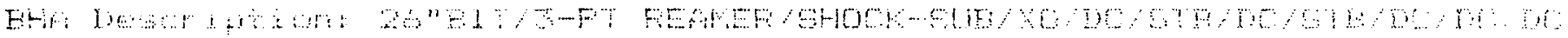

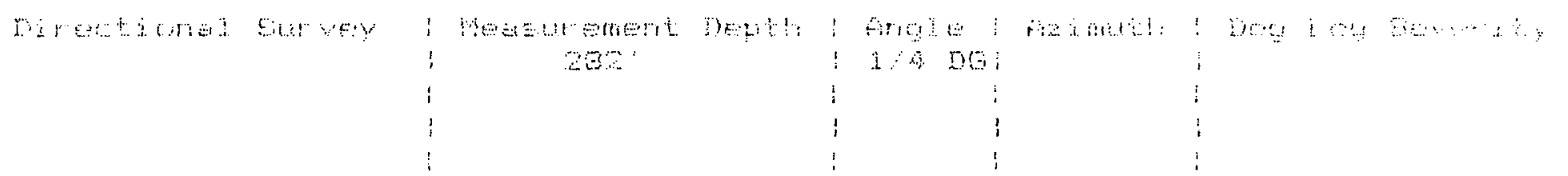

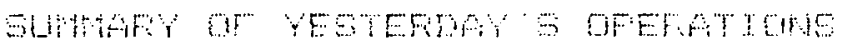

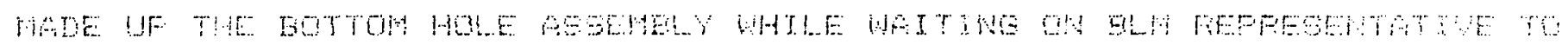

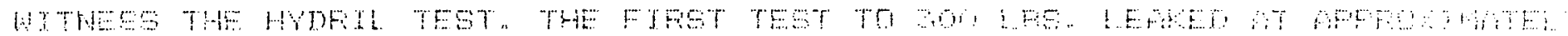

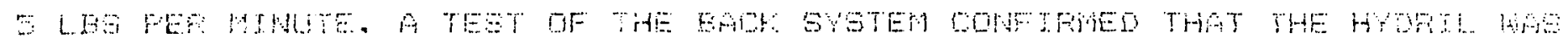

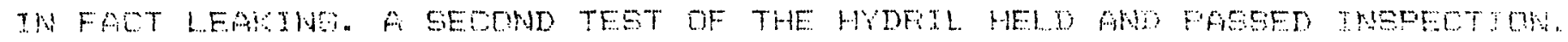

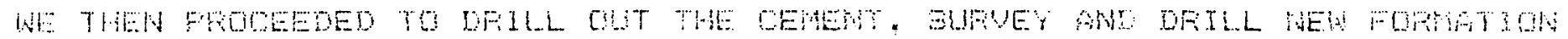

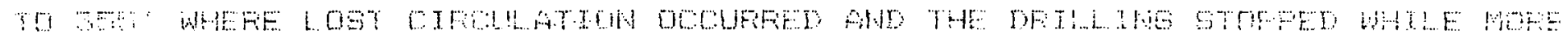

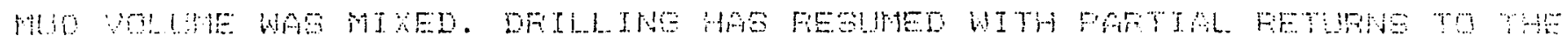

MEEM METH OF $39^{\circ}$

Feport by: For JaOQEGom 
EPOCH MELL LOGGIHG

DOE/SANDIA MAGMA ENERGY WELL LUF 51-20

DAILY MUD LOG REPORT

\begin{tabular}{|c|c|c|c|}
\hline Report Date & $8-12-89$ & i & Synousis of Big Activity, last 24 Hrs: MADE UP BHA, TESTED THE HYBR!L WHICH taILED THE FIRET \\
\hline Feport Tine & $06: 00$ & ! & 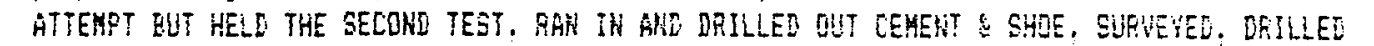 \\
\hline Days Since Spud & 10 & i & 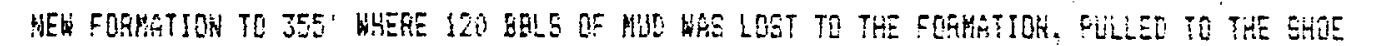 \\
\hline Fresent Depth & 30. & i & 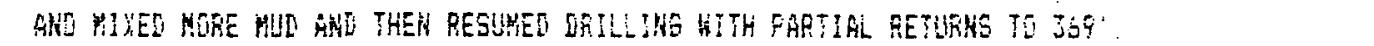 \\
\hline Prior Depth & $320^{\circ}$ & $\vdots$ & \\
\hline $24 \mathrm{hr}$ footuge & $47^{\circ}$ & i & 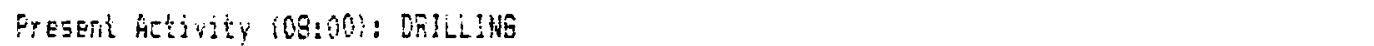 \\
\hline Drílinche & 5.5 & 1 & \\
\hline $\begin{array}{l}\text { AVE ROE bY TIHE } \\
\text { FOF is in FeEt/H }\end{array}$ & $\begin{array}{l}34 \\
30 u r\end{array}$ & $\vdots$ & Neut Significant fetivity: OEILI.......... \\
\hline
\end{tabular}

TRILLIHG PARAMETES AHALYSES

\begin{tabular}{|c|c|c|c|c|c|}
\hline 精: & IEFTH & \multicolumn{2}{|c|}{ MIN 色 SEFFH } & \multicolumn{2}{|c|}{ AUERABE } \\
\hline 50 & $35:$ & 3 & $366^{\circ}$ & $1 E$ & by RETE \\
\hline 24 & 3el' & 12 & $345^{\circ}$ & 1.5 & $x-1$ te \\
\hline 43 & $35 ?^{\prime}$ & 37 & $34^{\circ}$ & 20 & \\
\hline 19 & $366^{\circ}$ & 3 & 321 & $\mathrm{~g}$ & ât \\
\hline 520 & $350^{\circ}$ & 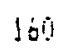 & $309^{\circ}$ & $5 n$ & FE? \\
\hline
\end{tabular}

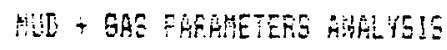

\begin{tabular}{|c|c|c|c|c|c|c|}
\hline \multirow[b]{2}{*}{$\sin$} & \multicolumn{2}{|c|}{ MEH S IERTH } & \multicolumn{2}{|c|}{ MTH E DEFTH } & \multicolumn{2}{|c|}{ AHEFAGE } \\
\hline & $377^{\prime}$ & 347 & 304 & 352 & 359 & op: \\
\hline Cinto If & 1778 & 32 & 87 & $360^{\circ}$ & $143 z$ & por hari \\
\hline cusn suT & 2108 & $322^{\circ}$ & 1012 & $365^{\circ}$ & $167 \%$ & OE HaCl \\
\hline 105 & 8 & & $\theta$ & & $\hat{\imath}$ & ape \\
\hline HUO GAE & 0 & & 0 & & 0 & units \\
\hline$y i n$ : if & 8.8 & $22 !^{\prime}$ & 8.? & $368^{\circ}$ & 5.8 & It: \\
\hline HW GUT & $\bar{c} . \bar{E}$ & & 8.8 & & 8.6 & Ibis \\
\hline pH IN & 12.2 & $321^{\prime}$ & 12.0 & $369^{\circ}$ & 12.1 & \\
\hline DU: & 12.3 & $322^{\circ}$ & 11.5 & $359^{\circ}$ & 12.1 & \\
\hline TES IN & 80 & $3=9^{\circ}$ & 77 & $32 !$ & 79 & $\operatorname{dgg} F$ \\
\hline TEMF ULT & 8 & $357^{\circ}$ & To & $32 !$ & 79 & teg $F$ \\
\hline
\end{tabular}

STHOLOEY

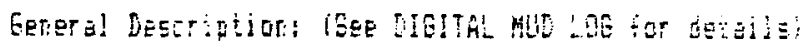

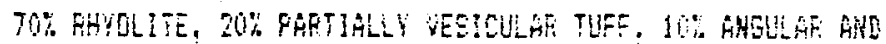

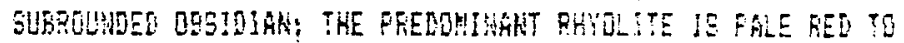

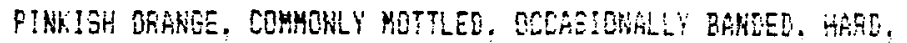

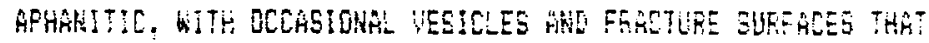

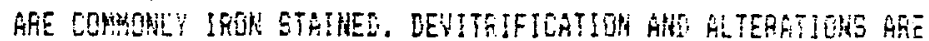

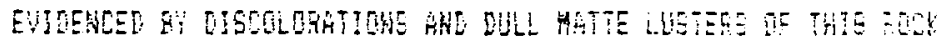
TWE.

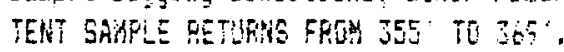

Cumi Eas/Trie Eas Methane : Ho Hudocarbche

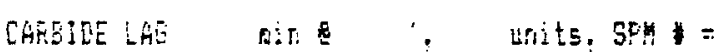

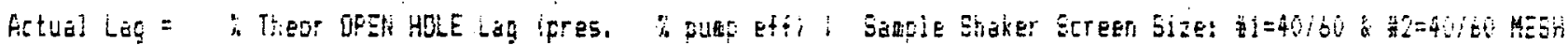
Hud VIS out $=$, s00 orans Carbide, Vinutes Duration

Theoretical Lag atrutes

BIT RECOFO

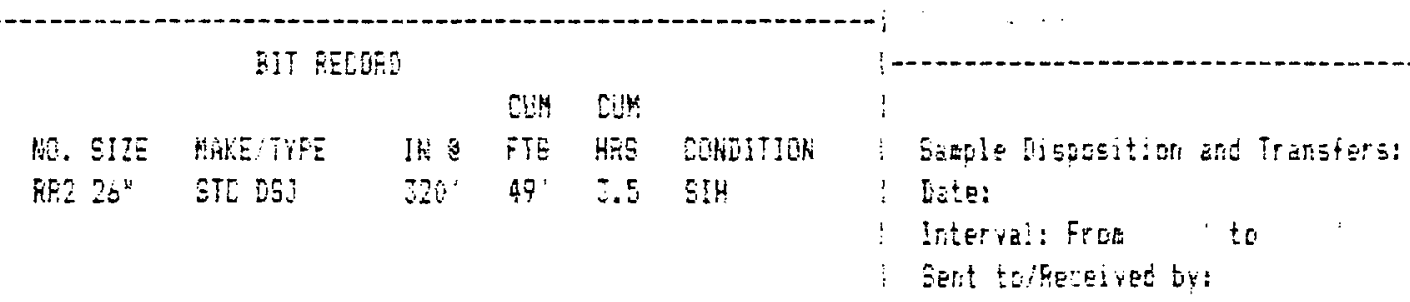

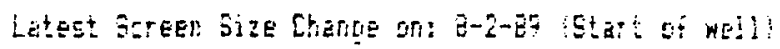


Date: 3-1S-39; Tine of Report 08:00, Days Eince sput: 11

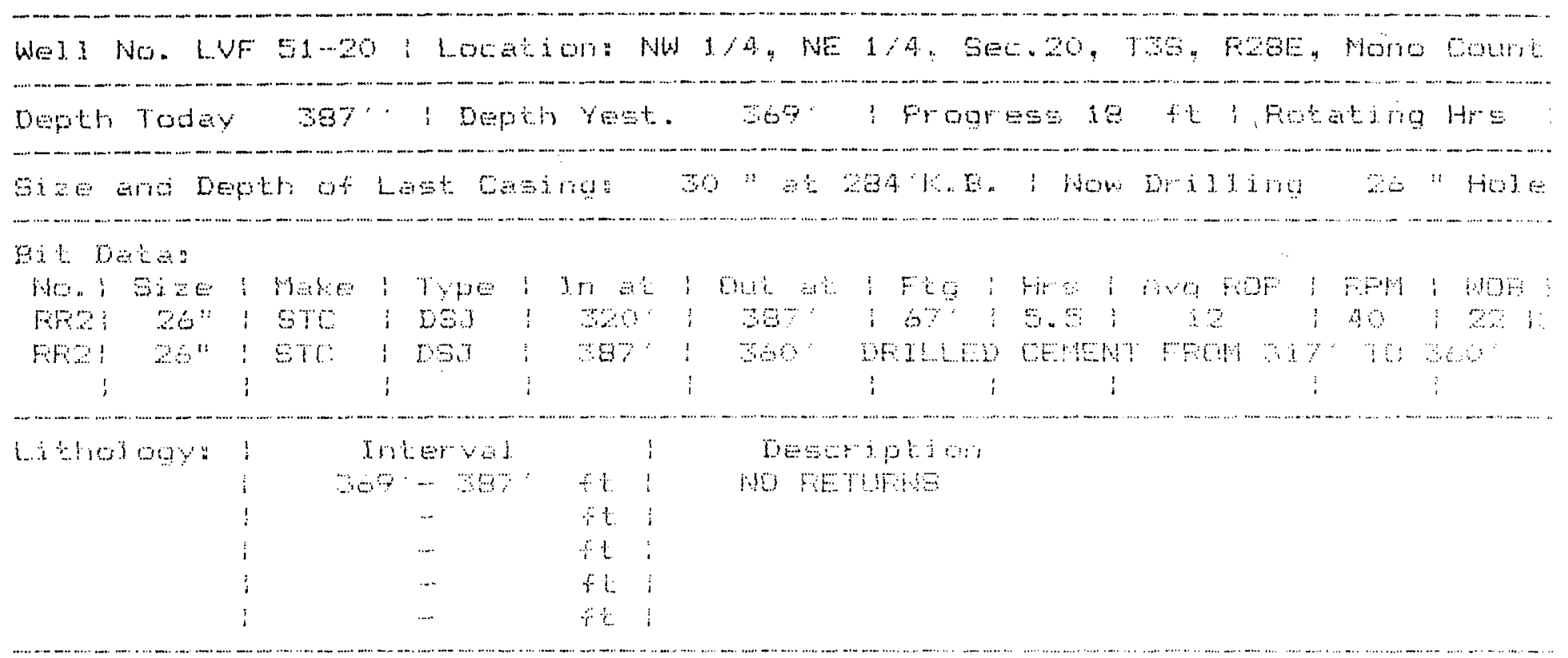

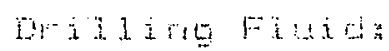

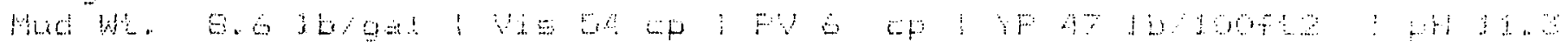

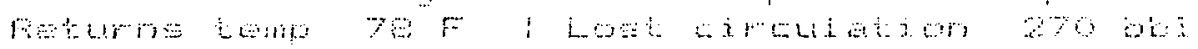

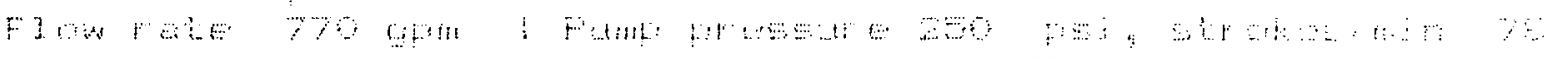

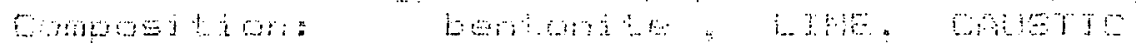

1titer:

Dr.jt

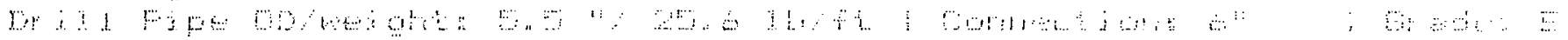

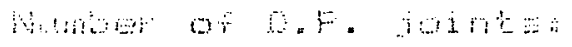

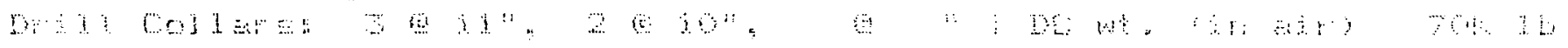

1)

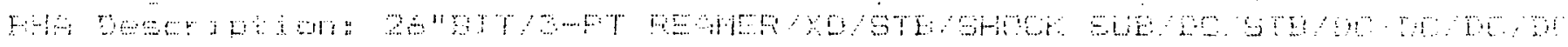

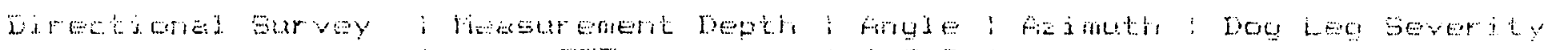

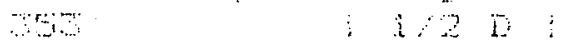

\begin{tabular}{|c|c|c|}
\hline $1 \quad+3$ & $\therefore \quad 1 \quad 2 \quad \square$ & $\vdots$ \\
\hline$\vdots$ & $\vdots$ & $i$ \\
\hline ! & $\vdots$ & $\vdots$ \\
\hline$\vdots$ & 1 & $\vdots$ \\
\hline
\end{tabular}

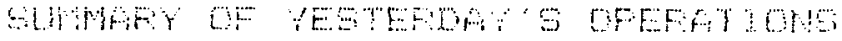

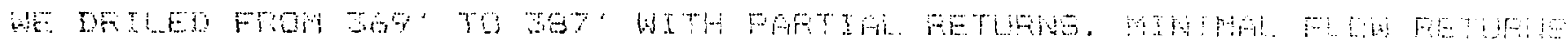
UE

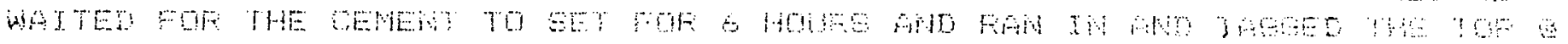

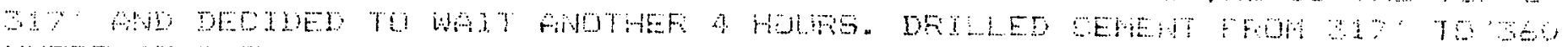

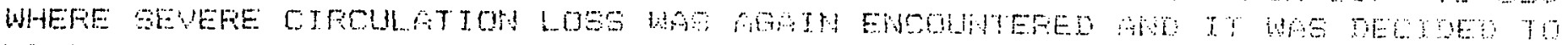

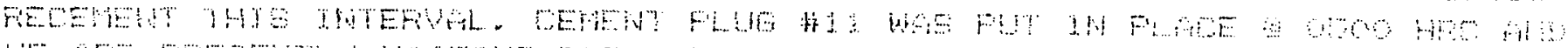

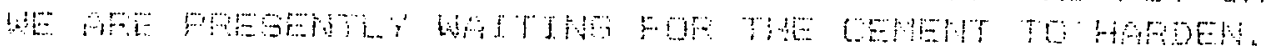

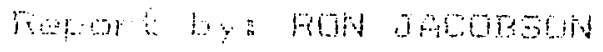


EPOCH HELL LOEGING

DOE/SANDIA MAGMA ENERGY WELL LVF 51-20

DAILY MUD LOG REPORT

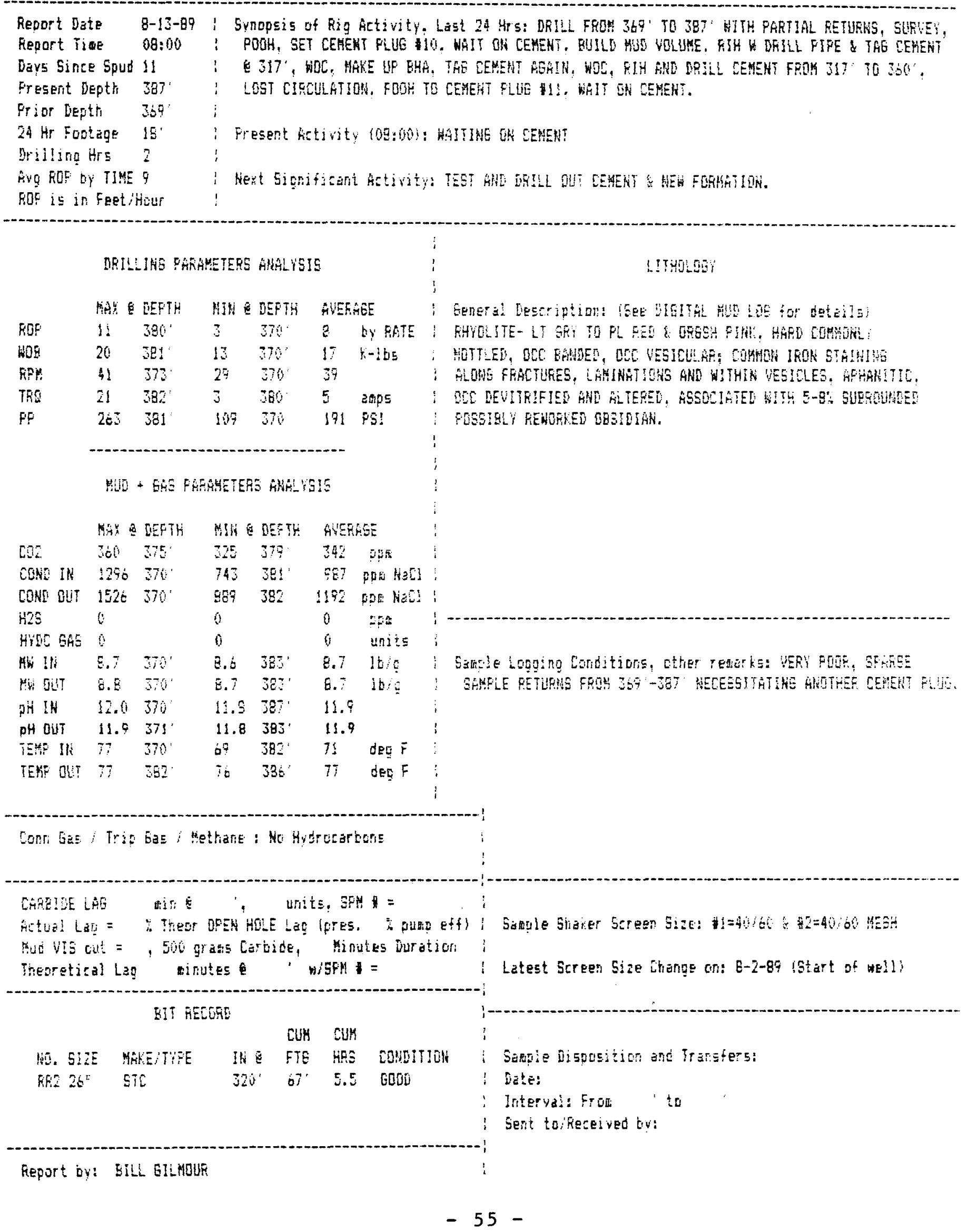


Date: 8-14-89 i Time of Feport oe: oo i Deys since spura is

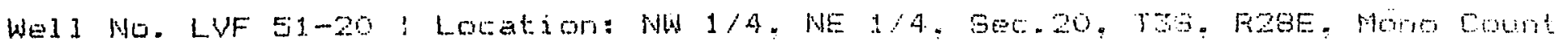

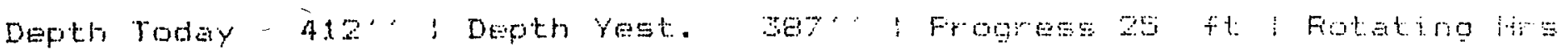

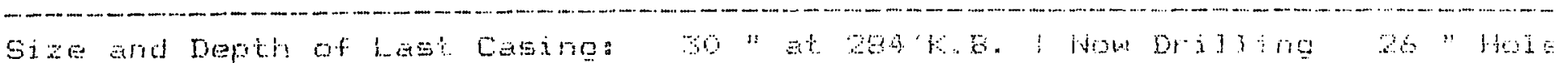

Sit. Dit:

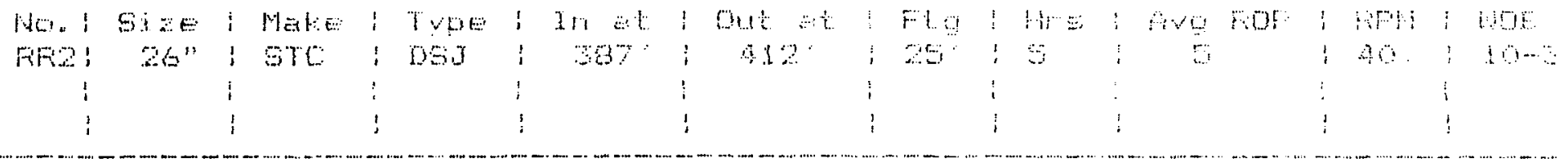

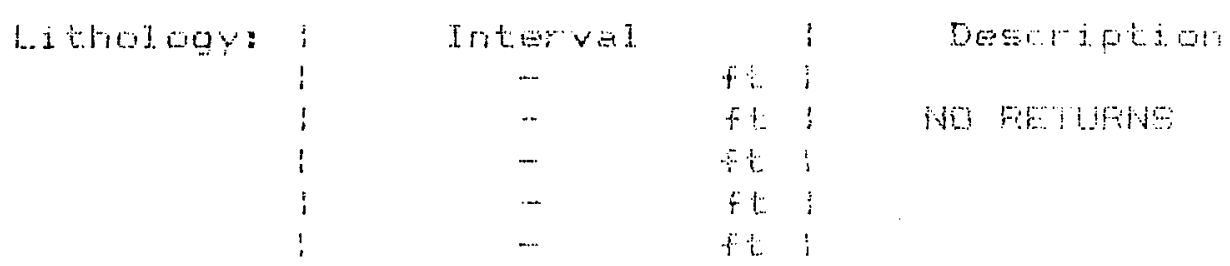

Din 11 ing Flude:

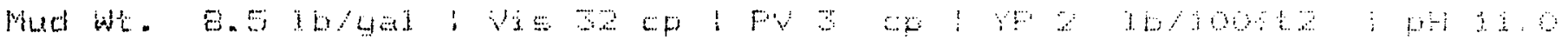

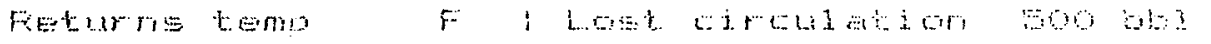

Fow rate baj om

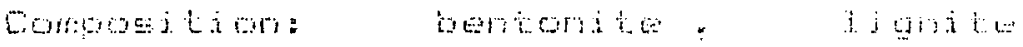
Otilisin:

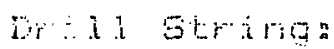

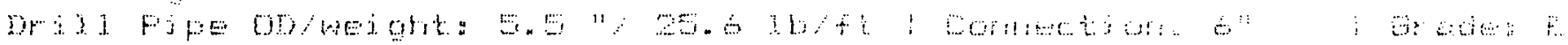
Nubter of D.F. imint:

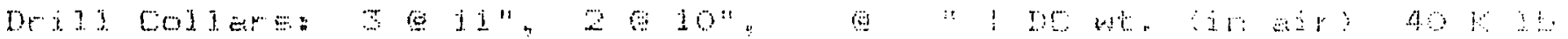

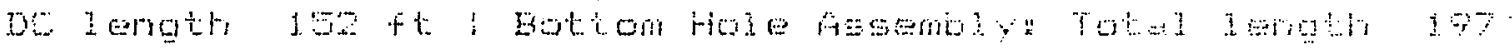

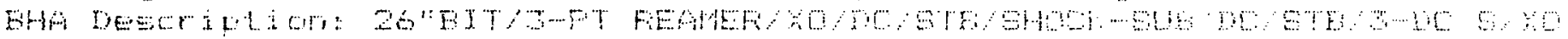

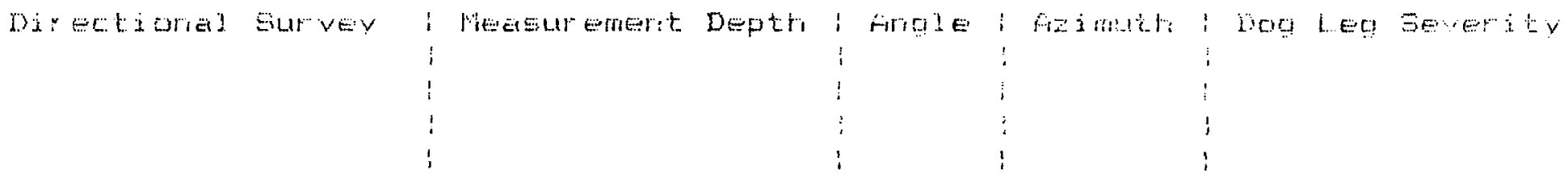

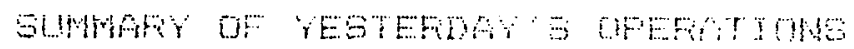

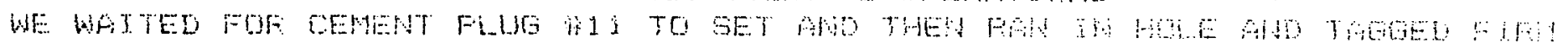

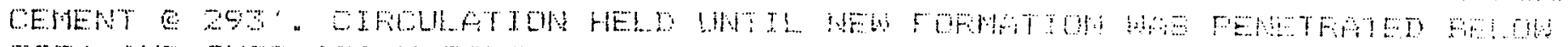

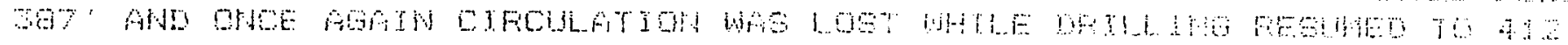

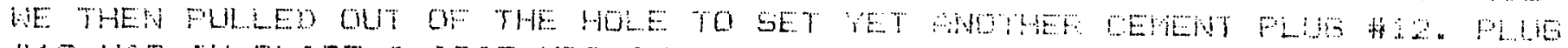

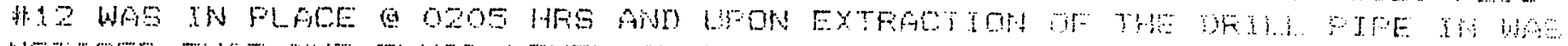

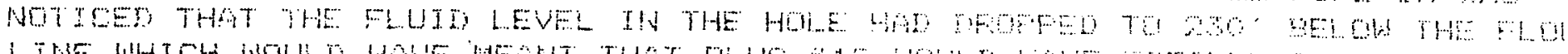

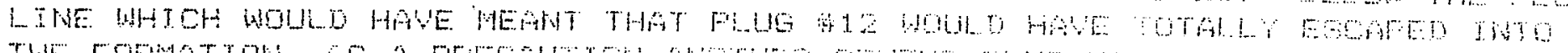

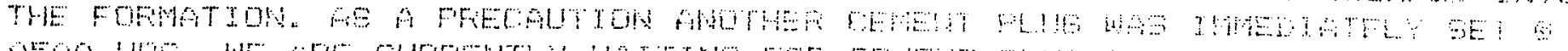

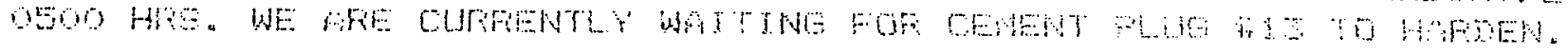

Feport by: ROH JACOESOH 
EFOOH WEL LOSEINE

DOE/SANDIA MAGMA ENERGY WELL LVF \$1-20 DAILY MUD LOG REFDFT

\begin{tabular}{|c|c|c|c|}
\hline Feport Date & $8-14-89$ & $\vdots$ & 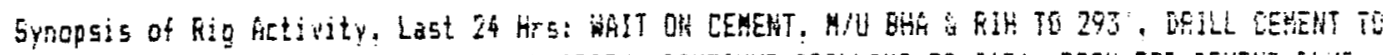 \\
\hline Revort Tiae & $08: 00$ & 1 & 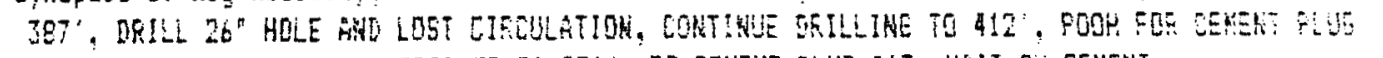 \\
\hline Doys Sitres Soud & $! 2$ & $i$ & 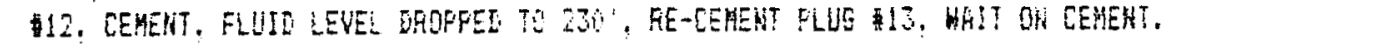 \\
\hline Ergent Detith & 412 & i & \\
\hline Frior Depth & $3 T^{\prime}$ & $\vdots$ & \\
\hline $24 \mathrm{Hr}$ Footaţe & 25 & $!$ & 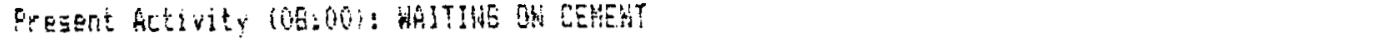 \\
\hline Drilling Hre & 5 & $i$ & \\
\hline $\begin{array}{l}\text { FUQ ROE by TIKE } \\
\text { ROF is i Fegt: }\end{array}$ & $\begin{array}{l}5 \\
H o u r\end{array}$ & $\vdots$ & 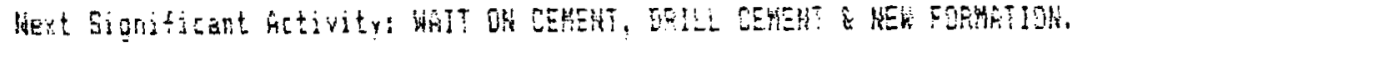 \\
\hline
\end{tabular}

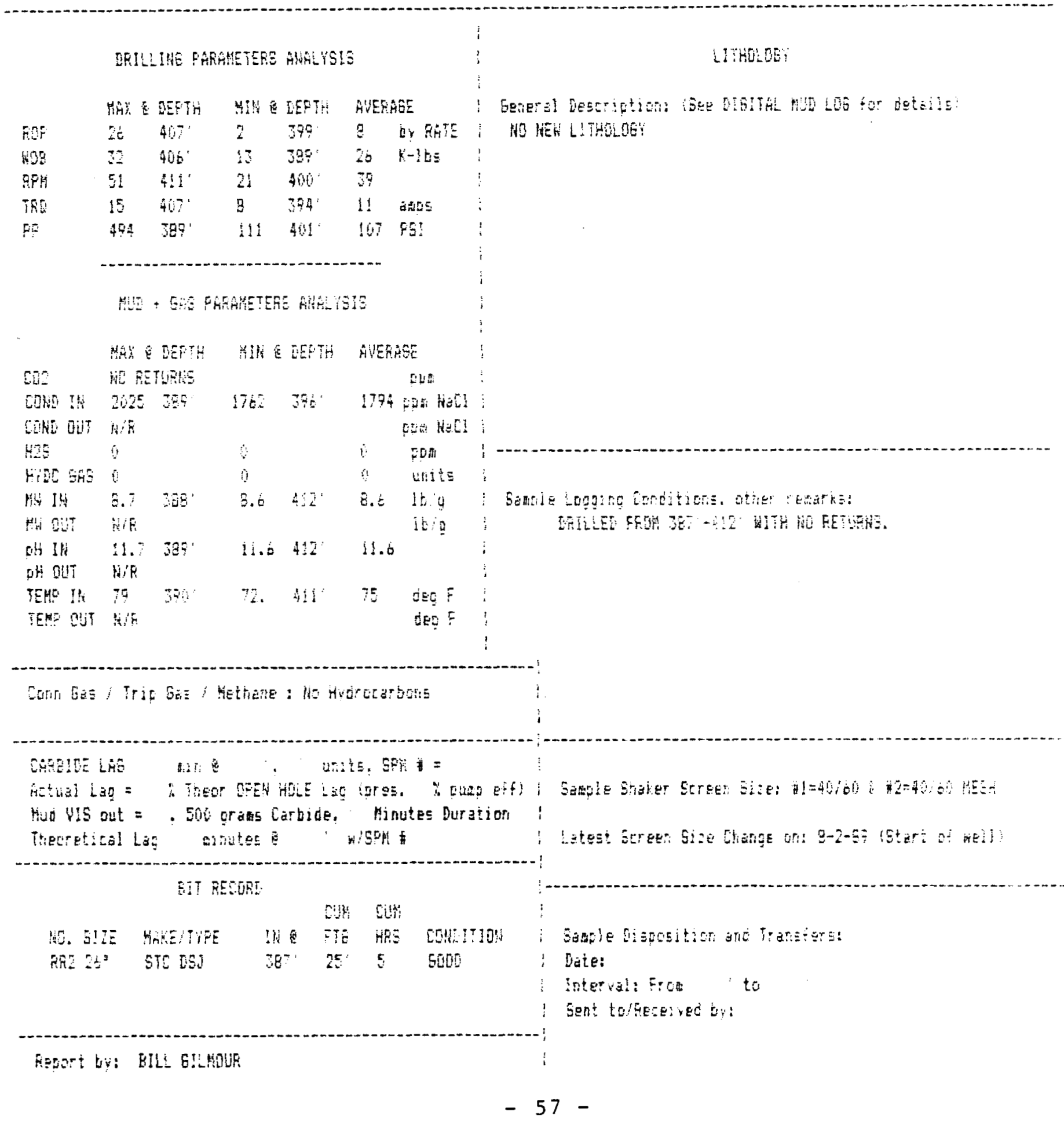




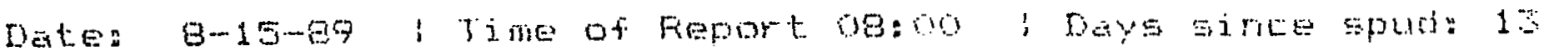

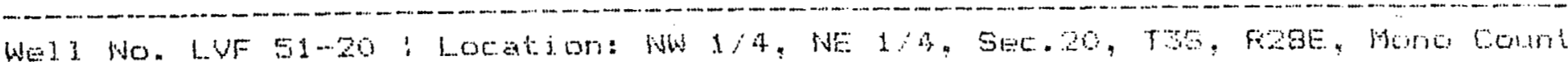

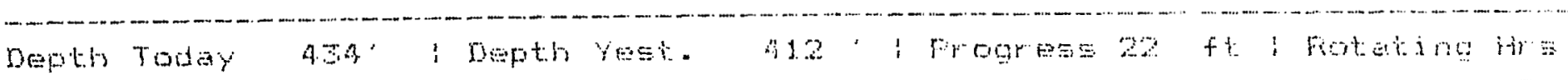

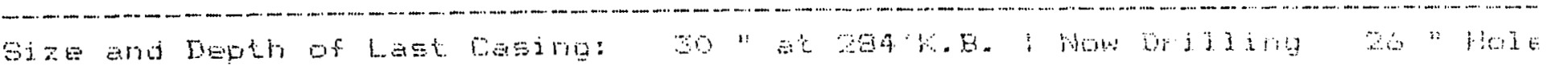

mil Litis:

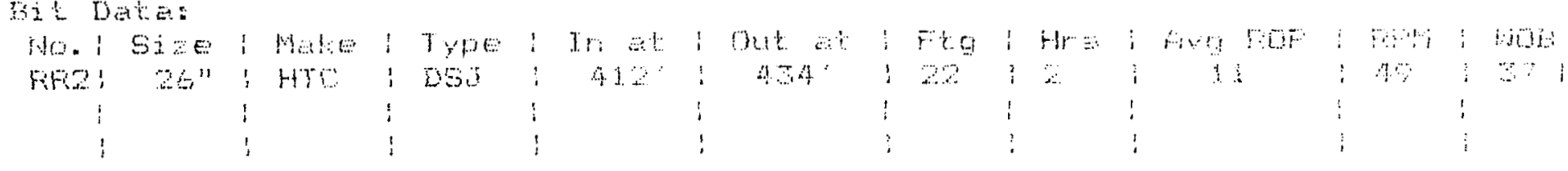

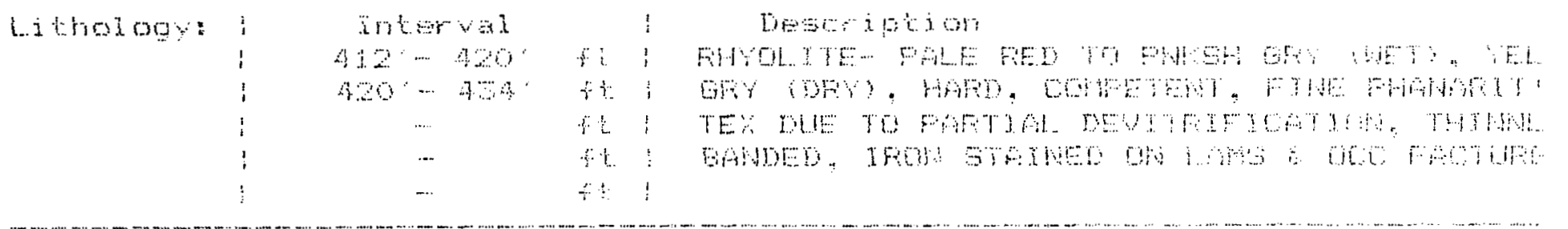

Drizing Fiuid:

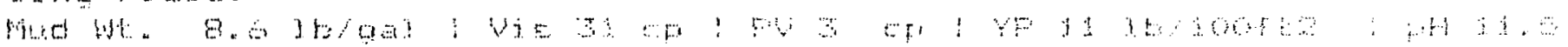

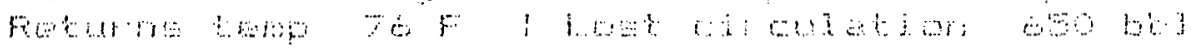

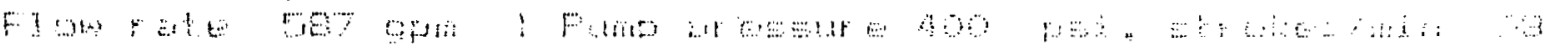

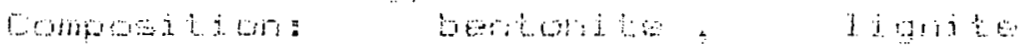

otrie: :

Drail String:

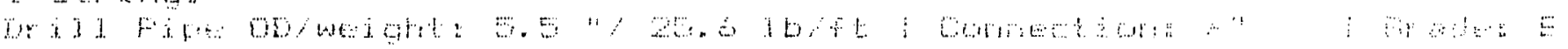

Number ot D.F. juints:

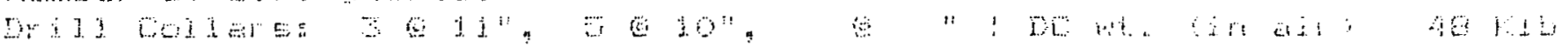

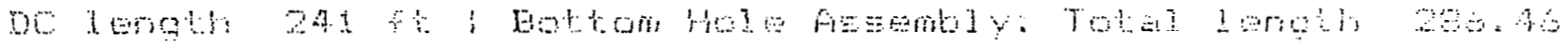

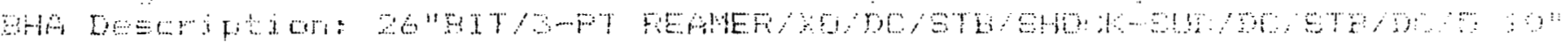

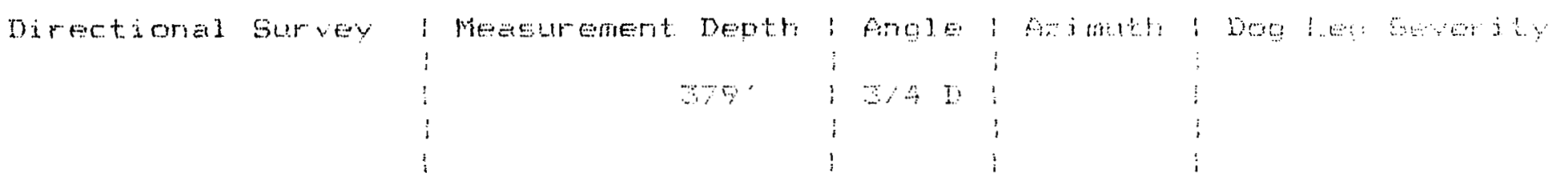

SWMHAY OF YESTERDAY 9 OFEFHTIOH

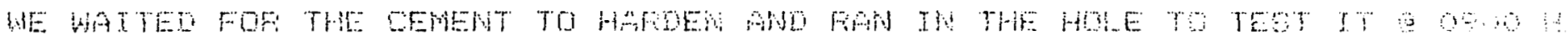

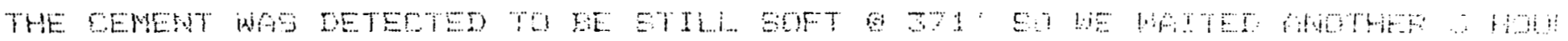

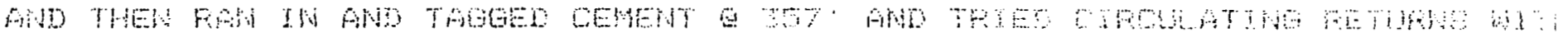

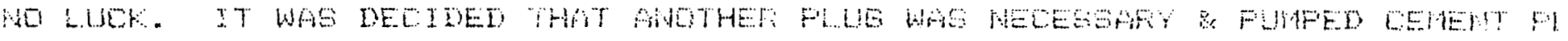

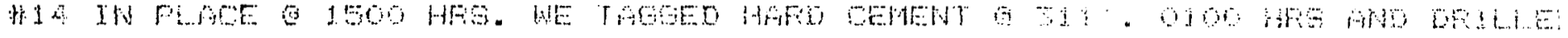

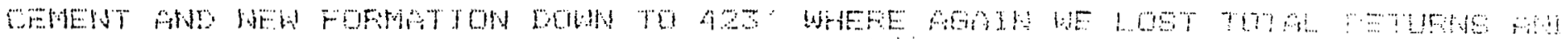

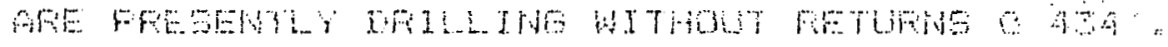

feport by: Ron jacoseon 
EPOCH WELL LOGGINE

DDE/SANDIA MAGMA ENEFGY WELL LVF 51-20 DAILY MUD LDG FEPDRT

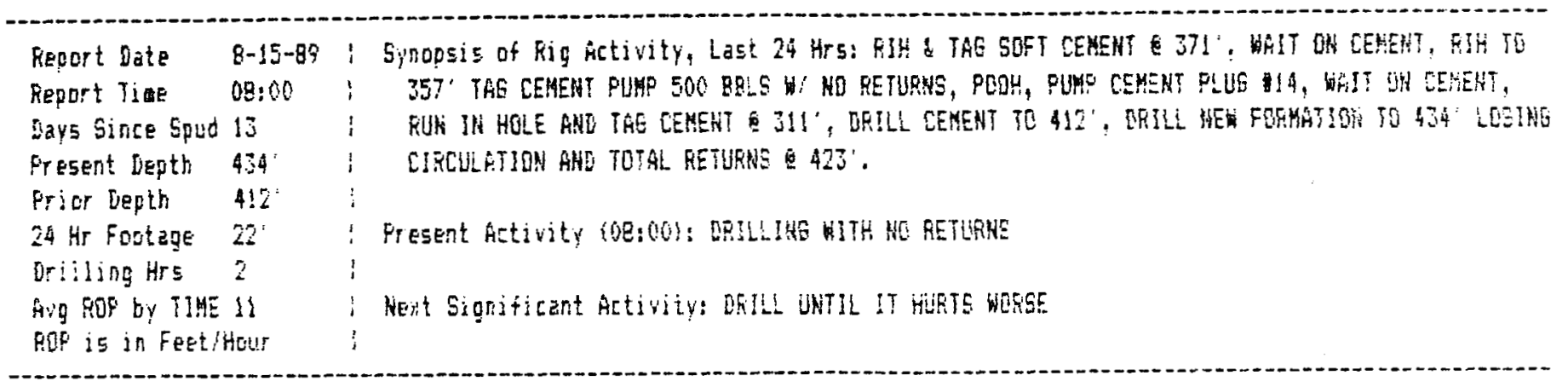

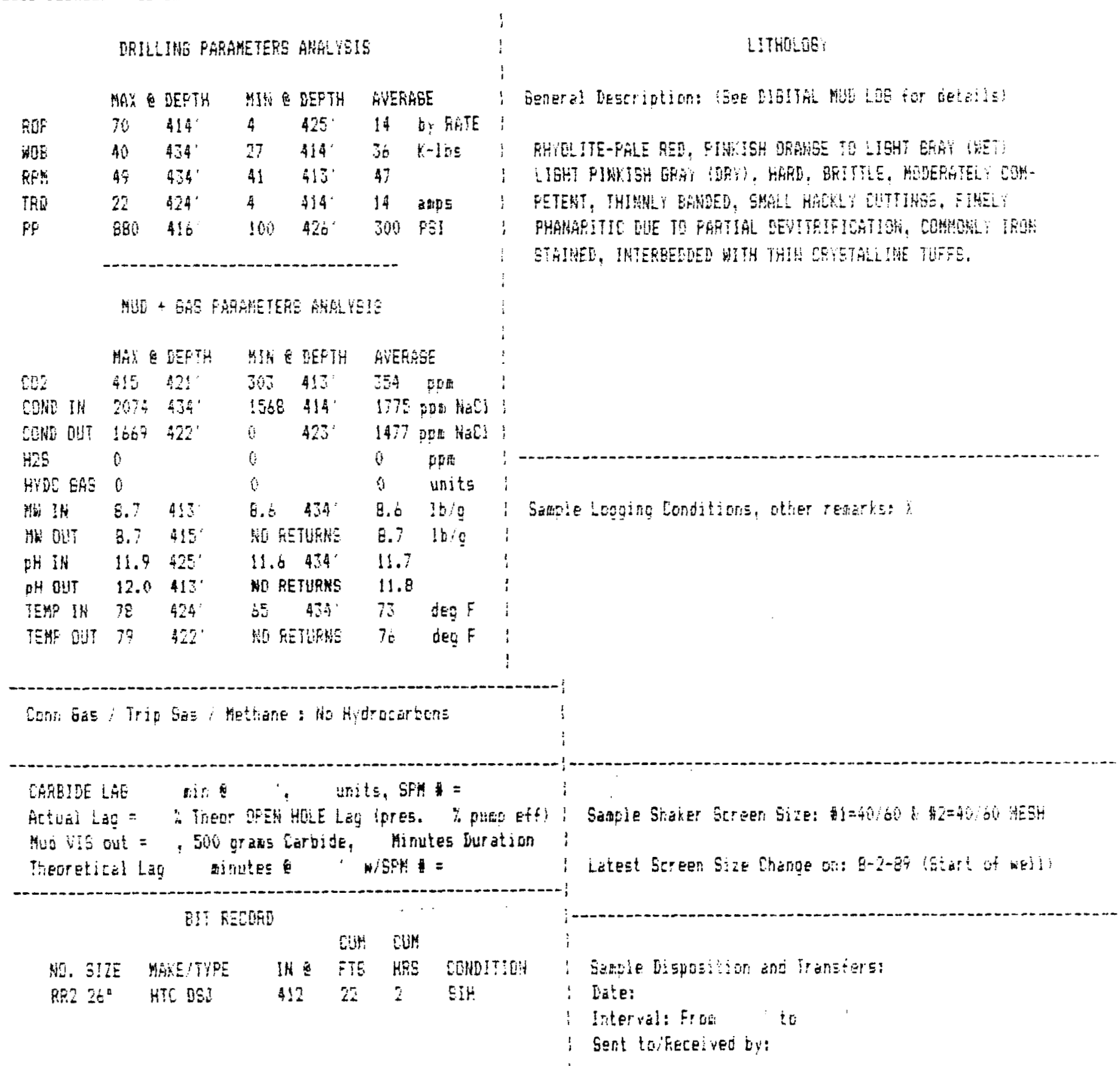




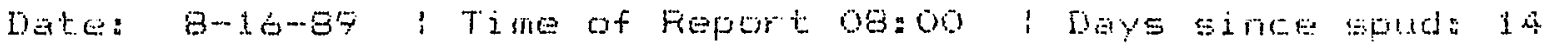

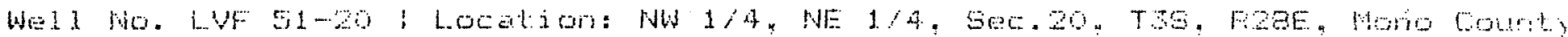

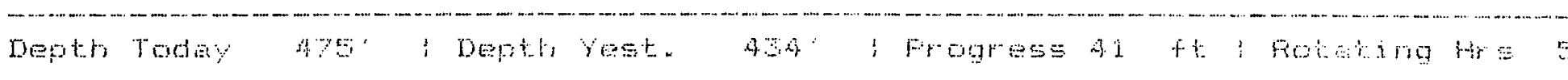

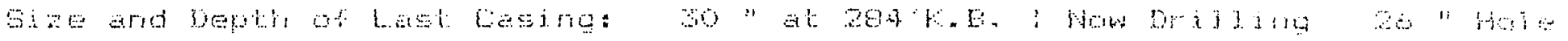

Etit Daten

Ho :

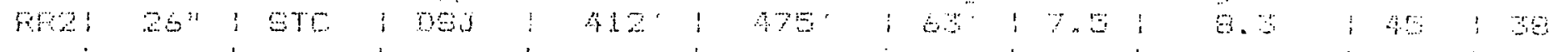

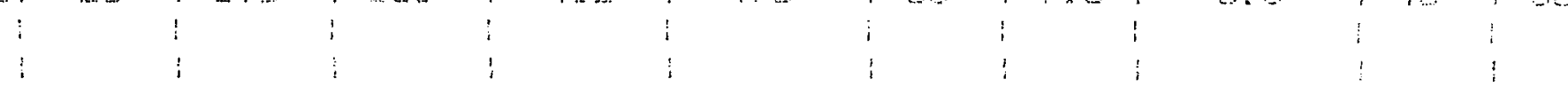

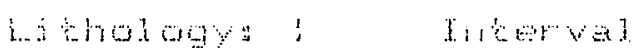

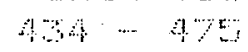

$f t$
$f t$
$+b$
$f t$

Nin $\mathrm{EH} 1 \mathrm{H}$

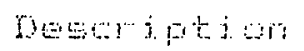

[3)

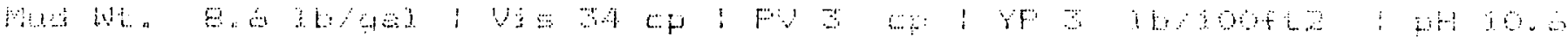

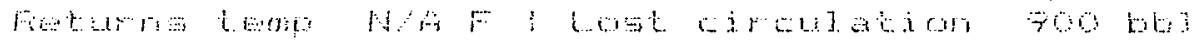

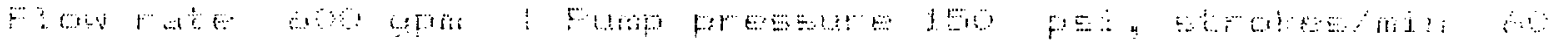

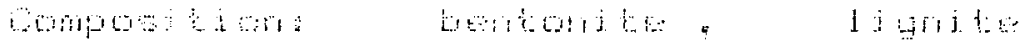

atim?

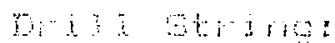

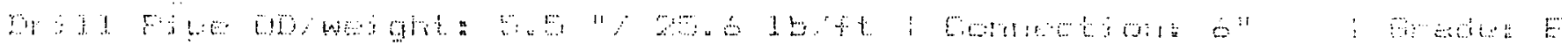

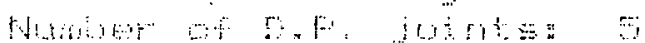

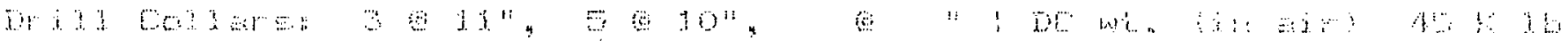

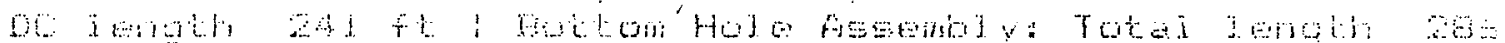

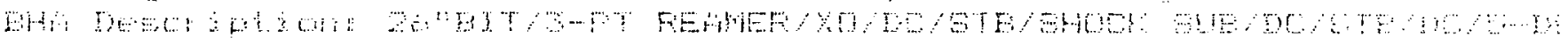

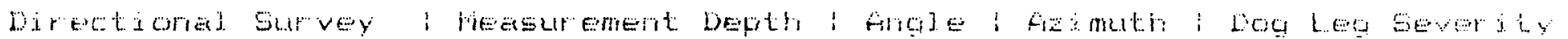

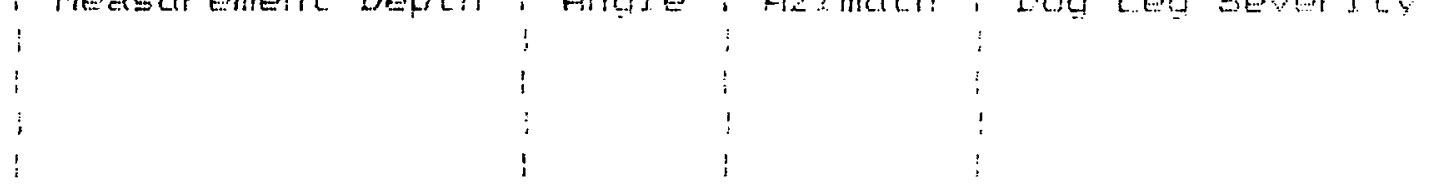

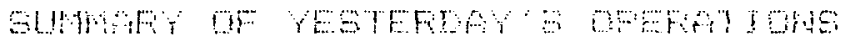

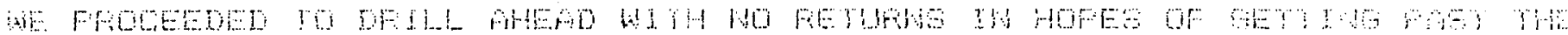

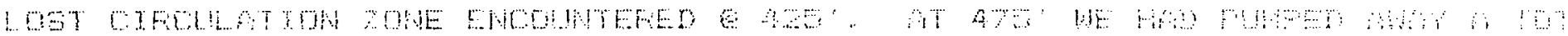

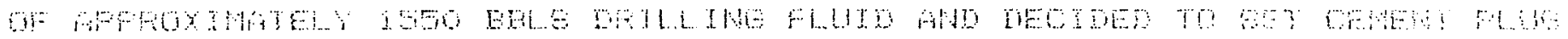

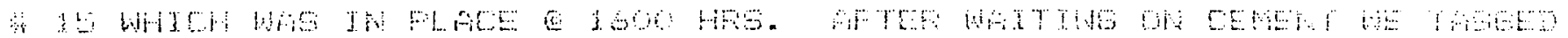

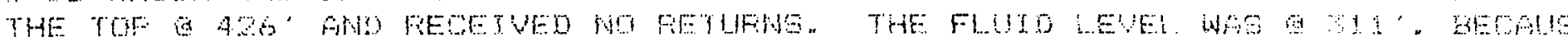

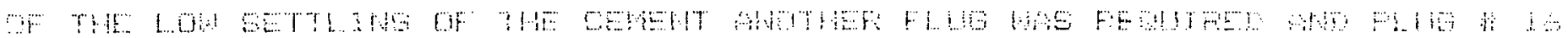
Wha of

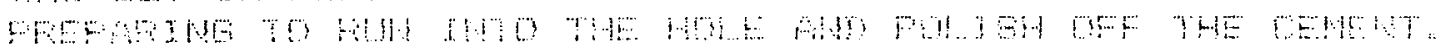

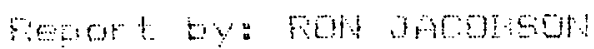


EFDCH WELL LOGGJNG

\section{DOE/SANDIA MAGMA ENEFGY WELL LUF 51-20 DAILY MUD LOG REFORT}

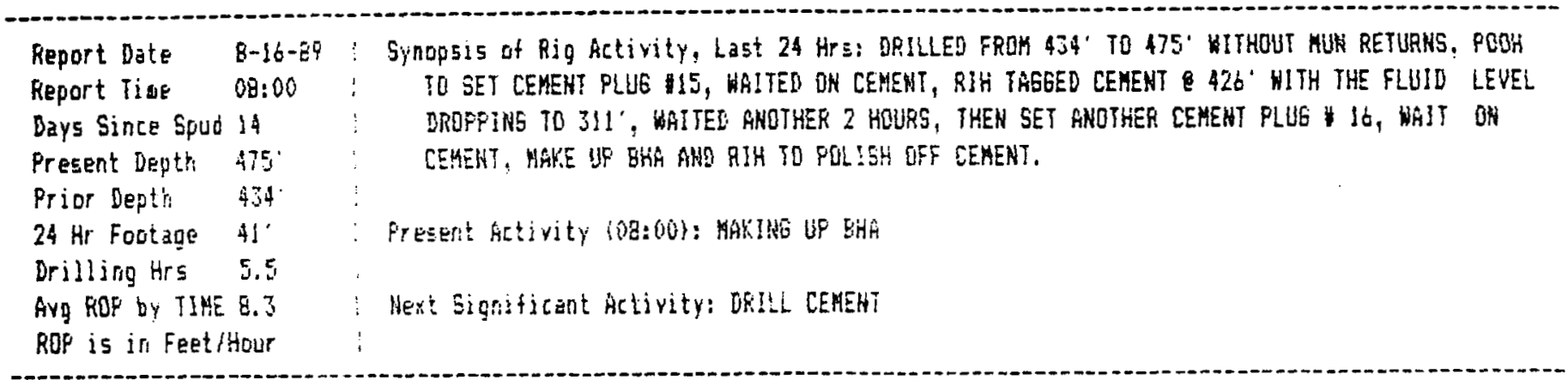

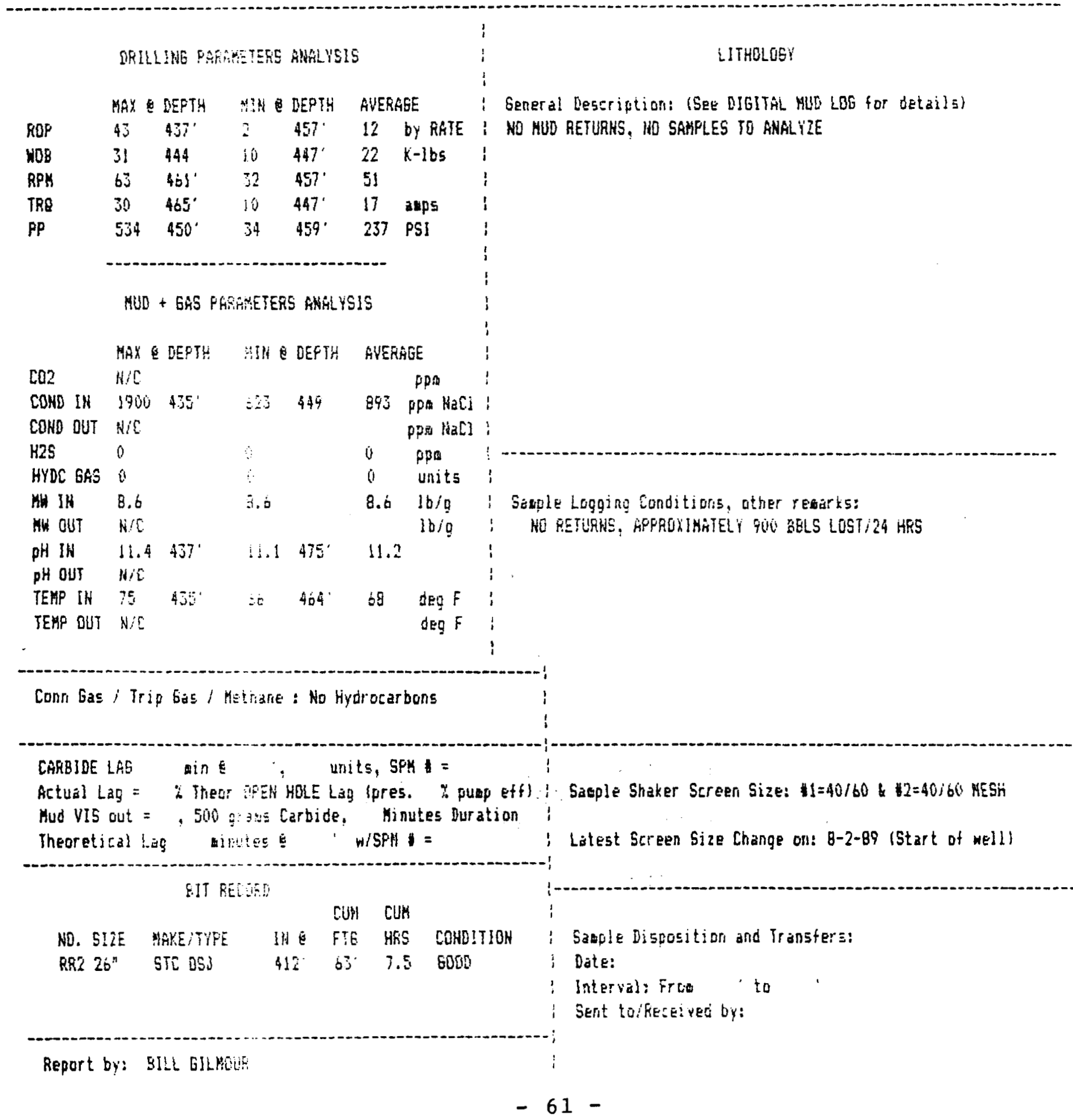




\section{Date: 6-17-39 i Time of Feport obso i Deys sibce spudn is}

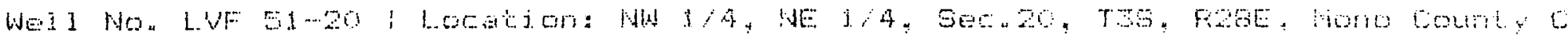

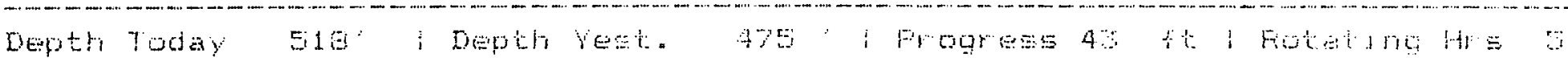

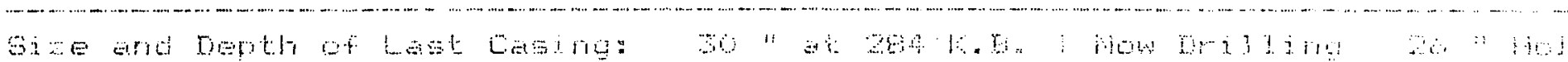

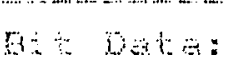

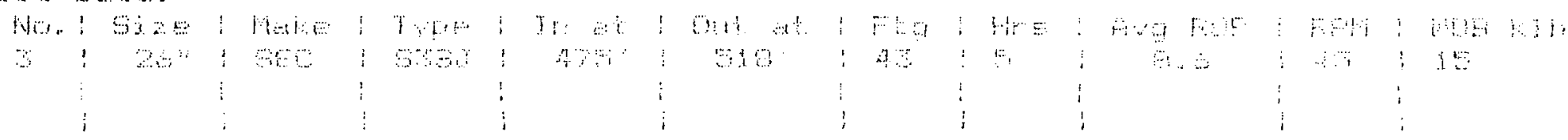

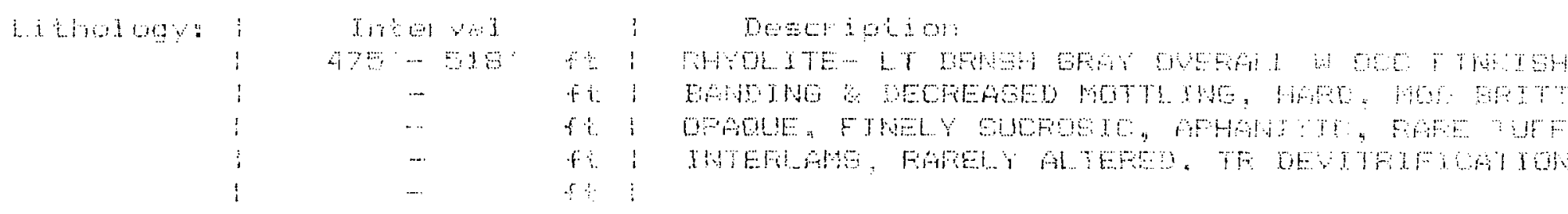

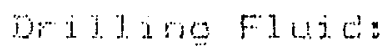

mellt,

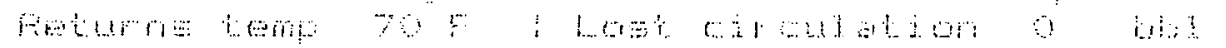

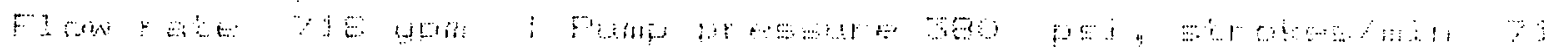

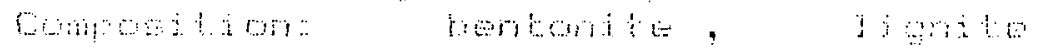

(1) : : : : :

b)

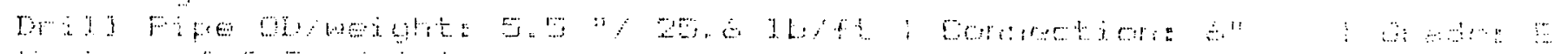

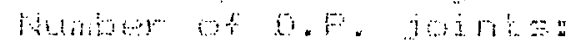

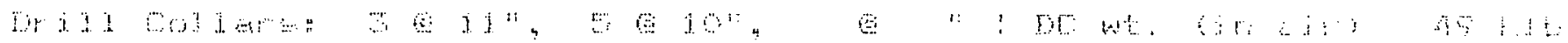

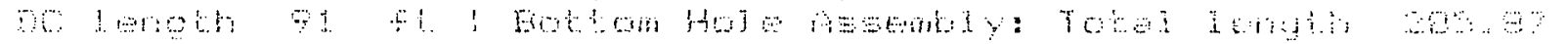

в

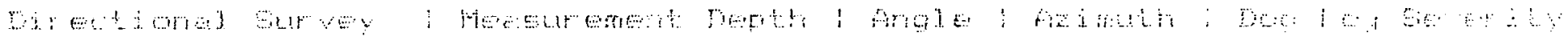

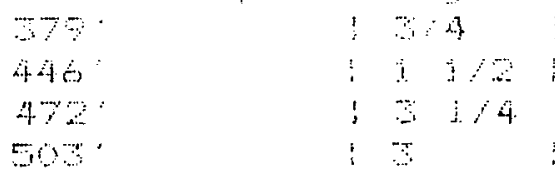

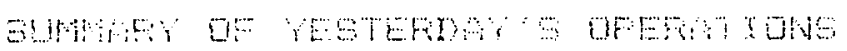

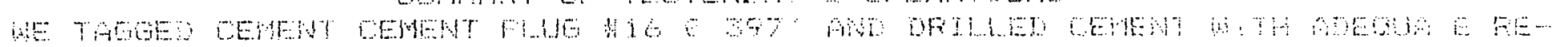

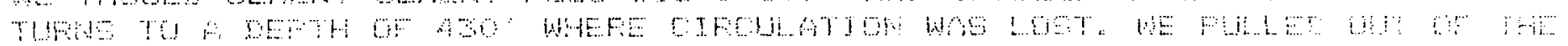

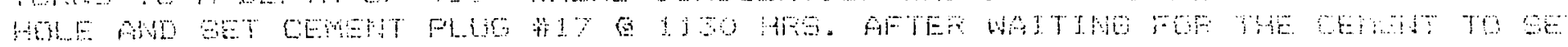

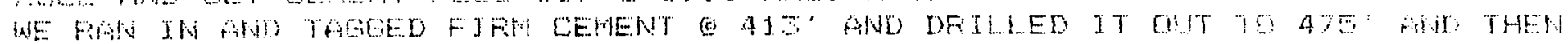

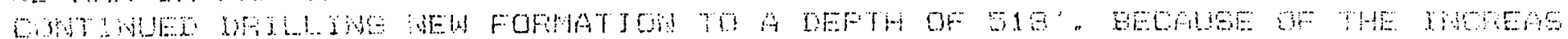

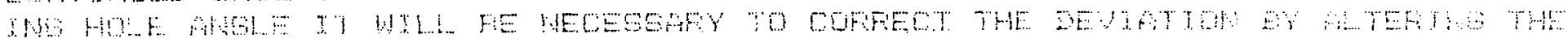

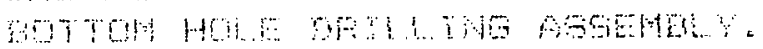

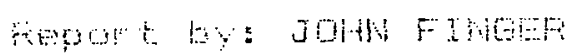


EPOCH HELL LOGGING DOE/SANDIA MAGMA ENERGY WELL LUF 51-20 DAILY MUD LQG FEPORT

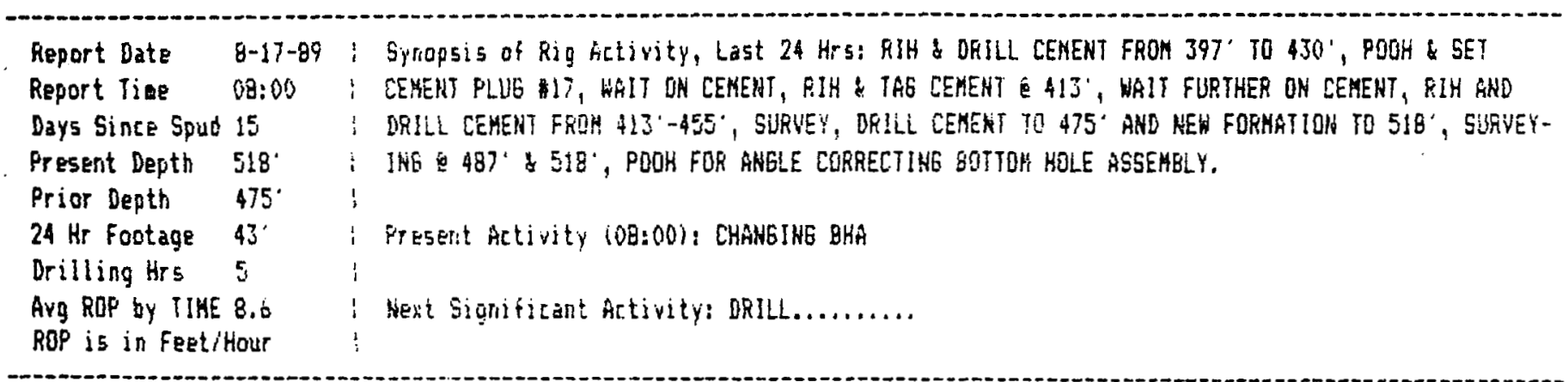

\section{DRILLIHG PARAMETERS AMALLYSIS}

\begin{tabular}{|c|c|c|c|c|c|c|}
\hline \multirow[b]{2}{*}{ ROP } & MAX & \multirow{2}{*}{$\begin{array}{l}\text { DEFTH } \\
476\end{array}$} & \multicolumn{2}{|c|}{ MIR E DEPTH } & \multicolumn{2}{|c|}{ AUERABE } \\
\hline & 59 & & 2 & $489^{\circ}$ & 15 & by RATE \\
\hline HOB & 18 & $476^{\circ}$ & 11 & $489^{\circ}$ & 15 & $k-1 b_{5}$ \\
\hline RPH & 74 & $500^{\circ}$ & 11 & $809^{\circ}$ & 58 & \\
\hline $\mathrm{RQ}$ & 29 & $477^{\circ}$ & 11 & $495^{\circ}$ & 17 & $d a$ \\
\hline r & 469 & $490^{\circ}$ & 234 & $518^{\circ}$ & 360 & PSI \\
\hline
\end{tabular}

MUD + GAS PARAMETERE AKALYSIS

\begin{tabular}{|c|c|c|c|c|c|c|}
\hline & MAXE & OEFTH & $H ! H$ & DEFTH & AUEAR & \\
\hline $\mathrm{CO} 2$ & 401 & $519^{\circ}$ & 327 & $49^{\circ}$ & 365 & $p p$ it \\
\hline CONB IN & 1132 & $500^{\circ}$ & 1011 & $513^{\prime}$ & 1111 & ppo $\mathrm{HaCl}$ \\
\hline COMD OUT & 1005 & $489^{\circ}$ & 504 & $513^{\prime}$ & 980 & DPA KaC] \\
\hline H2S & 0 & & 0 & & 0 & pes \\
\hline HYDC GAS & 0 & & 0 & & 0 & units \\
\hline$M H$ & 8.7 & $507^{\prime}$ & 8.6 & $480^{\circ}$ & 8.0 & Itig \\
\hline NH OUT & 8.7 & 518 & B. & $45^{\prime}$ & 8.6 & $16 / 9$ \\
\hline pH IN & 11.9 & $517^{\circ}$ & 11.0 & $476^{\circ}$ & 11.7 & \\
\hline pH OUT & 12.0 & 516 & 11.8 & $\left\{7^{\circ}\right.$ & 11.9 & \\
\hline TEMP IN & 72.1 & $493^{\circ}$ & 71 & $508^{\prime}$ & 72 & $\operatorname{deg} F$ \\
\hline TEMP OUT & $7 !$ & $494^{\prime}$ & 70 & $518^{\circ}$ & 71 & $\operatorname{deg} F$ \\
\hline
\end{tabular}

Conn Gas / Trip Gas i Methane: No Hydrocarbons

\section{LITHELOGY}

General Description: ISeg DIBIThL HUD LOG for details)

901-100\% RHYOL STE- LIGHT BROWWISH GEAY OVERALL WITH COHAON TO OCCASIONAL GRAYISH FINK BANDIHS AND DECREASING TUFFACEDUS IKTERLAMINATIONS, DECREASEO HOTTLING, HARD, MOD BFITTLE, COMFETENT. APKANITIC HITH RARE OUARTI PHENOCRVSTS, FIMELY SUCRDSIC TEXTURE, RGRE GLTERATIONS AND DEVIATRIFICATION.
Sample Luoging Conditions, other reaark5: EOOD SAMPLE RETURNS WITH CGMHOW LOST CIRCULATIOH HATERIAL ADDED TO PRESERVE RETURNS.

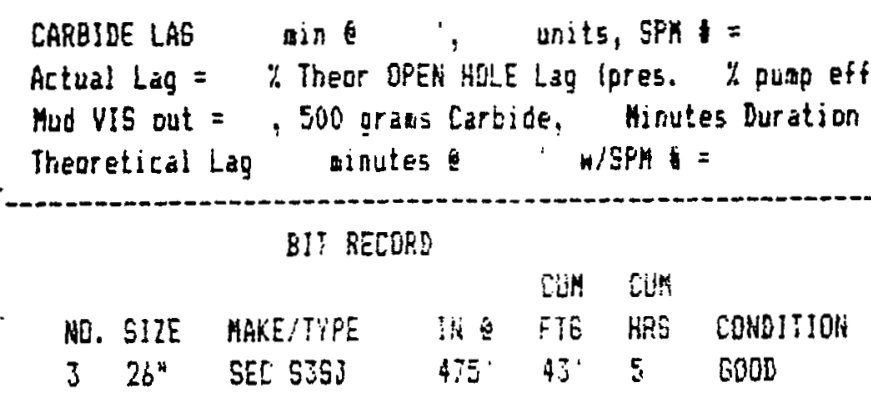

Report by: gILL GILMOUR
Sataple Shaker Screen Size: $1=40 / 60$ \& $12=40 / 60$ KESH Latest Screen Sjze Change or: 8-2-89 (Start of wel!?

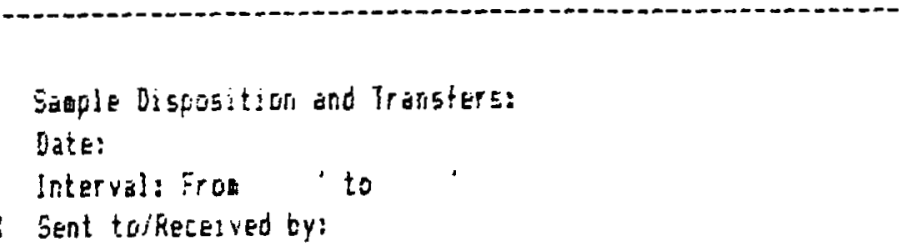




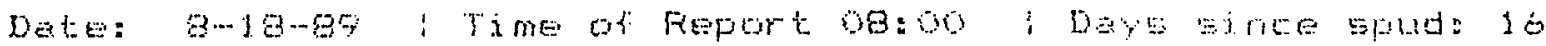

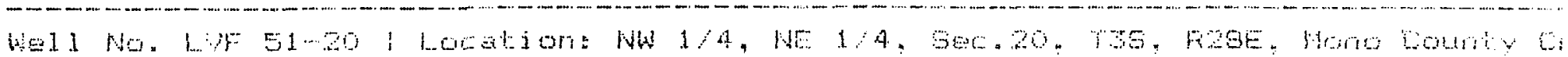

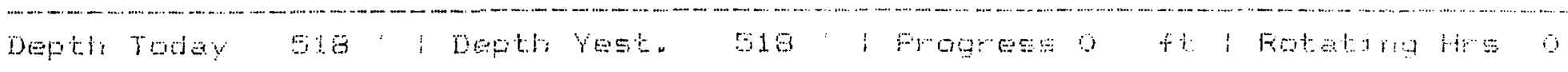

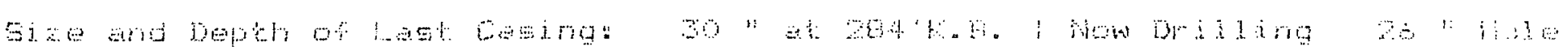

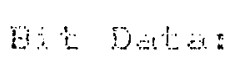

No :

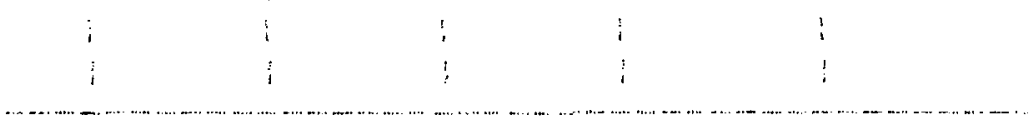

\begin{tabular}{|c|c|c|c|c|c|}
\hline 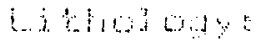 & $i$ & An+tकan & & $\dot{i}$ & Ob- \\
\hline & 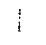 & $\ldots$ & +1 & $\vdots$ & \\
\hline & $\vdots$ & $\cdots$ & +1 & 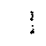 & \\
\hline & $i$ & $\ldots$. & 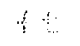 & $\vdots$ & \\
\hline & $\vdots$ & $\cdots$ & $+i$ & i & \\
\hline & $!$ & $\ldots .$. & 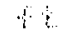 & & \\
\hline
\end{tabular}

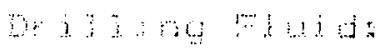

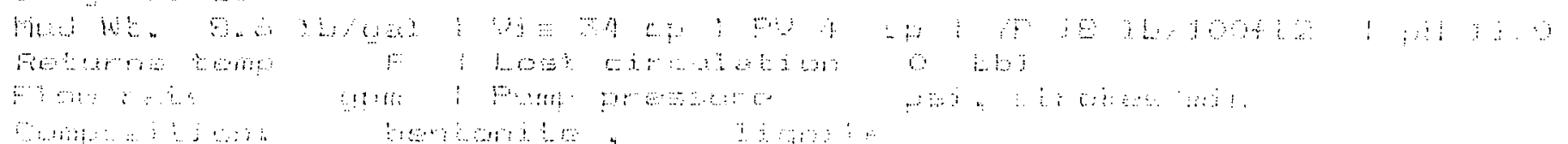

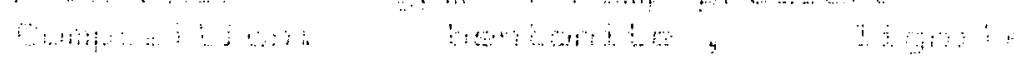

H.

\%

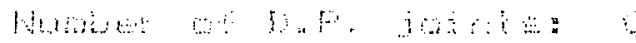

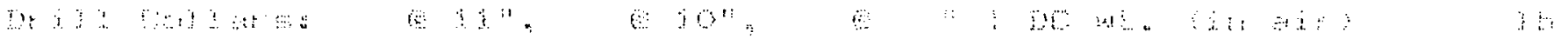

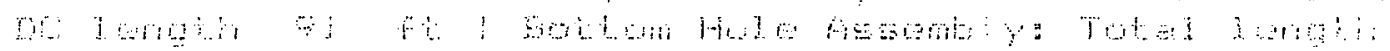

अ,

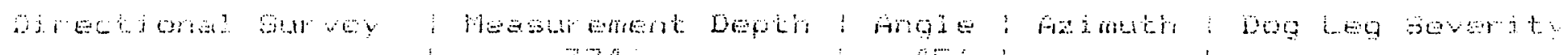

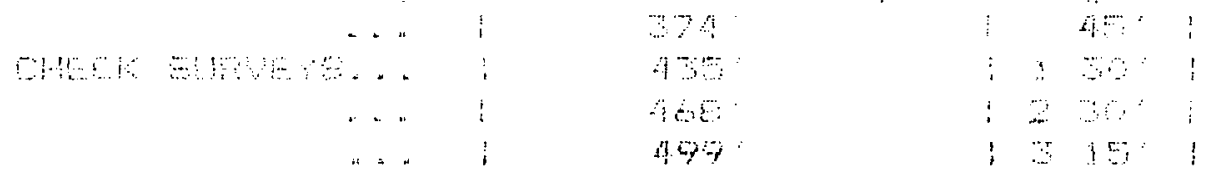

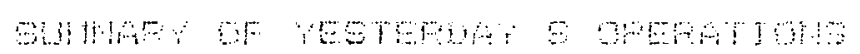

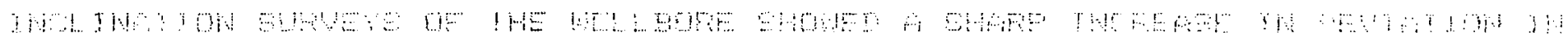

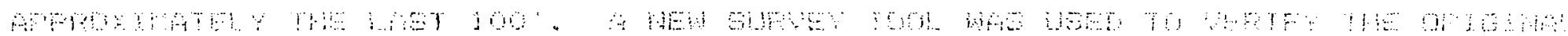

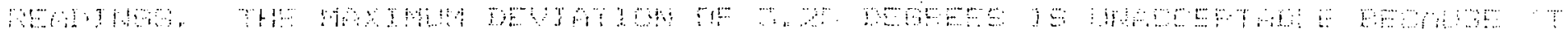

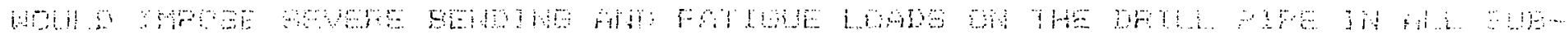

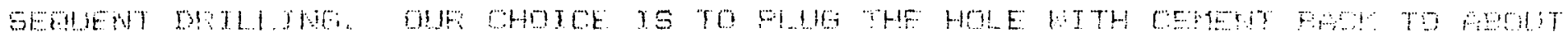

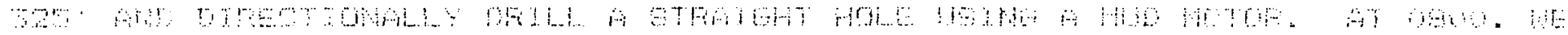

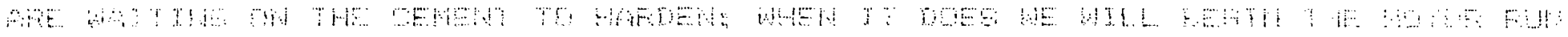


EFOISH WELL LOESIVE

DOE/SANDIA MAGMA ENERGY WELL LVF 51-20 DAILY MUD LOG REFDFT

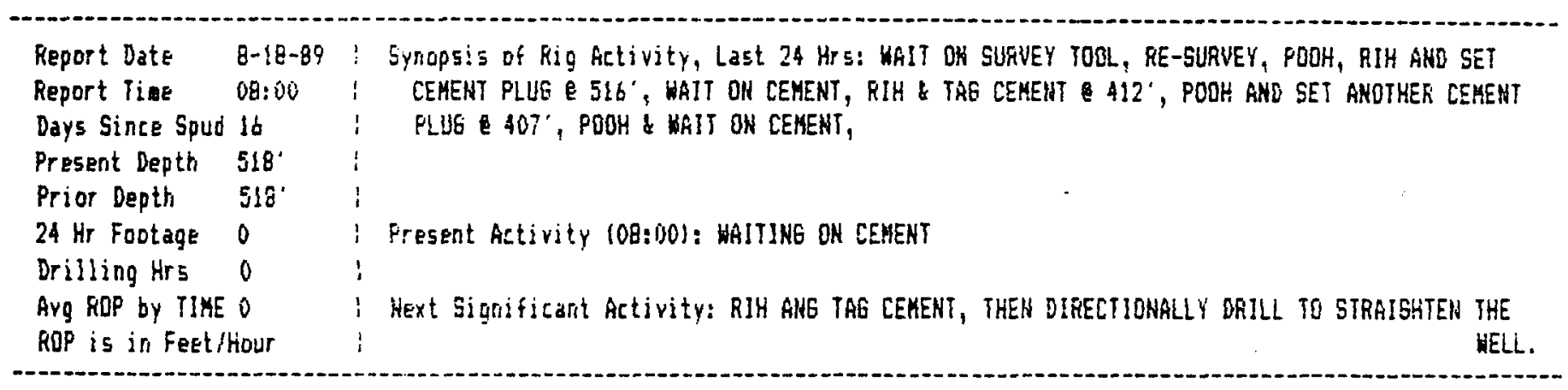

DRILLING PARAHETERS ANGLISIS

MEX DEPTH MIN E DEPTH HUERGEE

bY RATE

$x-1 b s$

HOS

RPH

TRQ

$p P$ aaps :

PS?

\section{LITHOLOGY}

General Descrjution: (See Dis!thi MUD LOG for details)

KO NEH SITHOLOAY DRILLEO

HUD + GHS FAEAHETEFS ANALYSIS

MAX E DEFTH MIN DEPTH AUERAGE

\section{$\mathrm{CO2}$}

COND IIN

COND OUT

H2S

HYDC GAS

KH IN

MH OUT

pH IH

pH OUT

TEMP IN

TEMP OUT pps $i$ ppo HaCl ! ppe HaC! i

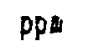
units $16 / 9$ lbig Sample Logging Conditions, other rearks:

Conn Gas / Trip Gas / Methare : ho Hyarocarbons

CREBIDE LAE WIIE, Units, SPY Z =

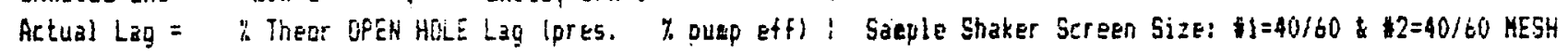
Hud VIS out $=$, 500 ạrans Carbide, Minutes Duration ! Theoretical Lag ginutes of 'w/SPM $=$ !

Theoretical Lag

BIT RECORO

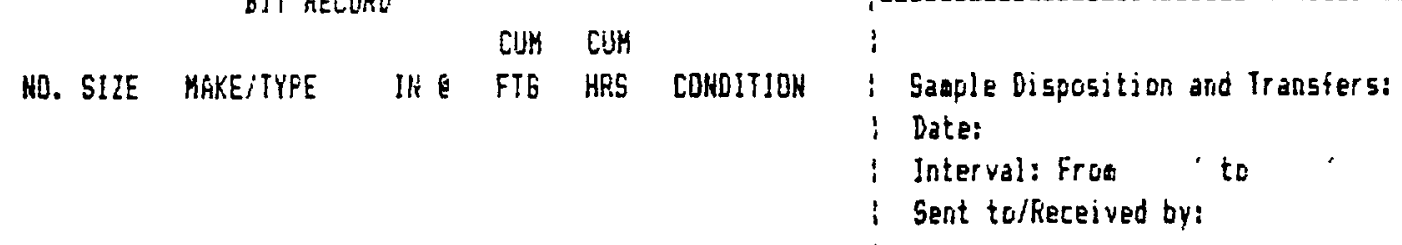

Report by: BILL GILMOUR

Litest Screen Size Change on: 8-2-89 (Start of well) 


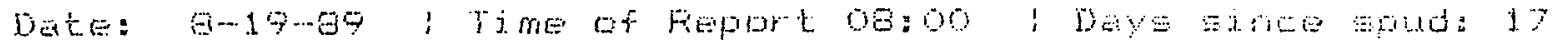

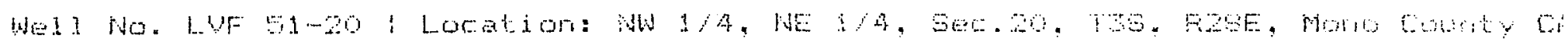

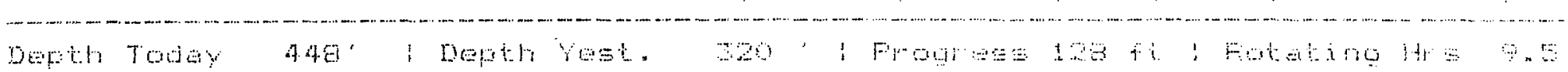
(1)

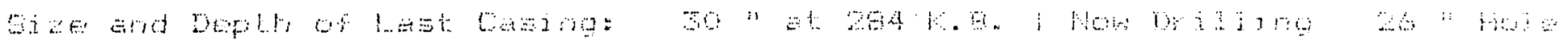

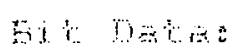

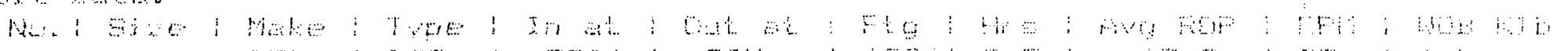
4 20 : i

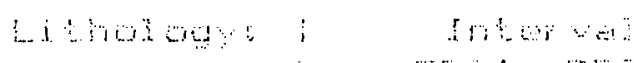
ar 40,490

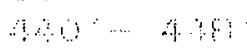

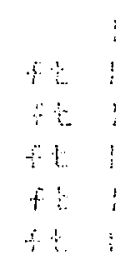

Doseratam

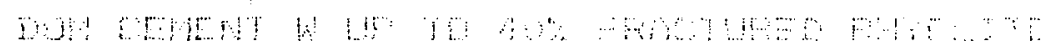

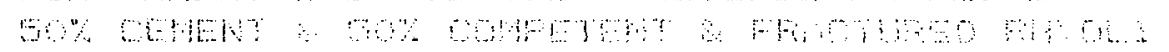

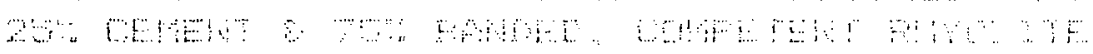

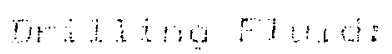

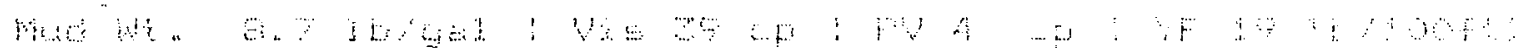

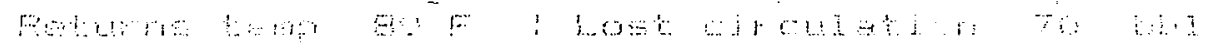

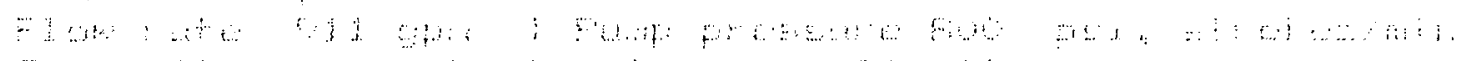

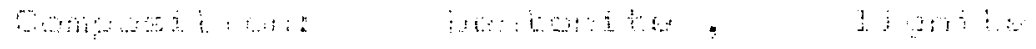

in: i: i: : :

I.

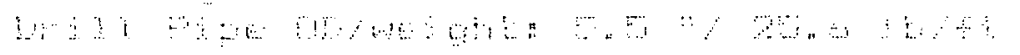

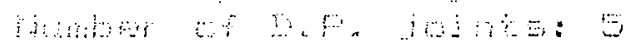

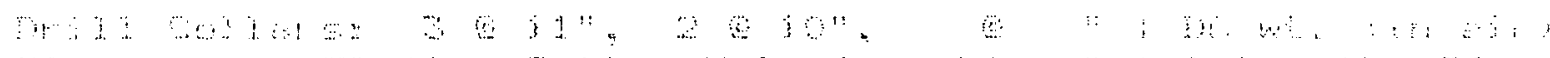

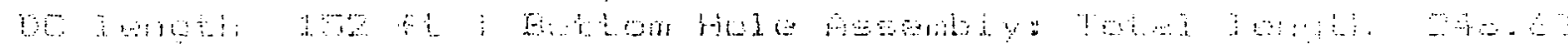

It

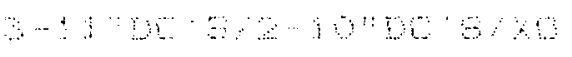

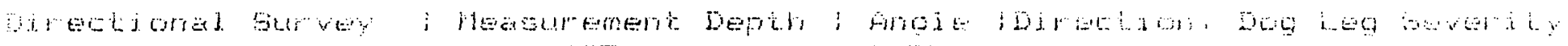

\begin{tabular}{|c|c|}
\hline 20 & 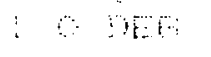 \\
\hline 80 & 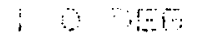 \\
\hline 98 & $\begin{array}{lll}1 & 0 & = \\
i & & \end{array}$ \\
\hline
\end{tabular}

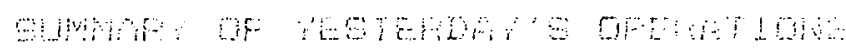

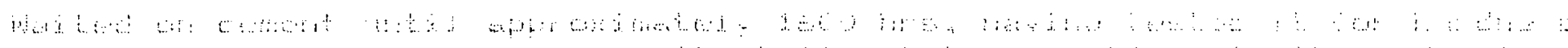

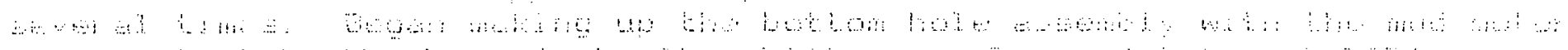

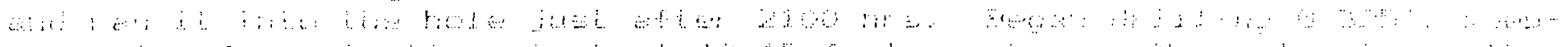

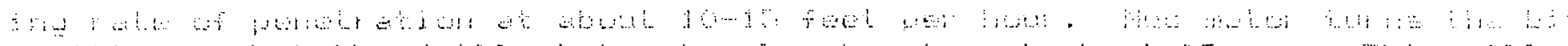

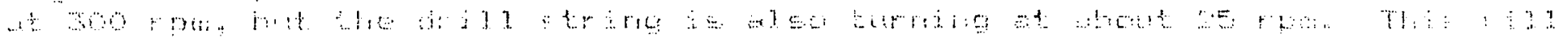

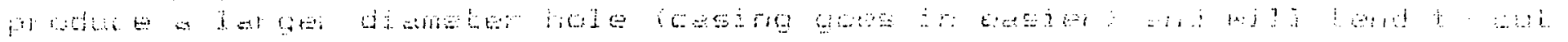
औ..

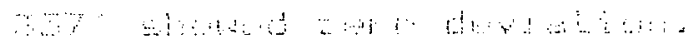


EFOGH WEL $10 B G I N G$

DOE/SANDIA MAGMA ENEFGY WELL LUF 51-20 DAILY MUD LOG REPORT

\begin{tabular}{|c|c|c|c|}
\hline $\begin{array}{l}\text { Report Date } \\
\text { Report Tine }\end{array}$ & $\begin{array}{l}8-19-89 \\
08: 00\end{array}$ & $i$ & $\begin{array}{l}\text { Syropsis of Rig Activity, Last } 24 \text { Hrs: WAIT ON CEMERT, RIH \& TAG CEMENT } 310^{\circ} \text {, POLISH OFF } \\
\text { TO } 315^{\circ} \text {, WAIT OH CEMENT, POLISH OFF TO } 319^{\circ} \text {, POOH, MIU HUD MOTOR BHA, DIRECTIONALLY ORILL }\end{array}$ \\
\hline Days Since Spud & 17 & ! & FROM $319^{\circ}$ TO $448^{\circ}$, SURVEYING $325^{\circ}$ \& $355^{\circ}$ \\
\hline Present Depth & $448^{\circ}$ & $!$ & \\
\hline Prior Depth & $320^{\circ}$ & ! & \\
\hline $24 \mathrm{Hr}$ Footage & 128 & $!$ & Present Activity (08:00): EIRCULATE FOR A SURVEY \\
\hline Drilling Hrs & 9.5 & ! & \\
\hline Avg ROP by TIME & 13.5 & $\vdots$ & Kext Significant hetivity: COKTINUE DAULLING \\
\hline ROP is in Feetil & Hour & $:$ & \\
\hline
\end{tabular}

\section{DRILLING PARAMETERS ANALYSIS}

\begin{tabular}{|c|c|c|c|c|c|c|}
\hline & MAX E & DEPTH & HIS & DEPTH & \multicolumn{2}{|c|}{ AVERAEE } \\
\hline ROP & 62 & $321^{\circ}$ & 4 & $375^{\circ}$ & 19 & by RATTE \\
\hline NOB & 13 & $346^{\circ}$ & 2 & $433^{\prime}$ & 6 & $k-1$ bs \\
\hline RPH & 26 & $409^{\circ}$ & 24 & $4 ! 2^{2}$ & 25 & \\
\hline TRB & 14 & $333^{\circ}$ & 3 & IE9 & 8 & amps \\
\hline$P P$ & 1073 & $347^{\circ}$ & 299 & $386^{\circ}$ & 821 & PSI \\
\hline
\end{tabular}

\section{WUS + GAS PARAKETERS AIIALVIS}

$\begin{array}{lll} & \text { MAX Q OEPTH } \\ \text { CO2 } & 557 & 409^{\circ} \\ \text { COND IN } & 3760 & 414^{\prime} \\ \text { COND OUT } & 2924 & 393^{\circ} \\ \text { H2S } & 0 & \\ \text { HYDC GAS } & 0 & \\ \text { HH IN } & 8.6 & \\ \text { HW OUT } & 8.7 & 345^{\circ} \\ \text { PH IN } & 12.0 & 432^{\prime} \\ \text { PH OUT } & 12.1 & 320^{\circ} \\ \text { TEMP IN } & 80 & 414^{\prime} \\ \text { TEME OUT } & 77 & 419^{\circ}\end{array}$

\section{MIN O DEPTH AUERAGE}

$314329^{\circ} 456 \mathrm{ppE}$

$3012327^{\circ} 3439 \mathrm{pph} \mathrm{HaCl}$ !

2429 333' 2715 ppa NaCl !

0

i.

E.e $\quad$ Q.6 lbig

S.5 414 8.6 1big

$11.8522^{\circ} \quad 11.9$

$11.8 \quad 424.11 .9$

$75 \quad 33$

\section{LITHOLOGY}

Genera! Description: (See DIGITAL MUD LOG for detaijs) RHYOLITE - PALE RED TO LT BRIISH GRAY $~ O C C$ REODISH ORAMGE MOITLING ANG BANOING, MOD HARD, APHANITIC, WITH OCC GUARTIITIC INTERLAKS BOUNDED BY REDOISH ORAKGE K-FELDSPAR BAKDS, RARRE QULARTI PHENOCRYSTS, OCC PARTJALLY OEYJTRIFIED, COHHON FRACTURES WITH IRON OXIDE STAINING WITHIN THE UPPER ORILLEO INTERVAL DECREASING HITH DEPTH.

40\% FORHATIOH $380^{\circ}$

50\% FORARTIOH \& $400^{\circ}-440^{\circ}$

75T FORMATION $448^{\circ}$

Conn Gas / Trap Gas / Hethone : ho Hydrucartions

CARBIDE LAG ain E : units, SPH $1=$

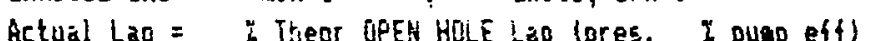
hud VIS out $=, 500$ grans Carbide, Minutes Duration Theoretical Laq ainutes $\$$ BIT RECORD

\begin{tabular}{|c|c|c|c|c|c|}
\hline \multirow[b]{3}{*}{ HO. SIZE } & \multicolumn{2}{|c|}{ BIT RECORD } & \multirow{3}{*}{$\begin{array}{l}\text { Cot } \\
\text { FTE }\end{array}$} & \multirow[b]{2}{*}{ CUH } & \multirow[b]{3}{*}{ COHOSTION } \\
\hline & & & & & \\
\hline & MAXE/TYPE & IN & & HRS & \\
\hline $426^{\circ}$ & STE 235 & $320^{\circ}$ & $128^{\circ}$ & 4.5 & SIH \\
\hline
\end{tabular}

Report Ly: BILL GILMOLR
Sample Shaker Screen Size: 1$]=40 / 60 \& 12=40 / 60 \mathrm{KESH}$ Latest Screen Size Change on: 8-2-89 (Start of well) 
Dete: 8-21-69 i Time of Report 08:00 : Days mnce soud 19

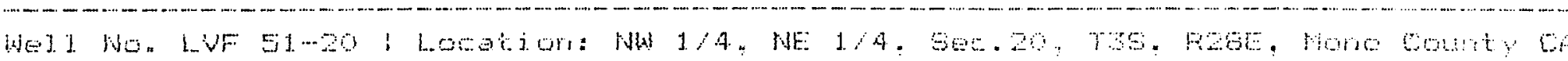

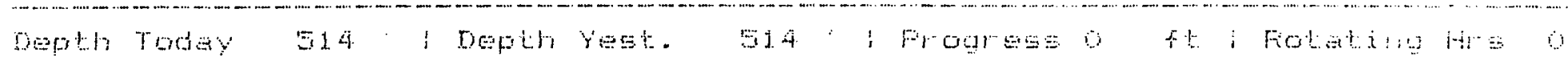

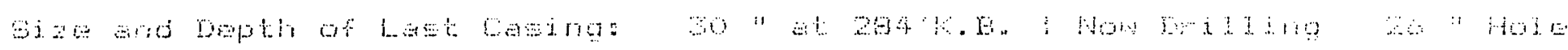

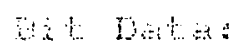

He. $\begin{array}{cccccccc}\vdots & \vdots & \vdots & \vdots & \vdots & \vdots & \vdots & \vdots \\ \vdots & \vdots & \vdots & 1 & \vdots & \vdots & \vdots & \vdots \\ \vdots & \vdots & \vdots & \vdots & i & \vdots & \vdots & \vdots\end{array}$

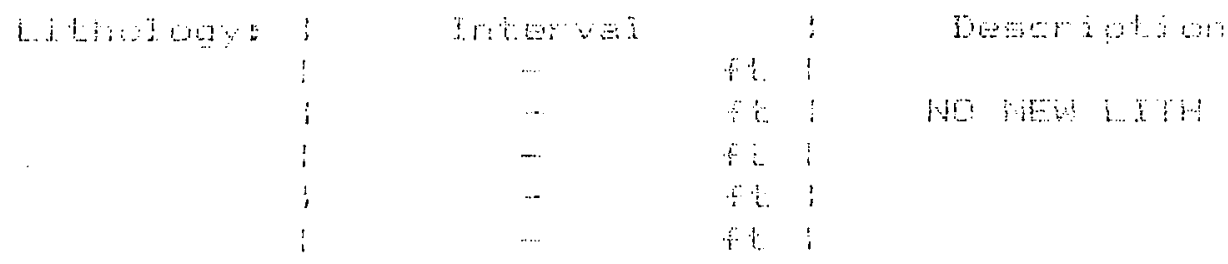

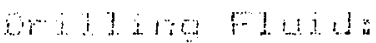

mat H.

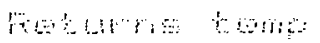

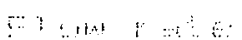

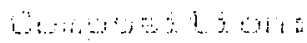

1.:

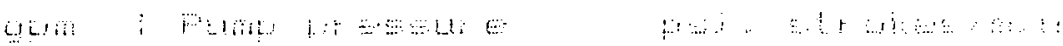

: $: 1+\ldots$

:

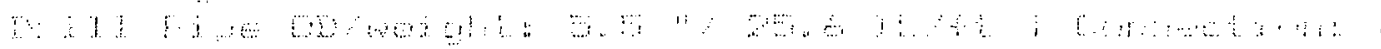

(:) : : : : : :

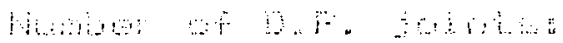

Y

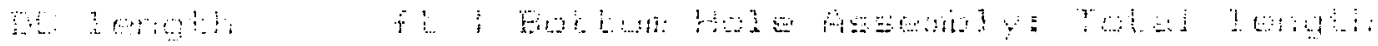

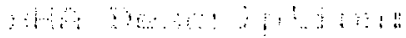

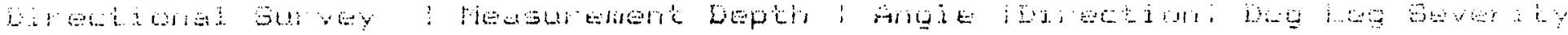

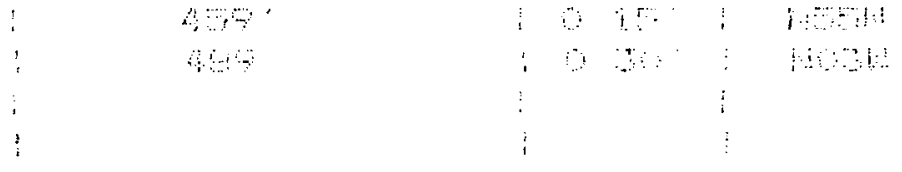

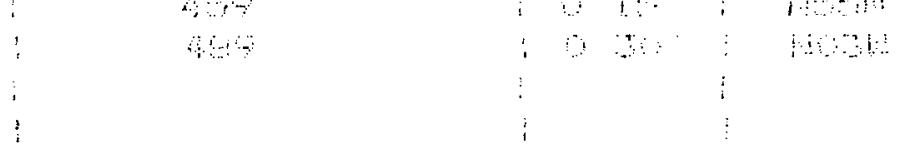

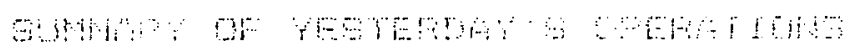

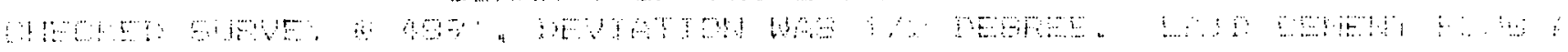

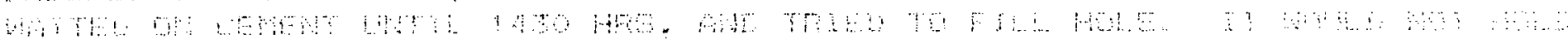
:...

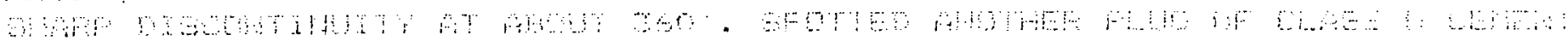

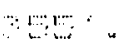

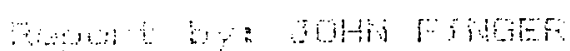




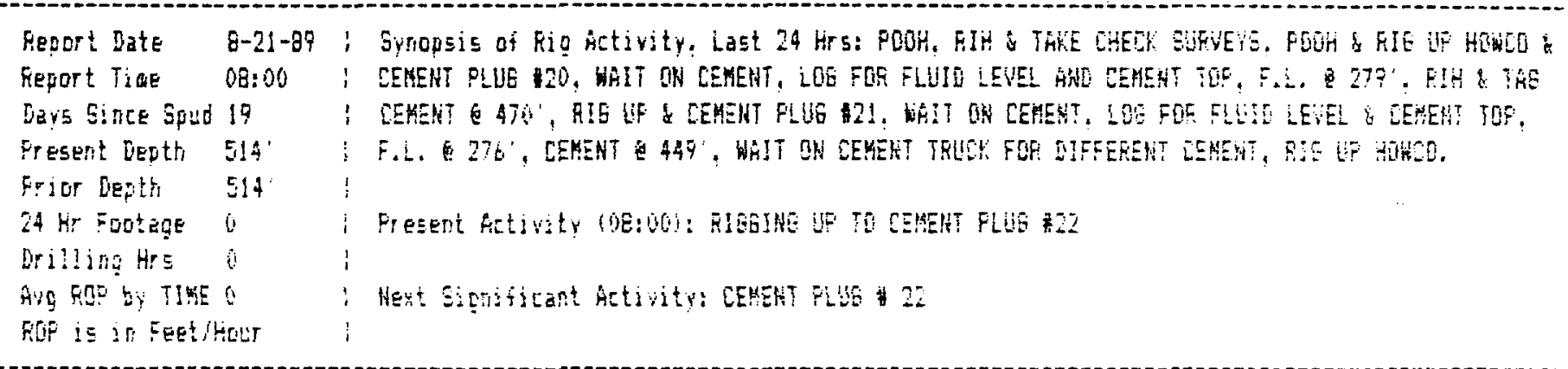

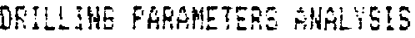

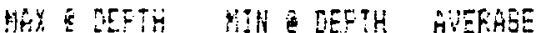

BOF
HES
BEP
THE
OF

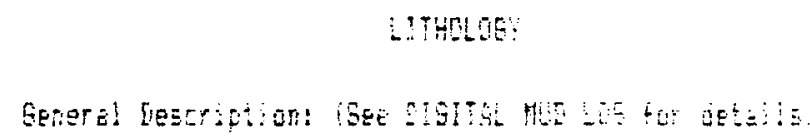

ats

(i) ? ?

Cont wit

His

Hot Gis

Mt: 1 in

Mi: 015

y.tis

p)

TEHE IH

TEYH D:Ti
E. TATE

$1-1,5$

atps

Fi!
WH WEFTH HIS DEFTH BUEGEE

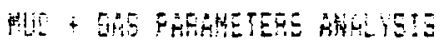 \\ -}

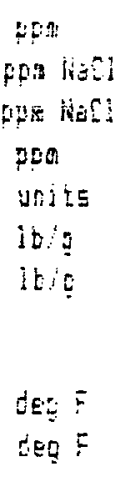

$$
\text { . }
$$




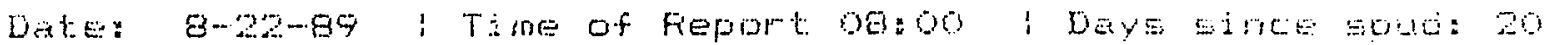

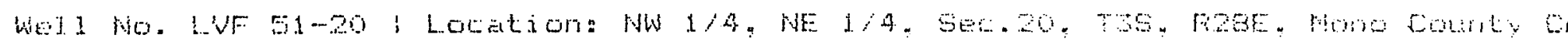

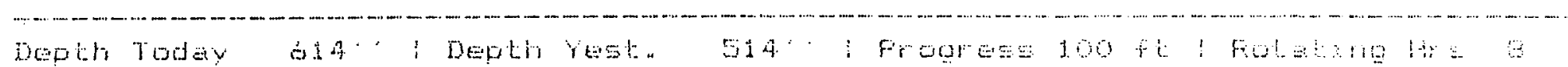
D.

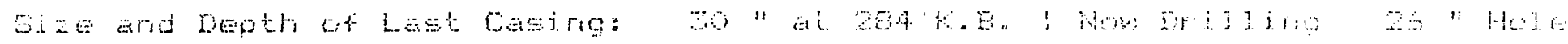
at bat

Wo: 5a

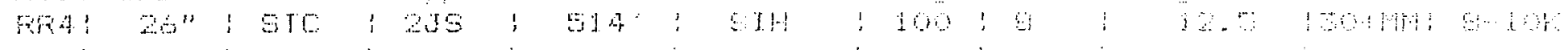

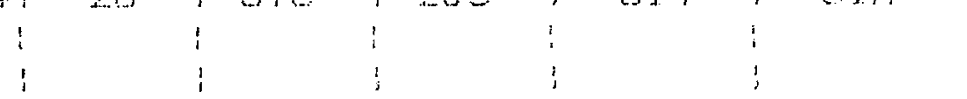

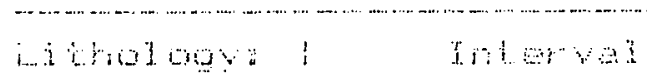
: 53 का

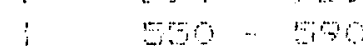
$50-610$

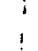

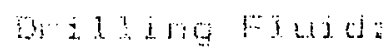

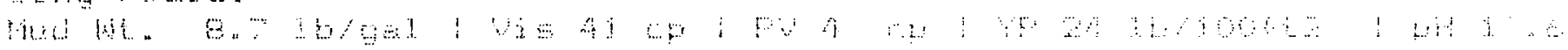

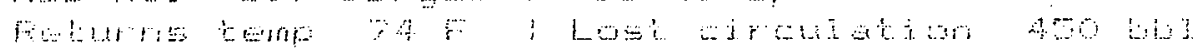

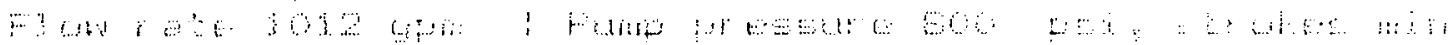

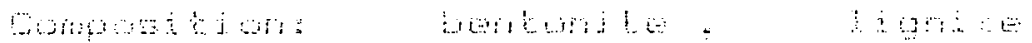
otris:

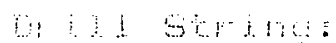

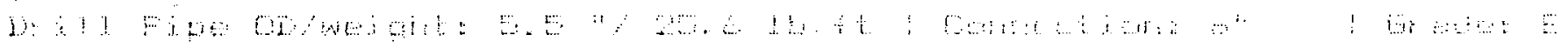

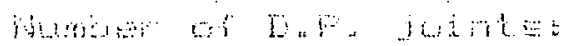

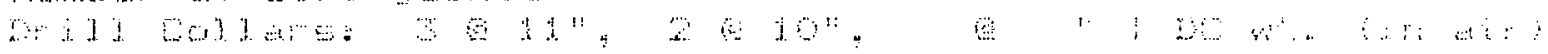

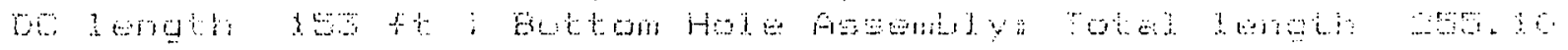

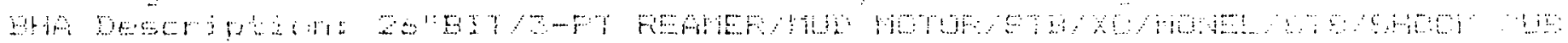

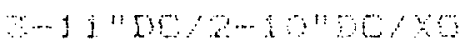

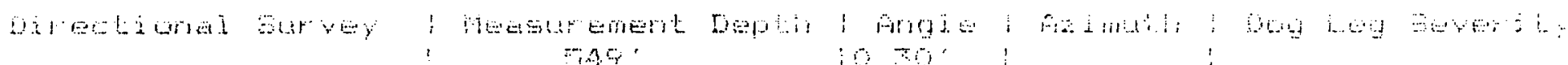

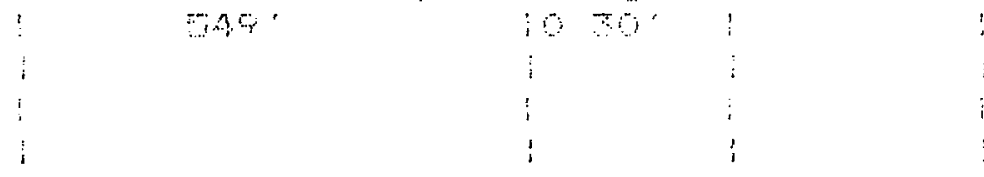

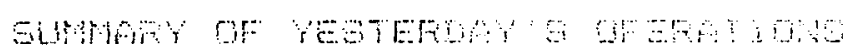

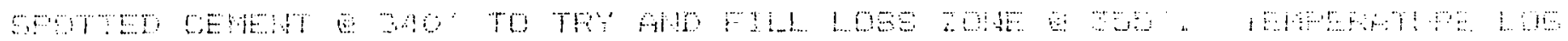

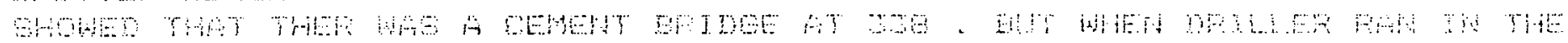

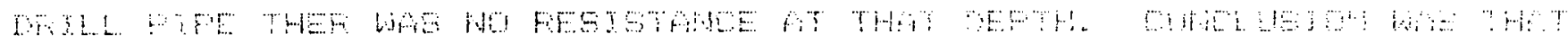

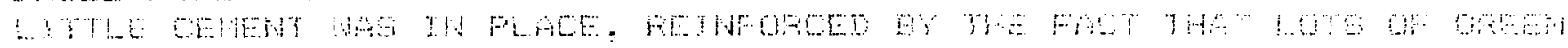

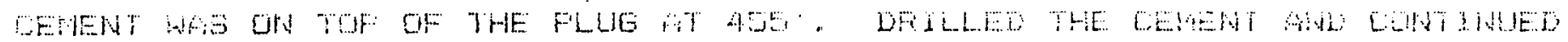

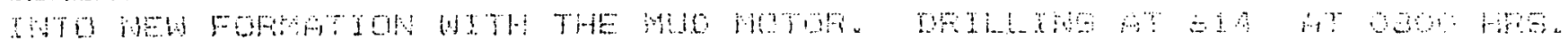




\section{EPOCH WELL LDEGIHG}

\section{DOE/SANDIA MAGMA ENERGY WELL LUF 51-20 DAIL'Y MUD LDG FEFORT}

\begin{tabular}{|c|c|c|}
\hline Geport Date & $8-22-89$ & 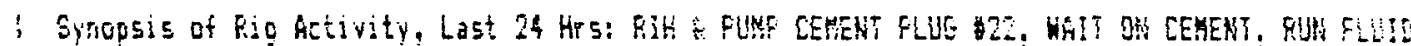 \\
\hline Report Tine & $08: 00$ & 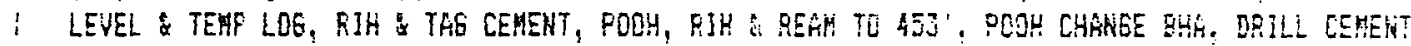 \\
\hline Days Since Spud & 20 & 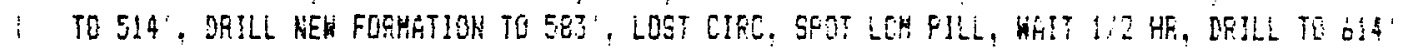 \\
\hline Frior bepth & $5 ! 4^{\circ}$ & $\vdots$ \\
\hline $24 \mathrm{Hr}$ Sactage & 100 & 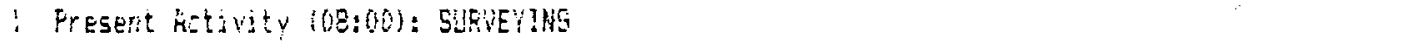 \\
\hline Driliog irs & $E$ & 1 \\
\hline WAT :S :H FEEL & How? & $\frac{1}{i}$ \\
\hline
\end{tabular}

\section{DOLLIUE FARAMETERE AHALIVIS}

\begin{tabular}{|c|c|c|c|c|c|c|}
\hline & $114: 8$ & EETH & mIh & EETH & \multicolumn{2}{|c|}{ AUEFAGE } \\
\hline Ear & 40 & $55^{\circ}$ & 3 & $5 E^{\prime}$ & 17 & by RATE \\
\hline WiE & 53 & 54 & 4 & $552^{\circ}$ & 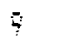 & $k-j+5$ \\
\hline EPF: & 50 & 55 & $2 E$ & res: & 29 & \\
\hline THE & 12 & 5,2 & 4 & $520^{\circ}$ & $\hat{y}$ & anps \\
\hline F & 105 & $550^{\prime}$ & 55 & $523^{\circ}$ & 907 & FS! \\
\hline
\end{tabular}

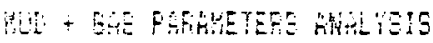

\begin{tabular}{|c|c|c|c|c|c|c|}
\hline & PA & BEFTH & 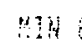 & DEETH & MES & \\
\hline 8 & $F$ & $5 \%$ & 0 & 575 & 34 & pef: \\
\hline 1: & $25: 0$ & 5 & 25 & $E:$ & 7455 & Eutit Mec! \\
\hline OtiE OJ & 265 & $540^{\circ}$ & 0 & $5 \mathrm{E}$ & 1967 & GER Kabj \\
\hline 28 & 8 & & i & & 0 & tofta \\
\hline 6is res & 0 & & 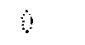 & & i & units \\
\hline in & E.? & 50 & 5.6 & 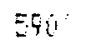 & E. ? & bia \\
\hline rib & E. & $5 ! 1$ & 5.5 & $510^{\circ}$ & 3.7 & itiog \\
\hline $0+2$ & $1 \therefore \therefore$ & $52 !^{\circ}$ & : : I. & 505 & 11.8 & \\
\hline pit no: & $1 ! .9$ & 515 & 11.7 & $570^{\circ}$ & 11.8 & \\
\hline 偝 J & TB & EES & 75 & 515 & 77 & DEQ $F$ \\
\hline TEHE DUT & 77 & $5:=$ & $7 i$ & 515 & 70 & deg F \\
\hline
\end{tabular}

\section{LITHE:}

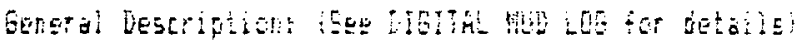

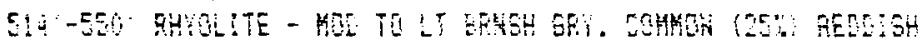

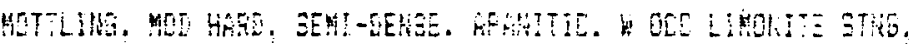

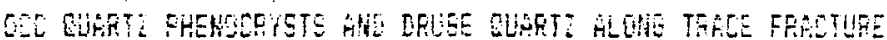

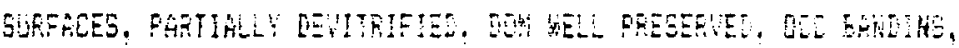
TP EWTITE IHGLSUKO.

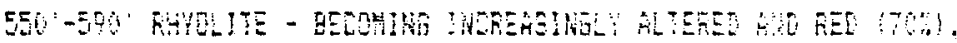

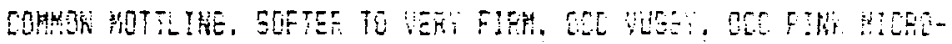

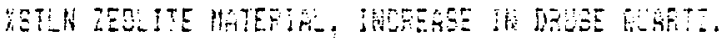

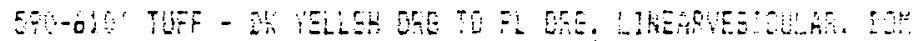

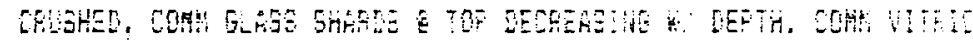

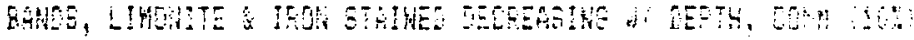

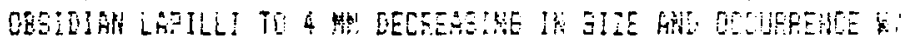
DETS.

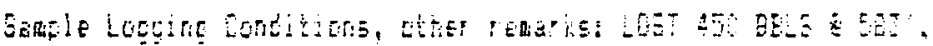

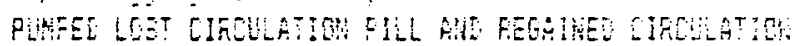

Cont Bja: Trit Bes Methane : Ho Hydratarbone

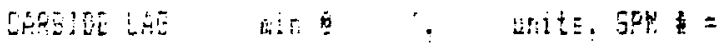

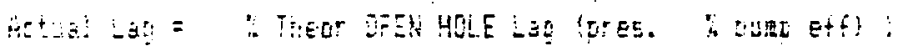
We

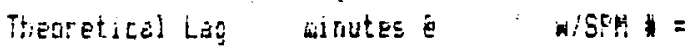

EIT BECORT

\begin{tabular}{|c|c|c|c|c|c|}
\hline & & & $\mathrm{COM}$ & Ebik & \\
\hline HE SUE & MBETYE & $\ln 6$ & FTE & HES & Seko: Jjuk \\
\hline $15420^{\circ}$ & 315215 & 5 & it: & $E$ & $\mathrm{SiH}$ \\
\hline
\end{tabular}

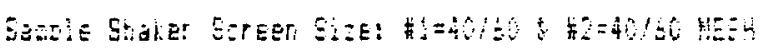

Latest Sereeri Size Challege on: \&-2-8? (5tart ji weil) 


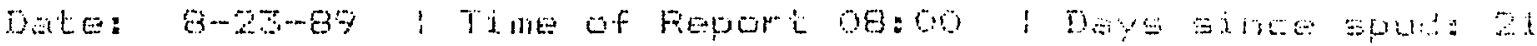

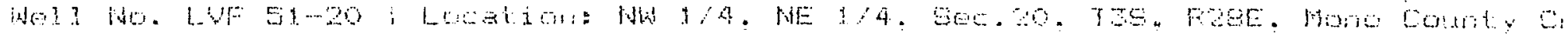

owp!r 7od उ. Bat

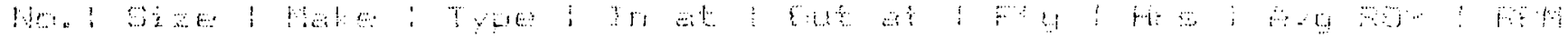

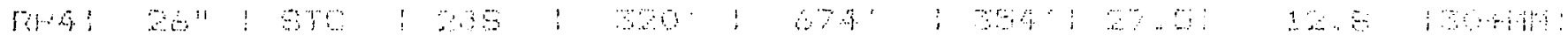
i

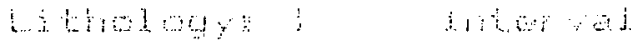
ij:

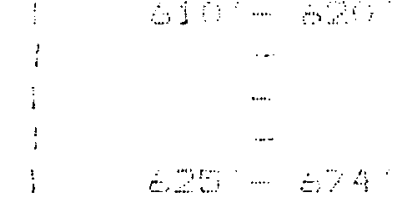

ti $\quad$ : br

1

bi y.

$\because \quad: \quad$ : $F\lfloor$ LI

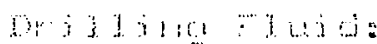

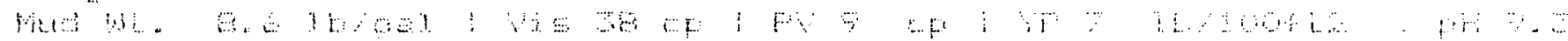

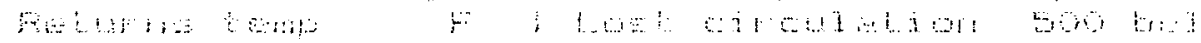

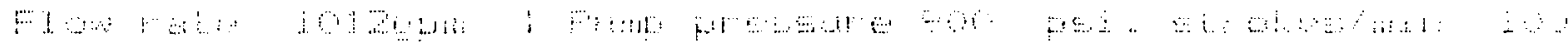

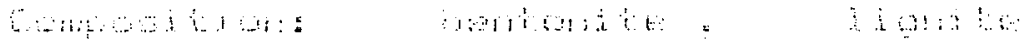

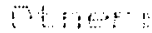

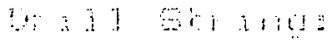

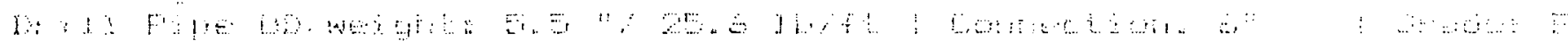

Hinal

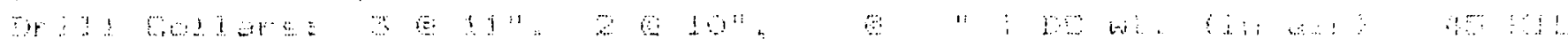

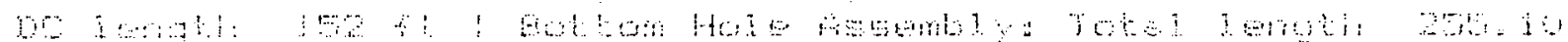

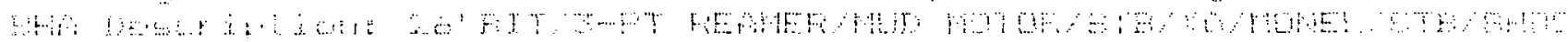

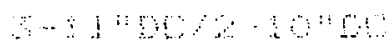

bi

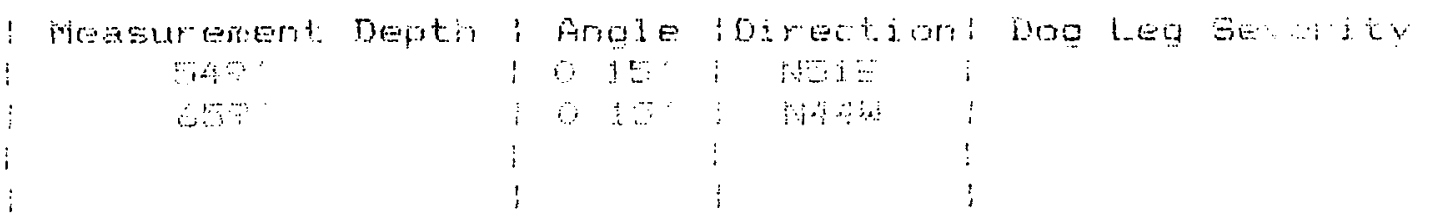

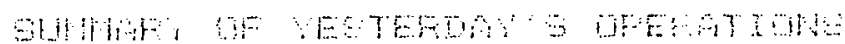

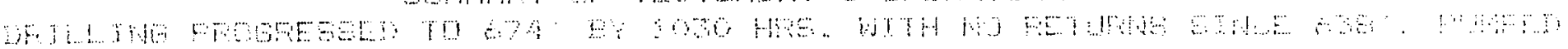

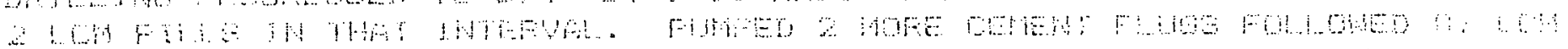

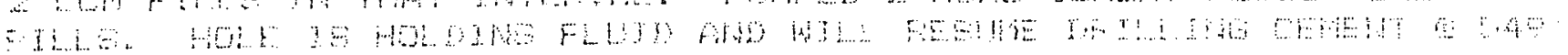

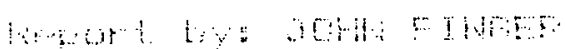


EPECS WELL LUGGING

DOE/SANDIA MAGMA ENERGY WELL LUF 51-20 DAILY MLID LDG FEFDFT

\begin{tabular}{|c|c|c|c|}
\hline Feport Bate & $8-23-8$ & ; & 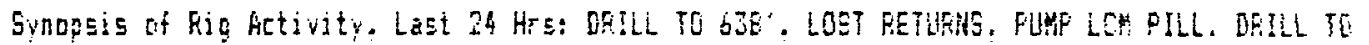 \\
\hline Feport Time & $09: 00$ & $\begin{array}{l}\vdots \\
\vdots\end{array}$ & 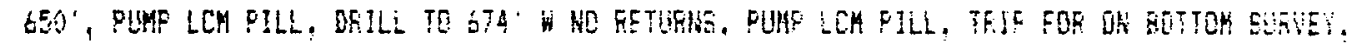 \\
\hline Days Since Spud & $2 !$ & ? & 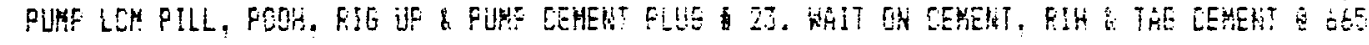 \\
\hline Present Desth & $674^{\circ}$ & : & 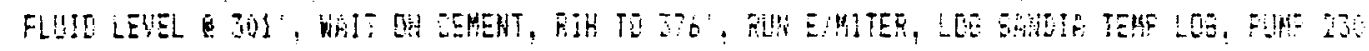 \\
\hline Prior Depth & $6 ! 4:$ & ; & 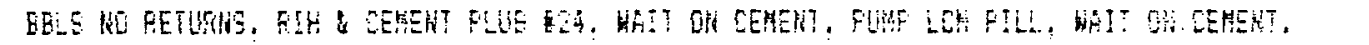 \\
\hline 24 Hr Footage & 50 & $\vdots$ & 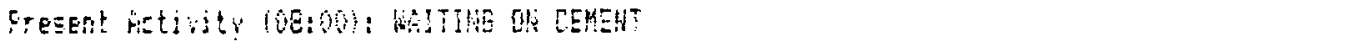 \\
\hline Dtallito itr & 2.5 & $i$ & \\
\hline fing Goe t: TIME & 24 & ! & 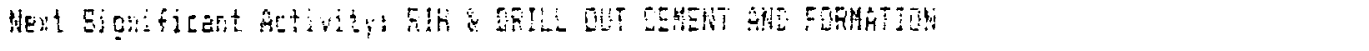 \\
\hline hot is in feth & & & \\
\hline
\end{tabular}

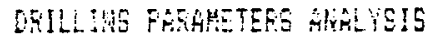

\begin{tabular}{|c|c|c|c|c|}
\hline MAY E JETH & y1s & DEFTH & SUES & AEE \\
\hline 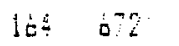 & 7 & $\dot{s}_{i} !$ & Ec & b) FEIE \\
\hline $52 \%$ & 2 & 43 & 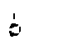 & $1-\frac{1}{2}=$ \\
\hline $07:$ & is: & b5: & 30 & \\
\hline 65 & $\dot{\varepsilon}$ & $85^{\circ}$ & 1 & 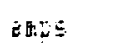 \\
\hline $159 \div 08$ & 170 & 040 & 1240 & $F E$ \\
\hline
\end{tabular}

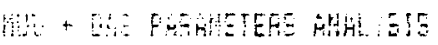

\begin{tabular}{|c|c|c|c|c|c|c|}
\hline & He: & resti & $n$ & PES:H & \multicolumn{2}{|c|}{ MEFEE } \\
\hline$[5$ & $\because=4$ & 517 & 8 & $=7$ & 35 & to: \\
\hline Ohe It & 125 & 25 & 179 & E & $5+8$ & yot Nâ \\
\hline crote of & 441 & $\therefore$ & $\theta$ & 58 & 5 & Fen lasis \\
\hline 498 & 8 & & 0 & & $\because$ & $p+4$ \\
\hline Hit no & $\because$ & & 0 & & 0 & Write \\
\hline sif It: & 5.7 & $8: 5$ & g.t & 675 & E.: & 10 \\
\hline$\because x$ & E.E & 2 & $\therefore$ & $674^{\circ}$ & 5.7 & 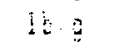 \\
\hline$p=14$ & 1.7 & bit & $1 \ldots 1$ & 6.94 & 11.5 & \\
\hline cH OHT & $1 ! .8$ & $6 ! 7$ & 6 & $674^{\circ}$ & 11.6 & \\
\hline 证等 & $\because$ & 53 & $i$ & 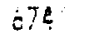 & 75 & des? \\
\hline That & 73 & 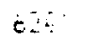 & 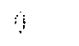 & 5 & 22 & deg \\
\hline
\end{tabular}

\section{$1140 \div 89$}

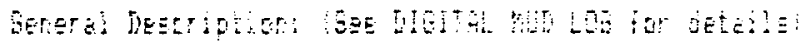

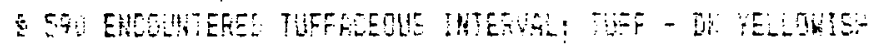

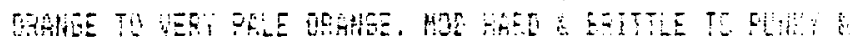

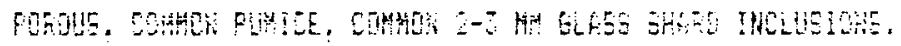

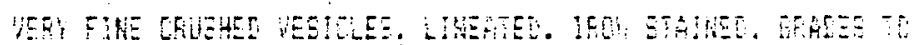

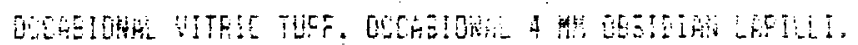

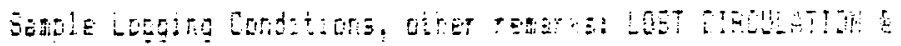

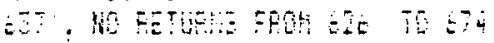

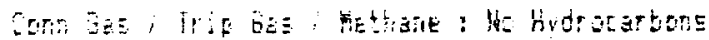

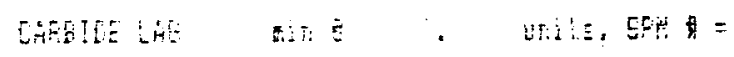

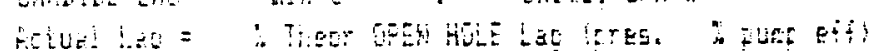

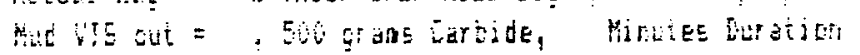

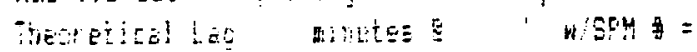

E: SECOSL

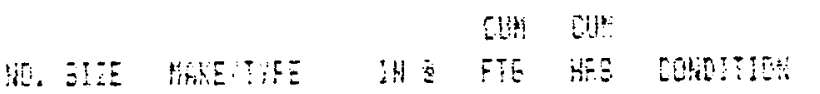

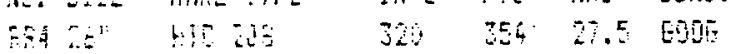

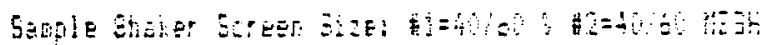

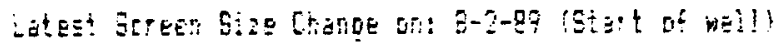

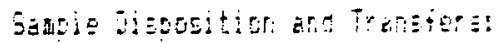

Dete:

literial: Syat in

Sert tofrered wed toi 


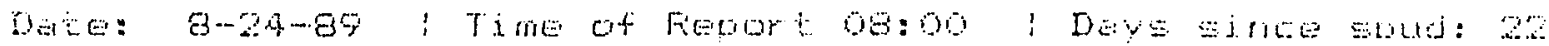

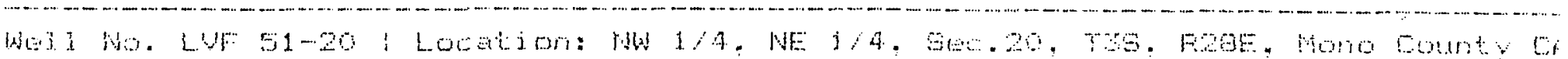

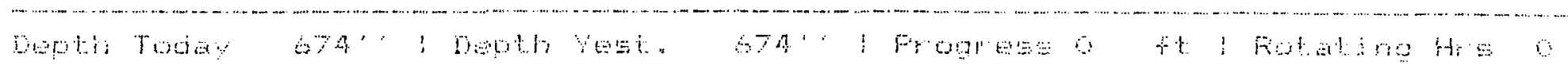

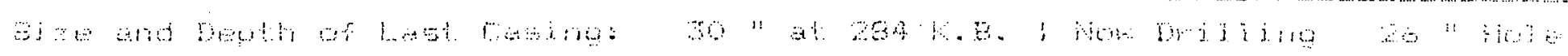

H.

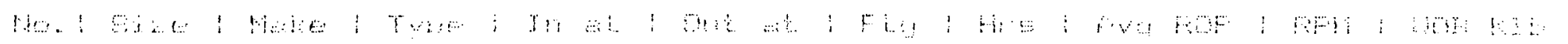

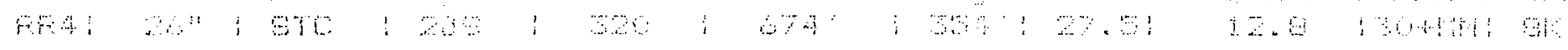

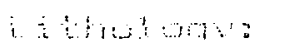

,

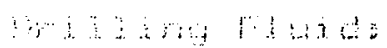
Hil

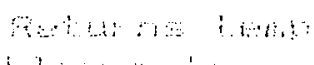

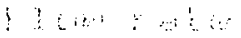
a.
(:) : : a

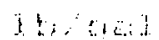

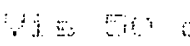

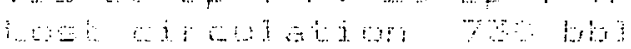
an

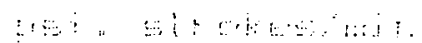

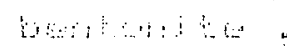
i.

1

M

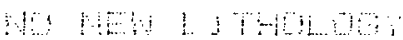

$\begin{array}{ll} & \vdots \\ 1 & \vdots \\ \ddots & \vdots \\ \ddots \vdots & \vdots \\ 1 & \vdots \\ \therefore & \vdots\end{array}$

:

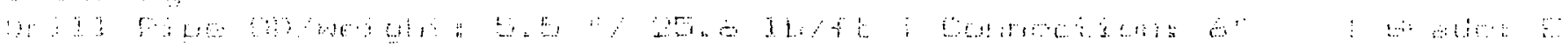

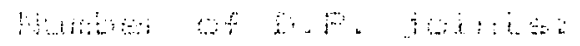

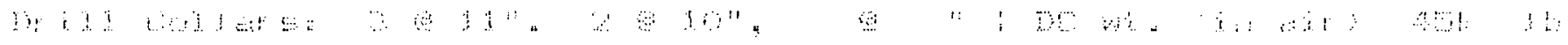

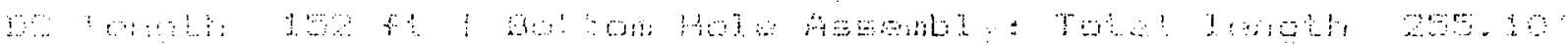

Al: a

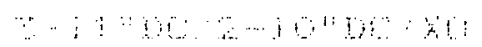

n

a if

\#!

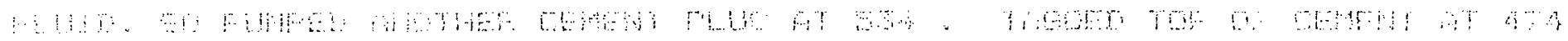

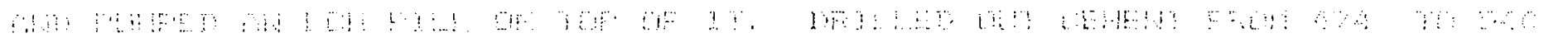

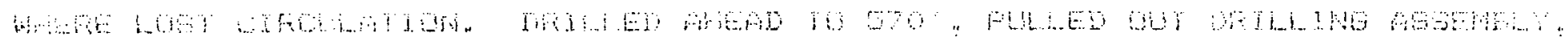

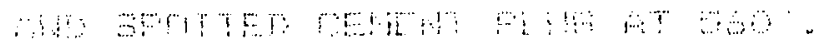

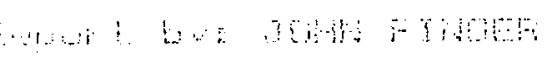


EPOCH HELL LOGETHG

\section{DOE/SANDIA MAGMA EINERGY WELL LUF 51-20 DAILY MUD LOG REFDRT}

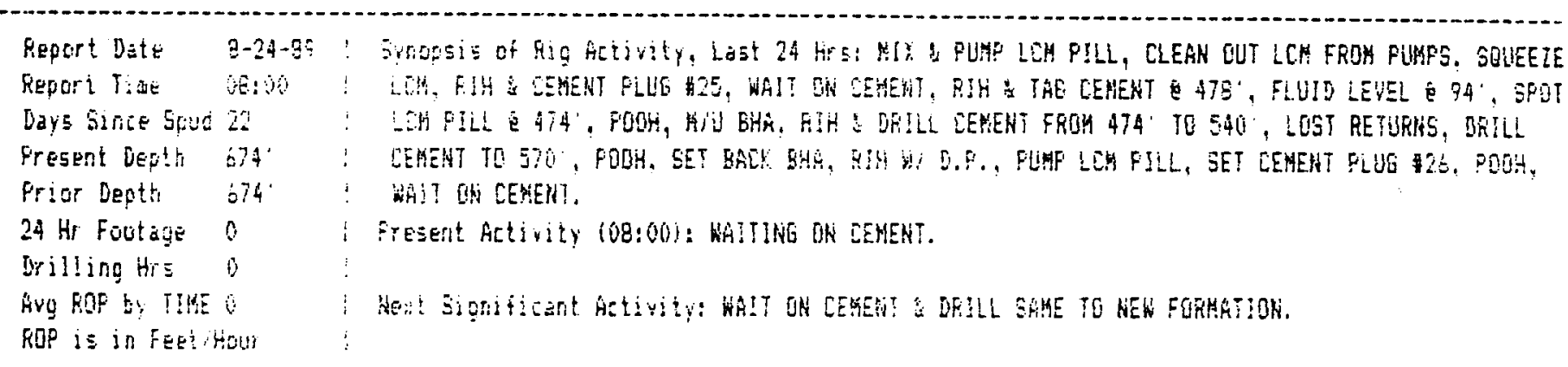

(15

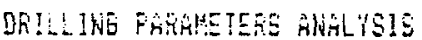

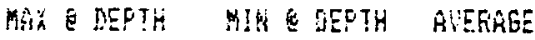

ROP
HOB
RPH
TRQ
PF

\section{LITHOLOG}

Berers! Description: [SeE DIEITÁL HUS LOG for details! b) RAFE I

$k-1 t s$

anps

PSI

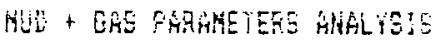

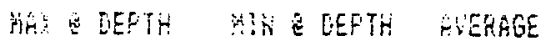

con

cond $1 \mathrm{H}$

CDHO OUT

His

HTDC GAS

abi in

Hat

pH ilis

PH OUT

JEMF IN

TEHP OUIT

tpu

poin kia?!

Deft: HaC?

dyit

units :

$3 b 0$

lat

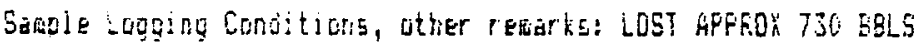

DPSULIWIE FLUIL WHILE DFILLIHG CEMENT E SAD'.
No HEX LITHOLOSY..

(1)

Conn bas i irle bas : Metfarne : Ho Hydrocarbons

CAREIDE LAG Wir: E UNits, SFH =

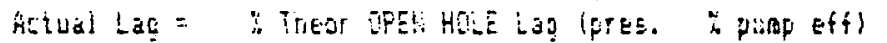

Hud UIS out $=$, 500 grafes Eartide, hinutes Duration

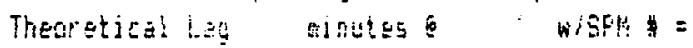

$51 T$ CECOE:

\begin{tabular}{|c|c|c|c|c|c|}
\hline & & & CuH & {$[! j$} & \\
\hline Hio. SIIE & WHE THE & 18 & FTE & HFE & Cokn! I:Th \\
\hline KFE $20^{4}$ & ST & 3 & $354^{\circ}$ & 27.5 & INE \\
\hline
\end{tabular}

Sabrte Sriaker Streen Size: $\# 1=40 / 60$ \& $\$ 2=40 / 60 \mathrm{RESH}$

Latest Seregn Size Ehronge on: 8-?-89 (Start of we]!)

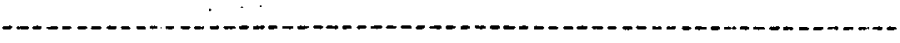

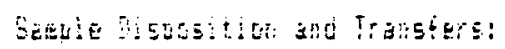

Date:

Irigrval: Fisti to

Sert toikerejued by: 


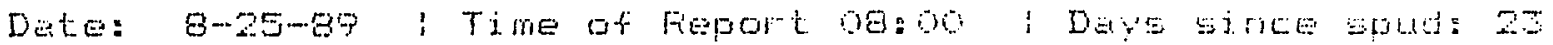

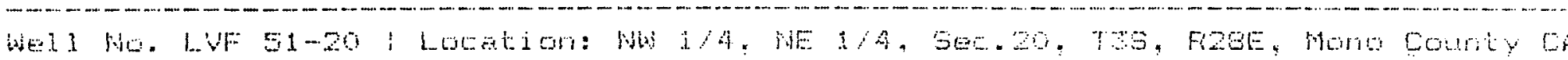

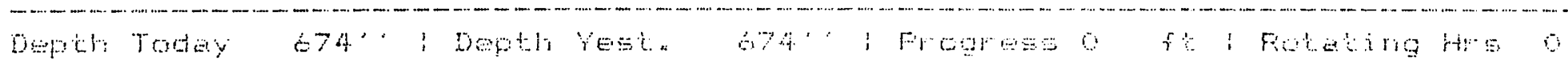

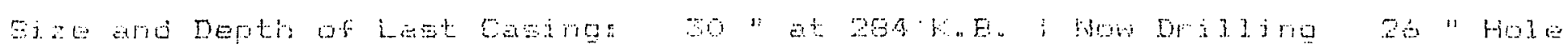
Sit bati:

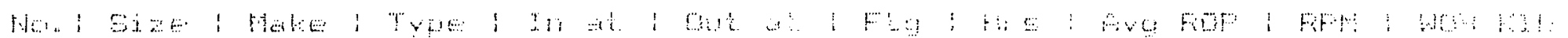

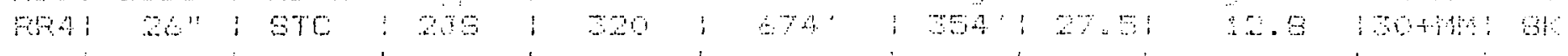

\begin{tabular}{|c|c|c|c|c|}
\hline \multirow{6}{*}{ 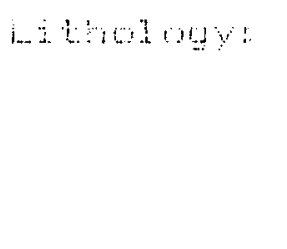 } & $\vdots$ & I1⿴囗十⺝ & $\vdots$ & \multirow{6}{*}{ 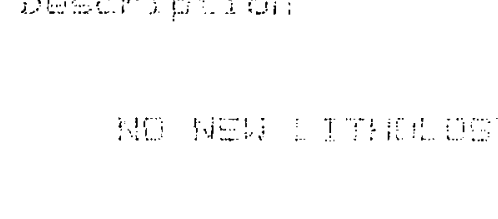 } \\
\hline & $\vdots$ & $\cdots$ & $\mathrm{Ht}$ & \\
\hline & $!$ & $\ldots$ & $5 \mathrm{t}$ & \\
\hline & i & ... & 81 & \\
\hline & : & $\cdots$ & 51 & \\
\hline & $:$ & $\ldots$ & $\because: \quad:$ & \\
\hline
\end{tabular}

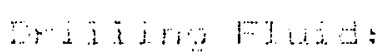

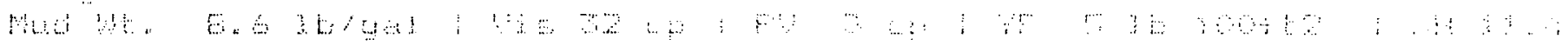

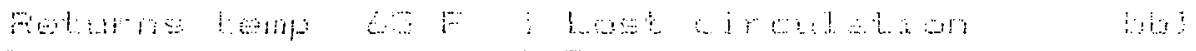

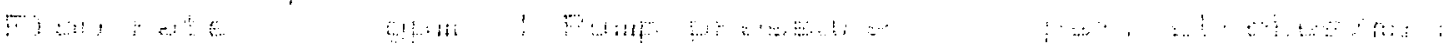

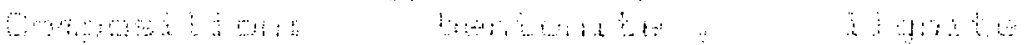

in: :

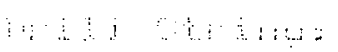

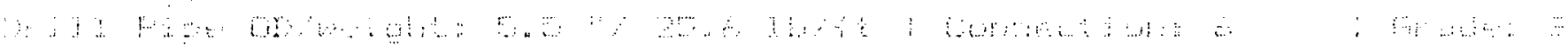

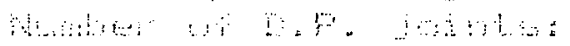

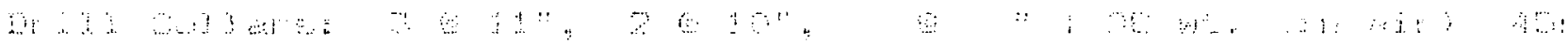

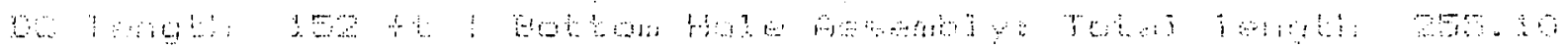

In mor

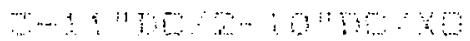

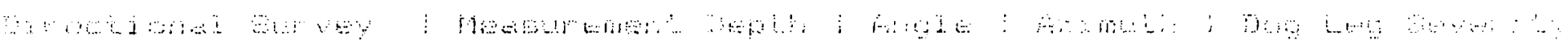

$\begin{array}{ccc}\vdots & \vdots & \vdots \\ \vdots & \vdots & \vdots \\ \vdots & \vdots & \vdots\end{array}$

\section{mon}

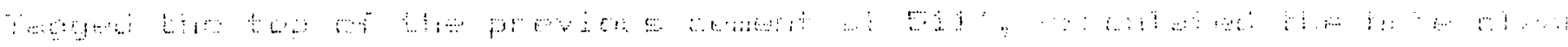

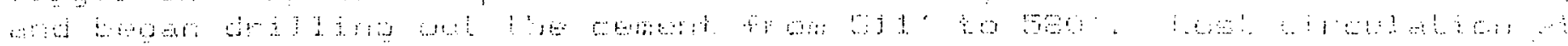

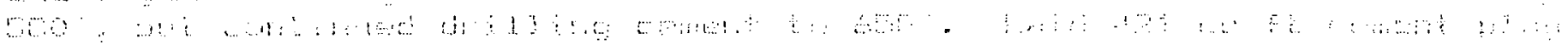

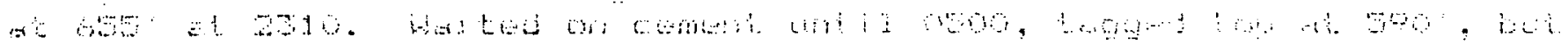

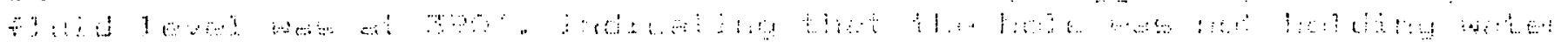

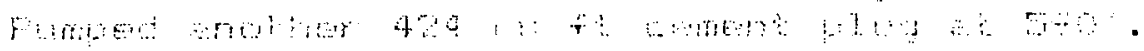

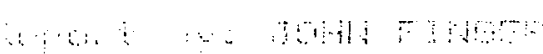


EPOCH HELL LOEGING

\begin{tabular}{|c|c|c|c|}
\hline Report Date & $8-25-99$ & $\mathbf{i}$ & 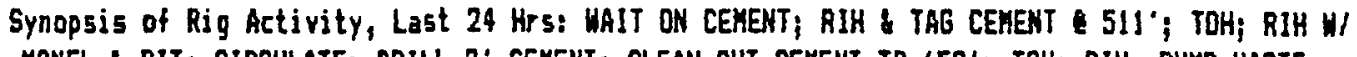 \\
\hline Report Tine & $08: 00$ & $\mathbf{i}$ & MOHEL \& BIT; CIRCULATE; DRILL $2{ }^{\circ}$ CEMENT; CLEAN OUT CEMEHT TO $658^{\prime} ;$ TOH; RIH; PUMP UASTE \\
\hline Days Since Spud & 23 & i & HATER, FRESH HATER, \& CEPALITE; SET CEHENT PLUG 127 ; HAIT ON CEMENT; RIH \& TAG CEKEHT \& \\
\hline Present Depth & $674^{\circ}$ & 1 & $590^{\circ}$; TOH; RIH; PUMP HATER \& HOHCO PILL; SET CERENT PLUG $\$ 28$ AT $590^{\prime}$. \\
\hline Prior Depth & $674^{\circ}$ & : & \\
\hline $24 \mathrm{Hr}$ Footage & 0 & 1 & Present Activity (08:00): SET CEHEKT PLUG 28 \\
\hline Drilling Hrs & 0 & : & \\
\hline $\begin{array}{l}\text { AYg ROP by TIHE } \\
\text { ROP is in Feet/ }\end{array}$ & & i & Next Significant Activity: MAIT ON CEMENT \& DRILL SAME TO MEH FORMATIOH. \\
\hline
\end{tabular}

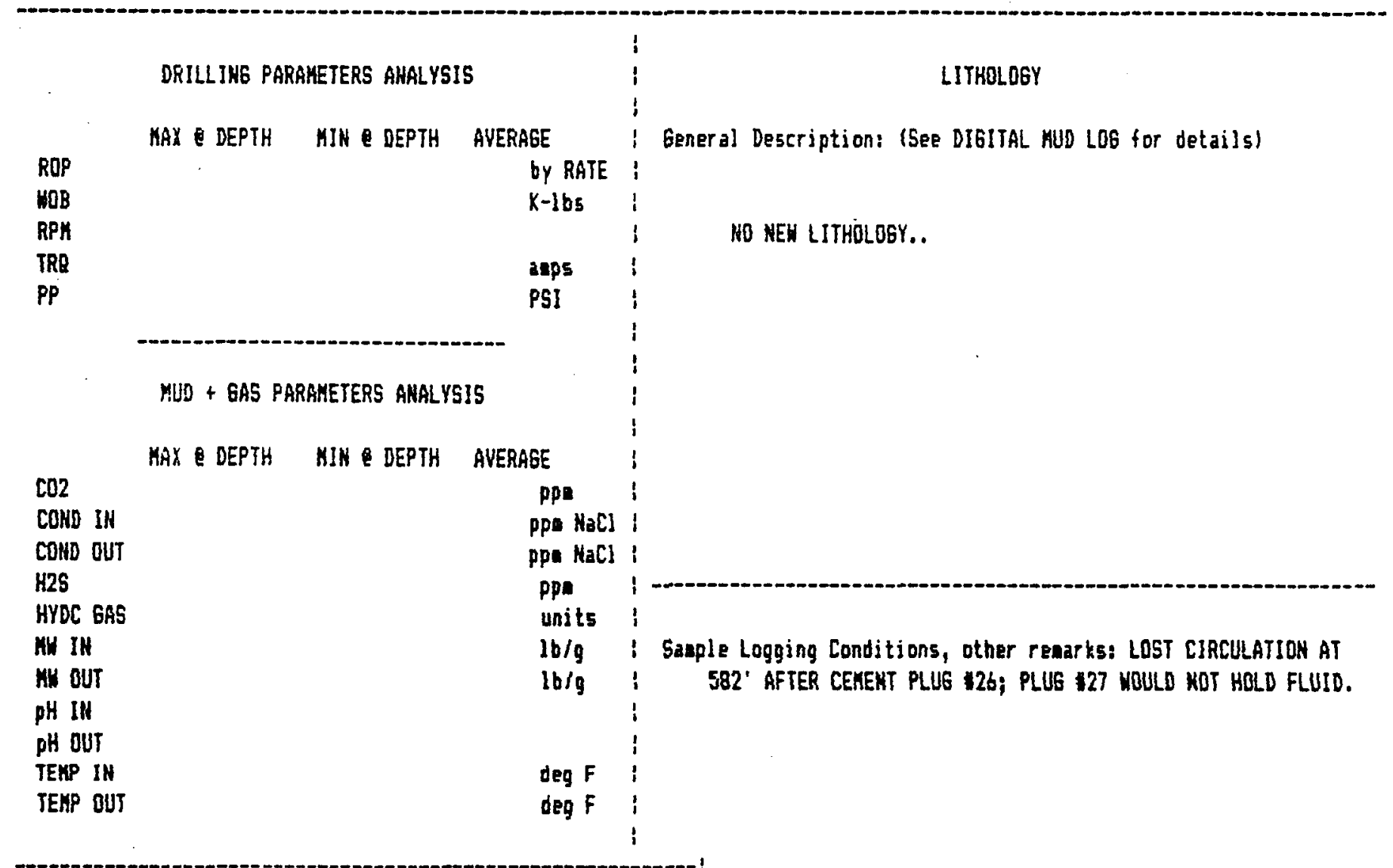

Conn Ga5 / Trip Gas / Methane : No Hydrocarbons

CARBIDE LAG ain ', units, SPH =

Actual Lag $=2$ Theor OPEN HOLE Lag Ipres. I puop eff\}: Hud VIS out $=$, 500 grans Carbide, Minutes Duration I Theoretical Lag, winutes $1 \mathrm{~W} / \mathrm{SPH}=$

BIT RECORD

CUM CUA

N8. SIIE KAKE/TYFE IN E FTG HRS CONDITION

RA4 26" STC 235 320 $354^{\circ} 27.5$ IHC $-1$
Saeple Shaker Screen Size: $\$ 1=40 / 60$ \& $\$ 2=40 / 60$ MESH

Latest Screen Size Change on 8-2-89 (Start of well) 


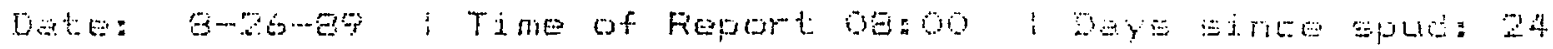

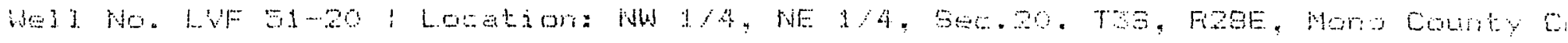

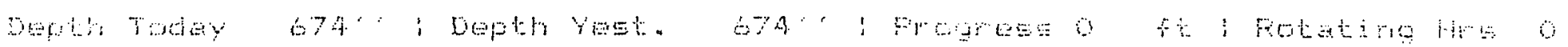

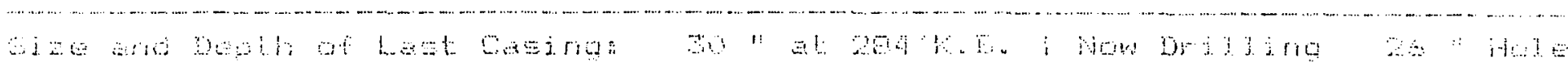
Yis

Ho : कम का

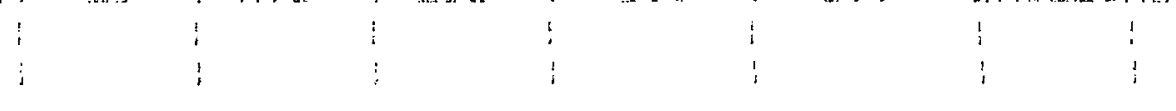

\begin{tabular}{|c|c|c|c|}
\hline i. & $\vdots$ & 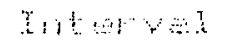 & \\
\hline & $!$ & & $\because t$ \\
\hline & $\vdots$ & $\cdots$ & $F$ \\
\hline & $\mathfrak{i}$ & $\ldots$. & $\therefore$ \\
\hline & $\vdots$ & $\cdots$ & $F$ \\
\hline & $\vdots$ & $\cdots$ & 6 \\
\hline
\end{tabular}

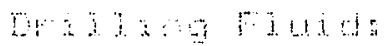

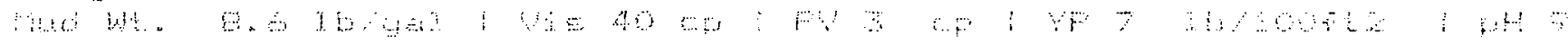

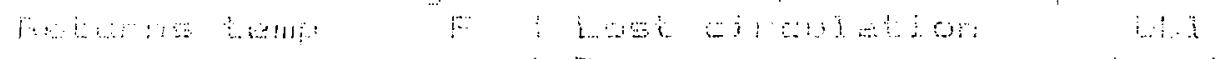

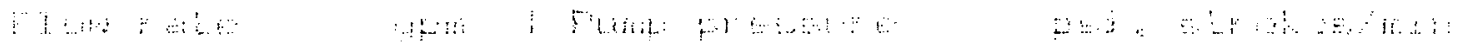

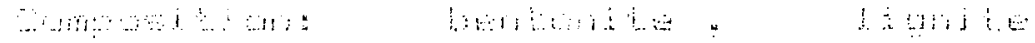

: : : :

U⿻

y

Hen

अ

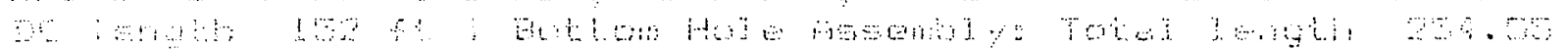

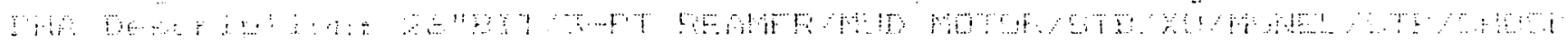

3 i 1 :

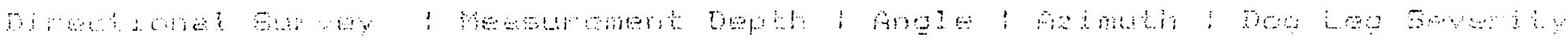

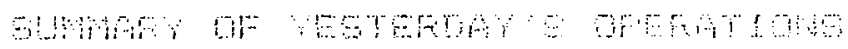

a.

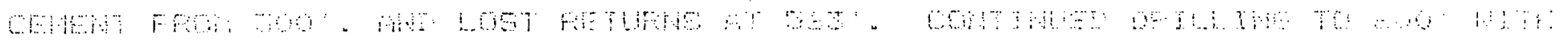

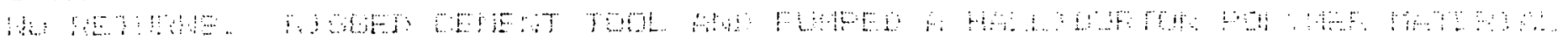

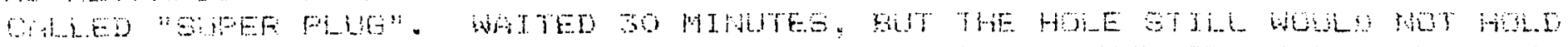
ra अ

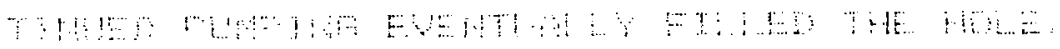

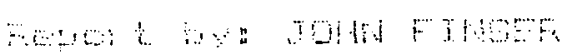


EPOEYH WELLL LOOGGINLF

\section{DOE/8ANDIA MAGMA ENERGY WELL LVF 51-20 DAILY MUD LDG REPDRT}

\begin{tabular}{|c|c|c|c|}
\hline Report Date & $8-26-89$ & 1 & Synopsis of Rig Activity, Last 24 Hrs: haIT OH CEMENT, RIH \& TAG CEMENT $500^{\circ}$, CIRE \& \\
\hline Report Tine & $08: 00$ & i & COHDITION HUD, RIH WITH BHA, DRILL CEMENT TO 582', LOSSING CIRCULATION E 563', PUMP LCH PILL \\
\hline Days Since Spud & 24 & $i$ & DAILL CEHEKT TO $600^{\circ}$, POOH, KIH \& SET FOLYHER PLUE, WAIT OH FOLYHEK, PUMP 350 BELS FLUIO INTO \\
\hline Present Depth & $674^{\prime}$ & $i$ & 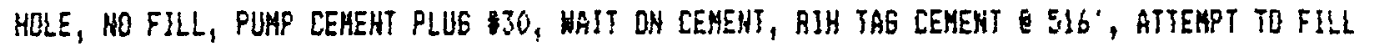 \\
\hline Prior Depth & $674^{\circ}$ & : & HOLE, POOH. \\
\hline $24 \mathrm{Hr}$ Footage & 0 & i & Present Activity 108:001: PREPAR3HG TO RIH CEMENT \\
\hline Drilling Hrs & 0 & $\mathbf{i}$ & \\
\hline $\begin{array}{l}\text { AYg ROP by TIME } \\
\text { ROP is in Feet/l }\end{array}$ & $\begin{array}{l}0 \\
\text { Hour }\end{array}$ & $\vdots$ & Hext Significant Activity: CEMENT FLUG $\$ 31$ \\
\hline
\end{tabular}

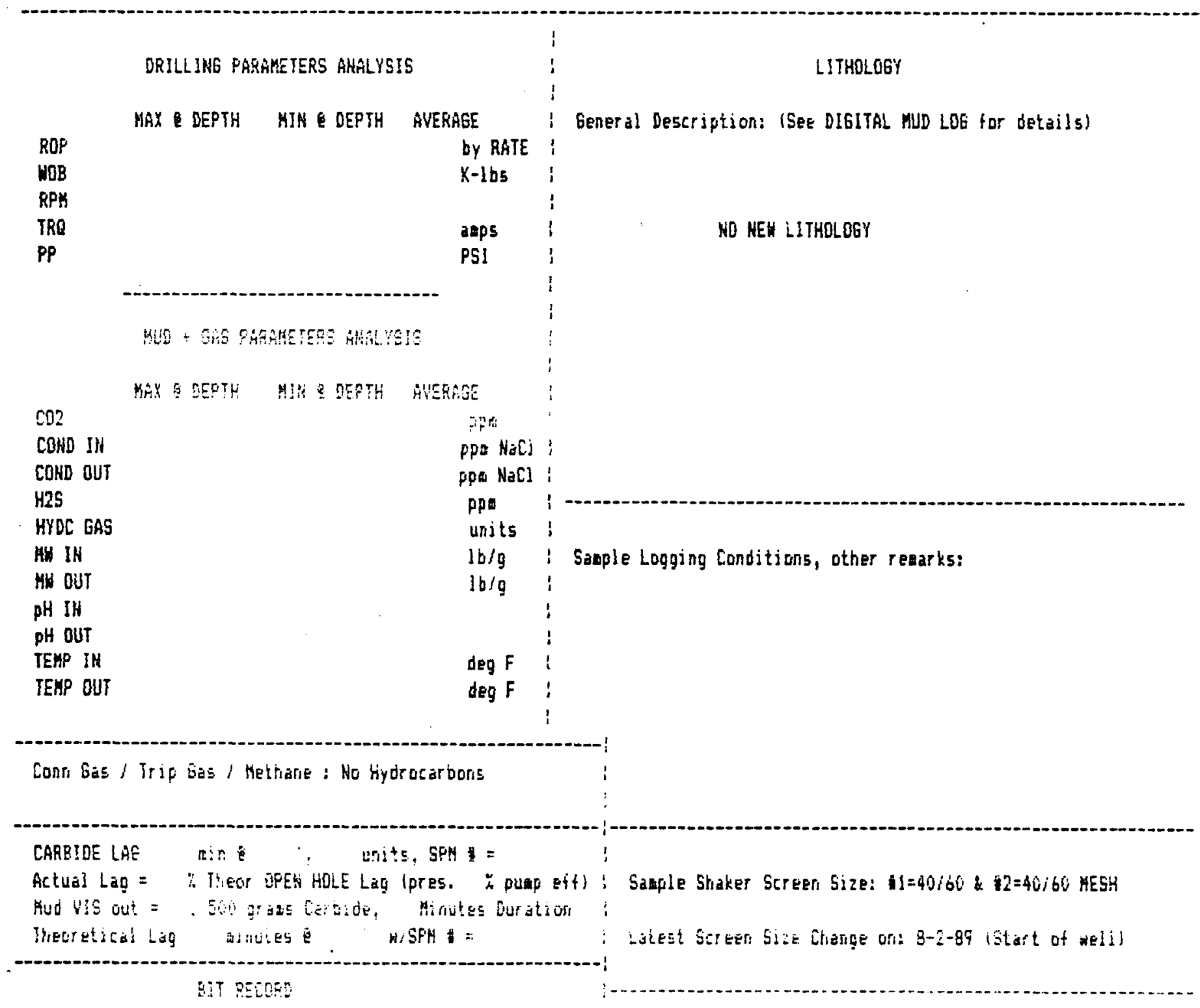

Rep

MANE/THE IN F FTO HAS EONDITION

RR4 26. STC 2JS 500 $100^{\circ} /$ DGILLING CEMENT

Sample Disposition and Traristers:

Date:

Interval: from to

Sent to/keceived by: 


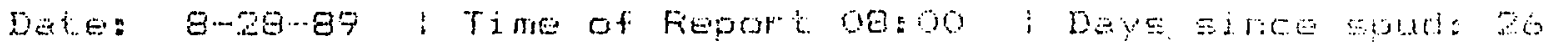

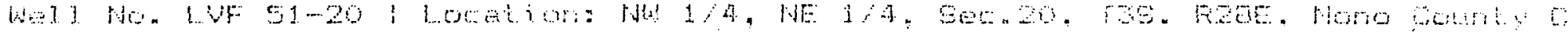

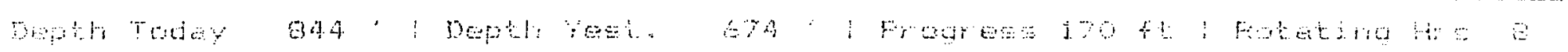

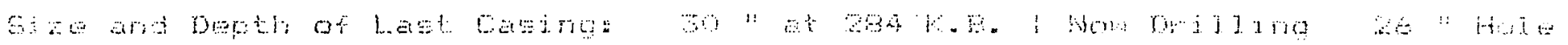
(1): 1: : : : :

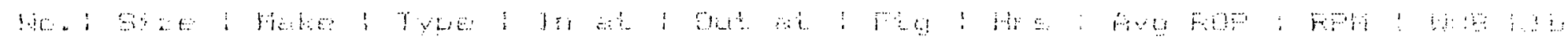
Fै: 26" : $\begin{array}{ccc}i & \vdots \\ \vdots & \vdots\end{array}$

:.

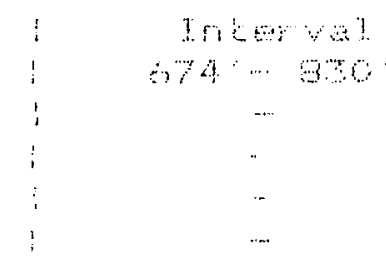

$\begin{array}{cc} & \vdots \\ \pi & \vdots \\ \ddots \vdots & \vdots \\ \vdots & \vdots \\ \ddots & \vdots \\ \vdots & \vdots\end{array}$

\begin{abstract}
$\mathrm{Ten}=\mathrm{BH}$

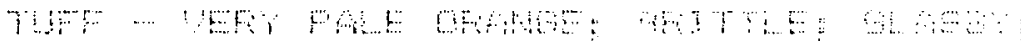

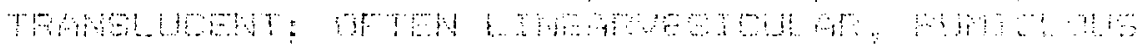

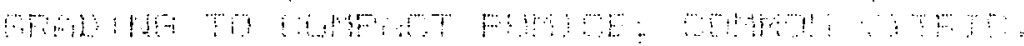

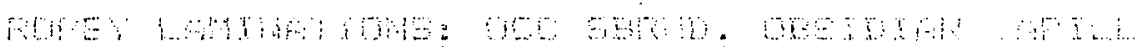

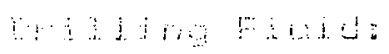

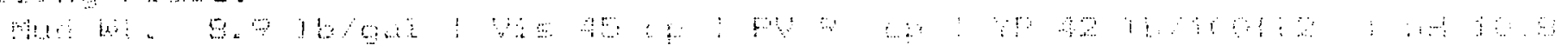

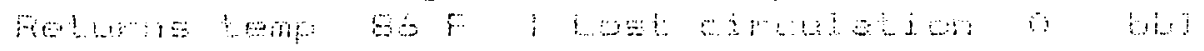

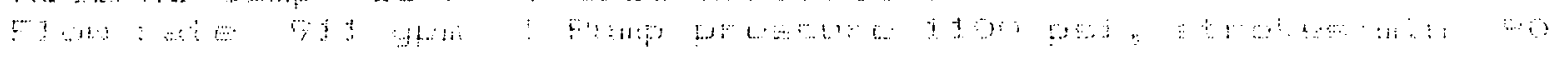

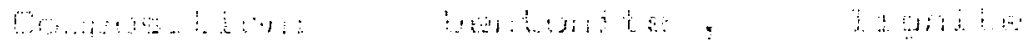

a.:

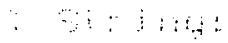

$\therefore: 1: 00 \%$ :

namer $\therefore$ :

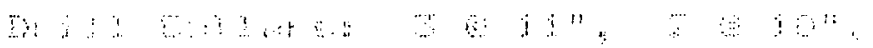

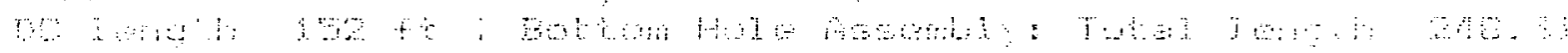

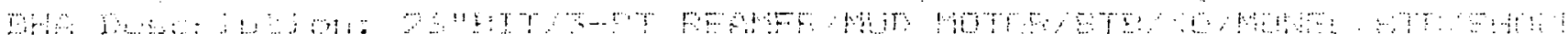
- i i : $: \cdots$

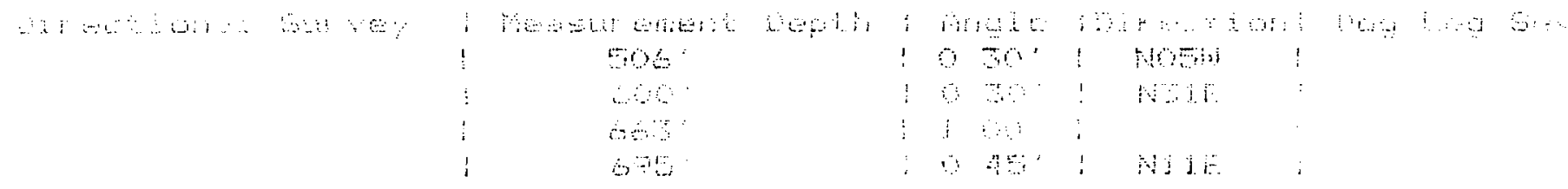

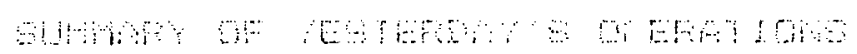

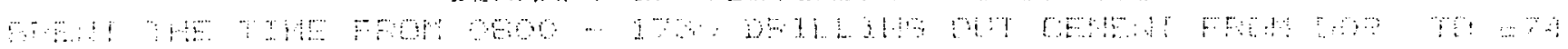

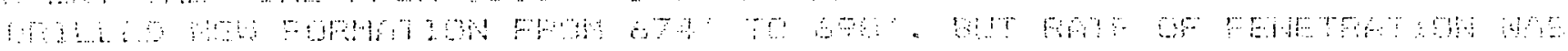

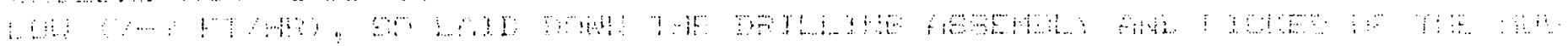
19т,

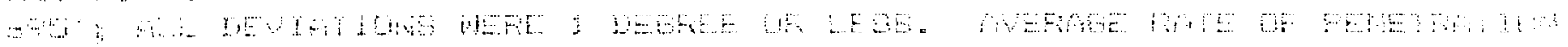
19

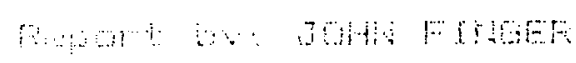


EFOCH UELL 1 WGSIHS

\section{DOE/SANDIA MAGMA ENEREY WELL LUF 51-20 DAILY MUD LDG REFOFT}

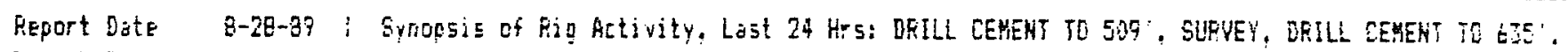

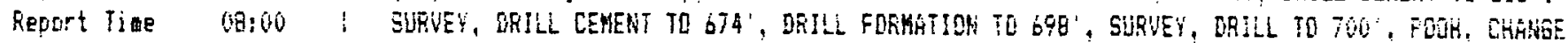

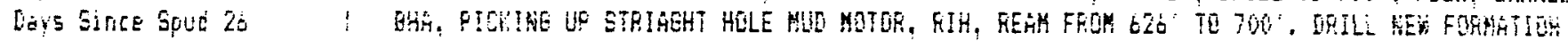

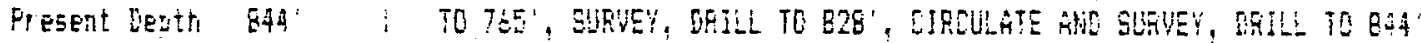

Prior Desth $674^{\prime}$

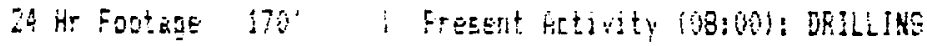

Drilling ths a

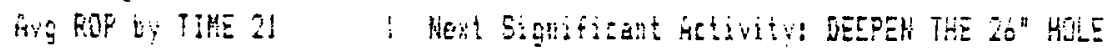

Fot is ir Fegitifour

\section{DRILIHE FARBHETES RHALES}

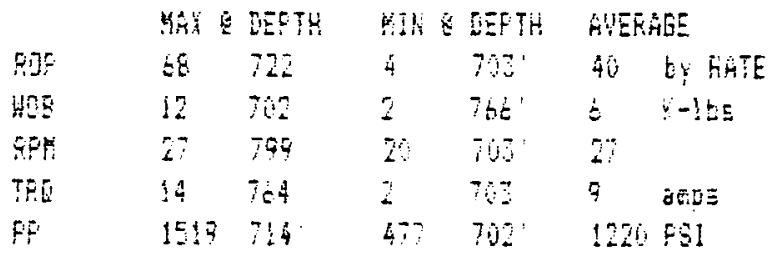

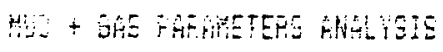

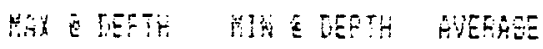

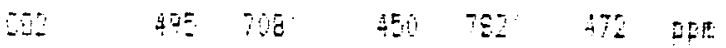

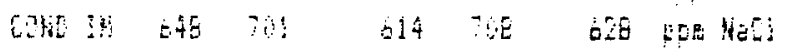

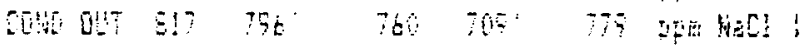

Hes 0 b bitit

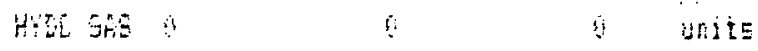

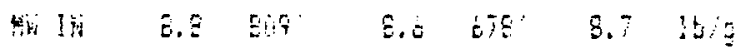

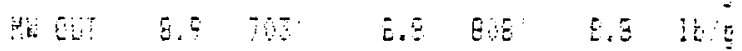

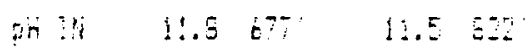

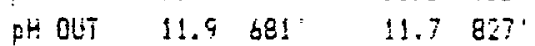

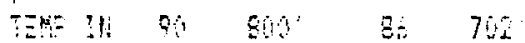

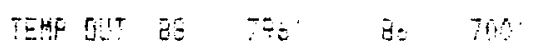

\section{LTHULG:}

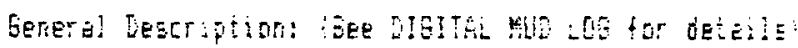

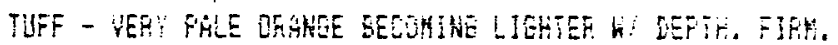

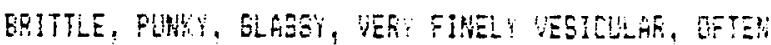

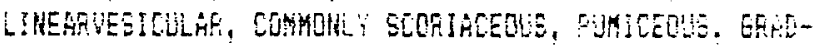

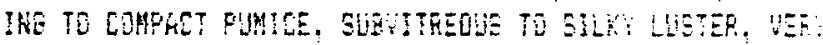

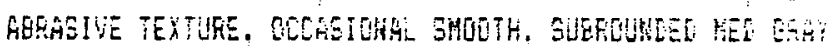

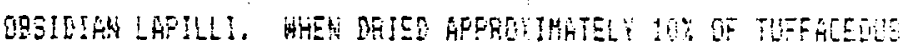

Fock HILL F!on?.

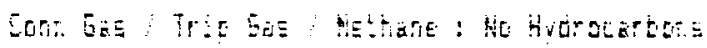

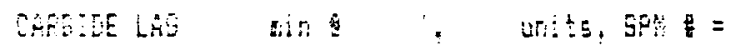

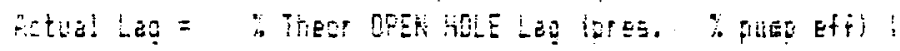

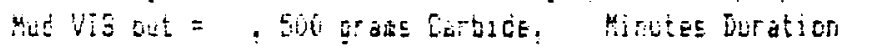

Theoretica! La?

ainutese, wSPM \# =

S:T RECUF

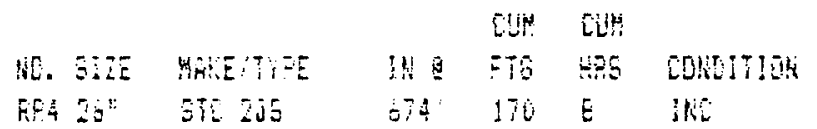

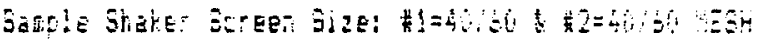

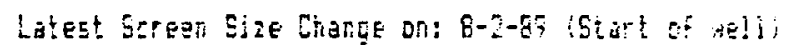

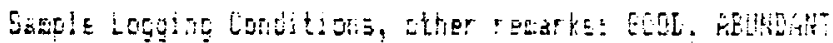

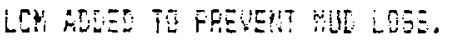




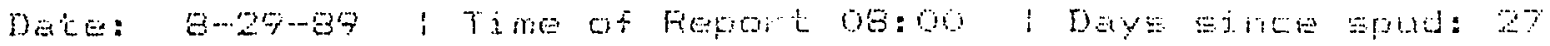

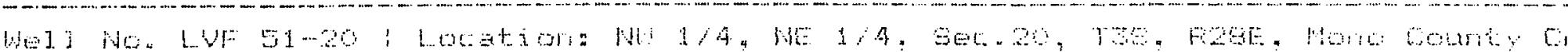

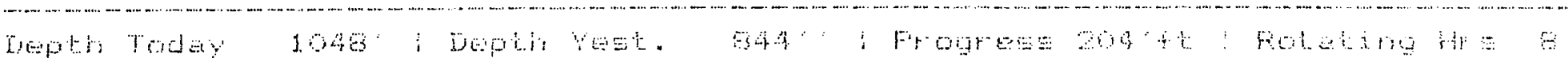

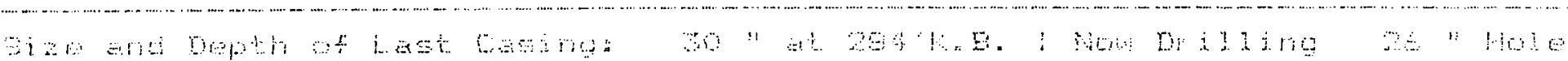

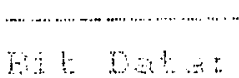

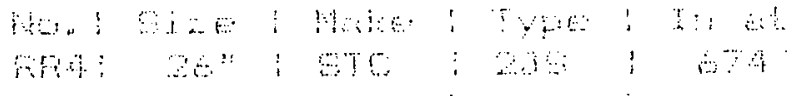

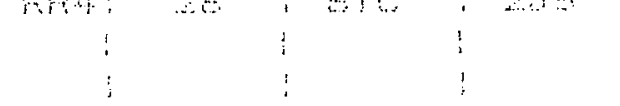

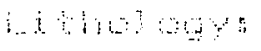

In: $1,4 y$ अ क क an $\mathrm{i}$
W

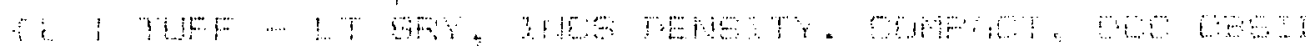
;

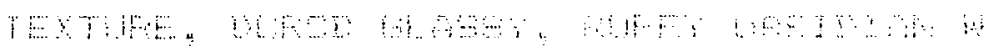
(n)
9. ind in

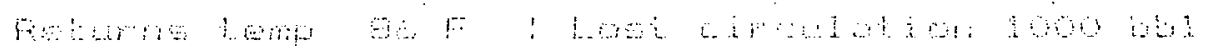

‥

-

: : : : : :

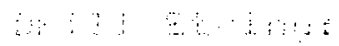

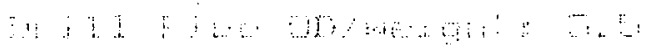

A thit

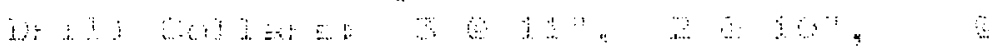

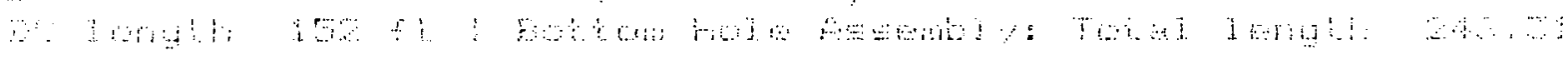

¿

$$
\therefore \text { a }
$$

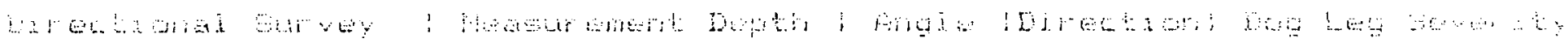
$\because \ldots$

a

:

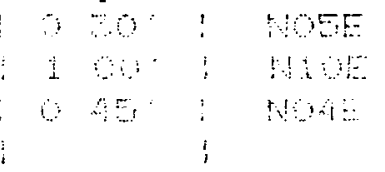

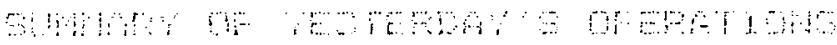

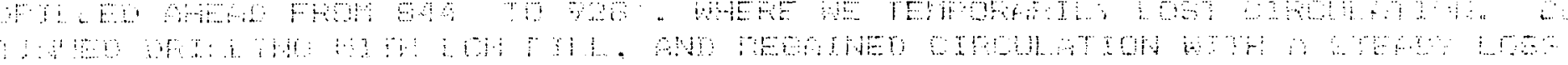

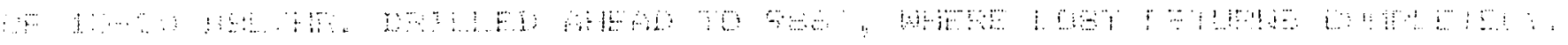

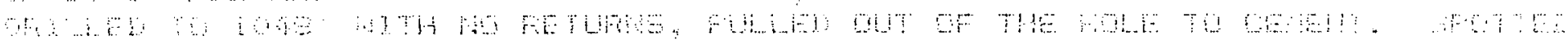

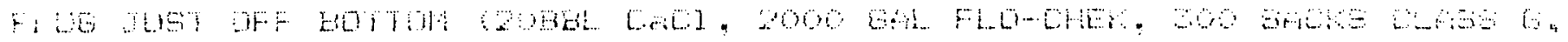

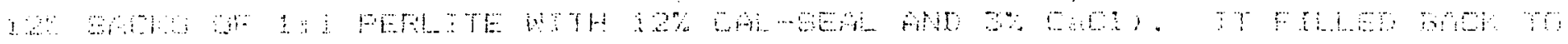

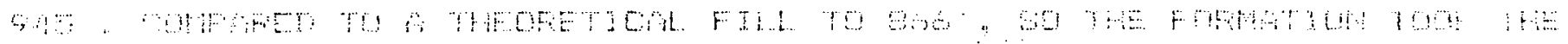

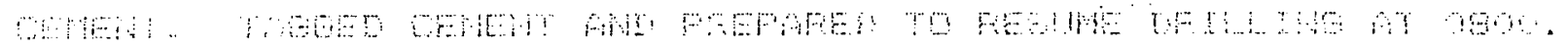

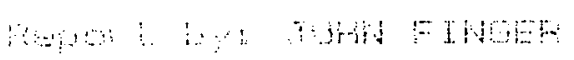


EOSH JEL $\quad$ OOELUG

DOE/SANDIA MAGMA ENERGY WELL LVF 51-20 DAILY MUD LOG REPORT

\begin{tabular}{|c|c|c|c|}
\hline Recort Date & $6-25-85$ & 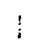 & 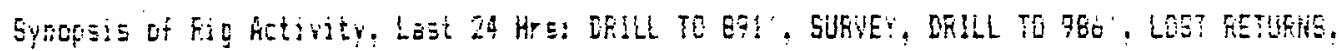 \\
\hline Revert itize & $08: 60$ & 1 & 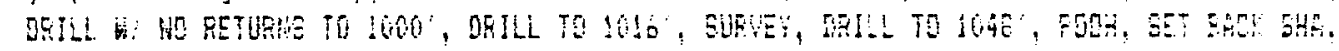 \\
\hline bays Eince jọun & 27 & 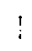 & 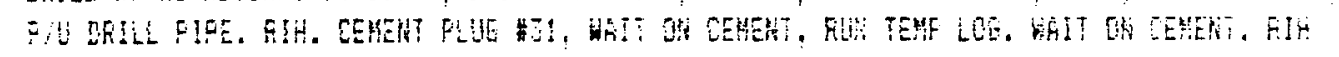 \\
\hline PREEert LEEti & $1948^{\circ}$ & ? & THE CEAEWT E TAE CIRE, IET LCH FILL, POQK. \\
\hline Priar Depth & 84 & ! & \\
\hline $24 \mathrm{Hr}$ Ectidag & 264 & ! & 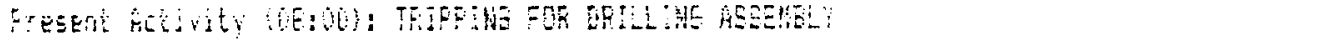 \\
\hline 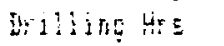 & $\bar{E}$ & ! & \\
\hline 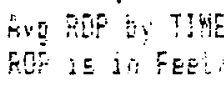 & $\begin{array}{l}75 \\
\text { Hotj }\end{array}$ & & 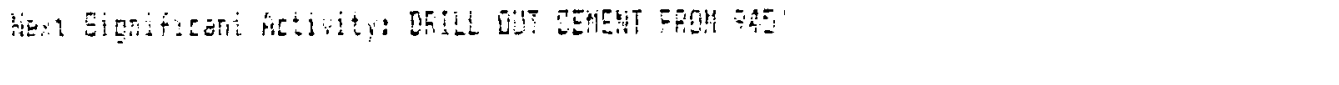 \\
\hline
\end{tabular}

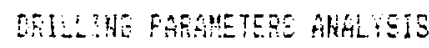

\begin{tabular}{|c|c|c|c|c|c|c|}
\hline & $4 \%:$ & DEFTH & 告证 & E METH & Pinses & \\
\hline FE: & 9 & $1022^{\circ}$ & $\bar{E}$ & $4: ?$ & je & DY PATE \\
\hline We & 12 & Es: & $=$ & $9 ! 3$ & 5 & $1-1 t=$ \\
\hline EF & $2 E$ & PEE & 28 & $8 \div$ & 26 & \\
\hline TEL & 4 & 435 & 8 & 507 & 15 & ange \\
\hline of & 1.58 & 32 & Q5: & 50 & 126 & $5:$ \\
\hline
\end{tabular}

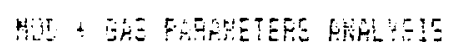

\begin{tabular}{|c|c|c|c|c|c|c|}
\hline & ME: & EFT & 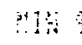 & DETH & AVET & 65 \\
\hline 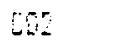 & 511 & 7 & it & $1030^{\circ}$ & 421 & Fon \\
\hline Eond IH & 87 & 95 & 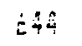 & $20:$ & $6 ? 7$ & ping Nali \\
\hline Coni su! & 79 & Eack & 0 & $16+\bar{e}$ & 085 & pom $\mathrm{HaC}$ \\
\hline$\therefore 28$ & $a$ & & 0 & & $\theta$ & 5 \\
\hline HOS GAS & 0 & & $i$ & & $\theta$ & witits \\
\hline H $1 \%$ & $B .7$ & 72 & 5.7 & $\operatorname{lng}$ & $E . E$ & $\ln 9$ \\
\hline $4: 0 ! \bar{T}$ & 5.5 & 905 & B. & $\because 80^{\prime}$ & 5.5 & 250 \\
\hline 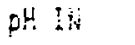 & $\$ 1.8$ & 34 & 125 & 100 & 11.7 & \\
\hline oH SUT & $\because 1.5$ & 370 & 11.7 & $1022^{\circ}$ & II.E & \\
\hline EHE in & $=5$ & $=40^{\circ}$ & $5:$ & 108 & $9:$ & $\operatorname{tg} F$ \\
\hline TE增 & 98 & 95 & $\vdots$ & 1046 & B: & oge 5 \\
\hline
\end{tabular}

\section{L.THL:}

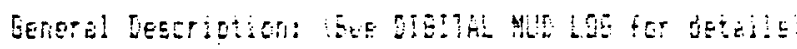

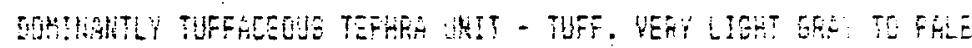

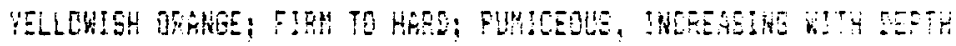

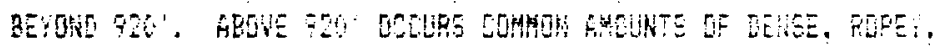

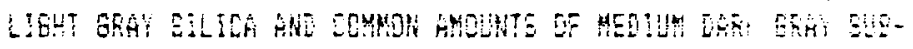

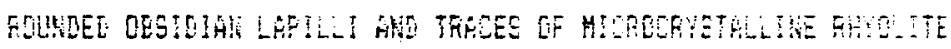

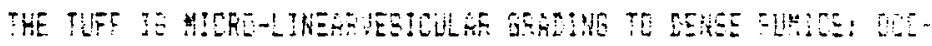

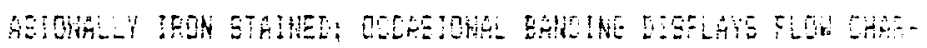
STEFISTISE.

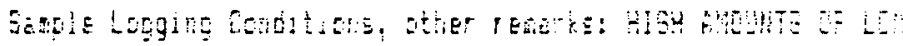

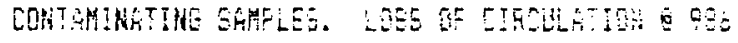

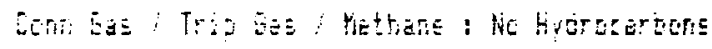

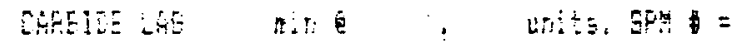

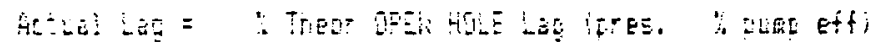
Mud vis out $=$, sou quars Carbice. Hinutes furation

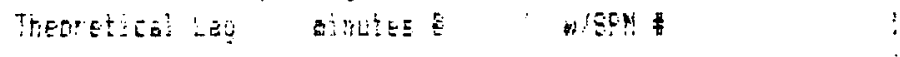
E!T

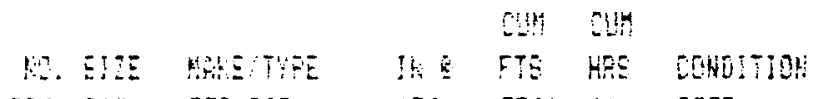

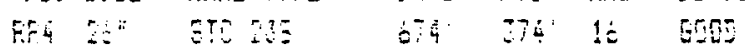

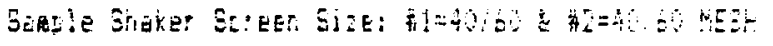

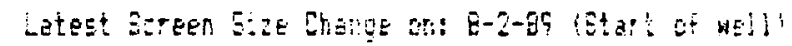




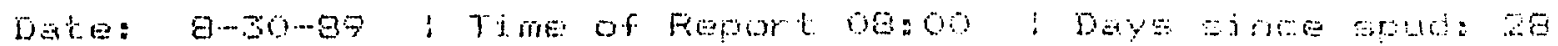

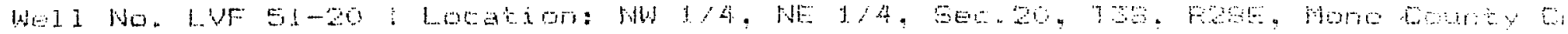

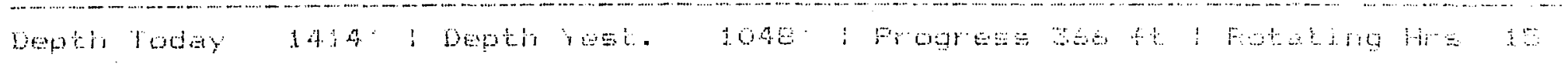

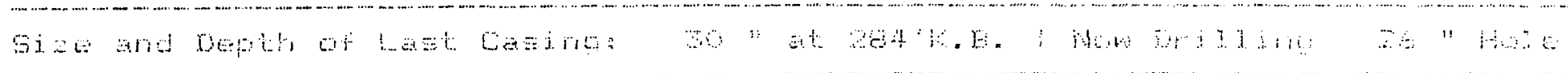

atista:

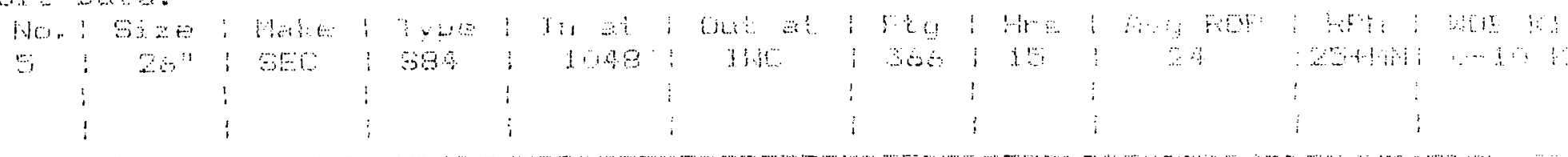

thentosy

\begin{tabular}{|c|c|c|c|}
\hline$\vdots$ & $A: 60$ & $+a$ & \\
\hline$!$ & $\operatorname{san}{ }^{\circ}$ & 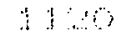 & $\because t$ \\
\hline ; & if 120 & sकी & $4 \div$ \\
\hline$\vdots$ & $\ldots . .$. & & 1 \\
\hline$!$ & $\cdots$ & & $F$ \\
\hline$\vdots$ & $\cdots$ & & $\because$ \\
\hline
\end{tabular}

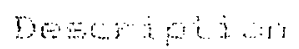

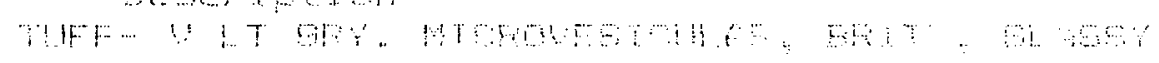

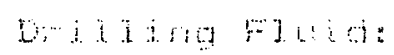

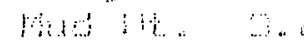

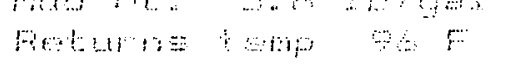
F⿻

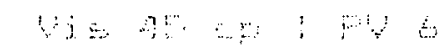

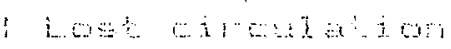
$+1$
Cim
a
$\begin{array}{llll}1 & 4 & 4 & 4 \\ 1 & 1 & 0\end{array}$

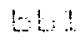
[1: $:: ;$

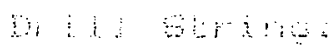

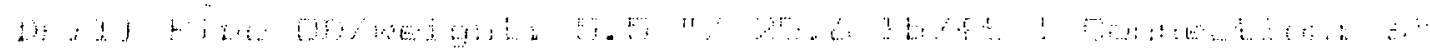

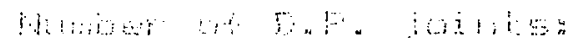

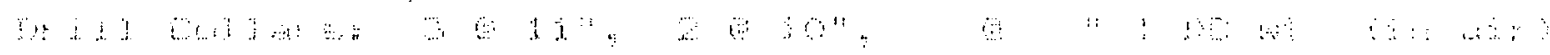

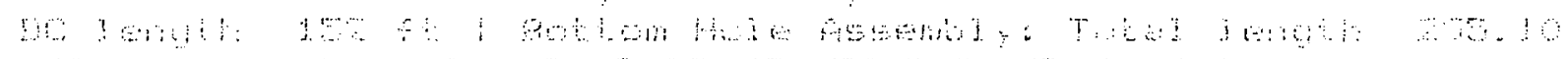

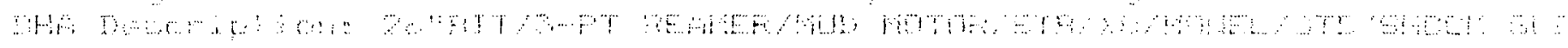

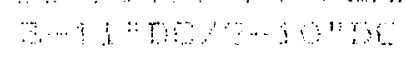

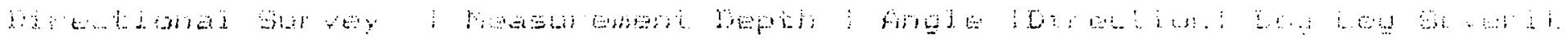

\begin{tabular}{|c|c|c|c|c|c|}
\hline 9 & $\vdots$ & 1 & 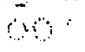 & $\vdots$ & 3 \\
\hline 40 & $!$ & ) & $\because \vdots$ & $\vdots$ & id \\
\hline $4: 3$ & 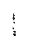 & $!$ & a & $\vdots$ & $\therefore$ \\
\hline 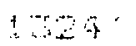 & $i$ & 1 & $i$ & $i$ & $\because$ \\
\hline
\end{tabular}

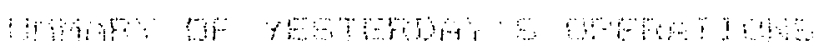

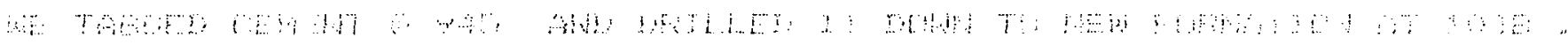

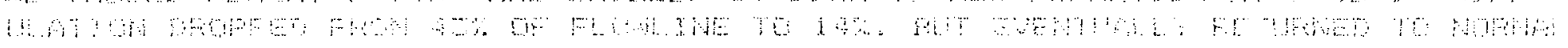

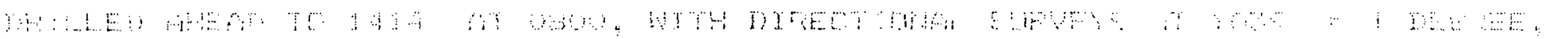

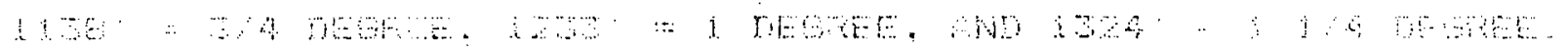

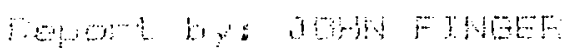


EPOCH WEL: IOSEINE

DOE/SANDIA MAGMA ENEREY WELL LUF 51-20 DAILY MUD LOG REPDFT

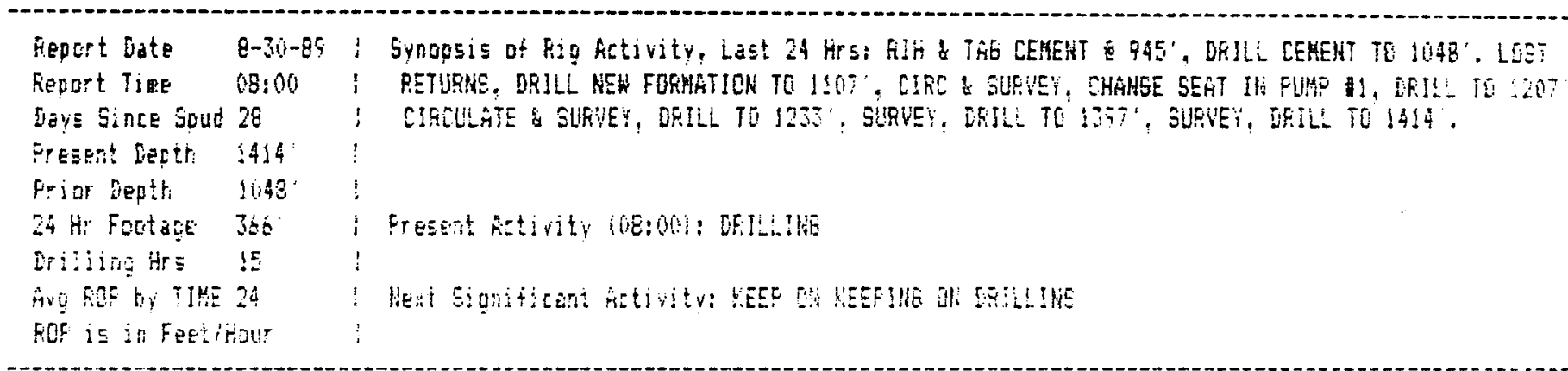

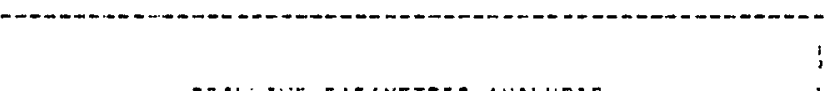

\section{DRILIME FIFAETERS AMALYS:S}

\begin{tabular}{|c|c|c|c|c|c|c|}
\hline & Shi: & DETH & \multicolumn{2}{|c|}{ 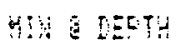 } & \multicolumn{2}{|c|}{ AUEFAEE } \\
\hline R.jp & 100 & 1085 & 0 & 127 & 3 & QV RHE \\
\hline 135 & 32 & 215 & $\therefore \bar{B}$ & 229 & 3 & $1-1 b=$ \\
\hline EFt: & $2 E$ & 190 & 25 & $12: 5$ & 25 & \\
\hline The & 35 & $\operatorname{sige}$ & $?$ & 137 & 8 & 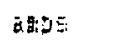 \\
\hline EF & 1717 & 938 & 70 & $2 t ?$ & $15 E$ & 58 \\
\hline
\end{tabular}

\begin{tabular}{|c|c|c|c|c|c|c|}
\hline \multirow[b]{3}{*}{12} & \multicolumn{6}{|c|}{ 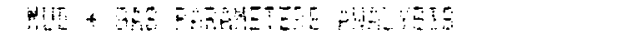 } \\
\hline & this & 805 & $\because \ldots$ & SET: & AIE: & 68 \\
\hline & 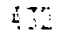 & 10 & 24 & $\because 7$ & 35 & Finti. \\
\hline CH:TI: IH & 30 & 16 & 53 & 1,0 & 2 & pa: 165 \\
\hline औf & 2872 & :CE & $5-$ & 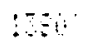 & 10 & Det $\operatorname{lin}$ \\
\hline 42 & 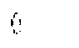 & & b & & $\therefore$ & $\operatorname{pen}$ \\
\hline Hits 95: & i & & $\because$ & & $\therefore$ & Uat: \\
\hline 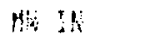 & E.E & $2 \cdots+1$ & $\therefore .7$ & $10=$ & 3.5 & $\operatorname{tog}$ \\
\hline 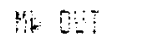 & $E 2$ & $:-5$ & 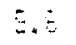 & ISE & $E .5$ & 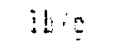 \\
\hline 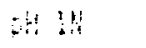 & $\because \therefore$ & 15 & $\therefore \therefore$ & 95 & 16 & \\
\hline pt but & $2: 5$ & 1974 & $\therefore .0$ & $596^{\circ}$ & 21.5 & \\
\hline SEtE It & 75 & $1380^{\circ}$ & 3 & 10.9 & 87 & $25=$ \\
\hline Tye & 9 & 1:9! & $E$ & $: 19$ & 3 & $d E=5$ \\
\hline
\end{tabular}
LiTHrigs

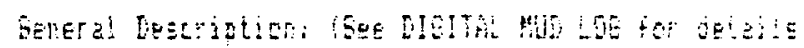

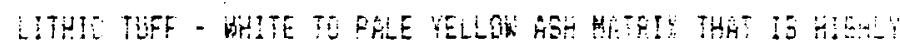

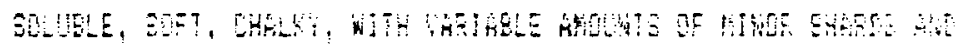

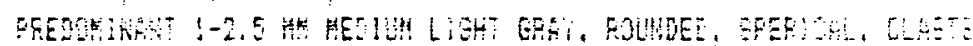

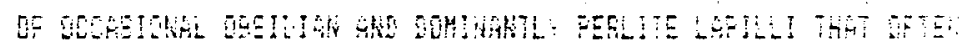

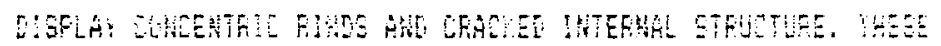

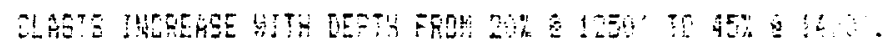

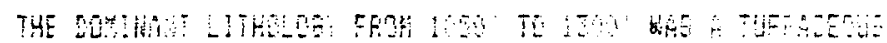

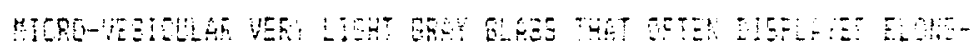

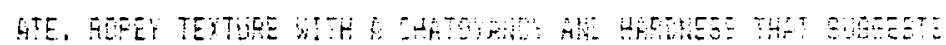

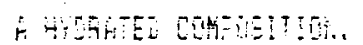

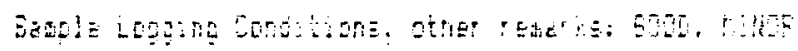

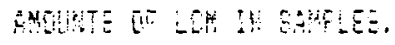

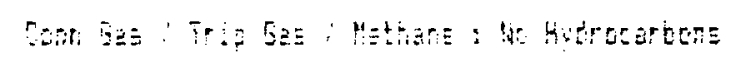

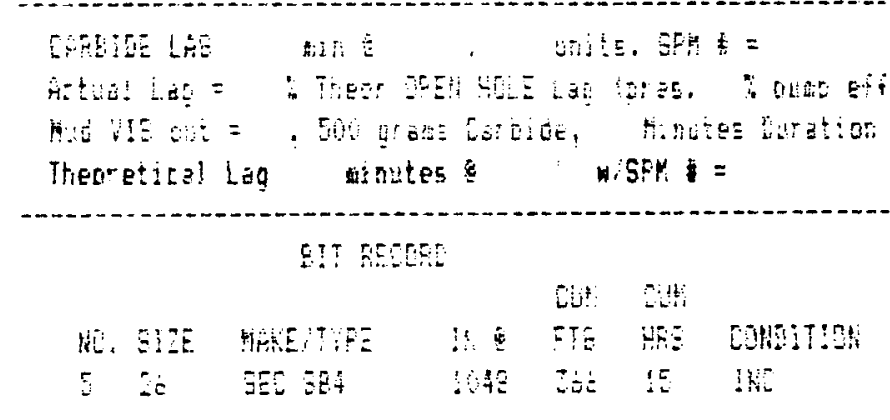

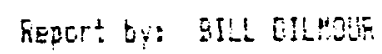

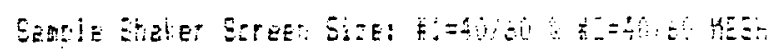

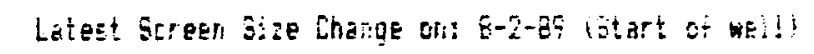

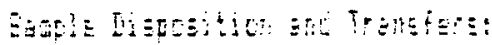

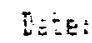

intervis: Fran : is

Ser! torecelad bu? 


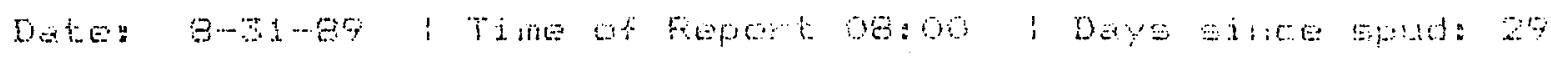

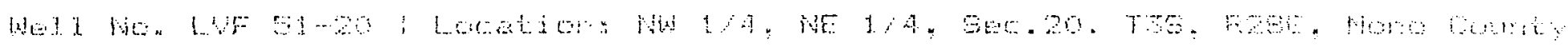

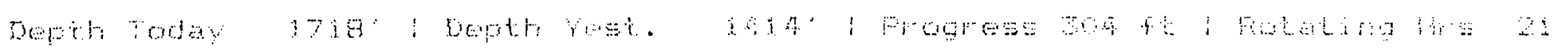

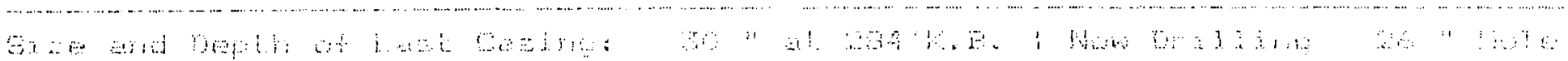

Eis is is is:

Hen :

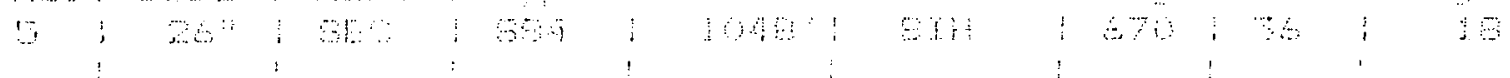

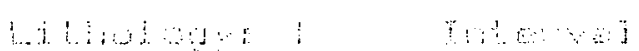

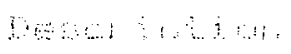

14त् $19 \%$

and and

$130-190$

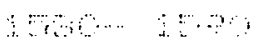

$5 \% \quad 1 \% 0$

6. 30

(4)
mats

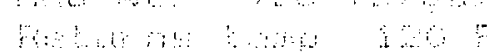

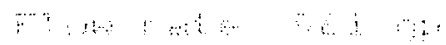

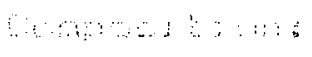
: : : :

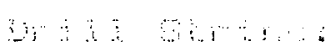

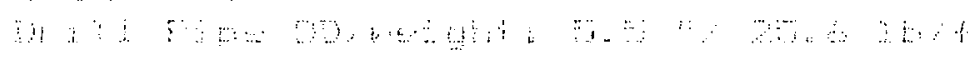

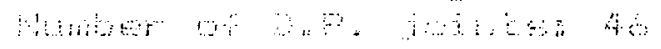

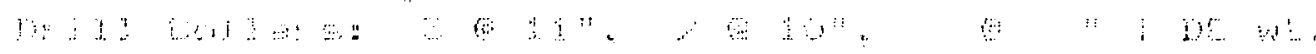

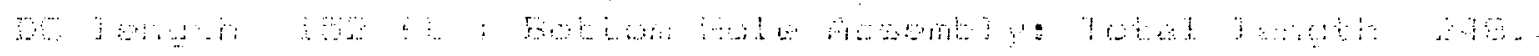

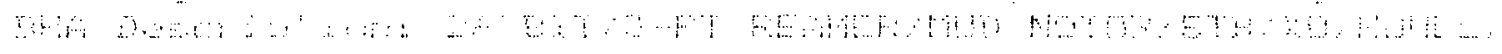

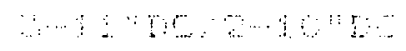

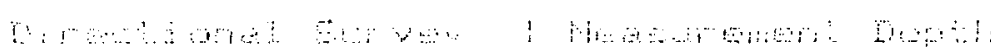

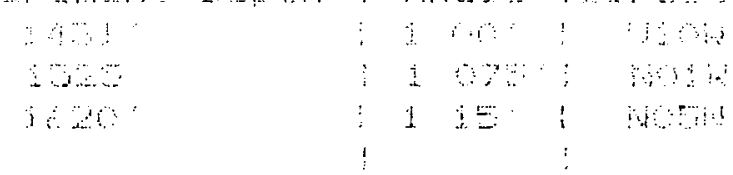

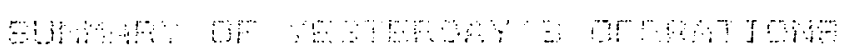

\% - matra

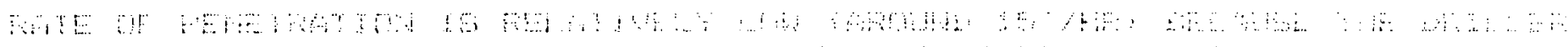

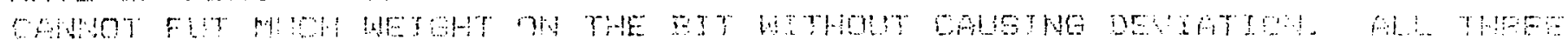
ㄱom ;. .

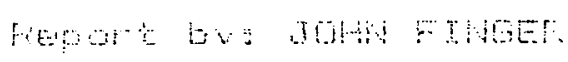


EFOCH WELL LOLGQING

DOE/SANDIA MAGMA ENERGY WELL LUF 51-20 DAILY MUD LOG REPORT

Report Date 8-31-89 ' Synopsis of Rig Activity, Last 24 HYE: DRILL TO 1491', SURVEY, DRILL TO 1573', WORK ON MOTORS Report TiEe Og:00 i ORILL TO 1585', SURVEY, DRILL TO 1690', SURVEY, ORILL TO 17!8'.

Days Since Spud 29

Present Deptr: 1719

Prior Depth 1414

24 Hr Footage 304' : Fresent Activity (08:00): DR!L! IKo

Drilling Hrs 2 !

AVg ROP by TIME :S

ROP is in Feet/hour

Hext Sigrificant Activity: GRlLL..............

ORILLING PATSHEIESS RMELYSIS

\begin{tabular}{|c|c|c|c|c|c|c|}
\hline \multirow[b]{2}{*}{$R P P$} & \multicolumn{2}{|c|}{ HAY GEFTH } & \multicolumn{2}{|c|}{ KIN \& DEPTH } & \multicolumn{2}{|c|}{ A'VERAGE } \\
\hline & 32 & $1591^{\circ}$ & 4 & $1588^{\circ}$ & 17 & by RATE \\
\hline HOB & 9 & $14 \leq 4^{\circ}$ & 2 & $1580^{\circ}$ & $\dot{0}$ & $x-1 b s$ \\
\hline RPn & 30 & $1670^{\circ}$ & 24 & $1461^{\prime}$ & 33 & \\
\hline TRQ & 28 & 1556 & 8 & $1647^{\circ}$ & 9 & asps \\
\hline$P P$ & 2011 & 1608 & 802 & $! 548^{\circ}$ & 1760 & PSI \\
\hline
\end{tabular}

MUUS + GAS PRFGKHETERS ANALYSIS

\begin{tabular}{|c|c|c|c|c|c|c|}
\hline \multirow[b]{2}{*}{$\mathrm{CO} 2$} & \multicolumn{2}{|c|}{ MAL $E$ OESTS } & \multicolumn{2}{|c|}{ NIS DEFTH } & \multicolumn{2}{|c|}{ AVEFGGE } \\
\hline & 454 & 158 & 300 & 1465 & 395 & Fon \\
\hline CONS I!! & 627 & 1425 & $\begin{array}{lll}5: 7 \\
0.7\end{array}$ & 1507 & 580 & ppin Hacl \\
\hline COND OUT & 529 & 143 & 350 & 1658 & 871 & ppa $\mathrm{NaCl}$ \\
\hline H2S & 0 & & $\dot{0}$ & & $\dot{\theta}$ & $\mathrm{Fp \#}$ \\
\hline HYOC SRS & 0 & & 0 & & 0 & whits \\
\hline HH IN & 5.9 & tabs & 8.7 & $1.559^{\circ}$ & 8.8 & $15 / 9$ \\
\hline MH OUT & 8.9 & $1680^{\circ}$ & 8.8 & $1477^{\circ}$ & 8.9 & ibig̣ \\
\hline $\mathrm{pH}$ IH & 10.4 & $1467^{\circ}$ & 10.0 & $1700^{\circ}$ & 10.2 & \\
\hline pH QUT & 10.7 & $14: 5$ & 10.2 & $17 ! 5$ & 10.5 & \\
\hline TEMP IN & 116 & 1664 & 102 & $1425^{\prime}$ & $11 !$ & $\operatorname{deg} F$ \\
\hline TEKF OUT & 120 & $1660^{\circ}$ & 100 & $1420^{\circ}$ & 117 & $\operatorname{deg} F$ \\
\hline
\end{tabular}

Conn Gas / Trip bas / Methane : No Hydracartoris
CARBIDE LAG diss ', units, SPH =

Actual Lag $=$ \% Thiecr OPEN HOLE Lag (pres, I punp eff): Nud VIS out $=, 500$ oras Carbide, Kinutes Duration 1

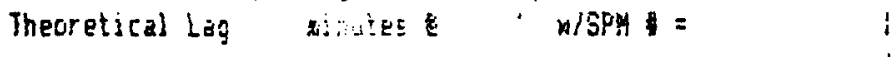
BIT EETRO

\begin{tabular}{|c|c|c|c|c|c|}
\hline & & & CUY & $\mathrm{CH}$ & \\
\hline $0.5 I I$ & MAKETIFE & $5: 118$ & 516 & HS: & CUHDIT:OH \\
\hline 26 & SE: 584 & 148 & $670^{\circ}$ & $\dot{H}$ & 145 \\
\hline
\end{tabular}

\footnotetext{
Report by: BILL GILMous:
}

\section{LITHOLESY}

Genera! Description: \{See DIGITAl. WUO LOG for details\} LITHIC TUFF - YEL WHT ASH H/ AENDT 1-3 HH PERLITE LAPILL!, IHCREASINE W/ DEPTH TO $1580^{\circ}$ WHERE PERIIIIC OBSIDIAN LAFIILI COMPRISE UF TO 952 OF THE TOTAL LITHOLOEY. $1590^{\circ}$ A $10^{\circ}$ YELLOH ORAHGE FHYOLITE FLOH COMPRISES ALL OF THE SAMELE. GELOH THE HELL FRESERVEO GHYOLITE FLON EXISTS A VELLOH-ORAMGE ASH 20HE WITH INCREASIMG PERLITE TO $1650^{\circ}$ HHERE A THICX UWIT OF A YELLOW GREEN DENSE UITAIC TUFF UNIT DOHIKATES $1017000^{\circ}$. THE YELLOH GREEN VITRIC TUFF IS DEHSE, GLASSY, TKAHSLUJEEHT, VITREOUS WITH LESSER CHALKY, ASHY, MICKOVESICLES, RARE EAHDING, POSSIELY COLORED OUE TO CHLORITIC STAIHIHG.
Sanple Shaker Sereen Size: II=40/60 \$2=40/60 MESH

Lotest Serren Size Chang̣e on: 8-2-89 (Start of zell) !

Salple Disposition and Tránsfers:

Dáte:

Interval: Frea to

Sent tolfeceived iy: 


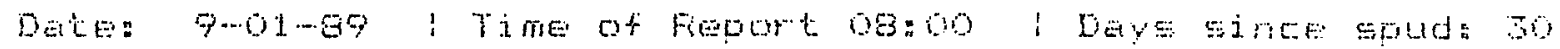

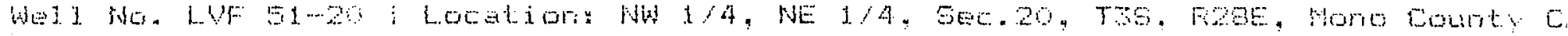

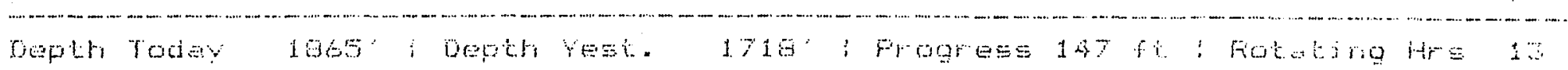

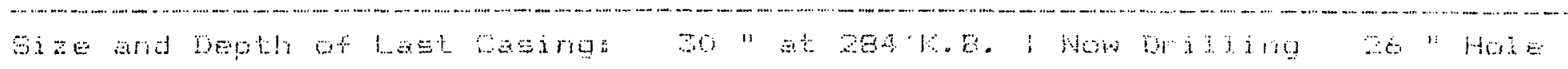

Bit. Doitan

How bet : $\quad$ : ! $\quad$ ! $\vdots \quad \vdots \quad 3$

\begin{tabular}{|c|c|c|c|}
\hline \multirow[t]{6}{*}{ atbol wy } & $\vdots$ & \multicolumn{2}{|c|}{ 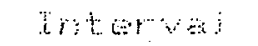 } \\
\hline & $!$ & $170 \ldots$ & 175 \\
\hline & ; & i 20 & Hen \\
\hline & $r$ & $\cdots$ & \\
\hline & ; & $\ldots$ & \\
\hline & $!$ & $\ldots$ & \\
\hline
\end{tabular}

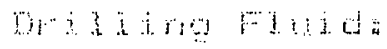

Hed

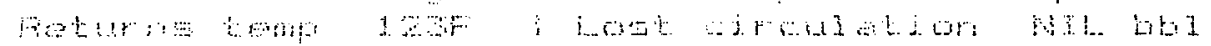

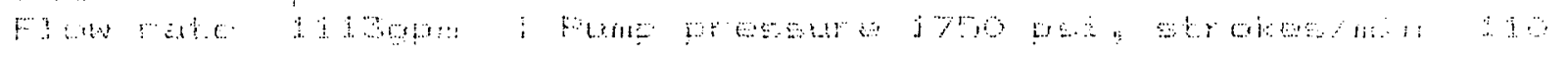

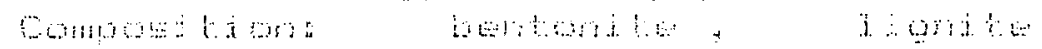

11:0r:

1)

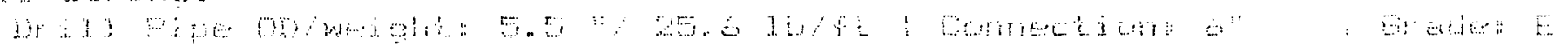

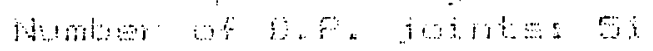

ry

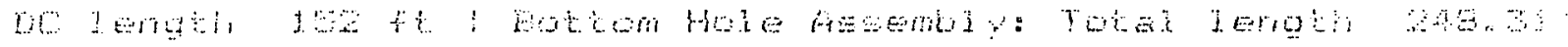

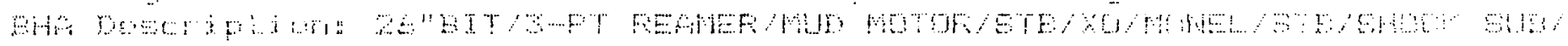

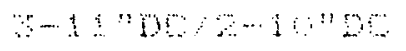

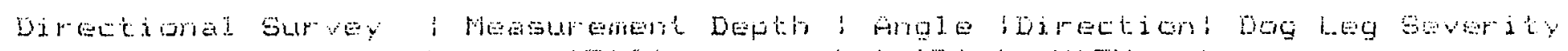

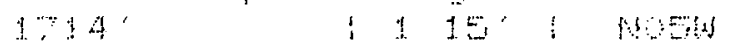

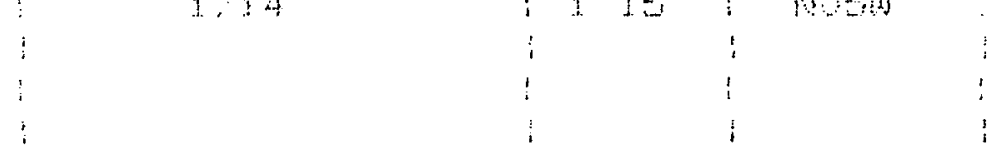

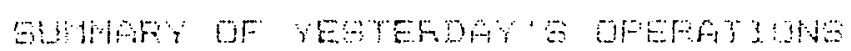

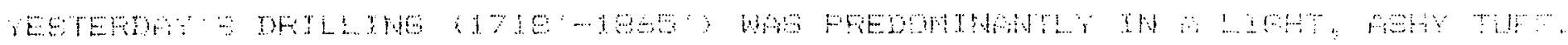

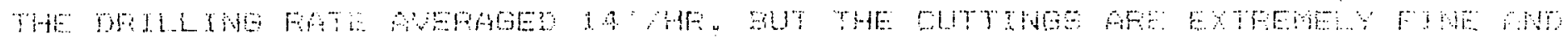

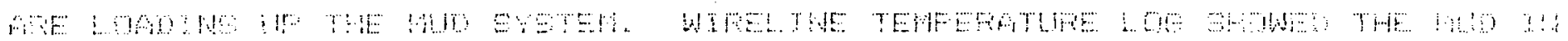

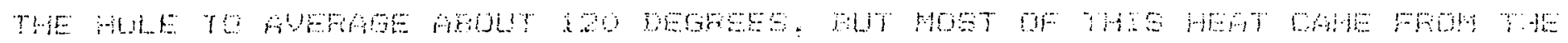

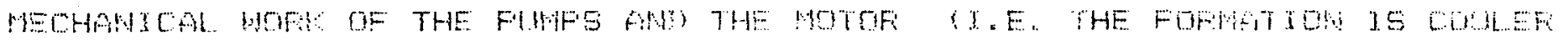

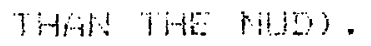

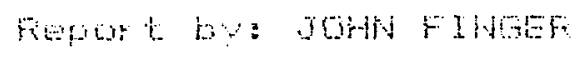


EESEH WE: LDEESHG

DOE/SANDIA MAGMA ENEREY WELL LUF 51-20 DAILY MUD LQE FEFUFT

\begin{tabular}{|c|c|c|}
\hline Reptet Dete & $6-6 !-85$ & 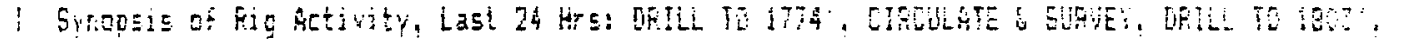 \\
\hline Feport Tibe & $68: 50$ & 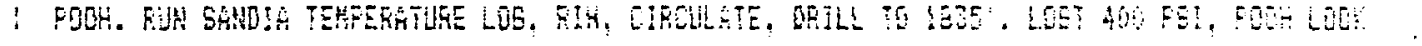 \\
\hline Do Eince Spud & 5 & 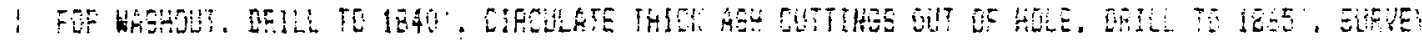 \\
\hline tre $\equiv Q:$ t lestiti & IE: & $\vdots$ \\
\hline Fride Deftis & $17:$ & : \\
\hline 24 ir sirtacte & 177 & 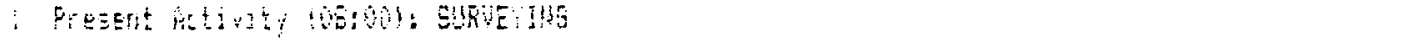 \\
\hline Heditg dis & 13 & $\vdots$ \\
\hline FUE THF $\mathrm{y}$ SIKE & $i$ & 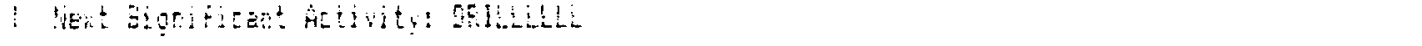 \\
\hline Fif is in Foetit & How & $\vdots$ \\
\hline
\end{tabular}

\begin{tabular}{|c|c|c|c|c|c|c|c|c|}
\hline \multirow[b]{3}{*}{ Eot } & \multicolumn{6}{|c|}{ 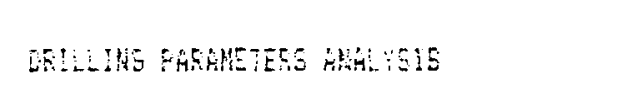 } & \multicolumn{2}{|r|}{ WTyn: } \\
\hline & $5 \%$ & EDFi: & HOE & DEPTH & AUE: & HEE & $\vdots$ & 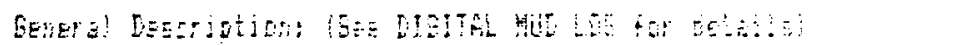 \\
\hline & 42 & 1999 & is & 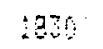 & 14 & D. FGTE & ! & 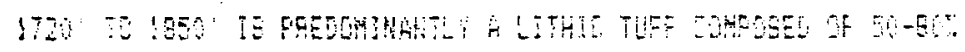 \\
\hline Hes & $1 !$ & $\$ 743^{\circ}$ & $\Xi$ & 1794 & $\dot{0}$ & $1-125$ & ! & 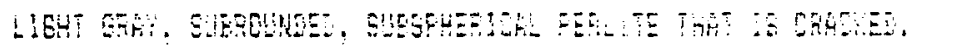 \\
\hline GE: & $3 t$ & 1774 & 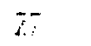 & 1725 & 3 & & $i$ & 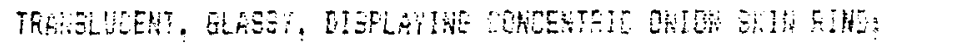 \\
\hline TFî & 14 & 37 & $\bar{i}$ & 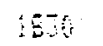 & & d畔引三 & $!$ & 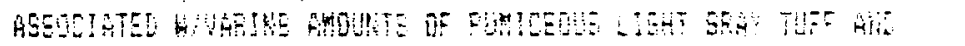 \\
\hline 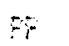 & 203 & 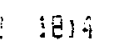 & 154 & $1: 2$ & 1510 & $\mathrm{PSi}$ & $\vdots$ & 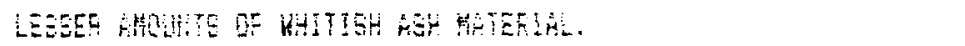 \\
\hline
\end{tabular}

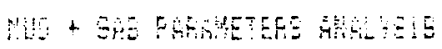

\begin{tabular}{|c|c|c|c|c|c|c|}
\hline & 40 & nE? & mid & DEFIO & HIE & $E$ \\
\hline$\therefore$ & 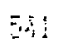 & 51 & $3:$ & 48 & +12 & 5 \\
\hline BHE IS & $2 \mathrm{~s}$ & $4 E$ & $5:$ & 1848 & 598 & pots: 1180 \\
\hline Wia & 038 & 1817 & 798 & 179 & 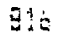 & get hes \\
\hline $\mathrm{HiE}$ & $\dot{s}$ & & 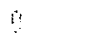 & & $\therefore$ & $p 5$ \\
\hline 90062 & $i$ & & ij & & 0 & whits \\
\hline 48 & $E .5$ & :sen & E. & 172 & $\Xi 5$ & itis \\
\hline sis & 5. & .908 & $\bar{E} .5$ & 1798 & 5.5 & its \\
\hline ED & 19.5 & $12 \mathrm{t}^{\prime}$ & 9.5 & 175 & 1.1 & \\
\hline of out & 10.3 & $1809^{\circ}$ & 16.2 & 1722 & 10.3 & \\
\hline TEE YK & $\because Y$ & 979 & 112 & 190 & 198 & $\dot{d e g} 5$ \\
\hline 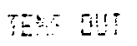 & $2:$ & 1750 & $! !-$ & 1514 & 12 & SEL \\
\hline
\end{tabular}

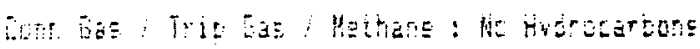

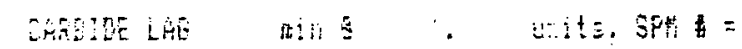

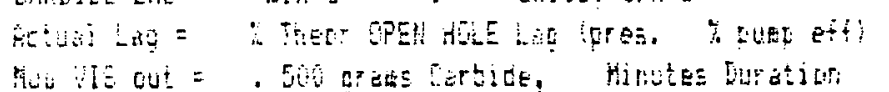

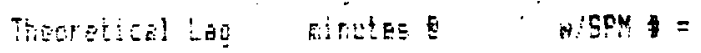

SUT EESTE

\begin{tabular}{|c|c|c|c|c|c|}
\hline & & & Cuti & 54 & \\
\hline M. EUIE & HOE T:PE & It: & TTE & HES & Bolog:Tak \\
\hline $5 \quad 20^{\prime}$ & $S E I B E$ & 148 & $5: 7$ & 45 & INS \\
\hline
\end{tabular}

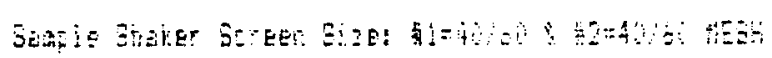

Latest Screen Size thange on: 8-2-8? istant of well:

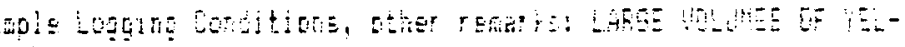

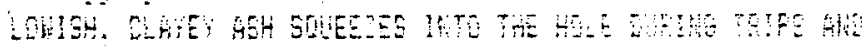

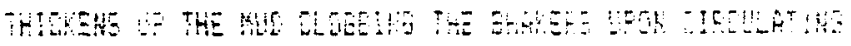
buTtors UF.

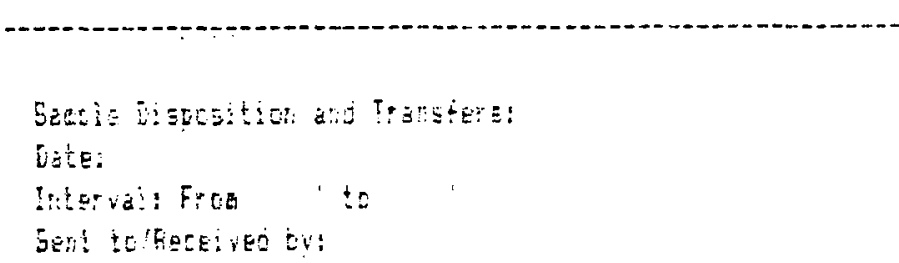

Reurer: tr: EILL EILNGSE 


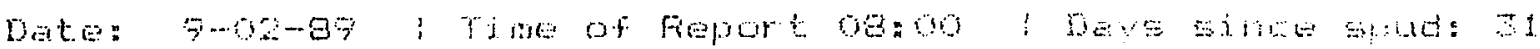

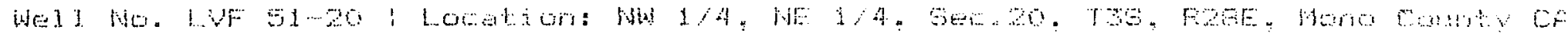

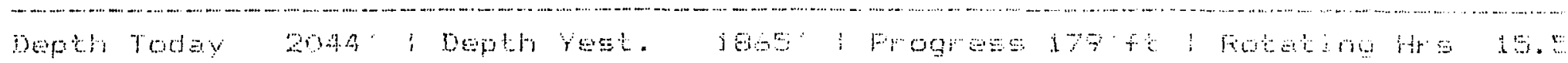

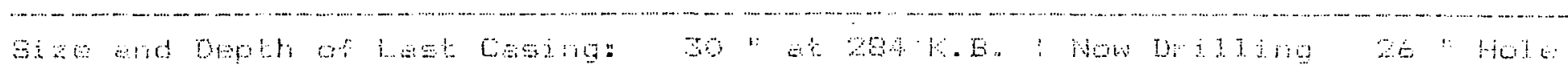
Bitiotion

5.

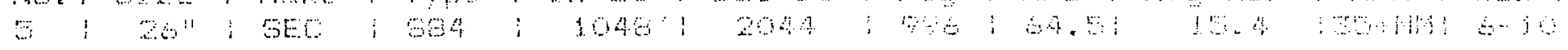

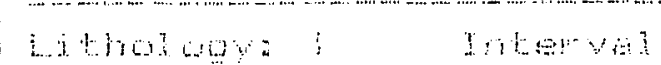
josto anol खो 2040 अ 45

\section{!}




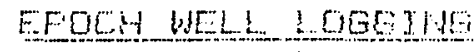

DOE/SANDIA MAGMA ENEREY WELL LVF 51-20

DAILY MUD LOG FEFOFT

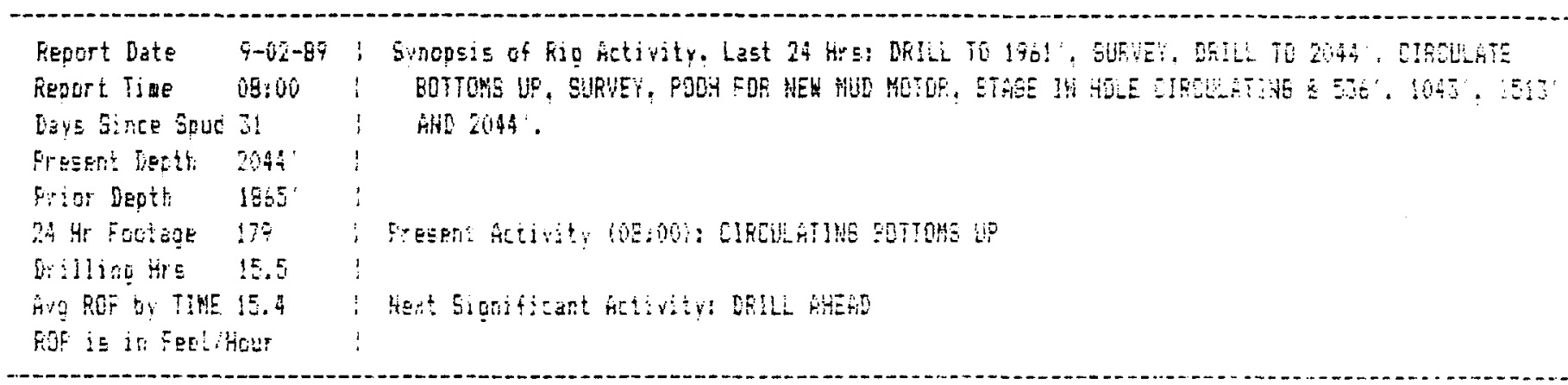

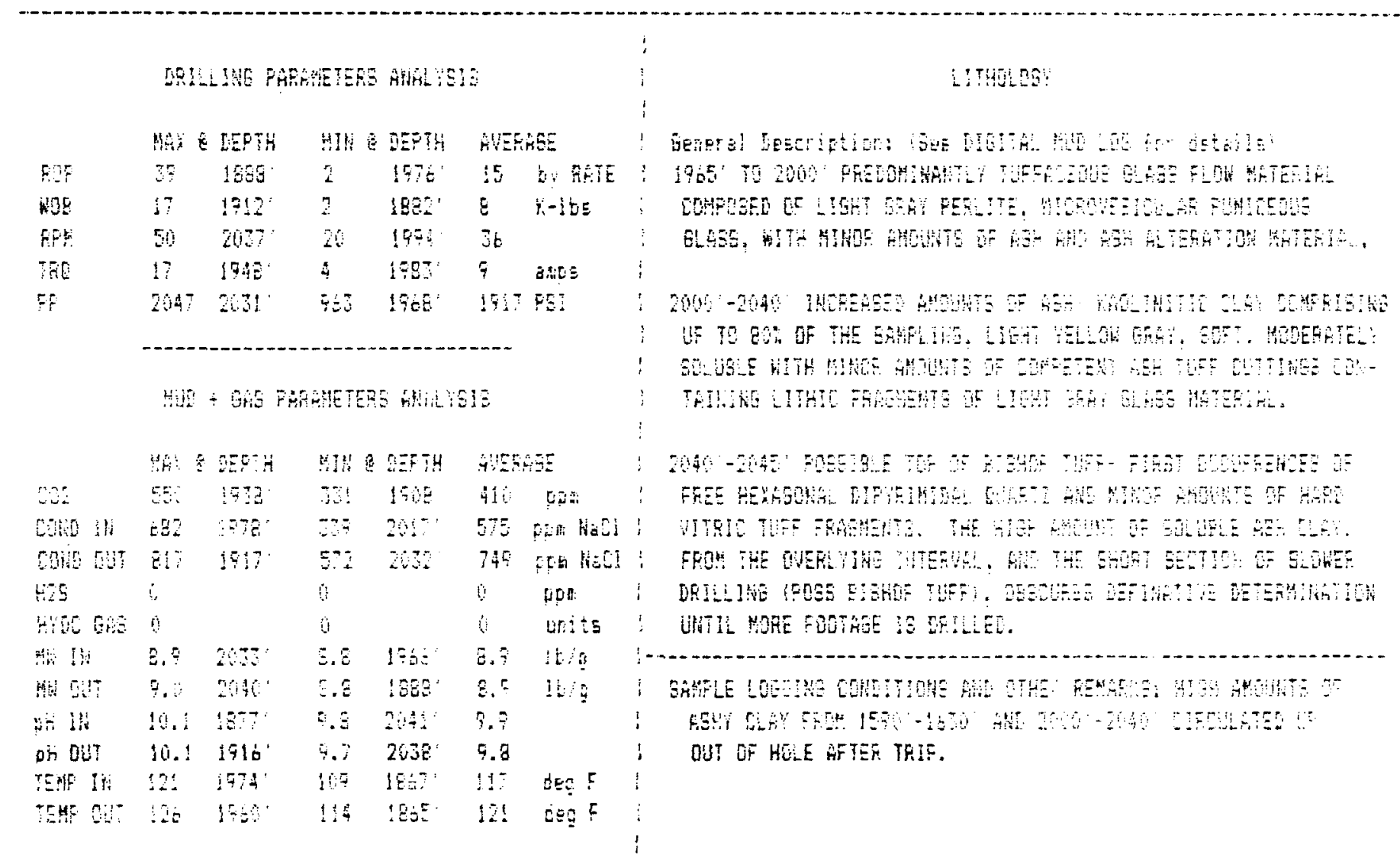

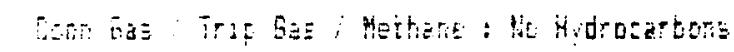

Coretite bis bis

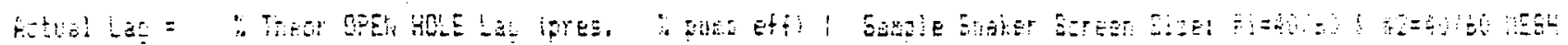

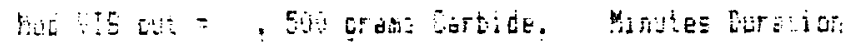

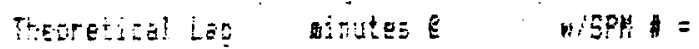

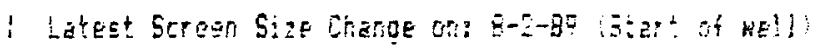

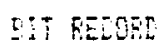

\begin{tabular}{|c|c|c|c|c|c|}
\hline & & & $\therefore$ & rits & \\
\hline HD. ETRE & MAEETTE & $1: 9$ & 79 & 485 & conitsots \\
\hline $5 \quad 20^{5}$ & SEE 9E4 & 1040 & 94 & 64.5 & 6]5 \\
\hline
\end{tabular}

Report i: bll! EIIMOUE 


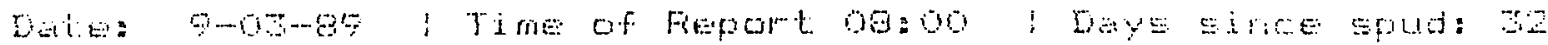

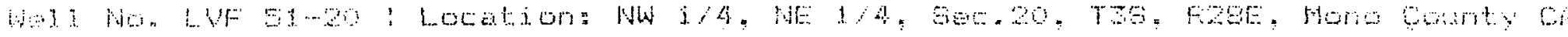

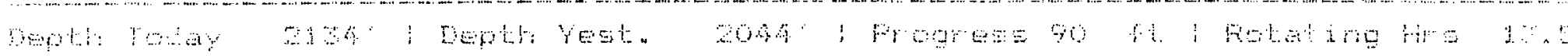

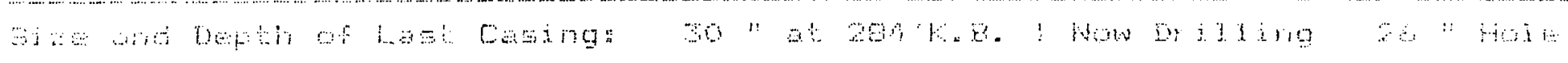

Yat

Hon ! +

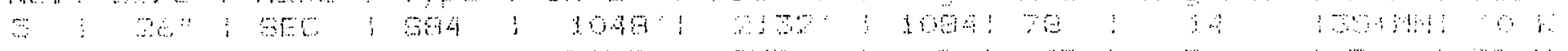

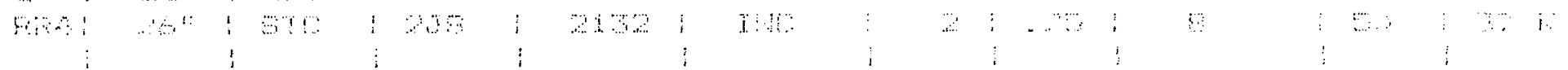

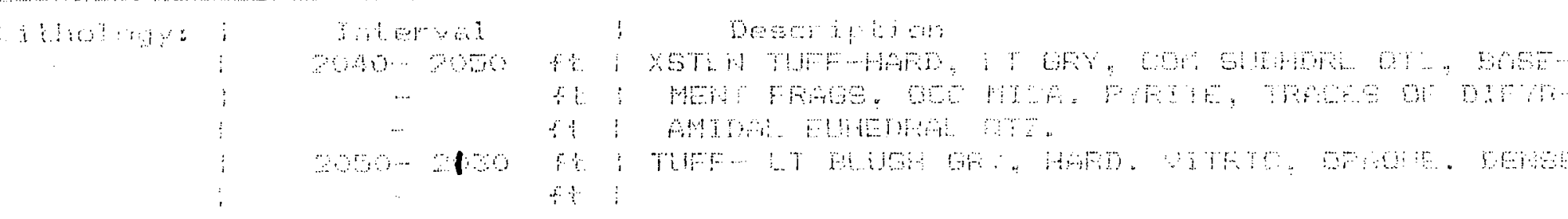

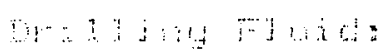

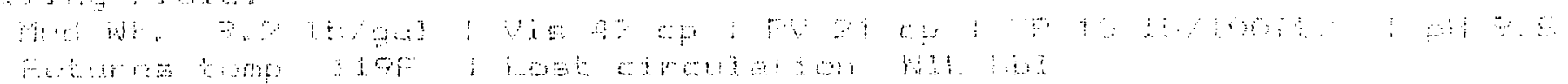

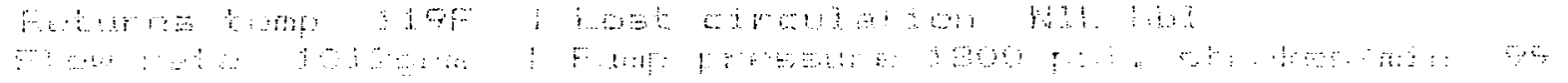

\%...

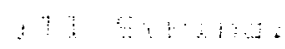

\&

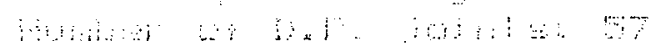

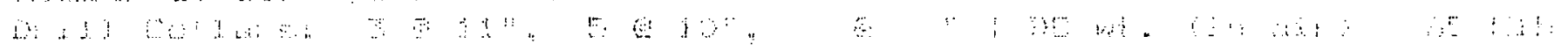

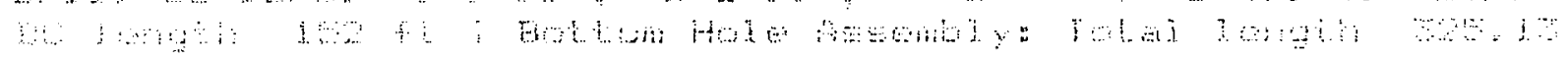

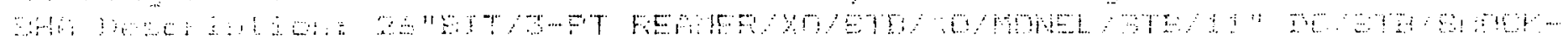
का:

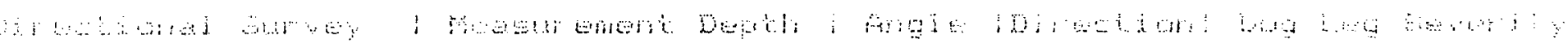

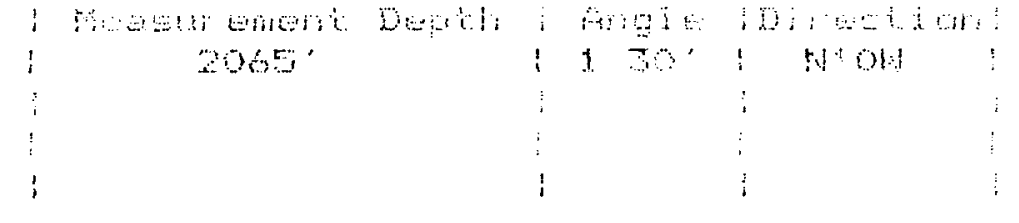

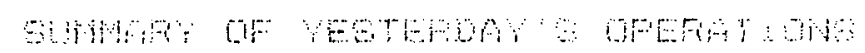

मа

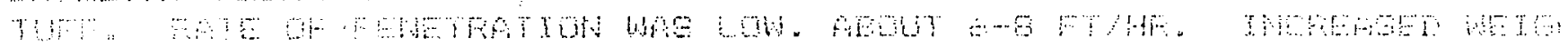
ac :

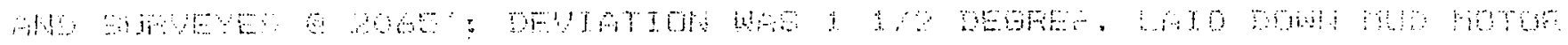

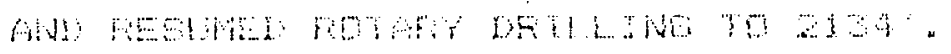

$\therefore$ a. 


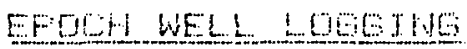

DOE/SANDIA MAGMA ENERGY WELL LUF 51-20 DAILY MUD LOG FEFURT

\begin{tabular}{|c|c|c|}
\hline Report Date & $9-03-89$ & 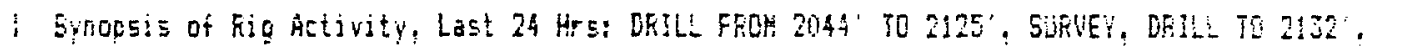 \\
\hline Regorti Tige & $08: 00$ & 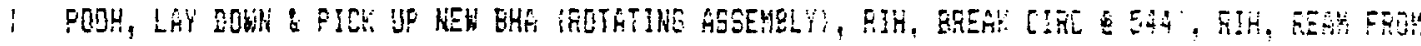 \\
\hline Deys Since Etud & 32 & 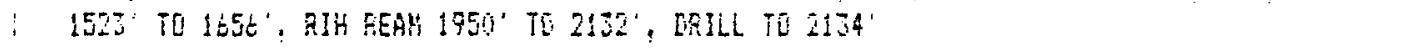 \\
\hline Frior Depth & $2044^{\circ}$ & i \\
\hline 24 4: Foutạte & 90 & 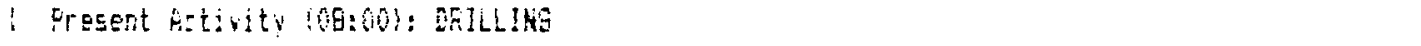 \\
\hline 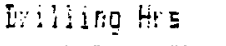 & 13.5 & $\vdots$ \\
\hline COF iE II FEEL $t$ & & $!$ \\
\hline
\end{tabular}

\section{DRILLINE FGEAMETERE AUALISES}

\begin{tabular}{|c|c|c|c|c|c|c|}
\hline & 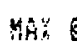 & DEPTH & 1:48 & MEFTS & \multicolumn{2}{|c|}{ HICKAEE } \\
\hline rof & 23 & 205 & 2 & $700 \mathrm{j}$ & 3 & DV FAOE \\
\hline DiE & 25 & $2167^{\circ}$ & $E$ & $2040^{\circ}$ & 14 & $x-3 x$ \\
\hline Fet: & 54 & $2110^{\circ}$ & 25 & $2114^{\circ}$ & $3 i$ & \\
\hline$T E[$ & 3 & $2094^{\circ}$ & $b$ & 2124 & $! \vdots$ & 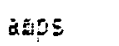 \\
\hline$F F$ & 207 & $2106^{\prime}$ & 1000 & $2114^{\circ}$ & 185 & FSI \\
\hline
\end{tabular}

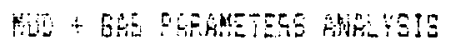

\begin{tabular}{|c|c|c|c|c|c|c|}
\hline & Pit: & DEF & M:1 & DEFTH & \multicolumn{2}{|c|}{ PUESAE } \\
\hline 02 & 象5: & $2650^{\circ}$ & 3 & 204 & $37 !$ & pog \\
\hline Corw in & 075 & 2008 & 418 & $267^{\circ}$ & Stot & EqE HES \\
\hline Coltr git & 625 & 205 & 572 & $2032^{\circ}$ & $01=$ & pota HaC \\
\hline$H 25$ & 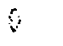 & & 0 & & 8 & تED \\
\hline HOE EHE & ? & & 8 & & 1 & unit:s \\
\hline$\mu_{3}: 31:$ & $i . i$ & 207 & $E .5$ & 205 & 7.0 & ltin \\
\hline Mont & ร. & $204=$ & 9.6 & 2122 & 5.0 & $35: 8$ \\
\hline ph If & $9 . \mathrm{E}$ & 2043 & 9.0 & 2132 & 9.7 & \\
\hline DH QU? & 9.7 & 2045 & 9.6 & $2129^{\circ}$ & 9.6 & \\
\hline TEKE $3 K$ & 25 & 2122 & 114 & $2047^{\prime}$ & 120 & Gso $=$ \\
\hline TEF: QST & 122 & $\because 2 \mathrm{O}$ & $1: 5$ & 2045 & 125 & $d e f$ \\
\hline
\end{tabular}

\section{LIHEL:}

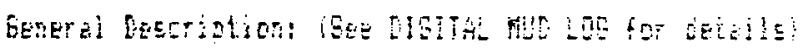

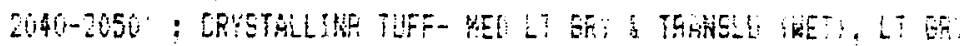

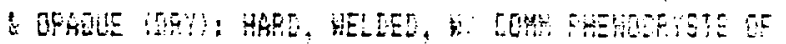

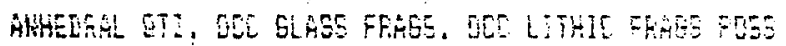

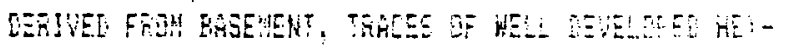

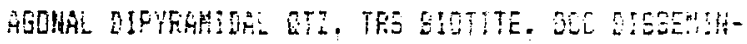
ATED PUITE. - ESHAT THEF-

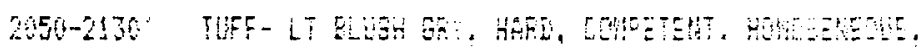

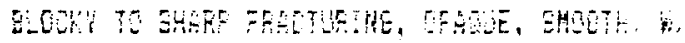

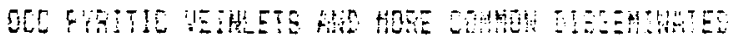

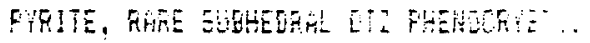

Cont: bas Trif Ses/ Rettiane : kic Hudrotzrbone

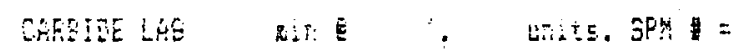

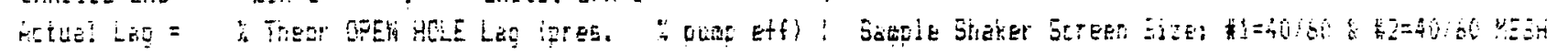

Huc $v 1 S$ out $=$, 500 grans Carbide; Minutes buration :

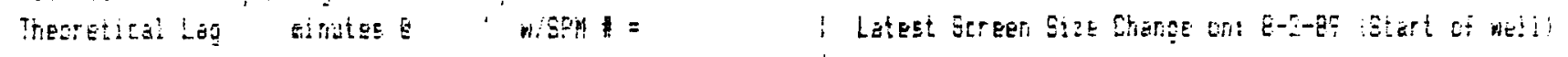
-

\section{DiT SECHRD}

\begin{tabular}{|c|c|c|c|c|c|}
\hline & & & $\mathrm{CH}$ & W's & \\
\hline HO. SIZ & MHETTEE & $2 N 6$ & FIE & HES & agh: \\
\hline $5 \quad 3$ & SE: SES & $104 \hat{0}$ & $\operatorname{lig} 5$ & 79 & $600 \mathrm{E}$ \\
\hline
\end{tabular}

Beport by: E!L! GiLHOLS

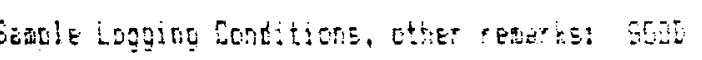


EPOCH WELL LOGEIME

DOE/SANDIA MAGMA ENERGY WELL LUF 51-20

DAILY MUD LOG REPORT

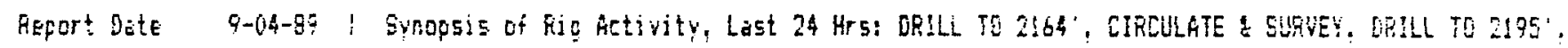

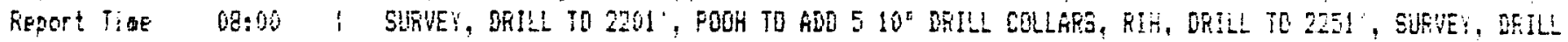

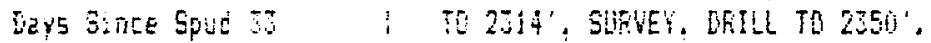

Freserit Detth 2350

Friar Dejth $2 ! 34$

24 ir Fortage 216

Erilling itrs lis.

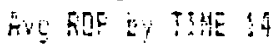

For is in Fetihut

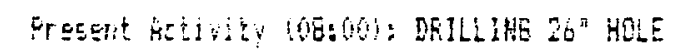

Hex Eignificant Hetiwity: Dorth

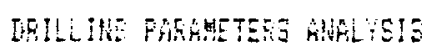

\begin{tabular}{|c|c|c|c|c|c|c|}
\hline & 48 & SEFTH & $4 i$ in & BEFTH & RUERH & \\
\hline WF & $7 !$ & $21 \leq 2$ & 4 & 290 & 17 & DV SRTE \\
\hline WE & 50 & 2247 & 18 & $2 ! 61$ & 35 & $1-15.5$ \\
\hline he & $T E$ & 2242 & 50 & 2154 & $b !$ & \\
\hline $7 \mathrm{RS}$ & 3 & 282 & 7 & 2135 & 21 & s \\
\hline$\%$ & $210 !$ & $2 \mathrm{ZlE}$ & 837 & 2151 & 185 & 5 \\
\hline
\end{tabular}

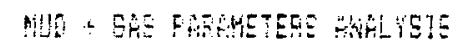

\begin{tabular}{|c|c|c|c|c|c|c|}
\hline & 184 & DEFTH & sil: & BEFHH & GHEF & \\
\hline$\because$ & 497 & 230 & 349 & 298 & $4: 7$ & tizi \\
\hline Efiri 13 & 070 & 245 & 410 & 220 & EIE & yos $k_{a}$ \\
\hline 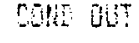 & $\vdots 15$ & 215 & Eje & 298 & 212 & 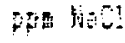 \\
\hline 15 & 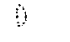 & & $\because$ & & 4 & pot: \\
\hline 0.695 & : & & ! & & 8 & writs \\
\hline 14 & 9.0 & $2 \mathrm{rol}$ & 9.7 & 7206 & 9.0 & ibio \\
\hline 1051 & 9.1 & 218 & 5.7 & 231 & 5.6 & b. \\
\hline 3 & 5 & $290^{\circ}$ & 5.7 & 250 & 7.5 & \\
\hline $\mathrm{eur}$ & 9.8 & 2300 & 9.6 & 2156 & 9.7 & \\
\hline EFF II & 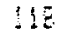 & 20 & 165 & 206 & 114 & $\therefore e c F$ \\
\hline HE OUT & 123 & 2156 & :1i & $215 \bar{\varepsilon}^{\circ}$ & 117 & $\operatorname{tin}{ }_{3} F$ \\
\hline
\end{tabular}

1154089

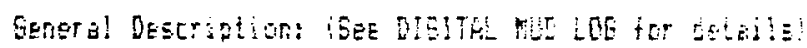

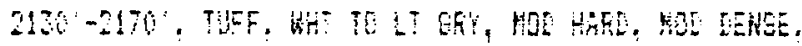

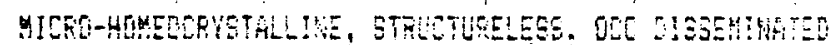

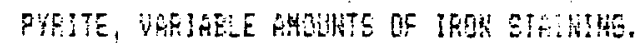

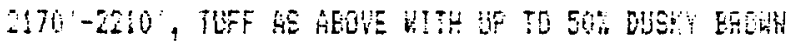

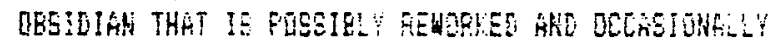

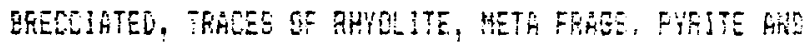

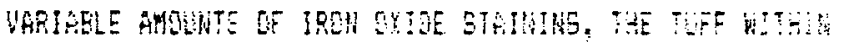

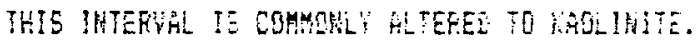

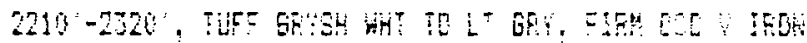

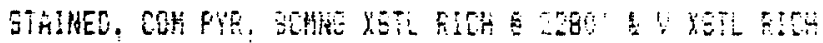
$140 ; 2001$.

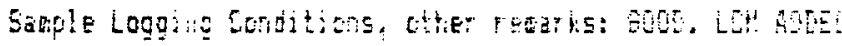

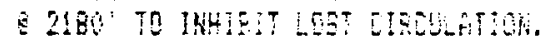

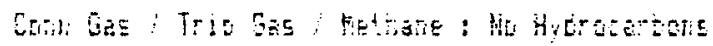

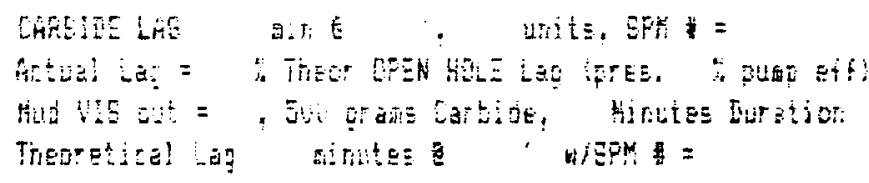

Bit Rersis

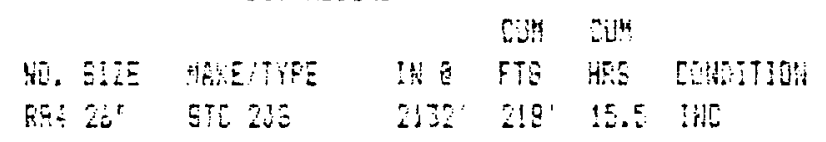

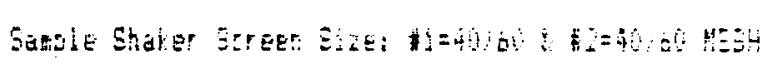

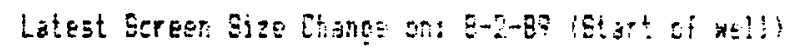
$-1$ 


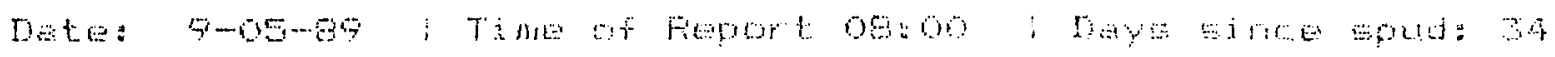

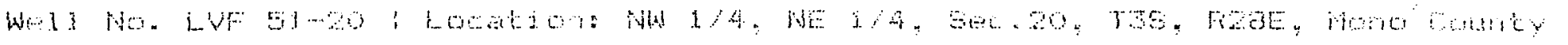

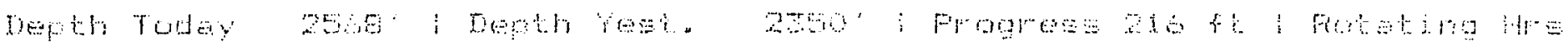

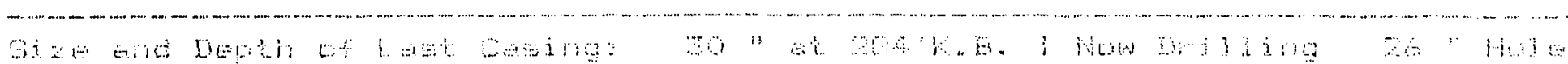
e.t. batas:

16.

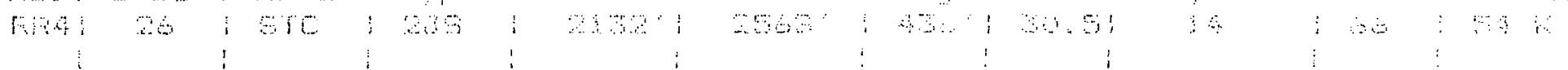
:

$\sin \cos \theta \sin$

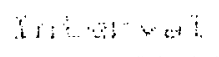
$\because \cdots, \cdots$ $10-$ $\operatorname{mon}+\sin$

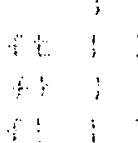

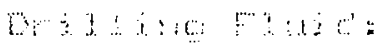
Hed iti.

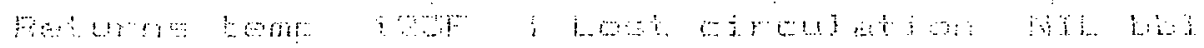

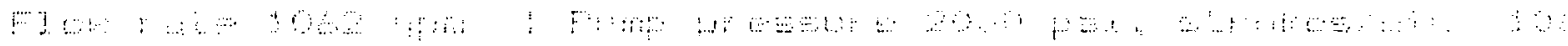

Compers : in:

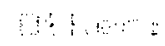

i: a: $:$ :

7.

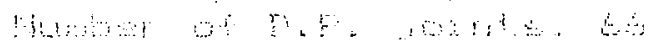

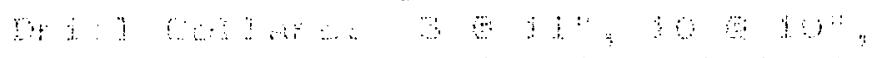

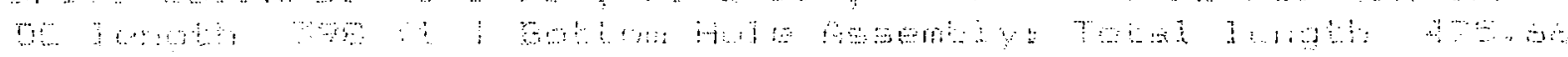

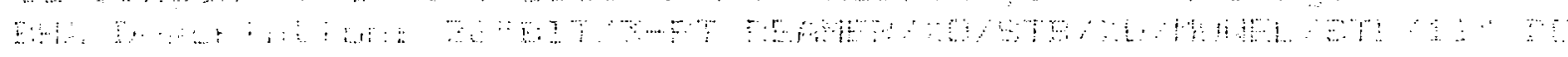

का

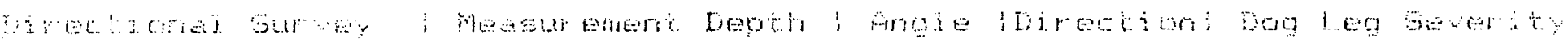

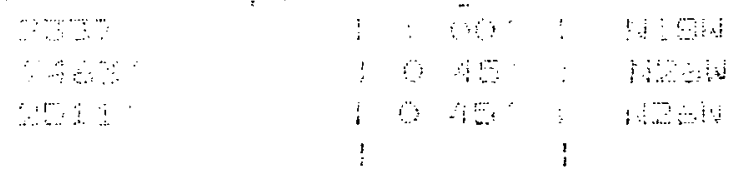

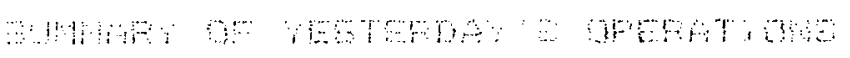

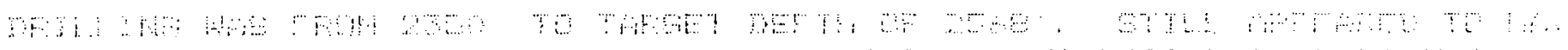

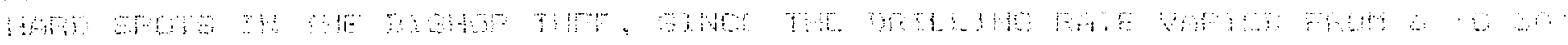

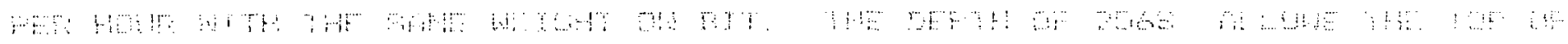
औH

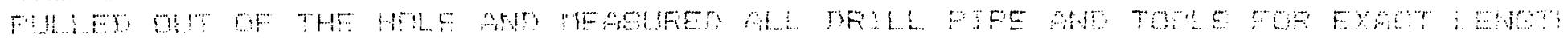

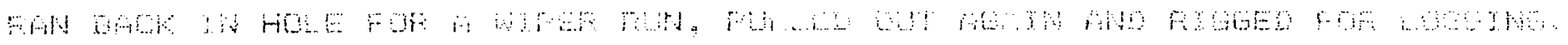

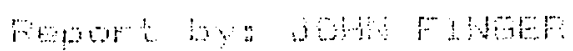




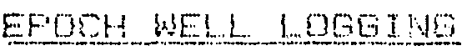

\section{DOE/SANDIA MAGMA ENERGY WELL LUF $51-20$ DAILY MUD LOG FEFOFT}

\begin{tabular}{|c|c|c|c|}
\hline Eeport Date & $G-05-E ?$ & $\vdots$ & 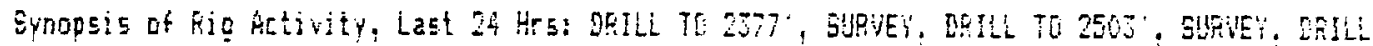 \\
\hline Regort line & $08: 00$ & $\vdots$ & 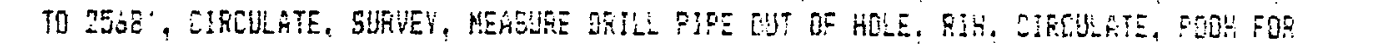 \\
\hline Löte Sinte Eput & 34 & $i$ & E-LBES. \\
\hline Fresent Defth & $2568^{\prime}$ & $\vdots$ & \\
\hline Friol bett: & 2350 & $\vdots$ & $\therefore$ \\
\hline if Hr Fortiege & 218 & i & 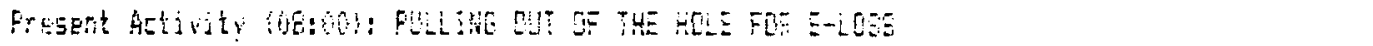 \\
\hline 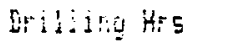 & 15 & $\vdots$ & \\
\hline $\begin{array}{l}\text { Hy Fe by Tide } \\
\text { HOF is in FeEt }\end{array}$ & & $\vdots$ & 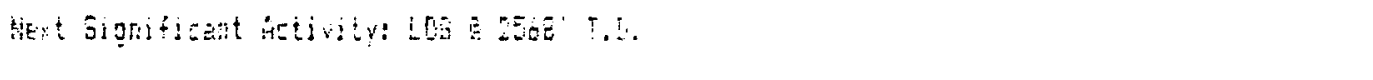 \\
\hline
\end{tabular}

\begin{tabular}{|c|c|c|c|c|c|}
\hline \multicolumn{6}{|c|}{ TRILLUO GARAETESE MHALSIS } \\
\hline & HAB: & A UEFTH & $M T H E$ & EEPTH & AVERABE \\
\hline nif & TE & 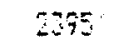 & $\Xi$ & 2559 & It ty Grít \\
\hline HeS & 5 & $25,3^{\circ}$ & $: 2$ & 2437 & Jo $\quad 1-15=$ \\
\hline 502 & 74 & 2595 & 35 & $242 E^{\prime}$ & s! \\
\hline Thi & 30 & 20 & 13 & 2472 & He \\
\hline 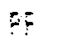 & $2: 35$ & 2,40 & 1075 & 250 & 30450 \\
\hline
\end{tabular}

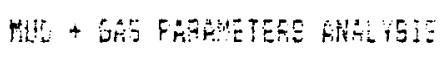

\begin{tabular}{|c|c|c|c|c|c|c|}
\hline & 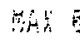 & DEF & 勧 & DEFTH & HEE & AGE \\
\hline $5 \%$ & $\therefore 2$ & 243 & 342 & $2=01$ & 414 & $=2 x$ \\
\hline Dots In & 58 & $2=0$ & $3 !$ & 2532 & 425 & 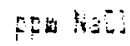 \\
\hline mage & :Ut & 251 & $=\mathrm{Bi}$ & 2549 & 62 & Foh Hall? \\
\hline HS & $\because$ & & ! & & 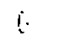 & tot: \\
\hline 4253 & $\because$ & & 8 & & 6 & mits \\
\hline mit & $\because .1$ & $75: 4$ & 5.7 & 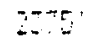 & 5.0 & 19 \\
\hline 40 & 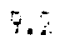 & $25 e$ & 6.0 & $\therefore=5$ & $\vdots .1$ & it: \\
\hline (x) lit & 7.7 & $24: 1^{\circ}$ & 9. & $25+7$ & 3.5 & \\
\hline PH OWT & 9.7 & 239 & 9.6 & $2530^{\circ}$ & $5 . \dot{b}$ & \\
\hline TEKE IK & $\$ 19$ & $2490^{\circ}$ & $1 ! !$ & $23:{ }^{m}$ & 115 & seg: \\
\hline TEHE SUS. & 24.7 & $255 !$ & $11:$ & $24 ! 0^{\circ}$ & $2 z$ & dec $F$ \\
\hline
\end{tabular}

\section{STHE:}

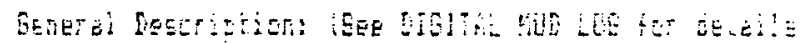

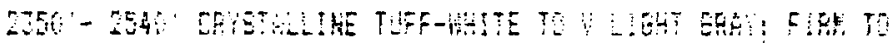

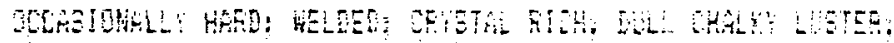

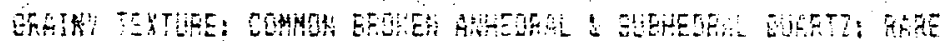

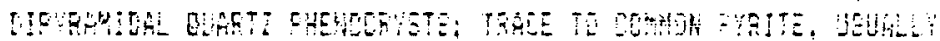

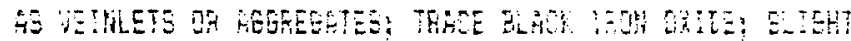

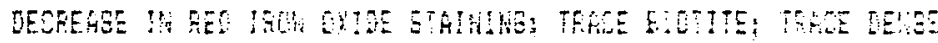

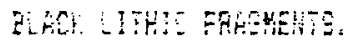

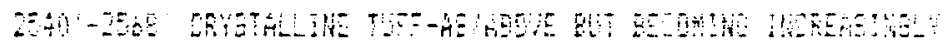

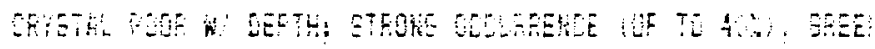

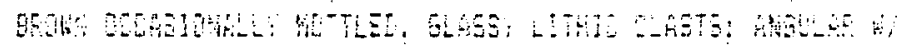

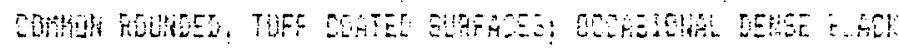

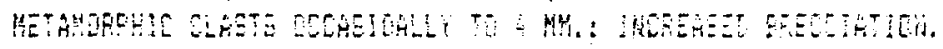

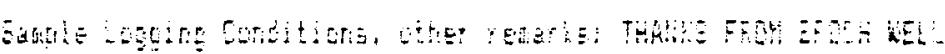
86:5!:

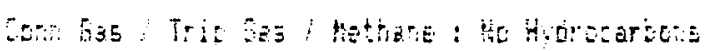

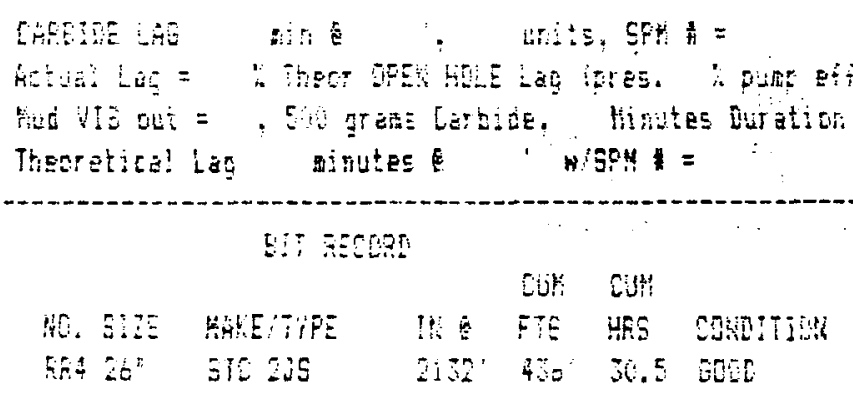

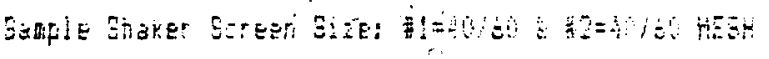
Letest Gcreer Size Change on: 8-2-39 istart of no?? 


\section{DISTRIBUTION}

David N. Anderson

Geothermal Resources Council

P. O. Box 1350

Davis, CA 95617

Roger Anderson

Lamont-Doherty Geological

Observatory

Borehole Research Group

Palisades, NY 10964

John Arestad

Santa Fe Geothermal

2 Galleria Tower

13455 Noe1 Rd., Suite 1100

Dallas, TX 75240

Don Ash

5376 Sharon Ct.

Santa Rosa, CA 95405

Roy A. Bailey

Geological Survey

345 Middlefield Rd., MS-910

Menlo Park, CA 94025

Bureau of Land Management (3)

Attn: Bob Kohlbush

Pat Gallagher

Hal Stoops

300 Federal Building

800 Truxton Ave

Bakersfield, CA 93301

Jay Cass

California Regional Water

Quality Control Board

15371 Bonanza Rd.

Victorville, CA 92392

Cheryl Closson

California Energy Commission

Development Division

1516-9th St., MS-43

Sacramento, CA 95814

Neal Davis

Chevron Service Co.

Drilling Technology Center

P. O. Box 4450

Houston, TX 77073
Robert Deputy

ARCO Oil \& Gas Co.

2300 W. Plano Parkway

Plano, TX 75075

Epoch (3)

Attn: Bill Gilmour

Doug Milham Mike Campbel1

1884 Eastman Ave., \#104

Ventura, CA 93003

Michael Ferguson

Bureau of Land Management

787 N. Main, Suite $P$

Bishop, CA 93514

Charles George

Halliburton Services

Drawer 1431

Duncan, OK 73536-0408

Barry Harding

Ocean Drilling Program

Texas A\&M University

1000 Discovery Dr.

College Station, CA 77840

Charlena Harrel

Okie-Yoke

P. O. Box 105

Lindsay, OK 73052

John F. Hermance

Brown University

Dept. of Geol. Sciences

Providence, RI 02912

David P. Hill

U. S. Geological Survey

345 Middlefield Road, MS-977

Menlo Park, CA 94025

Stephen Howard

Ocean Drilling Program

Texas A\&M University

1000 Discovery Dr.

College Station, CA 77840

A. P. S. (Tony) Howells

Atlas Wireline Services

P. O. Box 1407

Houston, TX 77251 
Wayne Jackson

Jackson Equipment Co.

P. 0. Box 669

Middletown, CA 95461

Mike Jarvis

Mammoth Times

P. 0. Box 3929

Mammoth Lakes, CA 93546

James W. Langford

Security Division

Dresser Industries, Inc.

P. 0. Box 210600

Dallas, TX 75211-0600

Tim Linscott

Santa Fe Geothermal

2 GAlleria Tower

Suite 1100

13455 Noel Rd.

Dallas, TX $75240=-6620$

B. J. Livesay

1525 Elon Lane

Encinitas, CA 92024

Loffland (3)

Attn: Gene Anderson

Vern Miller

Duey Milner

P. 0. Box 418

Bakersfield, CA 93302

William Lyons

New Mexico Institute of

Mining \& Technology

Socorro, NM 87801

Danie1 L. Lyster

Mono County Energy Mgmt.

P. 0. Box 8060

Mammoth Lakes, CA 93546

M-I Drilling Fluids (4)

Attn: Sid Johnson

George Grundt

Larry Stiner

Boyd Green

3160 Telegraph Rd., Suite 207

Ventura, CA 93003
Doc McMillan

Halliburton

10816 Shellabarger Rd.

Bakersfield, CA 93312

Nic Nickels

Eastman Christensen

3636 Airway Drive

Santa Rosa, CA 95403

J. J. Papike

South Dakota School of Mines and Technology

501 E. St. Joseph Street

Rapid City, SD 57701-3995

Steve Pye

Unocal Geothermal

P. 0. Box 7600

Los Angeles, CA 90017

Ted Reeves

Chaffey High School

1245 N. Euclid Ave.

Ontario, CA 91762

Bill Rintoul

2721 Beech St.

Bakersfield, CA 93301

John Rundle

Earth Science Dept.

L-201, LLNL

Livermore, CA 94550

John H. Sass

Geological Survey

2255 North Gemini Drive

Flagstaff; AZ 86001

L. T. Silver

Division of Geological and

Planetary Sciences

California Institute of Technology

Pasadena, CA 91125

Michael Smith

Geothermal Program Manager

California Energy Commission

Development Division

1516-9th St., MS -43

Sacramento, CA 95814 
Michael L. Sorey

Geological Survey

345 Middlefield Rd, MS-439

Menlo Park, CA 94025

Michael A. Storms

Ocean Drilling Program

Texas A\&M University

College Station, TX 77843-3469

Gene Suemnicht

Unocal

3576 Unocal Place

Santa Rosa, CA 95406

William T. Taylor

The Town of Mammoth Lakes

P. 0. Box 1609

Mammoth Lakes, CA 83546

Robert D. Tibbs

CE Exploration Co.

111 SW Fifth Ave., Ste 2150

Portland, OR 97204

Tonto Drilling Services (2)

Attn: George McLaren

Larry Pisto

P. 0. Box 25128

Salt Lake City, UT 84125-0128

U. S. Department of Energy (2)

Geothermal Technologies Div.

Attn: Ted Mock

Gladys Hooper

Forrestal Bldg., CE-324

1000 Independence Ave., SW

Washington, DC 20585

U. S. Department of Energy

Office of Basic Energy Sciences

Attn: George A. Kolstad

Mail Stop J-309

Washington, DC 20585

U. S. Forest Service (3)

Attn: Tom Heller

Dean McAlister

Molly McCartney

Mammoth Lakes, CA 93546
Herbert $F$. Wang

University of Wisconsin

Lewis G. Weeks Hall

1215 W. Dayton St.

Madison, Wisconsin 53706

Dick Yarter

Northern CA Power Agency

P. 0. Box 425

Healdsburg, CA 95448

Tommy Warren

Amoco Production Center

P. 0. Box 3385

Tulsa, OK 74102

3141

$3141-1$

S. A. Landenberger

(5)

C. L. Ward

For DOE/OSTI (8)

3151

W. I. Klein (3)

6000

D. L. Hartley

6200

V. L. Dugan

6233

J. C. Eichelberger

6233

V. S. McConnel

6250

6252

P. J. Hommert

J. C. Dunn

6252

6252

6252

6252

6252

6252

6252

6253

J. T. Finger (20)

R. D. Jacobson (10)

G. E. Loeppke

D. A. Glowka

P. C. Lysne

J. Gabaldon

R. P. Wemple

A. R. Sattler

J. R. Wackerly 